\title{
Surface and Bottom Boundary Layer Dynamics $/ / 4 / 7$ on a Shallow Submarine Bank: Southern Flank of Georges Bank
}

August 1999

(C) Sandra Regina Werner, 1999

The author hereby grants to MIT and to WHOI permission to reproduce and to distribute copies of this thesis document in whole or in part.

Signature of Author -

Joint Program in Physical Oceanography

Massachusetts Institute of Technology

Woods Hole Oceanographic Institution

August 11, 1999

Certified by $\ldots$

Robert C. Beardsley

Senior Scientist

Thesis Supervisor

Accepted by

W. Brechner Owens

Chair, Joint Committee for Physical Oceanography 


\title{
Surface and Bottom Boundary Layer Dynamics on a Shallow Submarine Bank: Southern Flank of Georges Bank
}

\author{
by \\ Sandra Regina Werner \\ Submitted in partial fulfillment of the requirements for the degree of \\ Doctor of Philosophy at the Massachusetts Institute of Technology \\ and the Woods Hole Oceanographic Institution
}

August 12, 1999

\begin{abstract}
The thesis investigates the circulation at a 76-m deep study site on the southern flank of Georges Bank, a shallow submarine bank located between the deeper Gulf of Maine and the continental slope. Emphasis is placed on the vertical structure of the bottom boundary layer driven by the semidiurnal tides and the flow field's response to wind forcing.

The observational analysis presented here is based on a combination of moored array and bottom tripod-mounted current, temperature, conductivity, and meteorological data taken between February and August 1995. Results from the bottom boundary layer analysis are compared to numerical model predictions for tidal flow over rough bottom topography. The flow response to wind forcing is examined and brought into context with the existing understanding of the wind-induced circulation in the Georges Bank region. Particular attention is given to the vertical distribution of the wind-driven currents, whose variation with background stratification is discussed and compared to observations from open ocean studies.
\end{abstract}




\section{Acknowledgments}

First of all, I would like to thank my advisor, Robert Beardsley, for his patience and support over the past 5 and a half years, for his encouragement and advice throughout this study, and for countless hours of helpful scientific discussions, proofreading, editing, and career counseling. In particular, I wish to thank him for allowing me to explore the field of oceanography to the fullest by giving me the independence as well as the guidance I needed, and by encouraging my travels to many leading institutions.

I also wish to thank the members of my thesis committee, Paola Rizzoli, Heidi Nepf, John Trowbridge, Steve Lentz, and Karl Helfrich, for always making their time available when help was needed. Particular thanks go to John Trowbridge and Steve Lentz, whose scientific insight and ideas have greatly contributed to the content of this thesis. I am also grateful to Nelson Hogg and the chairman of the defense, Jim Price, for numerous helpful discussions and suggestions. Glen Gawarkiewicz and Kurt Polzin have not only been a source of valuable scientific advice, but have been readily volunteered their time to discuss life after graduate school on more than one occasion.

The US. GLOBEC Northwest Atlantic/Georges Bank 1995 Stratification Study was possible only through the commitment and diligence of many principal investigators and their co-workers. I especially wish to thank Sandy Williams and his team for collecting the BASS tripod data, and Steve Lentz, Bob Beardsley, Bob Weller, Jim Manning and their teams for obtaining the moored oceanographic and meteorological data. All participants made their data freely available and gave me the opportunity to direct my thesis into any area I wished to choose. Thanks are also due to Janet Fredericks, Jim Churchill and Tom Gross for their assistance with BASS data processing, Neil Oakey, Dave Hebert and their co-workers for collecting the microstructure data and making the data available, Nancy Brink and Nan Galbraith for processing the moored data, and Carol Alessi for her help with managing the data set. Chris Naimie gave valuable insight into the Dartmouth circulation model and readily made numerical results available for comparison to observation. Julio Candela provided the version of the M.G.G. Foreman tidal analysis code used in this study. The MY $2.51-\mathrm{D}$ numerical circulation model was made available by Stephen Monismith and is based on the Blumberg-Mellor 3-D hydrodynamic 
circulation model.

The support and friendship of many people in and outside the Joint Program have made my experience as a graduate student a pleasurable one. Thanks to Anne-Marie Michael for helping me with all kinds of editing problems, and for being an understanding housemate (and landlady) throughout the busy last year of my graduate student career. Many thanks also to Jubao Zhang, Jamie Pringle, Derek Fong, Melissa Bowen, Jay Austin, and Ken Koga for their valuable insight as well as for being good friends. The wonderful music lessons with Sara Hoff have made my life richer, and often helped me to see my scientific work from a new, fresh perspective. And finally, a special thank you to Mario Sengco for sharing hours and hours of good music, conversation, and food.

The research presented in this thesis was generously supported by the National Science Foundation under grants OCE 93-13671 and OCE 96-32357 as part of the U.S. GLOBEC/Georges Bank Program. 


\section{Contents}







2. Observations and Modeling of the Tidal Bottom Boundary Layer, Southern Flank of Georges Bank.

Part I: Nearly Homogeneous Conditions . . . . . . . . . . . . . . 14











2.3.1 ST1 Surface and Subsurface Mooring $\ldots \ldots \ldots \ldots \ldots \ldots$

2.3.2 ST1 Benthic Acoustic Stress Sensor (BASS) Tripod .........21





2.4.1 Investigation Period $\ldots \ldots \ldots \ldots \ldots \ldots \ldots \ldots \ldots \ldots \ldots \ldots \ldots \ldots \ldots \ldots \ldots \ldots \ldots \ldots$ 
2.4.2 Temperature, Salinity, and Density Fields ..............23

2.4.3 Richardson Number Variation $\ldots \ldots \ldots \ldots \ldots \ldots \ldots \ldots \ldots \ldots \ldots$



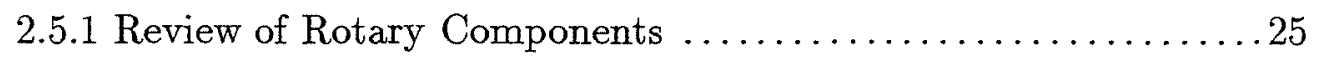



2.5.3 Boundary Layer Thickness $\ldots \ldots \ldots \ldots \ldots \ldots \ldots \ldots \ldots \ldots$





2.6.2 Logarithmic Fits . . . . . . . . . . . . . . . . . . 31





2.6.5 Bottom Stress Estimates from the $M_{2}$ Velocity Defect ......34



2.7.1 Performance of the 2 LK Model . ....................... 35

2.7.2 Performance of the MY2.5 Model .................... 36

2.7.3 Discussion of the MY2.5 Model .................... 38





Appendix B: The Two-Layer- $K(2 \mathrm{LK})$ Model $\ldots \ldots \ldots \ldots \ldots \ldots \ldots 44$

Appendix C: The Mellor-Yamada Level 2.5 (MY2.5) Model ..........46








Figures

3. Observations and Modeling of the Tidal Bottom Boundary Layer, Southern Flank of Georges Bank.

Part II: Strongly Stratified Conditions $\ldots \ldots \ldots \ldots \ldots \ldots \ldots \ldots \ldots \ldots$











3.4 Shelf-Slope Front (SSF) Intrusion (May 6-23) $\ldots \ldots \ldots \ldots \ldots \ldots \ldots$

3.4.1 Bottom-Trapped Phase (May 6-14) .................. 84

3.4.2 Surface-Intensified Phase (May 14-22) ................ 88

3.4.3 The $M_{2}$ Current Ellipse .......................... 89

3.4.4 Numerical Model ............................... 91

3.4.5 Model-Data Comparison $\ldots \ldots \ldots \ldots \ldots \ldots \ldots \ldots \ldots \ldots . \ldots . \ldots . \ldots 2$





3.5.2 Model-Data Comparison $\ldots \ldots \ldots \ldots \ldots \ldots \ldots \ldots \ldots \ldots \ldots \ldots$

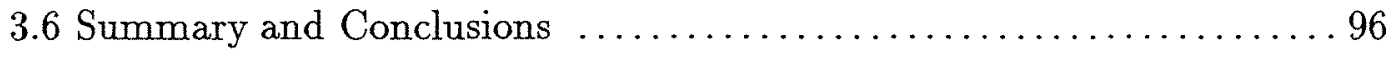



Acknowledgments ........................................ 101






Figures

4. The Surface Boundary Layer on the Southern Flank of Georges Bank ....................................... 136





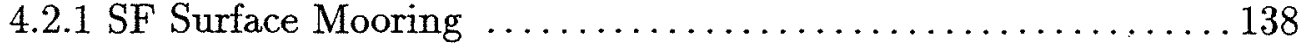

4.2.2 Subtidal Momentum Equations . .................... 138

4.2.3 Estimation of Subtidal Momentum Terms ............... 141

4.2.4 Subtidal Momentum Balance ................... 142

4.2.5 Comparison to Numerical Circulation Models ............. 145

4.2.6 Comparison of Wind and Bottom Stress ............. 147

4.2.7 Surface-Mixed Layer Depth $\ldots \ldots \ldots \ldots \ldots \ldots \ldots \ldots \ldots \ldots$

4.3 The Wind-Driven Circulation $\ldots \ldots \ldots \ldots \ldots \ldots \ldots \ldots \ldots \ldots \ldots \ldots \ldots \ldots \ldots \ldots \ldots \ldots$

4.3.1 Estimation of the Wind-Induced Currents .............. 151















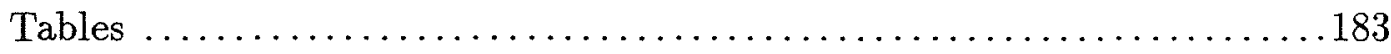



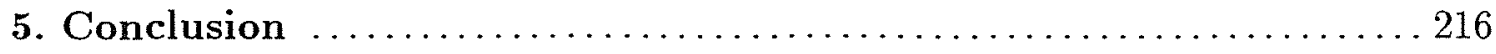




\section{Chapter 1}

\section{Introduction}

The research presented in this thesis is part of the US GLOBEC (GLObal ocean ECosystem dynamics) Northwest Atlantic/Georges Bank program, an interdisciplinary project whose main objective is to investigate and understand the key physical and biological processes which influence the distribution and abundance of four target species (larval cod and haddock, and their zooplankton prey the copepods Calanus finmarchicus and Pseudocalanus) on Georges Bank and in the Gulf of Maine. Georges Bank was chosen as the first GLOBEC study site, since it represents a region of high biological productivity and is thought of as a semi-enclosed ecosystem.

Georges Bank is a shallow submarine bank located between the deeper Gulf of Maine and the continental slope (Fig.2.1). The water depth varies from an average of $50 \mathrm{~m}$ on the bank plateau to $200 \mathrm{~m}$ in the Gulf of Maine, and $50-100 \mathrm{~m}$ on the southern flank of the bank bordering the continental slope. Two factors are largely responsible for the high biological productivity in this region: first, strong semidiurnal tidal flow creates intense turbulent mixing, which is caused by the interaction of the tidal currents with the rough bottom topography. As a result, the crest of the bank is well mixed at all times (Hopkins and Garfield, 1981). Tidally-induced turbulent mixing accounts for rapid availability of regenerated nutrients within the photic zone, resulting in large primary production rates in the Bank's central area (O'Reilly and Busch, 1984). In spring and summer, a tidal mixing front develops that separates the homogeneous water on the bank plateau from the stratified regions over the outer bank where turbulent mixing is limited by buoyancy forcing. Recently, patches of enhanced "new" primary production (i.e., primary production based on inorganic nitrogen) have been identified at the tidal mixing front (e.g., 
Horne, et al., 1996), indicating cross-frontal mixing may play a fundamental role in maintaining the Bank's biological productivity. In addition to causing large primary production rates which in turn induce productivity on higher trophic levels, tidal mixing processes also affect the abundance of ground fish in a direct manner: during their early life stages, stocks of cod and haddock larvae are confined to the southern flank of the bank, where they are advected southwestward by the mean circulation (e.g., Werner et al., 1996). The survival rate of the larval fish is a function of prey encounter rate and is strongly dependent on turbulent mixing.

The second factor which greatly affects productivity in the Georges Bank region involves the clockwise around-bank circulation that is superimposed on the tidal flow (e.g., Limeburner and Beardsley, 1996). Of particular interest to the fisheries is the flow field on the southern flank of the bank, where cod and haddock larvae spend their early life stages and are strongly impacted by advective processes. In addition, the around-bank circulation has a retaining effect on the water mass constituting the crest of the bank, including its inherent chemical and biological properties. However, it is important to realize that the Bank is far from representing a closed system: cross-bank exchange can occur through various mechanisms, e.g., tidal dispersion, nonlinear internal wave features, Gulf Stream rings, and wind-induced currents. Especially wind-forcing may have pronounced effects on the Georges Bank physics and ecology, e.g., by causing current transports that reduce the biomass in the bank area, possibly advecting fish larvae from the southern flank into the open ocean. Previous results from a coupled physical-biological model have demonstrated wind forcing can strongly impact the Bank's zooplankton dynamics, indicating interannual variability in surface wind stress may explain variations in observed zooplankton abundance for different years (Lewis et al., 1994). Despite initial modeling efforts, the exact implications of wind forcing on the Bank's biology are only poorly understood, largely because the flow field's response to surface winds demands further investigation.

It follows from the description of the bio-physical interactions given above that a detailed examination of the friction-induced turbulent mixing processes as well as the around-bank circulation is necessary in order to understand the ocean physics and ecosystem dynamics in the Georges Bank region. In this thesis, emphasis is placed on the investigation of the tidally driven bottom boundary layer and the wind-induced circulation on the southern flank of Georges Bank. The thesis makes extensive use of moored array data taken from February-August 1995 at study sites 
located on the 69- and 76-m isobaths between the bank plateau and the continental slope. The central mooring (76-m water depth) was equipped with a meteorological station, current meters, and temperature as well as conductivity sensors distributed throughout the water column. Two sets of Benthic Acoustic Stress Sensor (BASS) tripod measurements were taken from February-April and July-August and provided additional information about the velocity and temperature distribution near the bottom.

The data set used in this study presents a unique opportunity to investigate several of the important aspects of the Georges Bank circulation. The length of the deployment period allows for the examination of seasonal variations in the flow and density fields, and observations can be brought into context with the present understanding of the physics on the southern flank of Georges Bank. Due to the high vertical resolution of the measurements, particularly near the bottom and surface, a detailed analysis of the tidal bottom boundary layer and wind-driven surface layer could be performed. BASS measurements were especially useful to infer bottom friction parameters such as bottom stress, and to relate these parameters to the observed bottom boundary layer dynamics. For the first time, the vertical distribution of the tidal currents and observational estimates of bottom stress could be compared in detail to results from numerical models. Through this comparison, valuable insight was gained into the performance of commonly used turbulence closure schemes during stratified and unstratified conditions. Clearly, the results of the bottom boundary layer analysis are beneficial to the better understanding of tidal mixing processes not only on Georges Bank, but in continental shelf and estuarine environments in general. In addition, the possibility to infer lateral density gradients from the moored array measurements together with the velocity data taken at the central mooring site allowed for the separation of the wind-induced current distribution from the background flow field in a manner that has previously been reserved to open ocean studies.

Apart from the introduction, the thesis comprises four additional chapters covering the following topics: Chapter 2 investigates the vertical structure of the semidiurnal tidal bottom boundary layer during nearly homogeneous conditions, i.e., during conditions typical of winter. Chapter 3 extends the analysis from Chapter 2 to strongly stratified conditions representative of late spring and summer. Both Chapters 2 and 3 are based on results from observational as well as numerical anal- 
ysis. They appear in the thesis as partially revised versions of manuscripts that were submitted for publication by Werner and Beardsley ${ }^{1}$. The following chapter, Chapter 4, describes and discusses the observations of the wind-driven circulation on the southern flank of Georges Bank. Each of the Chapters 2-4 stands on its own, and contains an introduction and conclusion section specifically designed to match the individual chapter's contents. A final conclusion section summarizing the most important results is added in Chapter 5. Quantitative expressions are numbered from (1) upward in each chapter, since cross-referencing to equations listed in other chapters does not occur. References, appendices, figures, and tables are attached at the end of the chapters.

\section{References}

Hopkins, T. S., and N. Garfield III, Physical Origins of Georges Bank water. J. Marine Res., 37, 103-139, 1981.

Horne, E.P.W., J.W. Loder, C.E. Naimie, and N.S. Oakey, Turbulence dissipation rates and nitrate supply in the upper water column on Georges Bank. Deep Sea Res., Part II, 43, 1683-1712, 1996.

Lewis, C.V.W., C.S. Davis, and G. Gawarkiewicz, Wind forced biological-physical interactions on an isolated offshore bank. Deep Sea Res., Part II, 41, 1547$1574,1994$.

Limeburner, R., and R.C. Beardsley, Near-surface recirculation over Georges Bank, Deep Sea Res., Part II, 43, 1547-1574, 1996.

O'Reilly, J.E., and D. A. Busch, Phytoplankton primary production on the northwestern Atlantic shelf. Symposium on the biological productivity of north Atlantic shelf areas, Kiel, Germany, Mar.2-5, 1982, Rapp P-V Reun Cons Int Explor Mer., 183, 255-268, 1984.

Werner, F.E., R.I. Perry, R.G. Lough, and C.E. Naimie, Trophodynamics and advective influences on Georges Bank larval cod and haddock. Deep Sea Res., Part II, 43, 1793-1822, 1996.

\footnotetext{
${ }^{1}$ Robert C. Beardsley, Department of Physical Oceanography, Woods Hole Oceanographic Institution, Woods Hole, MA 02543, USA
} 


\section{Chapter 2}

\section{Observations and Modelling of the Tidal Bottom Boundary Layer, Southern Flank of Georges Bank. Part I: Nearly Homogeneous Conditions.}

\section{Preface}

Chapter 2 represents a partially revised version of a manuscript that was submitted for publication by Werner and Beardsley. A limited part of the research described in Chapter 2 was carried out in the context of Werner's (1996) master thesis. The master thesis covered the basic analysis of the bottom tripod data and results from the tidal decomposition, as well as a first comparison of the semidiurnal velocity profiles to numerical model results. In the framework of the $\mathrm{Ph}$. $\mathrm{D}$. thesis, a comprehensive literature search was carried out to identify previous related studies, general descriptions of the water structure and impacts of stratification on turbulent mixing were added, the scaling of the tidal boundary layer thickness was revised and extended, the bottom tripod data were reprocessed to eliminate an earlier error and the bottom friction parameters were rederived, the model-data comparison was extended to address the effects of wind forcing and weak stratification, and the results were summarized in a form suitable for publication. 


\begin{abstract}
We examine the vertical structure of the tidally-driven bottom boundary layer (TBL) during nearly homogeneous conditions representative of winter in a shallow coastal region dominated by semidiurnal tides, i.e., the southern flank of Georges Bank. From moored current meter and bottom-tripod mounted acoustic travel time measurements, we infer current profiles and bottom stress estimates at a 76-m deep study site. The observed velocity distribution is brought into context with analytical predictions and scaling arguments for tidal flow in the presence of bottom friction. Richardson number estimates indicate turbulence is sustained by tidal current shear in the TBL, while wind-driven currents play a role in and above the weak winter pycnocline.

Current measurements and bottom stress estimates are compared to numerical results from two one-dimensional models: a two-layer eddy viscosity model with linear eddy viscosity distribution in the lower and constant eddy viscosity in the upper layer, and a continuous eddy viscosity model with Mellor Yamada level 2.5 (MY2.5) closure. Both models compare favorably with the observations. Good agreement between the MY2.5 model results and data strongly depends on the specification of vertical stratification and wind forcing.
\end{abstract}

\title{
2.1 Introduction
}

Tidal currents represent an important component of the coastal circulation over wide areas of the continental shelf. In many coastal and estuarine regions, the interaction of tidal flow with rough bottom topography accounts for the major part of the turbulence production at the lower boundary. The result is the formation of a tidally-driven bottom boundary layer (TBL), i.e., a region of significant mixing where turbulence production is sustained by flow shear. Velocity shear is greatest near the bottom, and decreases upward as the top of the TBL is approached. The TBL thickness varies depending on current strength, bottom roughness, and vertical stratification.

Dimensional analysis shows the velocity distribution near a rough surface in the absence of stratification is logarithmic for steady non-rotational (Clauser,1956), steady planetary (Tennekes, 1973), and rectilinear oscillating flows (Grant and Mad- 
sen, 1986). The vertical extent to which the logarithmic approximation is valid depends on requirements about the goodness of the logarithmic fit, and is typically taken as $\frac{1}{10}$ of the total boundary layer-thickness in steady flows (Clauser, 1956; Grant and Madsen, 1986). In the case of a rectilinear tide, Soulsby and Dyer (1981) found the law of the wall needs to be modified to account for effects of tidal acceleration and deceleration. Gross and Nowell (1983) presented limited observational evidence supporting Soulsby and Dyer's (1981) results, but concluded uncertainties of turbulence measurements and logarithmic fits are too large to justify refinements in turbulent boundary layer theory.

In steady geophysical flows, bottom friction is balanced by the Earth's rotation, leading to cyclonic veering of the velocity vector toward the bottom (Ekman, 1905). Similar physics apply to oscillating flows in the absence of rotation, where the veering of the velocity vector is replaced by the phase lead of the near-bottom currents (Grant and Madsen, 1986). In the case of tidal currents, both the Earth's rotation and tidal acceleration combine to balance the bed shear stress. The results are phase advance of the near-bottom flow, and rotation of the tidal ellipse toward the bottom (Prandle, 1982a).

Observations of tidal current profiles frequently focus on estuarine environments and river plumes. In such regions, the tidal flow tends to be strongly polarized, with the major axis oriented in the cross-shelf direction at the river mouth (e.g., Beardsley et al., 1995), and along the coastline in the region of fresh water influence (ROFI) (e.g.,Visser et al., 1994). Previous investigators found semidiurnal tidal ellipses in the Rhine ROFI are almost degenerate near the surface during wellmixed conditions, and display weak anticlockwise circulation at greater depths as suggested by theory (Visser et al., 1994; Souza and Simpson, 1996). These results are consistent with observations of nearly rectilinear tidal flow in the central North Sea away from the direct influence of riverine freshwater input (Maas and van Haren, 1987). Observations of rotating semidiurnal tidal currents have been presented by Soulsby (1983) for the Celtic Sea, also displaying qualitative agreement with analytical predictions.

Analytical investigation of periodically oscillating flows requires the a priori specification of eddy viscosity profiles, such as vertically constant (Sverdrup, 1927; Prandle, 1982a,b), linear (Prandle, 1982a; Soulsby, 1983), and linear-constant profiles (Kagan, 1966; Trowbridge and Madsen, 1984). Trowbridge and Madsen's (1984) 
linear-constant eddy viscosity model compares well with laboratory results from Jonsson and Carlsen (1976) for homogeneous rectilinear flow in the absence of rotation. Soulsby's (1983) observations suggest the vertical extent of the tidal boundary layer lies between scale heights derived for constant and linear eddy viscosity distributions, if the water column is unstratified. Comparison of model results using time-dependent and steady eddy viscosity parameterizations indicates temporal variation of eddy viscosity has little effect on the first harmonic of the predicted flow (Trowbridge and Madsen, 1984; Davies, 1990). The implementation of a timevarying eddy viscosity in analytical and numerical models is of greater significance to the accurate prediction of bed shear stress and higher order harmonics of the flow field (Lavelle and Mofjeld, 1983; Trowbridge and Madsen, 1984; Davies, 1990).

Numerical models of tidal flow over rough bottom topography frequently involve advanced turbulence closure schemes, which are based on the turbulent kinetic energy equation and prognostic or diagnostic expressions for a turbulent mixing length (e.g., Davies and Jones, 1990; Chen, 1992; Simpson and Sharples, 1994; Naimie, 1995; Simpson et al., 1996). An alternative and computationally more efficient approach is the use of spectral models, which incorporate eddy viscosity parameterizations with specified vertical distribution and time-dependence that is related to the flow field. Davies and Xing (1995) found results from a spectral model with vertically constant eddy viscosity are close to those from turbulence energy models for homogeneous tidal flow. Similar conclusions were drawn earlier by Davies (1991), who showed deviations in current profiles predicted by models from either group are less than those due to different mixing length formulations in turbulence energy models. Comparison to limited observation suggests spectral and turbulence energy models reproduce the main features of the flow field reasonably well (e.g., Davies and Jones, 1990; Davies and Aldridge, 1993), although not much is known about their performance in the highly turbulent near-bed region of the TBL.

Here, we examine the vertical structure of the bottom boundary layer (BBL) during nearly homogeneous conditions in a shallow coastal region dominated by the semidiurnal tides, i.e., the southern flank of Georges Bank. The use of an extensive observational data set with velocity measurements in and above the bed shear layer distinguishes our study from previous work. Observations combine moored temperature, conductivity, and vector measuring current meter data at a 76-m deep study site with measurements from bottom tripod-mounted acoustic travel time current 
meters. From our data, we are able to obtain velocity profiles as well as estimates of bottom friction parameters and bed shear stress. We compare our observations to results from two one-dimensional numerical models: a linear-constant eddy viscosity model and a turbulence energy model with Mellor-Yamada level 2.5 closure. Emphasis is placed on the ability of the models to accurately predict the observed velocity profile and bed shear stress. The impact of strong stratification on TBL dynamics at the same location is discussed in a subsequent paper (part II).

\subsection{Physical Setting}

Georges Bank is a shallow submarine bank located between the deeper Gulf of Maine and the continental slope (Fig.2.1). The basin geometry of the Gulf of Maine and adjacent Bay of Fundy is near quarter-wave resonance with the oceanic semidiurnal tidal forcing (Garrett, 1972), causing large tidal transports across Georges Bank (Brown, 1984). Over the southern flank and most of the bank plateau, the $M_{2}$ currents carry more than $85 \%$ of the kinetic energy in the flow field (Table 2.1). At the 76-m deep study site on the southern flank (ST1, Fig.2.2), typical depthaveraged $M_{2}$ velocities are $41(26) \mathrm{cm} \mathrm{s}^{-1}$ along the major (minor) axis of the current ellipse. The ellipse orientation is along the $x$-axis and roughly perpendicular to the local isobaths (Fig.2.2), where $+x$ is aligned with $330^{\circ} \mathrm{T}$ (Brown, 1984). Inclination and eccentricity of the $M_{2}$ current ellipse are indicative of a Sverdrup plane wave that propagates on-bank from the open ocean. Amplitude modulations of the semidiurnal currents due to spring-neap variability are about $\pm 12 \mathrm{~cm} \mathrm{~s}^{-1}$ and $\pm 5 \mathrm{~cm} \mathrm{~s}^{-1}$ for the large and small spring-neap cycles, respectively.

The density distribution on Georges Bank is determined by meteorological forcing, bottom friction, and horizontal gradients due to adjoining water masses. In summer, the competing effects of bottom friction-induced turbulent mixing and surface heating manifest as a tidal mixing front (TMF) surrounding the bank near the 60-m isobath (Fig.2.2 and 3). Inside the TMF lies a water mass termed Georges Bank Water (GBW) (Hopkins and Garfield, 1981), which is distinguished in all seasons by its vertical and horizontal homogeneity (Fig.2.3). Characteristic temperatures and salinities of the GBW change from 3-16 $\mathrm{C}$ and 33.0-32.2 from winter through summer (Flagg, 1987). Approximately $50 \mathrm{~km}$ to the south of the TMF, the 
Shelf-Slope front (SSF) marks the boundary from fresh Shelf Water to saline Upper Slope Water (USW) with salinities 35-36. The base of the SSF is located near the 100-m isobath. Density gradients across the SSF are weak in winter, when temperature and salinity tend to compensate for one another. In summer on the shelf-side of the front, the offsetting effect of temperature are greatly reduced below the seasonal thermocline. As a result, the off-bank density gradient intensifies underneath the density interface, so that the salinity front coincides with a density front. Above the thermocline, colder temperatures on the crest of the bank outweigh the effects of salinity, resulting in increasing densities on-bank.

The vertical and horizontal density distribution between the TMF and SSF is subject to large seasonal variations (Flagg, 1987). In winter, convective overturning and mixing by tides and winter storms result in nearly vertical isopycnals. Over wide parts of the southern flank, weak vertical stratification is maintained in the upper water column by buoyant water from the Scotian Shelf, which originates in the Labrador Current and the Gulf of St. Lawrence (Chapman and Beardsley, 1989). Cross-bank gradients on the southern flank are weak in winter, and may be on- or off-bank depending on location. In summer, a seasonal thermocline develops, and the transition region between the TMF and SSF becomes vertically stratified.

Throughout the year, a clockwise mean circulation is present, driven by tidal rectification over the sloping bottom (Loder, 1980; Loder and Wright, 1985; Tee, 1985; Chen, 1992; Butman et al., 1983), upstream buoyancy sources such as the Labrador Current and the St. Lawrence River (Chapman and Beardsley, 1989), and, especially in winter, wind forcing. Previous investigators have shown the seasonal intensification of the SSF is in geostrophic balance with enhanced vertical along-bank shear (Butman et al., 1987). CTD cross-bank sections reveal depthaveraged thermal wind currents over the southern flank are about $2 \mathrm{~cm} \mathrm{~s}^{-1}$ at the end of winter and $10 \mathrm{~cm} \mathrm{~s}^{-1}$ in late summer (Butman et al., 1987).

\subsection{Moored Array}

As part of the U.S. GLOBEC Northwest Atlantic/Georges Bank 1995 Stratification Study, moored current, temperature, and conductivity data were taken on the southern flank of Georges Bank (Fig.2.4). Measurements were made Febru- 
ary 3-August 23 on the 76-m isobath at Stratification Site 1 (ST1, 40 $51.8^{\prime} \mathrm{N}$, $67^{\circ} 33.5^{\prime} \mathrm{W}$ ), located about $20-30 \mathrm{~km}$ on-bank of the SSF and $20 \mathrm{~km}$ off-bank of the TMF, and February 3-August 4 on the $69-\mathrm{m}$ isobath at Stratification Site 2 (ST2, 40 $57.4^{\prime} \mathrm{N}, 67^{\circ} 37.6^{\prime} \mathrm{W}$ ), located about $12 \mathrm{~km}$ on-bank of ST1 (Fig.2.2). The bottom slope at both locations was $\approx 8 \cdot 10^{-4}$. Bottom sediments at both sites were medium-to-coarse sand, with median grain size $0.25-1 \mathrm{~mm}$.

The moored array at $\mathrm{ST} 1$ consisted of a surface and a subsurface mooring separated by $\approx 260 \mathrm{~m}$ along-bank distance, with the surface mooring in the northernmost position. During February 3-April 4, a Benthic Acoustic Stress Sensor (BASS) tripod was deployed on the seafloor $\approx 100 \mathrm{~m}$ to the southwest of the subsurface mooring, providing measurements of near-bottom velocities and temperatures. A second set of BASS measurements was taken July 11-August 23 and is discussed in Part II. Measurements at ST2 focused on the upper water column (Fig.2.4) and are used here to determine the direction of cross-bank salinity, temperature and density gradients.

A brief summary of the moored instrumentation at ST1, the BASS tripodinstrumentation at ST1, and the moored instrumentation at ST2 are given next.

\subsubsection{ST1 Surface and Subsurface Mooring}

The surface mooring was equipped with eight Vector Measuring Current Meters (VMCMs, sample rate $7.5 \mathrm{~min}$ ) which measured horizontal currents and temperature at nominal heights of $71,68.5,66,62,57,51,44.5$, and $39 \mathrm{~m}$ above bottom (mab), four internally recording conductivity/temperature instruments (SeaCATs, sample rate $1.5 \mathrm{~min}$ ) at nominal heights $74.5,70,65$ and $50 \mathrm{mab}$, six temperature loggers (TPODs, sample rate $30 \mathrm{~min}$ ) at nominal heights $63,59,53.5,47,41.5$, and $35 \mathrm{mab}$, and a Miniature Temperature Recorder (MTR, sample rate $30 \mathrm{~min}$ ) at nominal height 72.5 mab (Fig.2.4). Meteorological data were taken by a Vector Averaging Wind Recorder (VAWR, sample rate $15 \mathrm{~min}$ ) and an Improved Meteorological Recorder (IMET, sample rate $1.0 \mathrm{~min}$ ), both mounted on the $3-\mathrm{m}$ discus buoy supporting the subsurface instruments. The instrumentation of the subsurface mooring consisted of five VMCMs at $30.5,24,18,12$, and 6 mab, two SeaCATs at 29.5 and $11 \mathrm{mab}$, and two TPODs at 14.5 and $8.5 \mathrm{mab}$.

No or bad data were recovered from the VMCM current and temperature units at $24 \mathrm{mab}$, the VMCM current unit at $51 \mathrm{mab}$, the VMCM temperature units at 6 
and $18 \mathrm{mab}$, and the TPODs at 59 and $41.5 \mathrm{mab}$. The VMCM velocity record at 18 mab ended on May 21 and contained spikes, which were removed for the purpose of data analysis.

VMCM measurement errors may be as large as $\pm 2 \mathrm{~cm} \mathrm{~s}^{-1}$, with compass uncertainties $\pm 5^{\circ}$ (Lentz, personal communication). Accuracies of temperature and conductivity measurements are $\pm 0.005^{\circ} \mathrm{C}$ and $\pm 5 \cdot 10^{-3} \mathrm{~S} \mathrm{~m}^{-1}$, respectively (Lentz, Limeburner, personal communication), where $0.1 \mathrm{~S} \mathrm{~m}^{-1}$ translates roughly into 1 PSU. Based on temperature-salinity diagrams representative of winter, these values yield uncertainties of $\pm 0.02 \sigma_{\theta}$ for potential density. Consistent offsets due to calibration error were found in some of the temperature and conductivity records. Temperature biases $<0.015^{\circ} \mathrm{C}$ were removed from VMCM and TPOD temperature data to obtain a smooth temperature profile for nearly homogeneous conditions (Lentz, personal communication). Conductivity data at 70 mab were corrected for measurement bias $10^{-3} \mathrm{~S} \mathrm{~m}^{-1}(\approx 0.01 \mathrm{PSU})$.

\subsubsection{ST1 Benthic Acoustic Stress Sensor (BASS) Tripod}

The BASS tripod was equipped with five acoustic travel time current meters (Williams et al., 1987) at $0.24,0.60,1.20,2.55$, and $4.45 \mathrm{~m}$ above deck (pods 1-5), eight thermistors at $0.24,0.62,1.22,1.90,2.53,3.24,4.43$, and $5.72 \mathrm{~m}$ above deck (thermistors 1-8), and five Optical Backscatter Sensors (OBS) at 0.11, 0.67, 1.27, 2.45 , and $4.45 \mathrm{~m}$ above deck (Fig.2.5). Sensor heights above the bottom are $2 \mathrm{~cm}$ below the above-deck values to account for sinking of the tripod legs. A camera was mounted to one of the side strobes designed to take bottom photographs at eight-hour intervals.

Temperature, optical backscatter, and acoustic travel time were recorded at $2 \mathrm{~Hz}$ during bursts of 7.5-min length. One burst occurred every half hour, with centerpoints $3.25 \mathrm{~min}$ after the half and the full hour. In this study, only burst-averaged data are used.

Even with the best pre-deployment dock calibration to determine sensor velocity zeros, current profiles measured by BASS show consistent offsets relative to a bestfit logarithmic profile (Gross, personal communication). To correct for the offset residuals, Gross developed a procedure in which the logarithmic fit is found as a function of flow direction for all acoustic current meters. A sine wave is fitted to the average residuals and subtracted from the original data. This procedure was 
applied to the BASS data presented here.

Data were recovered from all tripod instruments, with the exception of thermistor 1. BASS thermistors were accurate to $\pm 0.001^{\circ} \mathrm{C}$. The precise measurement error of the acoustic current sensors is not known, but is expected to be significantly smaller than that of the VMCMs in the absence of flow distortion (Lentz and Butman, 1995). As a conservative estimate, we took the standard deviation within each burst as a representative uncertainty. The compass uncertainty of BASS is $\pm 2^{\circ}$ (Williams, personal communication). Inaccuracies in individual current sensor alignment up to about $5^{\circ}$ are possible.

\subsubsection{ST2 Surface Mooring}

The ST2 surface mooring was equipped with three SeaCATS (sample rate $7.5 \mathrm{~min}$ ) at 39,59 , and $68 \mathrm{mab}$, one Minilog temperature recorder (sample rate $60 \mathrm{~min}$ ) at $64 \mathrm{mab}$, three TPODs (sample rate $30 \mathrm{~min}$ ) at 31,44 , and $64 \mathrm{mab}$, two VMCMs (sample rate $7.5 \mathrm{~min}$ ) at 34 and $54 \mathrm{mab}$, and one Vector Averaging Current Meter (VACM, sample rate $7.5 \mathrm{~min}$ ) at $7 \mathrm{mab}$ (Fig.2.4). The VMCMs and VACM measured horizontal currents and temperature; an additional conductivity unit was attached to the VACM. Conductivity measurements at 39 and 59 mab were corrected to remove measurement bias $\leq 2 \cdot 10^{-2} \mathrm{~S} \mathrm{~m}^{-1}(\approx 0.2 \mathrm{PSU})$ (Lentz, personal communication).

\subsection{Water Structure}

\subsubsection{Investigation Period}

The period 1000 UT February 11 to 0000 UT March 11 was chosen for detailed analysis based on the following reasons:

a) Temperature was vertically homogeneous between 0.6-5.7 mab to less than $\pm 0.01^{\circ} \mathrm{C}$ (Fig.2.6), with thermal stratification that was $<0.002 \pm 0.001^{\circ} \mathrm{C}$ more than $97 \%$ of the time. These observations indicate the bed-shear layer was largely unaffected by vertical stratification.

b) Density was nearly homogeneous in the lowest $30 \mathrm{~m}$ of the water column, and only weakly stratified $\left(N^{2} \approx 10^{-5} \mathrm{~s}^{-2}\right)$ at greater heights (Fig.2.7). 
c) The length of the time series (27.6 days) allows for the full resolution of all semidiurnal tidal constituents, i.e., the $S_{2}\left(T_{S_{2}}=12.00 \mathrm{hrs}\right), M_{2}\left(T_{M_{2}}=12.42\right.$ $\mathrm{hrs})$, and $N_{2}\left(T_{N_{2}}=12.66 \mathrm{hrs}\right)$.

d) Amplitude modulation of the $M_{2}$ currents by the $N_{2}\left(S_{2}\right)$ astronomical forcing occurs over 14.8 (27.6) days, and completes approximately two cycles (one cycle) during the investigation period. Thus, effects of amplitude modulation on bottom stress and boundary layer thickness average out in the time mean.

\subsubsection{Temperature, Salinity, and Density Fields}

Time-mean temperature profiles indicate mean temperature differences between surface and bottom were $<0.1^{\circ} \mathrm{C}$ (Fig.2.7a). The density distribution is determined by the vertical structure of salinity (Fig.2.7b,c). Intermittent intrusions of low salinity water covered about $11 \%$ of the investigation period and caused vertical stratification $N^{2} \approx O\left(10^{-4}\right) \mathrm{s}^{-2}$ near the surface. A characteristic surface mixed layer depth for the remaining $89 \%$ of the investigation period is $6 \mathrm{~m}$ (Fig.2.7d).

Fig.2.8 shows SeaCAT measurements at 11-70 mab versus tidal flow direction $\alpha$. The tidal ellipse was divided into bins of $30^{\circ}$ width, and data within each bin were averaged to obtain one representative value. Fig. $2.8 \mathrm{~b}$ indicates temperature is lowest at the end of ebb when the tidal flow is along-bank $\left(\alpha=270^{\circ}\right)$, coinciding with largest salinity and potential density measurements (Fig.2.8c,d). Thus, cooler, denser, and saltier water is advected off-bank during ebb, while warmer, lighter, and fresher water is advected on-bank during flood. The observed temperature field is indicative of winter cooling causing lower temperatures on the bank plateau, while salinities reveal the influence of fresh Scotian Shelf water to the south of ST1. Characteristic variations of potential density between ebb and flood are $\Delta \sigma_{\theta}^{M_{2}}=$ $0.01-0.03$ (Fig.2.8d). Divided by the tidal excursion $\ell_{M_{2}}=\frac{2 U}{\omega_{M_{2}}}=6 \mathrm{~km}$, where $U=0.4 \mathrm{~m} \mathrm{~s}^{-1}$ is the cross-bank amplitude of the $M_{2}$ currents and $\omega_{M_{2}}=1.41 \cdot 10^{-4} \mathrm{~s}^{-1}$ the $M_{2}$ frequency, the on-bank density gradient at ST1 is $0.002-0.005 \sigma_{\theta} \mathrm{km}^{-1}$. These estimates are consistent with density differences inferred from measurements at ST1 and ST2. 


\subsubsection{Richardson Number Variation}

The Richardson number $R i=\frac{N^{2}}{U_{z}^{2}}$ was computed using stratification estimates from the SeaCAT data at 11, 29.5, 50,65, and $70 \mathrm{mab}$, and shear estimates $U_{z}$ from VMCM measurements at $12,18,30.5,39,45,57,62,66$, and 71 mab. Velocity shear was interpolated linearly with depth to obtain estimates at midpoints between adjacent SeaCATs.

Laboratory and geophysical observations show $R i<0.25$ is a useful criterion for the prediction of growing instabilities in stratified shear layers, in agreement with linear instability theory (Kundu, 1990). Using the critical value $R i_{c}=0.25$ to describe the transition from active to decaying turbulence requires the resolution of vertical scales approximately equal to the largest density overturns

$$
L_{B}=2 \pi\left(\frac{\epsilon}{N^{3}}\right)^{1 / 2}
$$

where $\epsilon$ is the rate of turbulent dissipation, and $\left(\epsilon / N^{3}\right)^{1 / 2}$ is the Ozmidov scale at which inertial and buoyancy forces balance (Gregg, 1993; Ozmidov, 1965). Rough estimates of $L_{B}$ can be obtained using dissipation rates from microstructure measurements taken in spring 1995 at ST1. On April 29, Oakey and Hebert measured $\epsilon \approx 10^{-7} \mathrm{~m}^{2} \mathrm{~s}^{-3}$ and $N^{2} \approx 10^{-5} \mathrm{~s}^{-2}$ at mid-depth immediately below the main pycnocline (see also GSO Rhode Island Tech. Report 96-6). Using (1), these estimates give $L_{B} \approx 11 \mathrm{~m}$, a value $4 \mathrm{~m}$ short of the distance between adjacent SeaCATs in the pycnocline where $N^{2} \approx 10^{-5} \mathrm{~s}^{-2}$ during our investigation period (Fig.2.7d). We therefore conclude overturning scales may be insufficiently resolved in the winter pycnocline, so that $R i_{c}=0.25$ has only limited meaning, and a larger critical value needs to be used. In the following we discuss results for both $R i_{c}=0.25$ and $R i_{\mathrm{c}}=0.5$.

Fig.2.9 shows vertical stratification and percentage of $R i<R i_{c}$ (circles) as a function of tidal flow direction defined in Fig.2.8a. Variation of $N^{2}$ is related to on-bank advection of more stratified water during flood, and off-bank advection of less stratified water during ebb. The result is larger $N^{2}$ for $\alpha=0-180^{\circ}$ than for $\alpha=180-360^{\circ}$ above the nearly homogeneous bottom mixed layer (Fig.2.9a). Intermittent intrusions of low salinity water distort the signal near the surface (last panel) . 
Uncertainties of $R i<R i_{c}$ were obtained by performing a Monte Carlo simulation. First, we computed representative uncertainties $\sigma_{N^{2}}$ and $\sigma_{U_{z}}$ assuming linear error propagation of random instrument noise. The uncertainty calculations were based on the previously listed measurements error of the unaveraged density and velocity data $\sigma_{\sigma_{\theta}}=0.02$ and $\sigma_{U}=2 \mathrm{~cm} \mathrm{~s}^{-1}$, respectively. Second, we generated time-series of normally distributed random noise with variance $\sigma_{N^{2}}^{2}$ and $\sigma_{U_{z}}^{2}$. Third, we added the generated noise to our estimates of $N^{2}$ and $U_{z}$, and determined the percentage $R i<R i_{c}$ for a given height and flow direction. Steps two and three were repeated 100 times. The mean of all 100 trials for $R i<R i_{c}$ is marked by circles, and the error bars correspond to the $95 \%$ confidence limit (Fig.2.9b,c).

Events $R i<R i_{c}$ are about $10 \%$ more frequent for $R i_{c}=0.5$ than for $R i_{c}=0.25$ everywhere but in the winter pycnocline, where results differ by as much as $25 \%$ (Fig.2.9b,c). Between 10-30 mab, $R i<R i_{c}$ more than $80 \%$ of the investigation period, indicating turbulence production is large in the BBL. Shear instability is also evident between $30-50 \mathrm{mab}$, where $R i<R i_{c} \quad 60-90 \%$ of the time. Between 50-65 mab where stratification is largest, vertical mixing is reduced, and $R i<R i_{c} \quad 15-55 \%$ of the investigation period depending on the choice of $R i_{c}$. At heights $>65 \mathrm{mab}, R i<R i_{c} \quad 70-100 \%$ of the time, indicative of a turbulent surface layer due to wind mixing.

Richardson numbers were also computed using shear estimates from high-passed currents with temporal variation $<33 \mathrm{hrs}$ (Fig.2.9b,c, diamonds). Results show $R i<R i_{c}$ events are reduced by about $10-20 \%$ in the pycnocline (50-65 mab) compared to computations based on total current shear. This behavior suggests subtidal currents with time scales greater than diurnal may contribute significantly to turbulence production at the density interface.

\subsection{Tidal Current Analysis}

\subsubsection{Review of Rotary Components}

The tidal decomposition was carried out on hourly-averaged VMCM and BASS velocity data using Godin's harmonic method (Foreman, 1978). Results are presented in counterclockwise $(+)$ and clockwise $(-)$ rotary components 


$$
\mathbf{R}^{ \pm}=R^{ \pm} e^{i \phi^{ \pm}}
$$

as defined by Soulsby (1983). The rotary components combine to describe the current vector according to

$$
u+i \cdot v=\sum_{j=1}^{M}\left(\mathbf{R}_{j}^{+} e^{i \omega_{j} t}+\mathbf{R}_{j}^{-} e^{-i \omega_{j} t}\right)
$$

where $u$ and $v$ are the velocity components in $x$-and $y$-direction, respectively, and $\omega_{j}$ are the radian frequencies of the resolvable tidal constituents. Current speeds along the major $(\mathrm{Maj})$ and minor $(\mathrm{Min})$ axes of the tidal ellipse can be computed from

$$
\begin{aligned}
& U_{M a j}=R^{+}+R^{-}, \\
& U_{M i n}=R^{+}-R^{-}
\end{aligned}
$$

where $U_{\min }>0$ denotes counterclockwise rotation of the velocity vector. Inclination and phase of the major axis with respect to the on-bank $(+x)$ reference axis are given by

$$
\theta=0.5 \cdot\left(\phi^{+}+\phi^{-}\right)
$$

and

$$
\phi=0.5 \cdot\left(\phi^{-}-\phi^{+}\right)
$$

respectively. Positive inclination denotes counterclockwise orientation from $+x$. The phase is evaluated at the center of the time series, here 0600 UT February 25, and gives the time $t=\frac{\phi}{\omega}$ to elapse before the velocity vector next passes the on-bank $(+x)$ reference axis.

Error limits are derived from the residual spectrum, i.e., the spectrum of the measured minus predicted currents. For the $M_{2}$ tide, the residual variance was summed over a frequency band centered at the $M_{2}$ frequency, divided by the band width $\pm 0.21 \cdot 10^{-4} \mathrm{~s}^{-1}$, and multiplied by 2 to give the standard error at 
the $95 \%$ confidence level. Error bars for $\phi^{+}, \phi^{-}$and $\theta$ are based on compass uncertainties and inaccuracies of BASS sensors alignment, and are $5^{\circ}$ and $7^{\circ}$ for the VMCM and BASS measurements, respectively. Instrument orientation has no impact on phase prediction, since both $\phi^{+}$and $\phi^{-}$are equally affected so that directional uncertainties cancel.

\subsection{2 $\mathrm{M}_{2}$ Currents}

$M_{2}$ current amplitudes show $R^{-}>R^{+}$at all heights, implying $U_{M i n}<0$ and clockwise rotation of the current ellipse (Fig.2.10a,d). For easier presentation, phases and inclination are depicted as differences with respect to the surface, with $\Delta \phi^{ \pm}$, $\Delta \phi$ and $\Delta \theta=0$ averaged over the topmost five VMCMs (Fig.2.10b,c,e,f). The inclination of the major axis near the surface is $2^{\circ} \pm 5^{\circ}$ counterclockwise from the on-bank $(+x)$ reference axis. Eccentricities $\epsilon=\frac{U_{M i n}}{U_{M a j}}$ range from $0.63-0.67$ between bottom and surface. With $\frac{f}{\omega_{M_{2}}}=0.67$, these results suggest $\epsilon \approx \frac{f}{\omega_{M_{2}}}$, indicative of a Sverdrup plane wave propagating across Georges Bank into the Gulf of Maine. This can be shown analytically from the linear momentum equations

$$
\begin{aligned}
& \frac{\partial u}{\partial t}-f v=-\frac{\partial p}{\partial x}+\frac{\partial \tau^{x}}{\partial z} \\
& \frac{\partial v}{\partial t}+f u=-\frac{\partial p}{\partial y}+\frac{\partial \tau^{y}}{\partial z}
\end{aligned}
$$

where $p$ and $\tau$ are pressure and stress divided by density, respectively. According to Brown (1984), (6) describe the flow field to lowest order. Using velocity data from ST1 and ST2, we estimate that the advective terms $u \frac{\partial u}{\partial x}$ and $u \frac{\partial v}{\partial x}$ are one order of magnitude smaller than the Coriolis and acceleration terms. This together with the assumption that along-bank variation of the flow field is negligible support Brown's (1984) conclusion that (6) represent a valid lowest order approximation. Writing (6) in rotary components gives for the $M_{2}$ constituent

$$
i\left(f \pm \omega_{M_{2}}\right) \mathbf{R}^{ \pm}=-\mathbf{P}^{ \pm}+\frac{\partial \tau^{ \pm}}{\partial z},
$$

where $\mathbf{P}^{ \pm}$and $\boldsymbol{\tau}^{ \pm}$are the rotary components of the pressure gradient and stress vector divided by density, respectively. The semidiurnal pressure gradient is barotropic 
to lowest order, since internal wave activity was not observed during the nearly homogeneous conditions presented here. Wind measurements show negligible variation in the semidiurnal frequency band (Fig.2.11), so that stress divergence is small above the BBL. As a result, $M_{2}$ current amplitudes are nearly constant in the upper water column (Fig.2.10a). Taking $\frac{\partial \boldsymbol{\tau}^{ \pm}}{\partial z}=0$ near the surface yields

$$
\mathbf{P}^{ \pm}=-i\left(f \pm \omega_{M_{2}}\right) \mathbf{R}_{\infty}^{ \pm}
$$

where the subscript $\infty$ denotes the free-stream above the BBL defined as the averaged currents measured by the topmost five VMCMs. Using (8), we compute $P_{M a j}=3.36 \pm 0.05 \cdot 10^{-5} \mathrm{~m} \mathrm{~s}^{-2}$ and $P_{M i n}=0.04 \pm 0.05 \cdot 10^{-5} \mathrm{~m} \mathrm{~s}^{-2}$ (Fig.2.12,dashed), with uncertainties resulting from the standard error of the tidal fit at the $95 \%$ confidence level. The orientation of $P_{M a j}$ is defined by the inclination of the current ellipse, and is approximately $2^{\circ} \pm 5^{\circ}$ from $+x$. Thus, the pressure forcing is nearly rectilinear in the cross-bank direction in agreement with $\epsilon \approx \frac{f}{\omega_{M_{2}}}$.

In the lower water column, bottom friction is balanced by current shear and increasing phase-lead of the velocity vector toward the bottom (Fig.2.10). With $\mathbf{R}^{+}$rotating counterclockwise and $\mathbf{R}^{-}$clockwise, phase-lead is defined by $\Delta \phi^{+}>$ $0, \Delta \phi^{-}<0$, and $\Delta \phi<0$. Near-bottom velocities lead the surface currents by about $12^{\circ} \pm 5^{\circ}$, corresponding to a phase advance of $0.4 \pm 0.2$ hours (Fig.2.10f).

The region of large current shear extends further away from the bottom for $R^{-}$than for $R^{+}$, in agreement with earlier observations in shelf seas and analytical predictions (Prandle, 1982a; Soulsby, 1983; Maas and van Haren, 1987) (Fig.2.10a). In a physical context, the counterclockwise rotation of $\mathbf{R}^{+}$assists Coriolis in balancing bottom friction, while the clockwise rotation of $\mathbf{R}^{-}$causes the opposite effect. Amplitudes of both rotary components display a weak maximum at $30.5 \mathrm{mab}$, which we attribute to measurement bias rather than physical processes. Our conclusion is based on subsequent analysis of the subtidal flow (not presented here), which indicates a $1-2 \mathrm{~cm} \mathrm{~s}^{-1}$ offset at the same height. In the upper water column, $R^{-}$amplitudes display weak shear between 45-57 mab. Closer investigation of several short subperiods did not indicate that enhanced shear in this region is related to intermittent intensification of the winter pycnocline. Thus, measurement bias $<2 \mathrm{~cm} \mathrm{~s}^{-1}$ is a more likely explanation. 
Similar to current amplitudes, phase angles $\Delta \phi^{ \pm}$indicate different boundary layer thicknesses for the clockwise and anticlockwise component (Fig.2.10b,c). The phase-lead $\Delta \phi^{+}$is almost indistinguishable from zero within error limits, with the exception of BASS measurements at 0.2 and $4.4 \mathrm{mab}$. On the other hand, $\Delta \phi^{-}$is different from zero at $45 \mathrm{mab}$ and below, showing increased phase-lead toward the bottom as suggested by theory. The result is clockwise rotation of the major axis with increasing depth $\left(|\Delta \theta|<11^{\circ} \pm 5^{\circ}\right)$, followed by weak counterclockwise veering near the bottom (Fig.2.10e).

\subsubsection{Boundary Layer Thickness}

Scale expressions for the vertical extent of the $M_{2}$ counterclockwise and clockwise boundary layers can be derived from (7). Substituting $\boldsymbol{\tau}^{ \pm}=K \frac{\partial \mathbf{R}^{ \pm}}{\partial z}$, where $K$ is an eddy viscosity, yields

$$
i\left(f \pm \omega_{M_{2}}\right) \mathbf{R}^{ \pm}=-\mathbf{P}^{ \pm}+\frac{\partial}{\partial z}\left(K \frac{\partial \mathbf{R}^{ \pm}}{\partial z}\right)
$$

Assuming no slip at the seafloor, solutions to (9) depend on assumptions about the magnitude, vertical distribution and temporal variation of $K$. For the simplest case $K=$ constant, $\mathbf{R}^{ \pm}$are described by the Ekman solutions for a rotating tide (Mofjeld, 1980; Kundu et al., 1981; Soulsby, 1983). The corresponding boundary layer scale heights are

$$
\delta^{ \pm}=c \cdot\left|\frac{K}{\omega_{M_{2}} \pm f}\right|^{1 / 2}
$$

where $c$ is an empirical constant. A more realistic eddy viscosity parameterization that accounts for the restriction of turbulent motion by the seafloor assumes the linear distribution

$$
K=\kappa u_{*} z
$$

where

$$
u_{*}=\sqrt{\tau_{b}}
$$


is friction velocity, $\tau_{b}$ is kinematic bottom stress (dynamic stress divided by density) , $\kappa$ is von Karman's constant, and $z$ is height above bottom. Expression (11) is in agreement with the law of the wall, which assumes a logarithmic velocity distribution near the bottom (Tennekes, 1973; Grant and Madsen, 1986). With (11), solutions to (9) give the boundary layer scale heights (Prandle, 1982a; Soulsby, 1983)

$$
\delta^{ \pm}=c \cdot \frac{\kappa \bar{u}_{*}}{\left|\omega_{M_{2}} \pm f\right|}
$$

where again, the constant $c$ is empirical, and $\bar{u}_{*}$ is a characteristic friction velocity, here taken as the mean friction velocity during one tidal cycle. Expressions similar to (13) were derived by Grant and Madsen (1986) for steady planetary flow $\left(\delta \simeq \frac{\kappa u_{*}}{f}\right)$ and non-rotational oscillatory flow $\left(\delta \simeq \frac{\kappa \bar{u}_{*}}{\omega}\right)$ based on scaling of the logarithmic velocity function describing the overlap layer. The Grant and Madsen (1986) derivation does not require a priori assumptions about the vertical structure of $K$, but infers from the logarithmic velocity distribution that (11) must hold very near the bottom where $\tau \approx \tau_{b}$. For a rotating tide, expression (13) can be obtained from the depth-integrated momentum equations assuming quadratic bottom drag consistent with the logarithmic law of the wall (Appendix A).

Due to the small amplitude of the counterclockwise component on Georges Bank, it is difficult to determine the thickness of the counterclockwise boundary layer and infer a characteristic ratio $\frac{\delta^{-}}{\delta^{+}}$from Fig.2.10. Using linear interpolation to estimate velocity amplitudes between current meters suggests that within error limits, $R^{+}$and $R^{-}$reach $80 \%$ of their near-surface values (i.e., averaged amplitude at topmost five VMCMs) at 2.2-7.8 mab and 9.5-11.5 mab, respectively. These values yield $\frac{\delta^{-}}{\delta^{+}}=1.2-5.8$, supporting either $\frac{\delta^{-}}{\delta^{+}}=\left[\frac{\omega_{M_{2}}+f}{\omega_{M_{2}}-f}\right]^{1 / 2}=2.3$ according to (10), or $\frac{\delta^{-}}{\delta^{+}}=\left[\frac{\omega_{M_{2}}+f}{\omega_{M_{2}}-f}\right]=5.2$ according to (13). Additional information about boundary layer thicknesses may be inferred from the vertical distributions of $\Delta \phi^{ \pm}$. Phase veering $\Delta \phi^{+}$is distinguishable from zero at BASS pod 4 (2.5 mab), with $\Delta \phi^{+} \geq 5^{\circ}$ within the error limits. The clockwise component displays more pronounced phase-lead toward the bottom, suggesting $\Delta \phi^{-} \leq-5^{\circ}$ at $18 \mathrm{mab}$ and below. Although the phase uncertainties do not allow for accurate prediction of the overall boundary layer thickness, these results give a rough estimate $\frac{\delta^{-}}{\delta^{+}} \approx 7$, in closer agreement with (13) than (10). 


\subsection{Bottom Friction}

\subsubsection{Logarithmic Layer}

In the absence of stratification, the velocity distribution near a rough surface follows the logarithmic law of the wall

$$
U=\frac{u_{*}}{\kappa} \ln \left(\frac{z}{z_{0}}\right)
$$

where $u_{*}, \kappa$ and $z$ are the same as in (11), $U$ is current speed, and $z_{0}$ is bottom roughness. Profiles of BASS and VMCM velocity data for February 11-March 11 are shown on a semi-logarithmic scale in Fig.2.13. Circles correspond to the average magnitude of all events with directions of near-bottom flow less than $\pm 45^{\circ}$ from the cross- $(a, b)$ and along-bank $(c, d)$ axes. Results from best-fit logarithmic profiles to BASS data at 0.2-2.5 mab (pods 1-4) were extrapolated throughout the water column to obtain current speeds $U_{f i t}$ (dashed). Using $\frac{\left|U_{f i t}-U\right|}{U_{f i t}}<5 \%$ as an arbitrary criterion to define the $\log$-layer thickness yields $z_{\log }=2.5,18,4.4$, and $2.5 \mathrm{mab}$ for current distributions centered around the $+x,-x,+y$, and $-y$ axes, respectively. The exceptionally thick logarithmic layer during off-bank flow conditions may be related to large measured off-bank currents in the subtidal frequency band, causing increased turbulence production when tidal and subtidal velocities add. Deviations of measured to best-fit profiles are $<5 \%$ for more than $80 \%$ of the investigation period at $0.2-2.5$ mab (pods 1-4), but less than $50 \%$ of the investigation period at $4.4 \mathrm{mab}$ (pod 5) and greater heights. The rapid growth of misfits above BASS pod 4 suggests a representative log-layer thickness is between 2.5-4.4 mab (pods 4-5).

\subsubsection{Logarithmic Fits}

Friction velocity $u_{*}$ and bottom roughness $z_{0}$ were estimated by taking the instantaneous best-fit logarithmic profiles to burst-averaged speeds $U$ at pods 1-4 (0.2-2.5 mab). Logarithmic fits with standard errors $>2.0 \mathrm{~cm} \mathrm{~s}^{-1}$ or deviations of estimated to measured currents $>2.0 \mathrm{~cm} \mathrm{~s}^{-1}$ could be indicative of flow distortion by tripod instrumentation (e.g., the camera) and were excluded from the analysis. This limits the data set to $89 \%$ of its original length from February 11March 11, with time-gaps that are rarely larger than 0.5-1 hour. Linear regression 
coefficients of all retained burst-averaged velocities at pods 1-4 to the logarithm of height above bottom are $\geq 0.95(\geq 0.90)$ for $79 \%$ (97\%) of the investigation period.

\subsubsection{Friction Velocity}

Fig.2.14a shows friction velocity as a function of time with missing values filled in by linear interpolation. The record average is $\bar{u}_{*}=1.2 \pm 0.4 \mathrm{~cm} \mathrm{~s}^{-1}$, where the standard deviation $\pm 0.4^{\circ} \mathrm{cm} \mathrm{s}^{-1}$ mostly describes semidiurnal variations. The $95 \%$ confidence intervals $\Delta u_{*}^{95}$ are of order $u_{*}$ (Fig.2.14b).

Friction velocities from logarithmic fits indicate a linear increase with current speed (Fig.2.15), in agreement with the quadratic drag law

$$
\tau_{b}=c_{D} U^{2}
$$

where

$$
c_{D}=\left[\frac{\kappa}{\ln \left(\frac{z}{z_{0}}\right)}\right]^{2} .
$$

is the quadratic drag coefficient. Least-squares fits of $u_{*}^{2}$ versus $U^{2}$ at pods $1-4$ give $c_{D}^{0.22 m}=3.62 \pm 0.26 \cdot 10^{-3}, c_{D}^{0.58 m}=3.14 \pm 0.18 \cdot 10^{-3}, c_{D}^{1.18 m}=2.62 \pm 0.14 \cdot 10^{-3}$, and $c_{D}^{2.53 m}=2.21 \pm 0.10 \cdot 10^{-3}$, respectively, where values denoted by \pm give the $95 \%$ confidence limits of the fits.

\subsubsection{Bottom Roughness}

Based on experimental results, the physical bottom roughness over a hydrodynamically fully rough boundary is $z_{0}=k_{b} / 30$, where $k_{b}$ is the equivalent sand grain roughness defined by the geometry of the dominant bedforms (Schlichting, 1968). Seafloor photographs reveal the presence of northeastward aligned sand ripples with height $\eta=1-2 \mathrm{~cm}$ and horizontal spacing $\lambda=15-20 \mathrm{~cm}$ (not shown). Using $k_{b}=30 \eta \cdot \frac{\eta}{\lambda}$ according to Grant and Madsen (1982) gives the characteristic physical bottom roughness $z_{0}=0.5-3 \mathrm{~mm}$.

On the shallow continental shelf, fluid motion near the bottom can be due to both currents and surface waves. If the wave orbital velocities are of similar magnitude as the current speeds, a wave boundary layer with characteristic thickness $l_{w}=$ 
$\frac{\kappa u_{* c w}}{\omega_{w w}}$ develops, where $u_{* c w}$ is the friction velocity due to both waves and currents and $\omega_{w}=$ is wave frequency (Grant and Madsen, 1986). The wave boundary layer represents a region of wave-induced turbulence and increases the apparent bottom roughness experienced by the current above it (Grant and Madsen, 1979). Estimates of wave orbital velocities can be computed from hourly surface wave data taken by the NDBC environmental buoy 44011 on the $88-\mathrm{m}$ isobath $\left(41^{\circ} 6^{\prime} \mathrm{N}\right.$, $\left.66^{\circ} 36^{\prime} \mathrm{W}\right)$. With characteristic wave heights $0.7-5.4 \mathrm{~m}$ and frequencies $\omega_{w}=0.5-$ $1.7 \mathrm{~s}^{-1}$ for February 11-March 11, typical wave velocities near the bottom are 0$11 \mathrm{~cm} \mathrm{~s}^{-1}$ at ST1 assuming energy conservation with no dissipation. The ratio of wave orbital velocities to burst-averaged current measurements at the lowest BASS sensor $(0.22 \mathrm{mab})$ is 0.18 in the time-average, indicating wave-induced turbulence does not play a dominant role but may intermittently increase the apparent bottom roughness. Relative magnitudes of burst variances to burst-averaged velocities also represent a measure for wave activity near the bottom. With a background level of $\approx 0.21$ during calm conditions, representative ratios for February 11-March 11 are $0.24 \pm 0.10$. Typical time scales of events with large velocity variance are a few hours long, again suggesting wave-induced turbulence is not a permanent feature, but occurs intermittently over short periods.

It is beyond the scope of this paper to present a detailed discussion about the effects of bedforms and surface waves on bottom roughness. Such analysis has been carried out for other regions, e.g. the northern California continental shelf using a BASS tripod specifically designed to study wave-current interaction (Grant et al.,1984). For our purposes, it is sufficient to make an order of magnitude prediction of $z_{0}$ to verify whether estimates from logarithmic fits are reasonable. Based on the wave boundary layer scaling and physical bottom roughness derived above, a representative length scale for $z_{0}$ is of order millimeters.

Estimates of bottom roughness were obtained by extrapolating the fitted logarithmic profiles to the zero intercept on the $\log (z)$ axis. Using current measurements between 0.2-2.5 mab, the extrapolation spans more than one decade, so that large scatter and great uncertainties are expected. Results for individual profiles range from $10^{-8} \mathrm{~mm}$ to $30 \mathrm{~mm}$, with $95 \%$ confidence limits of order $z_{0}$ (Fig.2.16). Histograms of $z_{0}$ display a wide peak centered around $1 \mathrm{~mm}$, in reasonable agreement with the order of magnitude estimate from bottom morphology.

Due to the wide scatter of $z_{0}$ values, it is difficult to infer an accurate estimate 
of bottom roughness based on logarithmic fits alone. Meaningful values for $z_{0}$ can be obtained from $(16)$ and are $z_{0} \approx 0.3,0.5,0.5$, and $0.5 \mathrm{~mm}$ based on the drag coefficients at pods $1-4$, respectively. According to these values, we chose the representative bottom roughness $z_{0}=0.5 \mathrm{~mm}$ for our modeling study.

\subsubsection{Bottom Stress Estimates from the $M_{2}$ Velocity Defect}

Vertical integration of the momentum equations gives an independent bottom stress estimate which can be compared to results from logarithmic fits. For the $M_{2}$ component, integrating (7) from bottom to surface yields

$$
\tau_{b}^{ \pm}(z)=-i\left(f \pm \omega_{M_{2}}\right) \int_{0}^{H} \mathbf{R}^{ \pm} d z-\left(z_{\infty}-z\right) \mathbf{P}^{ \pm}
$$

where $H$ is the height of the water column. With (8), expression (17) becomes a function of the tidal velocity defect

$$
\boldsymbol{\tau}_{b}^{ \pm}=-i\left(f \pm \omega_{M_{2}}\right) \int_{0}^{H}\left(\mathbf{R}^{ \pm}-\mathbf{R}_{\infty}^{ \pm}\right) d z
$$

Taking $\mathbf{R}^{ \pm}$and $\mathbf{R}_{\infty}^{ \pm}$from observation, the major and minor axes of the bottom stress ellipse amount to $\tau_{b_{M a j}}=1.96 \pm 0.48 \cdot 10^{-4} \mathrm{~m}^{2} \mathrm{~s}^{-2}$ and $\tau_{b_{M i n}}=-1.52 \pm$ $0.49 \cdot 10^{-4} \mathrm{~m}^{2} \mathrm{~s}^{-2}$, where uncertainties are the $95 \%$ confidence limits obtained from linear propagation of the standard error. For comparison, bottom stress was also computed from

$$
\left(\tau_{b}^{x}, \tau_{b}^{y}\right)=u_{*}^{2} \frac{\left(u_{b}, v_{b}\right)}{U_{b}}
$$

where $\left(u_{b}, v_{b}\right)$ and $U_{b}$ are the velocity components and magnitude averaged over BASS pods 1-3 (0.2-1.2 mab), respectively. Tidal decomposition of (19) gives $\tau_{b_{M a j}}=1.91 \pm 0.04 \cdot 10^{-4} \mathrm{~m}^{2} \mathrm{~s}^{-2}$ and $\tau_{b_{M i n}}=-0.95 \pm 0.04 \cdot 10^{-4} \mathrm{~m}^{2} \mathrm{~s}^{-2}$ within the error limits of the tidal fit. According to these values, friction velocities from logarithmic fits are within -12 to $+13 \%(-3$ to $-45 \%)$ of those based on the velocity defect method along the major (minor) axis of the near-bottom current ellipse. This 
comparison indicates uncertainties of the fits are mostly smaller than suggested by the $95 \%$ confidence limits shown in Fig.2.14.b.

Taking $\boldsymbol{\tau}^{ \pm}$from tidal decomposition of (19) and $\mathbf{P}^{ \pm}$as unknown, (17) can be solved for the pressure forcing. This gives $P_{M a j}=3.25 \pm 0.11 \cdot 10^{-5} \mathrm{~m} \mathrm{~s}^{-2}$ and $P_{\text {Min }}=0.11 \pm 0.11 \cdot 10^{-5} \mathrm{~m} \mathrm{~s}^{-2}$, with uncertainties given at the $95 \%$ confidence limit based on linear propagation of the standard error (Fig.2.12, solid). These results are in good agreement with estimates from (8), confirming the earlier conclusion that free-stream conditions are approached near the surface.

\subsection{Numerical Modeling}

Observations of the $M_{2}$ currents and bottom stress are compared to numerical results from two one-dimensional numerical circulation models: a two-layer eddy viscosity (2LK) model with linear-constant eddy viscosity distribution, and a continuous eddy viscosity model utilizing the Mellor-Yamada level 2.5 (MY2.5) turbulence closure scheme. Equations and parameterizations entering the $2 \mathrm{LK}$ and MY2.5 models are listed in Appendices B and C, respectively. Both models were forced with the $M_{2}$ pressure gradient computed from the vertically integrated momentum equations (Fig.2.12, solid). At the lower boundary, we specified the physical bottom roughness $z_{0}=0.5 \mathrm{~mm}$ based on measurements (see section 2.6.4).

The sensitivity of numerical solutions to weak stratification and wind mixing is examined using the MY 2.5 model. Model runs were performed for $N^{2}=0$ and a $N^{2}$ distribution representative of observations during the 26.7-day study period.

\subsubsection{Performance of the 2LK Model}

Results from the 2LK model show good agreement with the observed $M_{2}$ current distribution (Fig.2.17, solid). Predicted $R^{ \pm}$amplitudes are almost identical to observations in the lowest $20 \mathrm{~m}$ of the water column, and the veering of phase and inclination angles is within the error limits of the measurements. The predicted orientation of the surface tidal ellipse deviates less than $3^{\circ}$ from the observed values. According to numerical results, the clockwise component does not reach freestream conditions over the given water depth. The consequence is overprediction $(<$ $1 \mathrm{~cm} \mathrm{~s}^{-1}$ ) of $R^{-}$near the surface. Doubling the water depth yields $R^{-}$amplitudes 
that overshoot the expected free-stream values by $1-2 \mathrm{~cm} \mathrm{~s}^{-1}$ at $40-80 \mathrm{mab}$, in agreement with analytical predictions for constant $K$ (Ekman, 1905).

Model friction velocities are $1.2 \mathrm{~cm} \mathrm{~s}^{-1}$ on the tidal average, in close agreement with $\bar{u}_{*}=1.2 \mathrm{~cm} \mathrm{~s}^{-1}$ from best-fit logarithmic profiles to the BASS data. Bottom stress predictions along the major and minor axis of the near-bottom current ellipse are within $3 \%$ of the corresponding observational values. The sublayer evolves toward a steady state thickness of $5 \mathrm{~m}$, approximately equal to the observed height of the logarithmic layer (Fig.2.18.a).

\subsubsection{Performance of the MY2.5 Model}

The MY2.5 model without stratification shows fair agreement with the observations, but does not compare as well as the 2LK model (Fig.2.17, dashed). Results for the clockwise component show the largest discrepancies, with amplitudes that overpredict the measurements by as much as $3 \mathrm{~cm} \mathrm{~s}^{-1}$ below 20 mab, and phase veering that is smaller than observed. Underestimation of $\Delta \phi^{-}$leads to counterclockwise rotation of the major axis toward the bottom, as opposed to the observationally better supported clockwise-counterclockwise veering predicted by the $2 \mathrm{LK}$ model. Due to overprediction of near-bottom shear, the computed bottom stress exceeds the observations by $8 \%$ and $11 \%$ along the major and minor axis, respectively.

Weak stratification was added to the model by prescribing the time-mean density distribution (Fig.2.19). Prognostic representation of the density field was chosen because scaling arguments reveal vertical stratification is maintained by advective processes not included in our model (see Appendix C). Fig.2.20 (solid) shows weak stratification improves the model results in the lower water column. Compared to the case $N^{2}=0, R^{-}$amplitudes are reduced near the bottom, the phase veering $\Delta \phi^{-}$is more pronounced, and the rotation of the major axis indicates better agreement with the observations (Fig.2.17, dashed, and Fig.2.20, solid). The reduction of near-bottom shear results in smaller bottom stress magnitudes within $\pm 4 \%$ of the observations. In the upper water column, comparison to data is not as favorable. $R^{-}$amplitudes display a distinct peak near 45 mab, followed by a narrow $(\approx 10 \mathrm{~m})$ transition zone above which the profile is vertically constant. The explanation is that stratification limits the vertical extent of the BBL, dividing the water column into a free stream with no mixing and zero shear, and a turbulent, sheared region underneath. Since the clockwise boundary layer extends further 
into the stratified interior than the counterclockwise boundary layer, the effects of stratification are apparent for $\mathbf{R}^{-}$but not $\mathbf{R}^{+}$(Souza and Simpson, 1996). Eddy viscosities elucidate the mixing behavior of the model, showing significantly smaller $K$ for $N^{2}>0$ than for $N^{2}=0$ (Fig.2.18b,c). For stratified conditions, the model suggests vertical diffusion above 50 mab happens on molecular scales $\left(K=\nu=10^{-6} \mathrm{~m}^{2} \mathrm{~s}^{-2}\right.$ ), and is up to four orders of magnitudes larger in the BBL (Fig.2.18c):

The obvious discrepancy between model predictions and observations suggests wind mixing plays a role in determining the turbulence field. Wind measurements display largest variability at low frequencies ( $<$ cpd, Fig.2.11), and force vertically sheared currents that evolve on subtidal time scales. The applied surface momentum flux acts to transfer momentum downward, thus presenting a mechanism by which the surface and bottom turbulent layers can merge. Such behavior is supported by Richardson number estimates in the pycnocline (see section 2.4.3), showing $R i<R i_{c}$ more often if computations include the effects of subtidal current shear (Fig.2.9b,c).

Due to large wind variability in the subtidal frequency band, it is difficult to define a characteristic constant wind stress to be used in the model. Here, we added wind forcing representative of the time mean $\left(\tau_{w}=1.1 \cdot 10^{-4} \mathrm{~m}^{2} \mathrm{~s}^{-2}\right)$, and the time mean plus one standard deviation $\left(\tau_{w}=2.3 \cdot 10^{-4} \mathrm{~m}^{2} \mathrm{~s}^{-2}\right)$. Eddy viscosity profiles for $\tau_{w}=1.1 \cdot 10^{-4} \mathrm{~m}^{2} \mathrm{~s}^{-2}$ show the surface and bottom turbulent layers collide near 50 mab (Fig.2.18d). However, the overlap region is not wide enough to produce the amount of mixing necessary to change the velocity distribution compared to the case $\tau_{w}=0$ (not shown). On the other hand, increasing wind forcing to $\tau_{w}=2.3 \cdot 10^{-4} \mathrm{~m}^{2} \mathrm{~s}^{-2}$ clearly causes the bottom and surface turbulent layers to merge (Fig.2.18e). In response, the predicted peak of $R^{-}$almost completely disappears, giving rise to a smooth velocity profile in good agreement with the observations (Fig.2.20, dashed).

The results described above can be interpreted in at least two ways. First, merging of the surface and bottom boundary layers may occur intermittently during times of strong winds or extremely weak stratification. Alternatively, the MY2.5 model may overestimate the inhibiting effect of stratification on turbulent mixing. To evaluate which interpretation is more plausible, we examined the period $0000 \mathrm{UT}$ March 11 to 0000 UT March 26 (P2) immediately following the first investigation 
period (P1). Vertical stratification in the pycnocline was about four times larger, and wind stress magnitudes were about $30 \%$ smaller during P2 than P1 (not shown). Despite differences in vertical stratification, $M_{2}$ current amplitudes from tidal analysis were almost identical for both investigation periods (not shown). On the other hand, model predictions for P2 with realistic pressure and wind forcing (not shown) strongly resemble those for P1 with $\tau_{w}=0$ (Fig.2.20, dashed). Even for wind stress exceeding the time mean by three standard deviations, the model predicts little overlap between the surface and bottom boundary layers, and a mid-depth velocity maximum similar to Fig.2.20a (dashed) is distinctly visible. This behavior suggests the MY2.5 model underpredicts turbulent momentum transfer across the pycnocline.

\subsubsection{Discussion of the MY2.5 model}

In support of our results, Stacey et al. (submitted) found the MY2.5 model underpredicts the turbulent kinetic energy at and above the density interface in a shallow partially-stratified tidal channel in upper San Francisco Bay. On the other hand, Simpson et al. (1996) reported good agreement between measured and modeled dissipation rates at a $90-\mathrm{m}$ deep site in the Irish Sea, using a turbulence closure scheme similar to the MY2.5, i.e., their MY2.2b. The MY2.2b model solves the momentum and turbulent kinetic energy equations specified in the MY2.5 model, but utilizes the prognostic length scale $\ell=\kappa z\left(1-\frac{z}{H}\right)^{1 / 2}$ instead of the $q^{2} l$-equation (Appendix C). Applying Simpson et al.'s length scale parameterization to our case gives results almost identical to those from the MY2.5 closure, indicating differences in mixing length do not explain the under-estimation of turbulent momentum transfer in the pycnocline. A closer look at Simpson et al.'s results may reveal why our conclusions differ. Although dissipation profiles in the Irish Sea are qualitatively well predicted, the MY2.2b model underestimates the observed values by up to one order of magnitude in the strongly stratified upper third of the water column (Fig.2.8a,b in Simpson et al., 1996). According to Osborn (1980), the diapycnal diffusivity can be estimated from $\epsilon$ using

$$
K_{\rho}=0.2 \frac{\epsilon}{N^{2}}
$$


An order one under-prediction of $\epsilon$ thus implies an order one under-prediction of $K_{\rho}$. With $K_{M} \simeq K_{\rho}$, the eddy viscosity $K_{M}$ is similarly underestimated, shifting the balance in the momentum equations toward the time derivative and Coriolis terms. If the top of the TBL extends into the strongly stratified pycnocline, the order one under-estimation of $\epsilon$ may involve significant differences between modeled and observed velocities. Simpson et al.'s (1996) study does not include a model-data comparison of tidal current distributions, so that no inferences can be made concerning the realistic prediction of velocity profiles. With semidiurnal current amplitudes $U=0.3 \mathrm{~m} \mathrm{~s}^{-1}$ according to Simpson et al. (1996), tidal mixing at the $90-\mathrm{m}$ deep study site in the Irish Sea is weaker than on the $76-\mathrm{m}$ isobath on the southern flank of Georges Bank. This may imply the TBL in the Irish Sea is confined to the region well below the density interface, so that the ability of the model to correctly predict turbulent momentum transfer in the presence of stratification cannot be tested at the stratified site discussed in Simpson et al. (1996).

The questions about the MY2.5 model performance raised here will be discussed further in a subsequent paper (Part II), which describes observations and model results for strongly stratified conditions on the southern flank of Georges Bank.

\subsection{Summary and Conclusions}

We presented above observations of the tidal bottom boundary layer at a 76$\mathrm{m}$ deep study site on the southern flank of Georges Bank, a region dominated by the semidiurnal $M_{2}$ current constituent. Our analysis was carried out for the period February 11-March 11 representative of typical winter conditions. From our data, we inferred the vertical distribution of temperature, salinity, and density, obtained Richardson number estimates, computed bottom friction parameters such as bottom stress, and derived characteristic $M_{2}$ current profiles. The tidal velocity distribution and estimates of bottom stress were compared to predictions from two one-dimensional numerical models, i.e., a two-layer eddy viscosity model and the advanced MY2.5 model. Results from the observational and numerical analysis are as follows: 
- Density profiles reveal the presence of a nearly homogeneous bottom boundary layer located beneath a winter pycnocline $\left(N^{2} \approx 10^{-5} \mathrm{~s}^{-2}\right)$ and a surface mixed layer. Results from the Richardson number analysis show turbulent mixing is intense in the bottom and surface boundary layers, while it is limited at mid-depth in the region occupied by the pycnocline. Comparison of Richardson number estimates based on the vertical shear of the total (tidal and subtidal combined) and tidal currents indicates subtidal flow shear plays an important role in maintaining a reduced level of turbulence between the surface and bottom mixed layers. This behavior suggests nontidal processes such as wind mixing may transfer turbulent momentum across the pycnocline.

- Scaling arguments reveal the mechanisms sustaining weak winter stratification on Georges Bank are at least two-dimensional. Among such mechanisms are crossbank advection of the density field, and, in the strongly sheared TBL, tidal straining.

- The $M_{2}$ current profiles show a region of strong shear in the lower water column, also marked by increasing phase-lead and rotation of the velocity vector toward the bottom. This region constitutes the TBL, and coincides with the area of most intense mixing below the pycnocline. Decomposition of the tidal currents into rotary components indicates the presence of two distinct boundary layer thicknesses, in agreement with analytical predictions for tidal flow in the presence of bottom friction (Prandle, 1982a,b) and with earlier observations in shelf seas (Soulsby, 1983; Maas and van Haren, 1987). Our results indicate the observed TBL thickness is consistent with scaling arguments assuming a logarithmic layer near the bottom, similar to suggestions by Grant and Madsen (1986) for rectilinear nonrotational flow.

- Numerical modeling of the observed tidal current profiles is possible with a simple one-dimensional model assuming a two layer eddy viscosity structure with linear/constant distribution (the $2 \mathrm{LK}$ model). For the weakly stratified conditions examined here, the performance of the $2 \mathrm{LK}$ model is very good. Model predictions of current profiles and bottom stress agree well with data, and are at least as accurate as solutions from the more advanced Mellor Yamada level 2.5 (MY2.5) model. This supports earlier conclusions by Davies (1991), who found simple eddy viscos- 
ity parameterizations can compete with advanced higher order schemes if the flow conditions are homogeneous.

- Results from the MY2.5 model show the agreement between observational and numerical data depends on the specification of stratification and wind forcing. Taking $N^{2}=0$ with no surface wind, the model predicts intense tidal mixing with eddy viscosities that exceed those from the $2 \mathrm{LK}$ model. As a result; model solutions overestimate the near-bottom currents and bed shear stress by about $10 \%$, and do not predict sufficient veering of the velocity vector toward the bottom.

Introducing weak stratification representative of the observations limits the vertical extent of the turbulent TBL and improves model predictions in the TBL. At the same time, the model does not allow for turbulent momentum transfer across the pycnocline and separates the TBL from the free stream above it. The result is abrupt adjustment from the frictionally driven TBL to vertically uniform amplitudes in the free stream, causing large shear at the base of the pycnocline in disagreement with the observed smooth velocity distribution. Thus, we draw two inferences from the model solutions for $N^{2}>0$ : first, weak stratification representative of winter may affect the current distribution in the TBL by limiting vertical mixing compared to unstratified conditions. Second, processes other than tidal mixing may be responsible for turbulence production near the surface and in the pycnocline. Since high frequency internal waves were not observed during the weakly stratified conditions presented here, we exclude wave breaking as a possible source of mid-depth turbulence. This leaves wind-driven shear as a likely candidate for turbulence production in the upper water column.

- Adding wind forcing to the MY2.5 model introduces an additional source of turbulence whose effects superimpose on tidal mixing. Depending on the wind stress magnitude, the wind-driven circulation may penetrate sufficiently deep to transfer turbulent momentum from the upper layer across the winter pycnocline. Such behavior is supported by our Richardson number estimates, which suggest a limited level of turbulence is sustained in the pycnocline, although mixing is reduced compared to the bottom and surface boundary layers. Model solutions predict wind stress representative of the observed time mean is not sufficient to cause mixing at mid-depth. According to the model, turbulent momentum transfer across the 
pycnocline occurs if the applied wind stress exceeds the time-mean value by at least one standard deviation, or if vertical stratification is weaker than suggested by the temporal average. For large enough wind forcing, merging of the bottom and surface turbulent layers eliminates the separation between the lower and upper water column, resulting in a smooth current distribution in good agreement with the observations. These results clearly demonstrate possible impacts of wind mixing on the vertical structure of the tidal currents.

- The model-data comparison presented here does not provide convincing evidence for the ability of the MY2.5 model to correctly incorporate the effects of stratification. In particular, analysis of a later period representative of early spring evokes doubts about the model performance during stratified conditions. With $N^{2}$ between values characteristic of late winter $\left(\sim 10^{-5} \mathrm{~s}^{-2}\right)$ and summer $(\sim$ $\left.10^{-4}-10^{-3} \mathrm{~s}^{-2}\right)$, the model allows for little turbulence production at mid-depth, even for wind forcing that exceeds the observed time mean by three standard deviations. The predicted strong shear at the base of the pycnocline is in clear disagreement with our measurements, which suggest a smooth velocity profile. 


\section{Appendix A: Scaling of $\delta^{ \pm}$}

With the pressure forcing

$$
\mathbf{P}^{ \pm}=-i\left(f \pm \omega_{M_{2}}\right) \mathbf{R}_{\infty}^{ \pm},
$$

vertical integration of $(10)$ yields

$$
i\left(f \pm \omega_{M_{2}}\right) \int_{0}^{\delta^{ \pm}}\left(\mathbf{R}^{ \pm}-\mathbf{R}_{\infty}^{ \pm}\right) d z=-\tau_{b}^{ \pm}
$$

so that

$$
\delta^{ \pm}\left|f \pm \omega_{M_{2}}\right| \cdot\left|\frac{1}{\delta^{ \pm}} \int_{0}^{\delta^{ \pm}}\left(\mathbf{R}^{ \pm}-\mathbf{R}_{\infty}^{ \pm}\right) d z\right|=\left|\tau_{b}^{ \pm}\right| .
$$

Using the quadratic drag law

$$
\tau_{b}=c_{D} \cdot U_{b}^{2}
$$

to rewrite the bottom stress vector gives

$$
\left(\tau^{x}, \tau^{y}\right)_{b}=c_{D} \cdot U_{b}^{2} \cdot \frac{(u, v)_{b}}{U_{b}}
$$

where $U$ is current speed and the subscript $b$ refers to bottom. With

$$
\tau_{b}=u_{*}^{2}
$$

(A.5) becomes

$$
\left(\tau^{x}, \tau^{y}\right)_{b}=\sqrt{c_{D}} \cdot u_{*} \cdot(u, v)_{b}
$$

For a rotating $M_{2}$ tide, the friction velocity $u_{*}$ consists of a time-mean $\bar{u}_{*}$ and 
a time-varying $u_{*}^{2 \omega_{M_{2}}}$, where the superscript $2 \omega_{M_{2}}$ denotes the frequency of the variation. Writing $u_{*}=\bar{u}_{*}+u_{*}^{2 \omega_{M_{2}}}$, expression $\mathrm{A} .7$ becomes

$$
\left(\tau^{x}, \tau^{y}\right)_{b}=\sqrt{c_{D}} \cdot\left(\bar{u}_{*}+u_{*}^{2 \omega_{M_{2}}}\right) \cdot(u, v)_{b}
$$

In (A.7) only the product $\sqrt{c_{D}} \cdot \bar{u}_{*} \cdot(u, v)_{b}$ contributes to the tidal variation in bottom stress, while the term $\sqrt{c_{D}} \cdot u_{*}^{2 \omega_{M} M_{2}} \cdot(u, v)_{b}$ causes higher order fluctuations. The rotary components of the bottom stress vector can be expressed as

$$
\boldsymbol{\tau}_{b}^{ \pm}=\bar{u}_{*} \cdot \sqrt{c_{D}} \mathbf{R}_{b}^{ \pm}
$$

suggesting $\left|\sqrt{c_{D}} \cdot \mathbf{R}^{ \pm}\right|$represents an effective friction velocity $u_{*}^{ \pm}$acting upon $\mathbf{R}^{ \pm}$. Assuming the depth-averaged velocity defect is proportional to the effective friction velocity gives

$$
\left|\frac{1}{\delta^{ \pm}} \int_{0}^{\delta^{ \pm}}\left(\mathbf{R}^{ \pm}-\mathbf{R}_{\infty}^{ \pm}\right) d z\right| \propto\left|\sqrt{c_{D}} \mathbf{R}^{ \pm} \mathbf{b}\right|
$$

Substitution of (A.9)-(A.10) in (A.3) yields

$$
\delta^{ \pm} \propto \frac{\bar{u}_{*}}{\left|f \pm \omega_{M_{2}}\right|}
$$

in agreement with (13).

\section{Appendix B: The Two-Layer-K (2LK) Model}

The 2LK model is a one-dimensional finite-difference code that integrates the linear momentum equations (10) forward in time, using the parameterization $\left(\tau_{x}, \tau_{y}\right)=$ $K \cdot\left(\frac{\partial u}{\partial z}, \frac{\partial v}{\partial z}\right)$ to describe the stress vector. The resolution of the vertical grid is $\Delta z=0.5 \mathrm{~m}$. Initial conditions are the state of rest with $K=\nu$, where $\nu=10^{-6} \mathrm{~m}^{2} \mathrm{~s}^{-1}$ is the molecular viscosity of sea water. 
The boundary conditions at the surface $(z=H)$ and bottom $\left(z=z_{0}\right)$ are

$$
\begin{array}{cc}
\frac{\partial u}{\partial z}=\frac{\partial v}{\partial z}=0 & \text { at } z=H \\
K\left(\frac{\partial u}{\partial z}, \frac{\partial v}{\partial z}\right)=\left(\tau_{b}^{x}, \tau_{b}^{y}\right) & \text { at } z=z_{0}
\end{array}
$$

with $z_{0}=0.5 \mathrm{~mm}$ based on results from data analysis. Bottom stress is computed from the quadratic drag law

$$
\left(\tau_{b}^{x}, \tau_{b}^{y}\right)=c_{D} \cdot \sqrt{u^{2}+v^{2}}(u, v)
$$

where

$$
c_{D}=\left[\frac{\kappa}{\ln \left(\frac{z_{b}}{z_{0}}\right)}\right]^{2},
$$

and $z_{b}=0.25 \mathrm{~m}$ is the height of the lowest grid point. After completion of each tidal cycle, $K$ is computed according to

$$
K=\left\{\begin{array}{lll}
\kappa & \bar{u}_{*} z & \text { for } z \leq l \\
\kappa & \bar{u}_{*} l & \text { for } z>l
\end{array},\right.
$$

where the overbar denotes time-average over one tidal cycle, and $\ell$ is a sublayer height.

Meaningful parameterizations of the sublayer height specify $\ell=a \cdot \delta$, where $\delta$ is a vertical scale for the total boundary layer thickness, and $a$ is an empirical constant (Trowbridge and Madsen, 1984). For steady planetary and rectilinear oscillating flows, representative scale expressions are $\delta \simeq \frac{\kappa u_{*}}{f}$ and $\delta \simeq \frac{\kappa \bar{u}_{*}}{\omega}$, respectively (Grant and Madsen, 1986). In the present case of a rotating tidal current with $R^{-}>4 R^{+}$, the clockwise boundary layer dominates the current distribution, so that $\delta=\frac{\kappa \bar{u}_{*}}{\omega_{M_{2}}-f}$ is a characteristic boundary layer scale. The empirical constant $a$ is not well known, and its specification is somewhat arbitrary. Trowbridge and Madsen (1984) suggest $a=\frac{1}{6}$ for non-rotational rectilinear flows, because model results using this value are in good agreement with laboratory experiments by Jonsson and Carlsen (1976). Similar scaling was applied by Beardsley et al. (1995) to investigate 
rectilinear tidal currents on the Amazon Shelf. For Georges Bank, $a=\frac{1}{6}$ overpredicts the observed bottom stress and current magnitudes in the bottom boundary layer. Numerical experiments show model results agree well with observations if $a=\frac{1}{20}$, so that

$$
l=\frac{1}{20} \cdot \frac{\kappa \bar{u}_{*}}{\omega_{M_{2}}-f}
$$

is used to determine the sublayer height after each tidal cycle. With $\bar{u}_{*}=1.2 \mathrm{~cm}$ $\mathrm{s}^{-1}$ according to data and model results (see sections 2.6.3 and 2.7.1), (B.5) yields $\ell \approx 5 \mathrm{~m}$ in close agreement with the observed logarithmic layer height (see section 2.6.1).

\section{Appendix C: The Mellor-Yamada Level 2.5 (MY 2.5) Model}

The MY2.5 turbulence closure model used here is a one-dimensional version of the Blumberg-Mellor hydrodynamic circulation model (Blumberg and Mellor, 1983, 1987), modified to include mixing length scale limitation by stable stratification (Galperin et al., 1988). Details of the turbulence closure are given in Mellor and Yamada (1974, 1982). Vertical grid, bottom roughness, and initial conditions are the same as for the 2LK model (Appendix B).

Model runs with $N^{2}>0$ require either the choice of initial conditions such that model solutions evolve toward the observed density distribution, or the a priori specification of density both in time and space. We chose the second approach, since physical processes sustaining vertical stratification on Georges Bank are more than one-dimensional. This can be shown from scaling as follows. Assuming along-bank density gradients are small, the density equation may be written as

$$
\frac{\partial \rho}{\partial t}+u \frac{\partial \rho}{\partial x}=\frac{\partial}{\partial z}\left(K_{\rho} \cdot \frac{\partial \rho}{\partial z}\right)
$$

where $K_{\rho}$ is the diapycnal diffusivity. Taking the vertical derivative of (C.1) gives

$$
\frac{\partial N^{2}}{\partial t}+u \frac{\partial N^{2}}{\partial x}-\frac{g}{\rho} \frac{\partial u}{\partial z} \frac{\partial \rho}{\partial x}=\frac{\partial^{2}}{\partial z^{2}}\left(K_{\rho} N^{2}\right)
$$


The second term in (C.2) describes the cross-bank advection of buoyancy, i.e., onbank transport of more stratified water during flood, and off-bank transport of less stratified water during ebb (see section 2.4.3). The third term accounts for the effects of tidal straining due to tidal current shear. Modeling of $N^{2}$ with a one-dimensional model is possible only if horizontal advection and tidal straining are negligible compared to the time derivative and vertical diffusion terms. Characteristic variations of $N^{2}$ during a tidal cycle are $\left(\Delta N^{2}\right)^{M_{2}}=0.4 \cdot 10^{-5}\left(0.2 \cdot 10^{-5}\right) \mathrm{s}^{-3}$ in the pycnocline (BBL) (Fig.2.9a), so that the first term in C.2 scales

$$
\frac{\partial N^{2}}{\partial t} \sim \omega_{M_{2}} \cdot\left(\Delta N^{2}\right)^{M_{2}} \sim 5.6(2.8) \cdot 10^{-10} \mathrm{~s}^{-3} .
$$

Taking the tidal excursion $\ell_{M_{2}}=\frac{2 U}{\omega_{M_{2}}}=6 \mathrm{~km}$ as a representative horizontal scale, where $U=0.4 \mathrm{~m} \mathrm{~s}^{-1}$ is the cross-bank velocity amplitude (Fig.2.10d), gives for the second term

$$
u \frac{\partial N^{2}}{\partial x} \sim U \cdot \frac{\left(\Delta N^{2}\right)^{M_{2}}}{\ell_{M_{2}}} \sim 2.7(1.3) \cdot 10^{-10} \mathrm{~s}^{-3} .
$$

Tidal straining has no importance in the pycnocline where $M_{2}$ current shear is very small, but may be significant in the lowest $30 \mathrm{~m}$ of the water column (Fig.2.10). With typical density variations $\Delta\left(\sigma_{\theta}\right)^{M_{2}}=0.01$ between ebb and flood (Fig.2.8d), the straining term is about

$$
\frac{g}{\rho} \frac{\partial u}{\partial z} \frac{\partial \rho}{\partial x} \sim \frac{g}{\rho} \frac{U}{\Delta z} \frac{\Delta\left(\sigma_{\theta}\right)^{M_{2}}}{\ell_{M_{2}}} \sim 0.0(2.2) \cdot 10^{-10} \mathrm{~s}^{-3} .
$$

in the pycnocline (BBL), where $\Delta z=30 \mathrm{~m}$ corresponds to the approximate extent of the strongly sheared region above the bottom.

Scaling of the right hand term requires a rough estimate of $K_{\rho}$. According to the MY2.5 model, $K_{\rho} \simeq K$, so that a representative value in the pycnocline (BBL) is $K_{\rho} \simeq 0.006(0.02) \mathrm{m}^{2} \mathrm{~s}^{-2}$ (Fig.2.18d). With typical values of $N^{2}=$ $10^{-5}\left(2 \cdot 10^{-6}\right) \mathrm{s}^{-2}$ (Fig.2.19) and the vertical scale $\Delta z=30 \mathrm{~m}$ describing the thickness of the sheared lower water column and winter pycnocline (Figs.10), the right hand term becomes 


$$
\frac{\partial^{2}}{\partial z^{2}}\left(K_{\rho} \cdot N^{2}\right) \sim \frac{K_{\rho} \cdot N^{2}}{(\Delta z)^{2}} \sim 0.6(0.4) \cdot 10^{-10} \mathrm{~s}^{-3}
$$

According to (C.3)-(C.7), cross-bank advection of buoyancy is of similar magnitude as the time rate of change, and larger than the vertical diffusion term. In the BBL, tidal straining enters as an additional factor setting vertical stratification. Based on these results, it is not possible to predict the buoyancy field with a one-dimensional numerical model.

Acknowledgments: We thank Sandy Williams and his co-workers for collecting and making the BASS tripod data available, Janet Fredericks, Jim Churchill and Tom Gross for their assistance with BASS data processing, Steve Lentz, Bob Weller, Jim Manning and their co-workers for obtaining the moored meteorological and oceanographic data at ST1 and ST2 and making the data available, Neil Oakey and Dave Hebert and their co-workers for collecting the microstructure data at ST1 and making the data available, Dick Limeburner for determining conductivity sensor uncertainties, Nancy Brink and Nan Galbraith for processing the moored data, Carol Alessi for her help with managing the data set, and Anne-Marie Michael for typing large parts of this paper. Julio Candela provided the version of the M.G.G. Foreman tidal analysis code used in this study. The MY $2.51-\mathrm{D}$ numerical circulation model was provided by Stephen Monismith and is based on the Blumberg-Mellor 3-D hydrodynamic circulation model. We especially want to thank John Trowbridge and Steve Lentz for many helpful discussions and advice about all aspects of this study. This work was supported by the National Science Foundation under grants OCE 93-13671 and OCE 96-32357 as part of the U.S. GLOBEC/Georges Bank Program. 


\section{References}

Beardsley, R. C., J. Candela, R. Limeburner, W. R. Geyer, S. J. Lentz, B. M. Castro, D. Cacchione, and N. Carneiro, The $\mathrm{M}_{2}$ tide on the Amazon shelf. J. Geophys. Res., 100, 2283-2319, 1995.

Blumberg, A. F., and G. L. Mellor, A description of a three-dimensional coastal ocean circulation model. Coastal and Estuarine Science, 4, 1-16, 1987.

Brown, W. S., A comparison of Georges Bank, Gulf of Maine and New England Shelf tidal dynamics, J. Physical Oceanogr., 14, 145-167, 1984.

Butman, B., M. Noble, D. C. Chapman and R. C. Beardsley, An upper bound for the tidally rectified current at one location on the southern flank of Georges Bank. J. Physical Oceanogr., 11, 1452-1460, 1983.

Butman, B., J. W. Loder, and R. C. Beardsley, The seasonal mean circulation: Observation and theory. In: Georges Bank, R. H. Backus, editor, The MIT Press, pp. 125-138, 1987.

Chen, C., Variability of currents in Great South Channel and over Georges Bank: Observation and Modeling. Ph.D. Thesis, Joint Program in Oceanography/ Applied Ocean Science and Engineering, Woods Hole Oceanographic Institution, Woods Hole, Mass, Massachusetts Institute of Technology, Cambridge, Mass, 1992.

Chapman, D. C. and R. C. Beardsley, On the origin of shelf water in the Middle Atlantic Bight. J. Physical Oceanogr., 19, 384-390, 1989.

Clauser, F. H., The turbulent boundary layer. Advances in Applied Mechanics, 4, $1-51,1956$.

Davies, A. M., and J. E. Jones, Application of a three-dimensional turbulence energy model to the determination of tidal currents on the northwest European shelf. J. Geophys. Res., 95, 18,143-18,162, 1990.

Davies, A. M., On using turbulence energy models to develop spectral viscosity models. Continental Shelf Res., 11, 1,313-1,353, 1991. 
Davies, A. M., and J. N. Aldridge, A numerical model study of parameters influencing tidal currents in the Irish Sea. J. Geophys. Res., 98, 7,049-7.067, 1993.

Davies, A. M., and J. Xing, An inter-comparison and validation of a range of turbulence closure schemes used in three dimensional tidal models. Quantitative Skill Assessment for Coastal Ocean Models,, D. R. Lynch and A. M. Davies, editors, In: Coastal and Estuarine Studies, American Geophysical Union, 47, 71-95, 1995.

Davies, A. M., On the importance of time varying eddy viscosity in generating higher tidal harmonics. J. Geophys. Res., 95, 20,287-20,312, 1990.

Ekman, V. W., On the influence of the earth's rotation on ocean-currents. Ark. Mat., Astron. Ef Fys., 2, 55, 1905.

Flagg, C. N., Hydrographic structure and variability. In: Georges Bank, R. H. Backus, editor, The MIT Press, 108-124, 1987.

Foreman, M. G. G., Manual for tidal currents analysis and prediction, Pacific Marine Science Report 87-6, Institute of Ocean Science, Patricia Bay, Sidney, B.C, 1978.

Galperin, B., Kantha, L. H., Hassid, S. and A. Rosati, A quasi-equilibrium turbulent energy model for geophysical flows. J. Atmos. Sci., 45, 55-62, 1988.

Garrett, C., 1972: Tidal resonance in the Bay of Fundy and Gulf of Maine. Nature, 238, 441-443, 1979.

Grant, W. D., and O. S. Madsen, Combined wave and current interaction with a rough bottom. J. Geophys. Res., 84, 1,797-1,808, 1979.

Grant, W. D., and O. S. Madsen, Movable bed roughness in unsteady oscillatory flow. J. Geophys. Res., 87, 469-481, 1982.

Grant, W. D., A. J. Williams III, and S. M. Glenn, Bottom stress estimates and their prediction on the Northern California Continental Shelf during CODE1: The importance of wave-current interaction. J. Physical Oceanogr., 14, 506-525, 1984. 
Grant, W. D., and O. S. Madsen, The continental-shelf bottom boundary layer. Annual Rev. Fluid Mech., 18, 265-305, 1986.

Gregg, M.C., D. P. Winkel, and T. B. Sanford, Varieties of fully resolved spectra of vertical shear. J. Physical Oceanogr.,23, 124-141, 1993.

Gross, T. F., and A. R. M. Nowell, Mean flow and turbulence scaling in a tidal boundary layer. Continental Shelf Res., 2, 109-206, 1983.

Hopkins, T. S., and N. Garfield III, Physical Origins of Georges Bank water. J. Marine Res., 37, 103-139, 1981.

Jonsson, I. G., and N. A. Carlsen, Experimental and theoretical investigations in an oscillatory turbulent boundary layer. J. Hydraul. Res., 14, 45-60, 1976.

Kagan, B. A., On the structure of the tidal flow in the sea. Izv. Atmos. and Oceanic Phys., 2, 575-581, 1966 (English translation).

Kundu P. K., In: Fluid Mechanics, Academic Press Inc., San Diego, California, 384-385, 1990.

Large, W. G., and S. Pond, Open ocean flux measurements in moderate to strong winds, J. Physical Oceanogr., 11, 324-336, 1981.

Lavelle, J. W., and H. O. Mofjeld, Effects of time-varying viscosity on oscillatory turbulent channel flow. J. Geophys. Res., 88, 7,607-7,616, 1983.

Lentz, S. J., and B. Butman, Comparison of BASS and VACM current measurements during STRESS. J. Atmos. Oceanic Techn., 12, 1328-1337, 1995.

Loder, J. W., Topographic rectification of tidal currents on the sides of Georges Bank. J. Physical Oceanogr., 10, 1399-1416, 1980.

Loder, J. W., and D. G. Wright, Tidal rectification and front circulation on the sides of Georges Bank, J. Marine Res., 43, 581-604, 1985.

Maas, L. R. M., and J. J. M. van Haren, Observations on the vertical structure of tidal and inertial currents in the central North Sea. J. Marine Res., 45, $293-318,1987$. 
Mellor, G. L., and T. Yamada, A hierarchy of turbulence closure models for planetary boundary layers. J. Atmos. Sc., 33, 1791-1896, 1974.

Mellor, G. L., and T. Yamada, Development of a turbulence closure model for geophysical fluid problems. Rev. Geophys. Space Physics, 20, 851-875, 1982.

Mofjeld, H. O., Effects of vertical viscosity on Kelvin waves. J. Physical Oceanogr., 10, 1,039-1,050, 1980.

Naimie, C. E., On the modeling of the seasonal variation in the three-dimensional circulation near Georges Bank. Ph. D. Thesis, Thayer School of Engineering, Dartmouth College, Hanover, New Hampshire, 1995.

Osborn, T. R., Estimates of the local rate of vertical diffusion from dissipation measurements. J. Physical Oceanogr., 10, 83-89, 1980.

Ozmidov, R. V., On the turbulent exchange in a stably stratified ocean. Izv. Acad. Sci. USSR, Atmos. Ocean. Phys., (Eng. Transl.), 8, 493-497, 1965.

Prandle, D., The vertical structure of tidal currents. Geophys. Astrophys. Fl. Dynamics, 22, 29-49, 1982a.

Prandle, D., The vertical structure of tidal currents and other oscillatory flows. Continental Shelf Res., 1 (2), 191-207, 1982b.

Schlichting, H., Boundary Layer theory, 4th ed., McGraw-Hill, New York, 647 pp., 1968.

Simpson, J. H., and J. Sharples, Does the Earth's rotation influence the location of the shelf sea fronts? J. Geophys. Res., 99, 3,315-3,319, 1994.

Simpson, J. H., W. Crawford, T. P. Rippeth, A. R. Campbell and J.V.S. Cheok, The vertical structure of turbulent dissipation in shelf seas. J. Physical Oceanogr., 26, 1579-1590, 1996.

Soulsby, R. L., and K. R. Dyer, The form of the near-bed velocity profile in a tidally accelerating flow. J. Geophys. Res., 86, 8,067-8,074, 1981. 
Soulsby, R. L., The bottom boundary layer of shelf seas. Physical Oceanogr. of Coastal and Shelf Seas, B. Johns, editor, Elsevier, Amsterdam, pp. 189-266, 1983.

Souza, A. J., and J. H. Simpson, The modification of tidal ellipses by stratification in the Rhine ROFI. Continental Shelf Res., 16, 997-1007, 1996.

Stacey, M. T., S. G. Monismith, and J. R. Bureau, Observations of turbulence in a partially stratified estuary. Submitted to J. Physical Oceanogr..

Sverdrup, H. U., Dynamics of tides on the north Siberian shelf. Geofys. Publ. Norske Videns Akad., 4, Oslo, Norway, 1927.

Tee, K. T., Depth-dependent studies of tidally induced residual current on the sides of Georges Bank, J. Physical Oceanogr., 15, 1818-1846, 1985.

Tennekes, H., The logarithmic wind profile. J. Atmos. Sc., 30, 234-238, 1973.

Trowbridge, J., and O. S. Madsen, Turbulent wave boundary layers 1. Model formulation and first-order solution. J. Geophys. Res., 89, 7989-7997, 1984.

Visser, A. W., A. J. Souza, K. Hesner, and J. H. Simpson, The effect of stratification on tidal current profiles in a region of freshwater influence. Oceanologica acta, 17, 369-382, 1994.

Werner, S. R., The vertical structure of the bottom boundary layer on the southern flank of Georges Bank during late winter. M.S. Thesis, Joint Program in Oceanography/ Applied Ocean Science and Engineering, Woods Hole Oceanographic Institution, Woods Hole, Mass, Massachusetts Institute of Technology, Cambridge, Mass, 1996.

Williams, A. J., 3rd, J. S. Tochko, R.L. Koehler, W. D. Grant, T. F. Gross, and C.V.R. Dunn, Measurement of turbulence in the oceanic bottom boundary layer with an acoustic current meter array. J. Atmos. Oceanic Techn., 4, 312-327, 1987. 


\begin{tabular}{|c|c|}
\hline $\begin{array}{c}\text { Tidal } \\
\text { Constituent }\end{array}$ & $\begin{array}{c}\text { Percent of Total } \\
\text { Kinetic Energy }\end{array}$ \\
\hline $\mathrm{S}_{2}(12.00 \mathrm{hrs})$ & 3.1 \\
$\mathrm{M}_{2}(12.42 \mathrm{hrs})$ & 85.3 \\
$\mathrm{~N}_{2}(12.66 \mathrm{hrs})$ & 2.3 \\
$\mathrm{~K}_{1}(23.93 \mathrm{hrs})$ & 0.6 \\
$\mathrm{O}_{1}(25.82 \mathrm{hrs})$ & 0.2 \\
High frequency $(<12 \mathrm{hrs})$ & 2.4 \\
Subtidal $(>33 \mathrm{hrs})$ & 5.8 \\
\hline
\end{tabular}

Table 2.1: Kinetic energy of the depth-averaged diurnal and semidiurnal tidal constituents, highfrequency components (time scales $<33 \mathrm{hrs}$ ), and subtidal flow (time scales $>33 \mathrm{hrs}$ ) in percent of total kinetic energy at ST1. 




Fig.2.1: Bathymetry (in meters) of Georges Bank and the Gulf of Maine. 


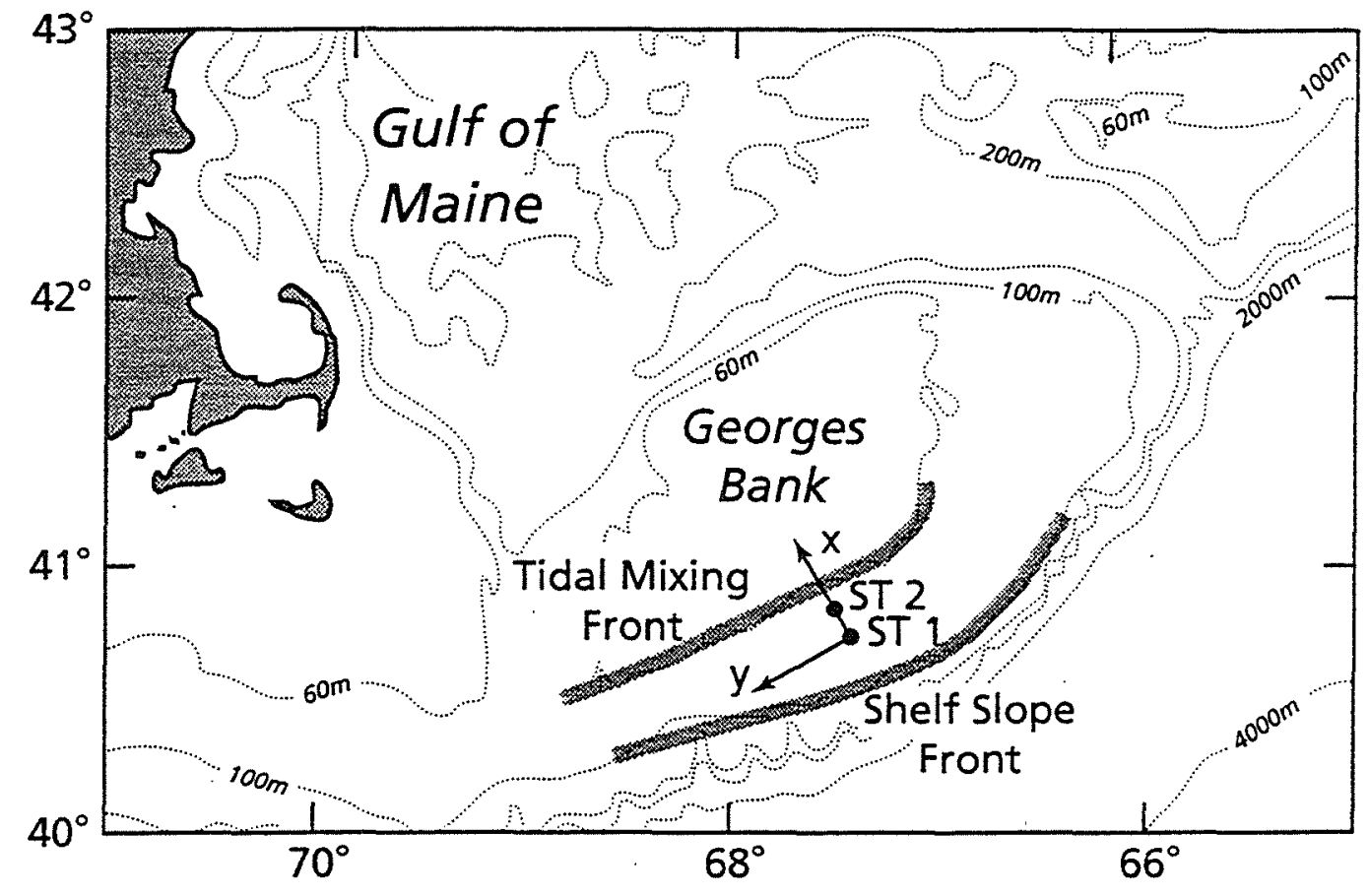

Fig.2.2: Bathymetry (in meters) of Georges Bank and adjacent region, approximate location of the Tidal Mixing Front (TMF), Shelf-Slope Front (SSF), and GLOBEC Stratification Study mooring sites ST1 and ST2. The $+x$ direction is on-bank $\left(330^{\circ} \mathrm{T}\right)$. 




Fig.2.3: Cross section of Georges Bank, showing the location of Georges Bank Water (GBW), the Tidal Mixing Front (TMF), and the Shelf-Slope Front (SSF). 




Fig.2.4: Schematic of the GLOBEC moored array at ST1 and ST2. 




Fig.2.5: Schematic of the GLOBEC BASS tripod. 




Fig.2.6: Temperature difference $\Delta T$ between BASS thermistors 8 and 2 at heights 5.7 and $0.6 \mathrm{mab}$. 
(a)



(c)



(b)



(d)

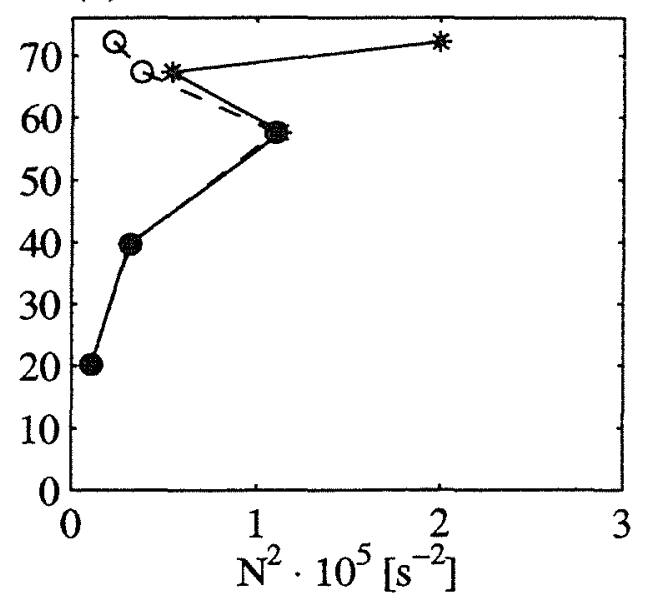

Fig.2.7: Profiles of time-mean (a) temperature $\mathrm{T}$, (b) salinity $\mathrm{S}$, (c) potential density $\sigma_{\theta}$, and (d) buoyancy frequency squared $N^{2}$ denoted by asterisks for February 11-March 11. Circles correspond to averages after isolated events of low-salinity water intrusions have been removed, leaving approximately $89 \%$ of the investigation period. 
Fig.2.8: (a) Schematic of the tidal ellipse, (b) temperature T, (c) salinity $S$, and (d) potential density $\sigma_{\theta}$ as a function of the depth-dependent tidal flow direction $\alpha$ divided in bins of $30^{\circ}$ width. Current measurements were interpolated linearly in the vertical to obtain estimates of the tidal flow direction at the SeaCAT heights, thereby accounting for the phase-lead of the near-bottom velocities with respect to the surface. Computations are based on half-hourly-averaged SeaCAT data, with error bars denoting the standard error at the $95 \%$ confidence level. The flow direction is measured clockwise from the $+x$ direction, such that flood (ebb) currents correspond to $\alpha=0^{\circ}\left(180^{\circ}\right)$. 




(b)


(c)


(d)




(b)


Fig.2.9: (a) Buoyancy frequency squared $N^{2}$, (b) percentage of events $R i<0.25$ and (c) percentage of events $R i<0.5$ as a function of the depth-dependent tidal flow direction $\alpha$ as described in Fig.2.8. Results are from half-hourly-averaged data. $R i$-estimates are based on (circles) total and (diamonds) high-passed (temporal variations $<33 \mathrm{hrs)}$ current shear. Error bars in (a) denote the standard error at the $95 \%$ confidence level. Error limits in (b) and (c) were obtained from Monte Carlo simulation (see text). 
(a)



(d)



(b)



(e)



(c)



(f)



Fig.2.10: Profile of the $M_{2}$ current ellipse parameters: (a) magnitudes $R^{+}$(smaller values) and $R^{-}$(larger values), veering angles (b) $\Delta \phi^{+}$and (c) $\Delta \phi^{-}$, (d) amplitudes $U_{M a j}$ and $U_{M i n}$, (e) rotation of the major axis $\Delta \theta$ (positive counterclockwise), and (f) phase angle $\Delta \phi$ (phaselead negative). Error bars denote the standard error of the tidal fit at the $95 \%$ confidence level, or, for $\Delta \phi^{ \pm}$and $\Delta \theta$, the compass uncertainty (depending on which is larger). 

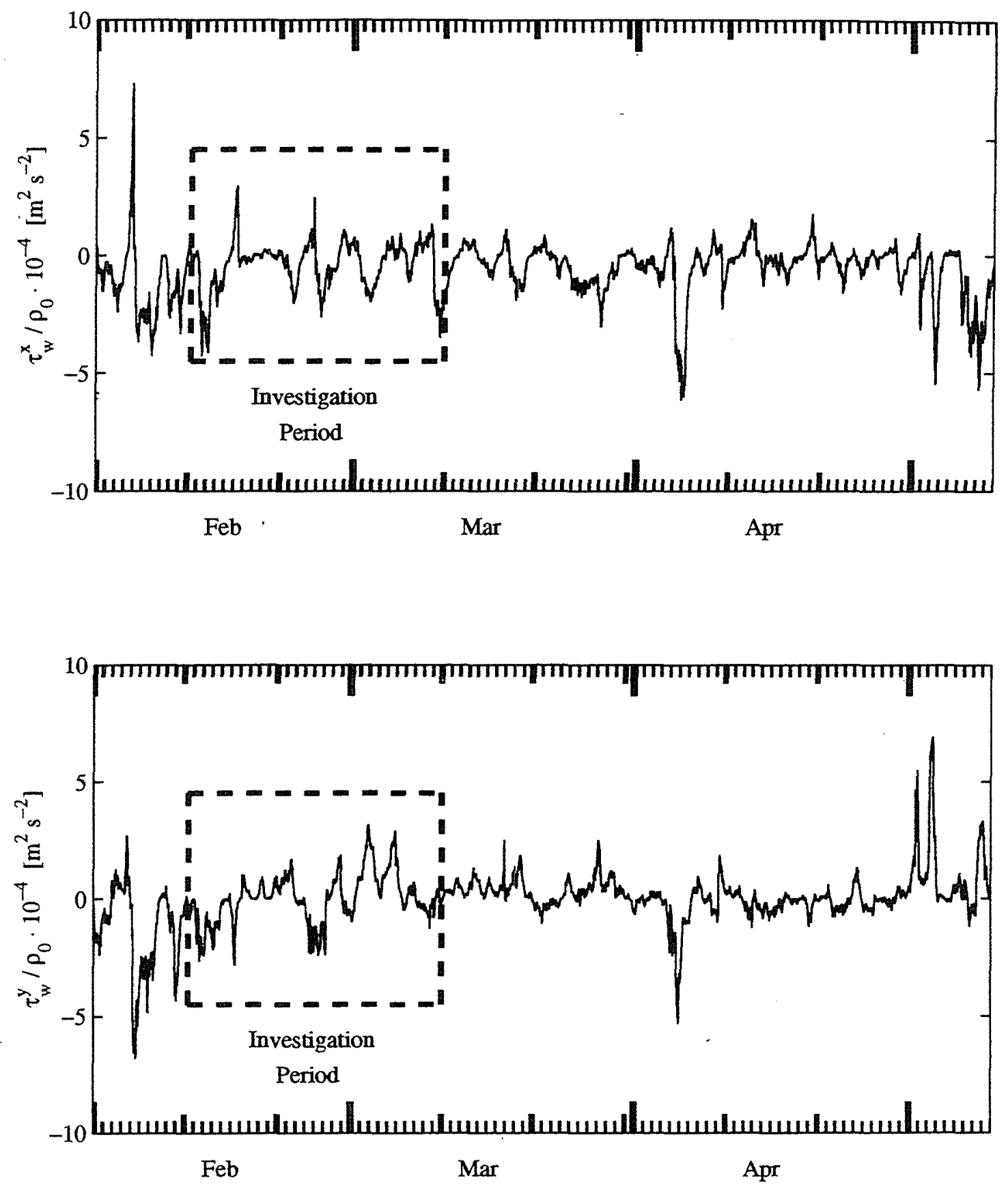

Fig.2.11: Time series of wind stress computed using the Large and Pond neutral stability algorithm (Large and Pond, 1981). 




Fig.2.12: Pressure forcing computed from (dashed) (8) and (solid) (17). 

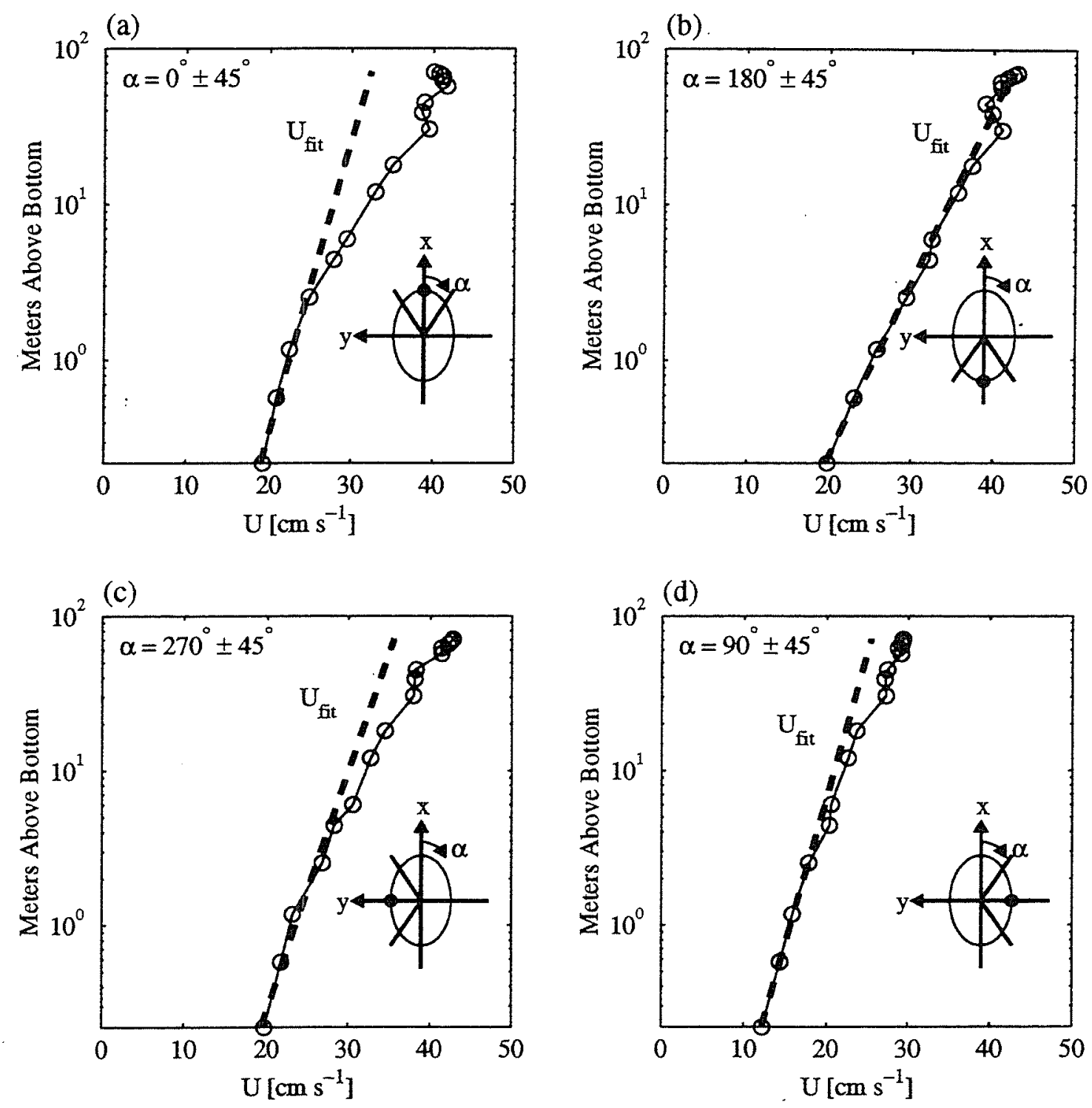

Fig.2.13: (Circles) profiles of averaged current speeds $U$ with near-bottom flow directions less than $\pm 45^{\circ}$ from the (a,b) cross-bank and (c,d) along-bank direction. The dashed line $U_{f i t}$ represents results extrapolated from least-squares logarithmic fits to measurements at the lowest four BASS current meters. 



Fig.2.14: (a) Time series of estimated friction velocity $u_{*}$, and (b) diagram of $\frac{\Delta u_{*}^{95}}{u_{*}}$ versus $u_{*}$. In (a), missing values $u_{*}$ are filled in by linear interpolation. 




Fig.2.15: Estimated friction velocity $u_{*}$ versus measured speed $U$, at $1.18 \mathrm{mab}$ (BASS pod 3). The solid line depicts the slope $\sqrt{c_{D}} \cdot U$ with $c_{D}$ obtained from least squares fits of $c_{D} U^{2}$ to $u_{*}^{2}$. Dashed lines give the $95 \%$ confidence limits of the fitted slope. 
(a)



(b)



Fig.2.16: Histograms of (a) bottom roughness $\log _{10}\left(z_{0}[\mathrm{~mm}]\right)$ and (b) $\frac{\Delta z_{0}^{95}}{z_{0}}$. 
(a)



(d)

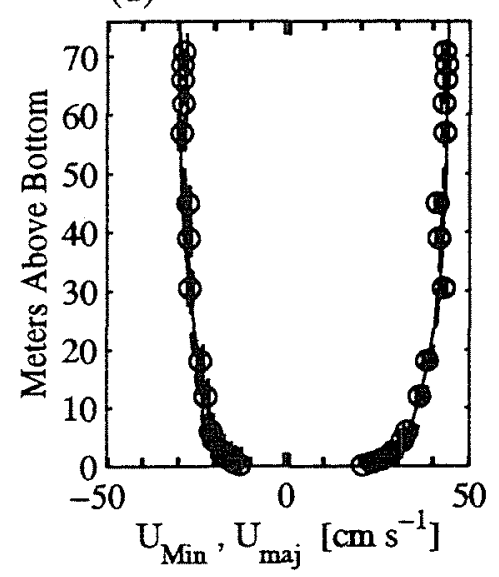

(b)



(e)



(c)



(f)



Fig.2.17: Tidal velocity profiles from the (solid) 2LK model with tidal forcing, and (dashed) MY2.5 model with tidal forcing and no stratification. Circles and error bars are from data as in Fig.2.10. 
(a)



(d)

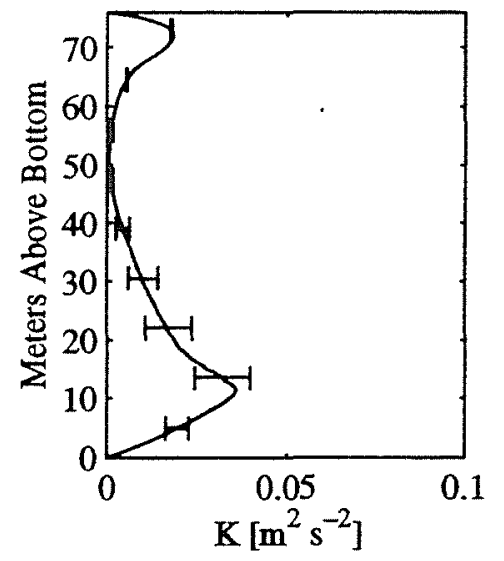

(b)



(e)

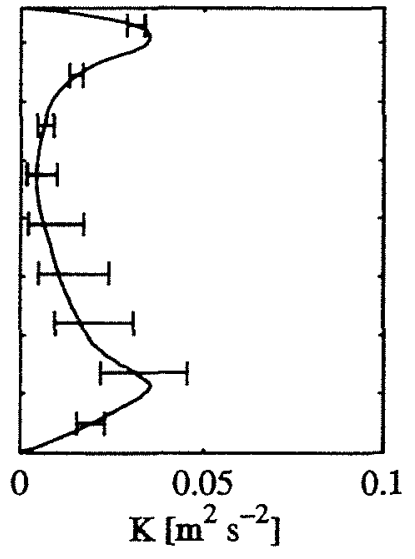

(c)

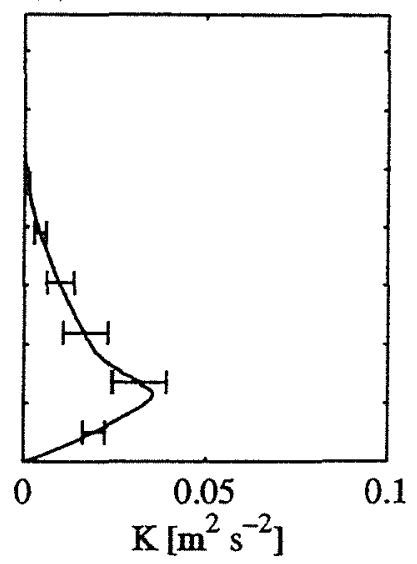

Fig.2.18: Eddy viscosities from the (a) 2LK model with tidal forcing , (b) MY2.5 model with tidal forcing and no stratification, (c) MY2.5 model with tidal forcing and vertical stratification according to Fig.2.19, (d) MY2.5 model as in (c) with additional wind forcing $\tau_{w}=1.1 \cdot 10^{-4} \mathrm{~m}^{2} \mathrm{~s}^{-2}$, and (e) MY2.5 model as in (c) with additional wind forcing $\tau_{w}=2.3 \cdot 10^{-4} \mathrm{~m}^{2} \mathrm{~s}^{-2}$. Horizontal bars in (b)-(e) represent variations during a tidal cycle. 


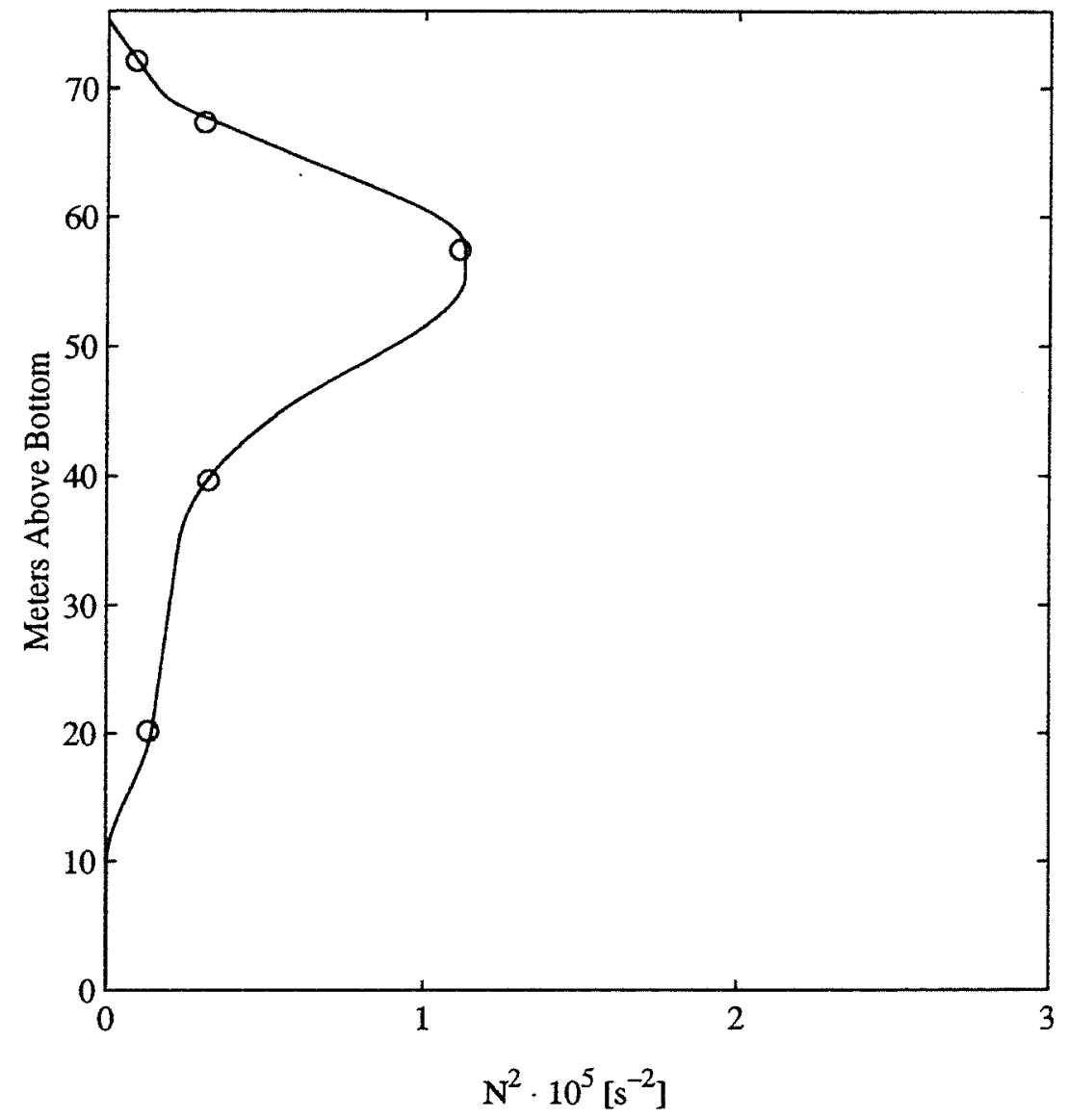

Fig.2.19: Vertical stratification according to (circles) data (see also dashed line in Fig.2.7d), and (solid line) as specified in the MY2.5 model. 
(a)



(d)



(b)

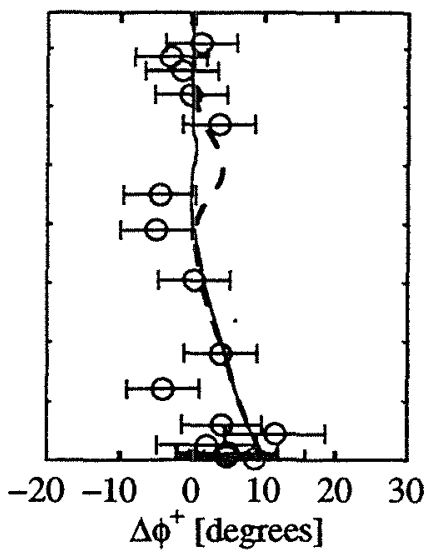

(e)



(c)

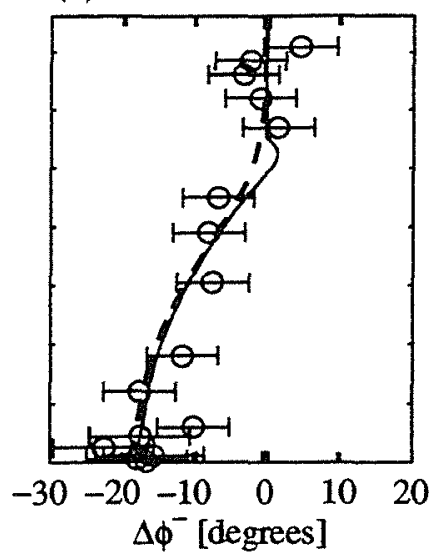

(f)



Fig.2.20: Tidal velocity profiles from the (solid) MY2.5 model with tidal forcing and stratification according to Fig.2.19, and (dashed) MY2.5 model with tidal forcing, stratification, and wind forcing $\tau_{w}=2.3 \cdot 10^{-4} \mathrm{~m}^{2} \mathrm{~s}^{-2}$. Circles and error bars are from data as in Fig.2.10. 


\section{Chapter 3}

\section{Observations and Modeling of the Tidal Bottom Boundary Layer, Southern Flank of Georges Bank. Part II: Strongly Stratified Conditions.}

\section{Preface}

Similar to Chapter 2, Chapter 3 represents the partially revised version of an earlier manuscript submitted for publication by Werner and Beardsley. 


\begin{abstract}
The vertical structure of the $M_{2}$ tidal boundary layer on the southern flank of Georges Bank is investigated under strongly stratified conditions representative of spring and summer. Current profiles, bottom stress estimates, and vertical stratification are inferred from moored acoustic current meter, VMCM, temperature and conductivity measurements made on the $76-\mathrm{m}$ isobath at $40^{\circ} 51.8^{\prime} \mathrm{N}, 67^{\circ} 33.5^{\prime} \mathrm{W}$. Observations are presented for a Shelf-Slope front intrusion in May 1995, and for stratified conditions representative of summer (July/August, 1995). Comparisons are drawn to nearly homogeneous winter conditions at the same location (Werner and Beardsley, submitted).
\end{abstract}

The $M_{2}$ constituent carries more than $85 \%$ of the kinetic energy at the moored array location and is responsible for a large part of the turbulence production at the lower boundary. Subtidal (time scales $>33 \mathrm{hrs}$ ) current shear contributes to turbulence production in the bottom boundary layer (BBL) when the tidal flow is roughly aligned with the mean along-bank circulation. Salinity, temperature and buoyancy frequencies squared display strong variations over the course of a tidal cycle, reflecting on-and off-bank advection of the cross-bank salinity and temperature field during flood and ebb, respectively.

Observations indicate stratification limits the vertical extent of the tidal bottom boundary layer, causing a current speed maximum in the lower water column and strong veering of the velocity vector toward the bottom. The amplitude of the current speed maximum can be enhanced or reduced by mode-one internal wave motion at the $M_{2}$ frequency. Measurements indicate baroclinic tides are particularly pronounced in summer, with their phase relative to the barotropic tide varying with time.

Current measurements and bottom stress estimates are compared to results from a one-dimensional numerical model incorporating the Mellor-Yamada level 2.5 turbulence closure scheme. Model solutions underpredict the vertical extent of the turbulent boundary layer at times when stratification in the BBL is large. Typical modeled velocity profiles display a pronounced current speed maximum in the turbulent lower water column followed by abrupt adjustment to the frictionless interior, while observations indicate a more gradual transition. Numerical predictions show better agreement with the observations for times when buoyancy frequencies approach zero in a substantial portion of the $\mathrm{BBL}(\approx 15 \mathrm{~m})$ around the reversal 
from ebb to flood. However, such behavior is representative only of the second half of the May Shelf-Slope front intrusion investigated here and does not describe the onset of the intrusion or measurements representative of summer. We therefore conclude that either the MY2.5 turbulence closure scheme underestimates turbulence production in the presence of stratification as proposed earlier for weakly stratified conditions (see Chapter 2), or the vertical boundary layer structure cannot be modeled adequately with a one-dimensional model.

\subsection{Introduction}

Over wide parts of the continental shelf, bottom friction greatly determines the vertical structure of the tidal currents and large scale circulation. Friction-induced turbulent mixing creates strong current shear, which decreases with increasing distance away from the source of turbulence presented by the rough bottom.

Analysis of the tidal velocity distribution can best be performed by expressing the tidal currents in rotary coordinates, i.e., by separating the amplitude and phase information into a clockwise and a counterclockwise rotating component (e.g., Soulsby, 1983). In the absence of stratification, the vertical structure of the rotating components is defined by two scale heights (Sverdrup, 1927; Kagan, 1966; Prandle, 1982a,b; Soulsby, 1983). The smaller height $\delta^{+}$describes the counterclockwise component, whose cyclonic rotation assists the Coriolis force in balancing bottom friction and reduces the boundary layer thickness compared to the case of steady planetary flow. The larger height $\delta^{-}$describes the clockwise component, whose anticyclonic rotation opposes the Coriolis force and enhances the vertical extent of the frictionally dominated region. Both the clockwise and counterclockwise components combine to define the velocity vector and its direction of rotation, and their relative magnitudes determine which of the two scale heights dominates the current profile. Tidal current measurements from several locations have shown the rotating components display different boundary layer heights $\delta^{+}$and $\delta^{-}$, in agreement with analytical predictions (e.g., Maas and van Haren, 1987; Soulsby, 1990, Werner and Beardsley, submitted).

During stratified conditions, an additional length scale enters the problem which describes the effects of buoyancy forcing on turbulent mixing and is referred to as 
Monin-Obukhov length (e.g., Kundu, 1990). If stratification is large, the vertical extent over which the frictional and buoyancy forces balance may be significantly smaller than the BBL thickness during homogeneous conditions. Hence, the BBL cannot extend as far into the interior as in the unstratified case. Measurements have shown the result is strong current shear in and across the pycnocline (e.g., Maas and van Haren, 1987; Soulsby, 1990). This is in agreement with model results by Maas and van Haren (1987), who used a three-layer eddy viscosity distribution with reduced value in the pycnocline to approximate the observed velocity distribution. Since $\delta^{-}>\delta^{+}$, the effects of stratification on the boundary layer structure are larger for the clockwise than the counterclockwise component (e.g., Souza and Simpson, 1996; Visser et al., 1994).

In part I of this study, Werner and Beardsley (Chapter 2, hereafter WB) used moored array data and numerical models to investigate the tidal boundary layer on the southern flank of Georges Bank for nearly homogeneous conditions. Their observations show the clockwise rotating component largely determines the vertical distribution of the semidiurnal tidal currents at the 76-m deep study site. Numerical results from a two-layer eddy viscosity model and a model incorporating Mellor-Yamada level 2.5 (MY2.5) turbulence closure (Mellor and Yamada, 1974, 1982) show overall good agreement with the observed velocity profile and estimated bottom stress. However, WB found some indication that the MY2.5 model underpredicts turbulence production in the presence of stratification, even for small buoyancy frequencies $N^{2}=O\left(10^{-5}\right) \mathrm{s}^{-2}$ representative of winter: WB's conclusions are supported by Stacey et al. (submitted), who demonstrated the MY2.5 model underestimates the turbulent kinetic energy at and above the density interface in a shallow, partially-stratified tidal channel in upper San Francisco Bay. On the other hand, Simpson et al. (1996) reported good agreement between measured and modeled dissipation rates at a $90-\mathrm{m}$ deep site in the Irish Sea using a turbulence closure scheme very similar to the MY2.5, i.e., their MY2.2b. It is possible the BBL was confined to the region below the main pycnocline in Simpson et al.'s (1996) case, so that the ability of the model to incorporate the effects of large $N^{2}$ was not tested (WB). However, no information is available to either prove or disprove this hypothesis. Consequently, further investigation of the MY2.5 model is required in order to evaluate its performance during stratified conditions.

In this study, we extend the analysis from part I to investigate the impacts 
of strong stratification $\left(N^{2}=O\left(10^{-4} \mathrm{~s}^{-2}\right)\right)$ on the BBL on the southern flank of Georges Bank. Based on moored array observations, we examine the tidal current structure during times of bottom- and surface intensified $N^{2}$, the contribution of subtidal flow shear to turbulence production, and the presence of baroclinic structures resembling mode-one internal tides. From best-fit logarithmic profiles to acoustic current meter data, we compute estimates of bottom stress, bottom roughness, and frictional drag coefficients, and compare the results to the friction parameters derived for winter. The tidal boundary layer is examined numerically with a one-dimensional model incorporating the MY2.5 turbulence closure scheme. Model solutions are compared to observational data, and conclusions are drawn about the overall performance of the numerical model in the presence of stratification.

\subsection{Physical Setting and Moored Array}

The physical setting and moored array on the southern flank of Georges Bank are described in WB and shown in Fig.3.1 and Fig.3.2. Velocity, temperature, and conductivity data were taken from February 3-August 23 at Stratification Site 1 (ST1, $40^{\circ} 51.8^{\prime} \mathrm{N}, 67^{\circ} 33.5^{\prime} \mathrm{W}$ ) on the $76-\mathrm{m}$ isobath roughly $20-30 \mathrm{~km}$ upslope of the base of the SSF and $20 \mathrm{~km}$ downslope of the TMF, and February 3-August 4 at Stratification Site $2\left(\mathrm{ST} 2,40^{\circ} 57.4^{\prime} \mathrm{N}, 67^{\circ} 37.6^{\prime} \mathrm{W}\right)$ on the $69-\mathrm{m}$ isobath about $15 \mathrm{~km}$ upslope of ST1. The VMCM at $18 \mathrm{~m}$ above the bottom (mab) failed after June 21. The BASS tripod was deployed from July 11 to August 24 and started data sampling on July 12. Due to storage problems at later times, BASS velocity data are only available until August 14. The instrumental setup of the BASS tripod is similar to that of the winter deployment (WB), with identical sample rates but slightly modified heights of the five acoustic travel time current meters (pods 1-5: $0.28,0.63,1.23,2.58$, and $4.48 \mathrm{~m}$ above deck), and eight thermistors $(0.34,0.60$, $1.20,1.84,2.55,3.50,4.45$, and $6.04 \mathrm{~m}$ above deck). Sensor heights above the bottom are $2 \mathrm{~cm}$ below the above deck values to account for sinking of the tripod legs. In contrast to the winter deployment, no camera, light strobe, or battery case were attached to the BASS tripod, so that flow distortion due to these instruments could be avoided. 


\subsection{Bottom Stress}

For steady, unstratified, turbulent flow, the velocity distribution near a rough surface is described by the logarithmic law of the wall

$$
U=\frac{u_{*}}{\kappa} \ln \left(\frac{z}{z_{0}}\right),
$$

where

$$
u_{*}=\sqrt{\tau_{b}}
$$

is friction velocity, $\kappa=0.4$ is von Karman's constant, $z$ is height above bottom, $z_{0}$ is bottom roughness, and $\tau_{b}$ is magnitude of the kinematic bottom stress (dynamic stress divided by density). WB showed (1) closely describes the currents below 2.5 mab at ST1 in winter, indicating corrections that account for the effects of tidal acceleration are either small or cannot be resolved by the measurements.

The exact magnitude of vertical stratification in the logarithmic layer cannot be determined, since no salinity estimates are available below 11 mab. Temperature measurements reveal thermal stratification was $<0.010 \pm 0.001^{\circ} \mathrm{C}$ about $95 \%$ of the time between the thermistors at 0.6 and $2.5 \mathrm{mab}$ (Fig.3.3). Using typical nearbottom temperatures of $9.4^{\circ} \mathrm{C}$, and assuming salinities are vertically uniform and around 33 based on measurements at $11 \mathrm{mab}$, we obtain $N^{2}=8 \cdot 10^{-6} \mathrm{~s}^{-2}$ for $\Delta T=0.01^{\circ} \mathrm{C}$ between 0.6 and $2.5 \mathrm{mab}$. Hence, it cannot be ruled out that vertical stratification affects the near-bottom velocities to some degree, thereby causing deviations from (1) as described in Kundu (1990). In comparison, thermal stratification at 0.6-2.5 mab was indistinguishable from the thermistor accuracies $\left(0.001^{\circ} \mathrm{C}\right)$ for more than $95 \%$ of the winter analysis period discussed in WB.

Assuming (1) describes the velocity distribution to lowest order, estimates of friction velocity and bottom roughness may be obtained from the instantaneous best-fit logarithmic profiles to BASS current measurements. Similar to the winter analysis performed in WB, we used pods $1-4(0.26-2.56 \mathrm{mab})$ to compute the fits. Unlike the winter case, correction of the velocity measurements for offset residuals did not improve the summer data and was not applied. Logarithmic fits with standard errors $>2.0 \mathrm{~cm} \mathrm{~s}^{-1}$ or deviations of estimated to measured current speeds. $>2.0 \mathrm{~cm} \mathrm{~s}^{-1}$ were excluded from the analysis, which limits the data set 
to $98 \%$ of its original length from July 12 -August 14 . The linear regression coefficients of all retained burst-averaged velocities at pods 1-4 to the logarithm of height above bottom are $\geq 0.95(\geq 0.90)$ more than $87 \%(95 \%)$ of the time. These percentages are comparable to those for the winter period ( $\geq 0.95$ and $\geq 0.90$ more than $79 \%$ and $97 \%$ of the time, respectively; see also WB), indicating that despite the presence of vertical stratification, (1) represents a good approximation for the velocity distribution near the bottom.

Estimated friction velocities are shown as a function of time in Fig.3.4a, with missing values filled in by linear interpolation. The $95 \%$ confidence limits of the fits $\Delta u_{*}^{95}$ are $O(0.1-1) \cdot u_{*}$ (Fig.3.4b). Taking the record average gives $u_{*}=$ $1.1 \pm 0.4 \mathrm{~cm} \mathrm{~s}^{-1}$, where the standard deviation $0.4 \mathrm{~cm} \mathrm{~s}^{-1}$ mostly represents tidal fluctuations. For the winter period February 11-March 11, we found almost identical values $u_{*}=1.2 \pm 0.4 \mathrm{~cm} \mathrm{~s}^{-1}$.

Friction velocities increase almost linearly with current speed $U$ (Fig.3.5), in agreement with the quadratic drag law

$$
\tau_{b}=c_{D} U^{2}
$$

where

$$
c_{D}=\left[\frac{\kappa}{\ln \left(\frac{z}{z_{0}}\right)}\right]^{2},
$$

derived from the logarithmic law of the wall. Least-squares fits of $u_{*}^{2}$ versus $U^{2}$ using BASS and VMCM measurements at 0.24-6 mab give drag coefficients that are within $1-20 \%$ of those obtained from the winter data, with largest differences between the two investigation periods occuring very close to the bottom (Table 3.1). This result suggests the drag coefficients are approximately constant throughout the year, so that bottom stress may be estimated from the quadratic drag law during times when BASS data are not available. Comparison of bottom stress estimates from logarithmic fits and the quadratic drag law at 6 mab support this conclusion (Fig.3.6). Fig.3.6.a shows estimates of the tidal $\left(M_{2}\right)$ bottom stress vector are very similar using either computation method. Results displayed in the Figure were obtained from harmonic analysis of the stress vector, where estimates $u_{*}^{2}$ from logarithmic fits were assigned the direction of the vertically averaged flow at pods 1-3 (0.26-1.21 mab). The tidal analysis was performed using Godin's harmonic method 
(Foreman, 1978) on time-windows of eight days length, with the temporal spacing of the window-centerpoints set to two days. The observed amplitude modulations on temporal scales of about 15 and 30 days represent the small and large springneap cycles with periods of 14.8 and 27.6 days, respectively, and are caused by the superposition of the semidiurnal $S_{2}$ and $N_{2}$ constituents on the $M_{2}$ tidal forcing. For the subtidal component (temporal variations $>33 \mathrm{hrs}$ ), similar comparison shows bottom stress estimates from both methods are in good agreement for the summer BASS deployment (Fig.3.6b). During the winter deployment, large deviations occur in the cross-bank component, while the along-bank estimates are close. The discrepancy between the two estimation methods in the cross-bank direction in winter is most likely due to flow distortion caused by the battery powering the camera and light attached to the winter BASS tripod (Werner, 1996).

Bottom roughness estimates obtained from the logarithmic fits lie between $z_{0}=$ $10^{-14}-251 \mathrm{~mm}$ (Fig.3.7a), with $95 \%$ confidence intervals $\Delta z_{0}^{95}$ that are $O\left(z_{0}\right)$ (Fig.3.7b). Due to the wide range of $z_{0}$ values, it is difficult to infer an accurate estimate of bottom roughness based on the logarithmic fits alone. Meaningful values may be derived from (4) and are $z_{0}=0.56,0.67,0.62$, and $0.69 \mathrm{~mm}$ based on the drag coefficients at BASS pods 1-4 (Table 3.1), respectively. A representative bottom roughness according to these values is $z_{0}=0.6 \mathrm{~mm}$, in good agreement with $z_{0}=0.5 \mathrm{~mm}$ inferred from winter data (WB). This result also follows from the lack of significant variation in drag coefficient between winter and summer noted above.

\subsection{Shelf-Slope Front (SSF) Intrusion (May 6-23)}

On May 6, moored array measurements at ST1 show an intrusion of high temperature and salinity water which persisted until approximately May 28. SeaCAT temperature and salinity data (Fig.3.8) together with TPOD and VMCM temperature measurements (not shown) indicate the intrusion was bottom-trapped below $35 \mathrm{mab}$ before May 14 and extended to the surface at later times. Salinities at 11 mab increased from about 32.6 preceding the intrusion to values as large as 35.2 on May 6-7. Such behavior suggests the base of the SSF passed the 76-m isobath, and warm saline Slope Water with characteristic salinities $35<S<36$ displaced the fresher Georges Bank Water defined by $32.2<S<33$ (Flagg, 1987). On 
May 8, the SSF receded and salinities dropped to about 32.6 , immediately followed by a second, longer intrusion (May 9-28) with near-bottom salinities that reached 34.7-34.8 (May 11-22). Largest salinities at heights $\geq 29.5$ mab were $34.2-34.8$ with magnitudes decreasing upward, representative of conditions in the transition region between the Georges Bank and Slope Waters.

Moored data from ST2 show the base of the SSF reached ST2 around May 15, causing salinities of $34.3-34.5$ at 7 and 39 mab between May 15-17 (Fig.3.9). Salinity measurements suggest the SSF intrusion only marginally affected the nearsurface waters $(z \geq 59 \mathrm{mab})$ at ST2.

\subsubsection{Bottom-Trapped Phase (May 6-14)}

During the bottom-trapped phase of the SSF intrusion (May 6-14, Fig.3.8), temperature-salinity $(T-S)$ characteristics display almost linear behavior at 11 and $29.5 \mathrm{mab}$ (Fig.3.10). This behavior suggests salinity can be inferred from temperature measurements in the lower water column. Least-squares fits of hourly averaged SeaCAT data to the slope $S=a \cdot T+S_{0}$ were performed for time-windows of 24-hrs length, and the results were used to estimate salinity at the TPOD heights 8.5 and $14.5 \mathrm{mab}$. To obtain a continuous time-series of $S$, the centerpoint of the time-window was passed through the investigation period such that the coefficients $a$ and $S_{0}$ were determined on an hourly basis. The average standard error of all fits is 0.02 for both the salinities at 11 and 29.5 mab. Linear interpolation of $a$ and $S_{0}$ with depth allows estimation of $S$ between adjacent SeaCAT sensors, i.e., at 14.5 mab. At depths larger than that of the lowest SeaCAT (11 mab), the coefficients at 11 mab were used to compute salinity.

Time-mean profiles for May 6-14 display a large increase of temperature, salinity, and potential density toward the bottom. The observed gradients mark the transition from the cold, fresh Georges Bank water to the underlying warm, saline water of the SSF (Fig.3.11a-c). Buoyancy frequencies average $N^{2} \approx 2.5 \cdot 10^{-4} \mathrm{~s}^{-2}$ near $10 \mathrm{mab}$ (Fig.3.11d), a value larger than typical late-summer stratification at the same height $\left(N^{2} \approx 10^{-4} s^{-2}\right)$.

Fig.3.12 shows variations of temperature, salinity and potential density as a function of tidal flow direction (Fig.3.13), where the term tidal refers to temporal variations $<33 \mathrm{hrs}$ (diurnal and semidiurnal tides). Measurements are shown for the SeaCATs at 11-70 mab and the TPOD at $8.5 \mathrm{mab}$, with salinities at the TPOD 
height estimated as described above. Results indicate tidal variations were largest in the lower water column, with $\Delta T_{M_{2}} \approx 4^{\circ} \mathrm{C}, \Delta S_{M_{2}} \approx 1.5$, and $\Delta \sigma_{\theta M_{2}} \approx 0.4$ at 8.5-11 mab. Peak values of $T, S$, and $\sigma_{\theta}$ are observed at the end of flood $\left(\alpha=90^{\circ}\right)$, indicating advection of mixtures of warmer, saltier and denser Slope Water occured when the tidal flow was on-bank. In the upper water column at $50 \mathrm{mab}$ and above, variations of $T, S$, and $\sigma_{\theta}$ are hardly noticeable and at least one order of magnitude smaller than at depth. The observed behavior of temperature, salinity, and density is consistent with strong off-bank gradients caused by the on-bank intrusion of the SSF. Taking the tidal excursion $\ell_{M_{2}}=\frac{2 U}{\sigma} \approx 6 \mathrm{~km}$, where $U=0.4 \mathrm{~m} \mathrm{~s}^{-1}$ is the cross-bank amplitude of the $M_{2}$ current (WB), and $\sigma=1.4 \cdot 10^{-4} \mathrm{~s}^{-1}$ is the $M_{2}$ frequency, characteristic off-bank density gradients in the lower water column are $\frac{\Delta \sigma_{\theta M_{2}}}{\ell_{M_{2}}} \approx 0.07 \mathrm{~km}^{-1}$. This corresponds to a thermal wind shear of $\approx 7 \mathrm{~cm} \mathrm{~s}^{-1}$ over $10 \mathrm{~m}$ vertical distance, in qualitative agreement with the observed subtidal along-bank currents at $z<30 \mathrm{mab}$ (Fig.3.14a). Using data from the ST1 and ST2 moorings gives $\frac{\Delta \sigma_{\theta}}{\Delta x} \approx 0.03 \mathrm{~km}^{-1}$ near 14 mab, where $\Delta x=12 \mathrm{~km}$ is the separation distance between ST1 and ST2, and $\Delta \sigma_{\theta}$ is the cross-bank density difference. Thus, $\frac{\Delta \sigma_{\theta_{M_{2}}}}{\ell_{M_{2}}} \approx 2 \frac{\Delta \sigma_{\theta}}{\Delta x}$, indicating the horizontal spacing between ST1 and ST2 is too large to fully resolve the local gradients during the bottom-trapped phase of the SSF intrusion.

Buoyancy frequency estimates between 8.5-11 mab are largest around the reversal from ebb to flood $\left(N^{2} \approx 6 \cdot 10^{-4} \mathrm{~s}^{-2}, \alpha=270^{\circ}\right)$ when vertical gradients $\frac{\Delta S}{\Delta z}$ and $\frac{\Delta T}{\Delta z}$ are most negative, and smallest around the reversal from flood to $\mathrm{ebb}$ $\left(N^{2} \approx 0.7 \cdot 10^{-4} \mathrm{~s}^{-2}, \alpha=90^{\circ}\right)$ when $\frac{\Delta S}{\Delta z}$ and $\frac{\Delta T}{\Delta z}$ are close to zero (Fig.3.15a-c). This behavior suggests the near-bottom waters on-bank of ST1 are strongly stratified, while stratification is weaker over the outer bank. The situation is reversed at heights $>14.5 \mathrm{mab}$, where stratification is greatest during the second half of flood and first half of ebb $\left(\alpha=0-180^{\circ}\right)$, indicating the off-bank waters are more stratified than the region on-bank of ST1. As shown schematically in Fig.3.16a, the observed variation suggests the base of the SSF intersects ST1 between $11-14.5$ mab. The result is strong near-bottom stratification on-bank of ST1 which is advected off-bank during ebb. At heights $>14.5 \mathrm{mab}$, the interface between the warm, saline slope water and cold, fresh bank water is located off-bank of ST1 and advected on-bank during flood.

The effects of strong stratification on turbulent mixing can be investigated by 
performing a Richardson number analysis similar to the one described in WB. Here we follow WB's approach to display the percentage of events $R i<R i_{c}$ for a given time period, where $R i_{c}$ is a critical Richardson number. The choice of $R i_{c}$ is ambiguous and depends to some degree on the vertical scales resolved by the measurements. WB found that for buoyancy frequencies representative of winter $\left(N^{2}=O\left(10^{-5}\right) \mathrm{s}^{-2}\right)$, the overturning scale of the largest turbulent eddies was $L_{B} \approx$ $11 \mathrm{~m}$ and several meters smaller than the distance between adjacent SeaCATs at mid-depth. For this reason, WB chose $R i_{c}=0.5$ rather than the commonly used stability criterion $R i_{c}=0.25$, whose application requires the overturning scales can be resolved. In the present case, we find $N^{2}=O\left(10^{-4}\right) \mathrm{s}^{-2}$ (Fig.3.11d). Since $L_{B} \propto\left(\epsilon / N^{3}\right)^{1 / 2}$ (Gregg, 1993, Ozmidov, 1965; WB), where $\epsilon$ is the turbulent dissipation rate, the increase of $N^{2}$ from winter to. spring results in a reduction of $L_{B}$ by almost one order of magnitude. In addition, $\epsilon$ is expected to decrease as stratification increases, yielding even smaller $L_{B}$. The inference is taking $R_{c}=$ 0.25 as a criterion to describe the transition from molecular to turbulent mixing is inappropriate. To be consistent with the analysis presented by WB, we chose $R_{c}=0.5$ as a representative critical value. The Richardson number analysis follows WB in all aspects, with the minor differences that a) hourly averaged SeaCAT data were used, b) density estimates at several TPOD heights are included, and c) the Monte Carlo simulation applied for error estimation assumes the characteristic uncertainty $\sigma_{\sigma_{\theta}}=0.03$ based on $T-S$ diagrams representative of spring and summer. To examine the contribution of subtidal flow shear on turbulent mixing, Richardson numbers were computed from the velocity shear of the total currents as well as high-passed currents with time scales $<33 \mathrm{hrs}$ (diurnal and semidiurnal tides).

Fig.3.15d shows the percentage of events $R i<0.5$ varies with flow direction. Between 8.5-11 mab, $R i<0.5$ about 10-70\% of the time, with the largest number of occurrences at the end of flood $\left(\alpha=90^{\circ}\right)$, and the least occurrences at the end of ebb $\left(\alpha=270^{\circ}\right)$. The observed $R i$-variation mirrors the tidal cycle of $N^{2}$ (Fig.3.15c). Results also indicate there are obvious differences between the percentages $R i<$ 0.5 derived from computations using the total and the tidal current shear. These differences are generally negative when the tidal alongbank component is negative $\left(\alpha<180^{\circ}\right)$, and positive when the tidal alongbank component is positive $\left(\alpha>180^{\circ}\right)$. The explanation lies in the predominant direction of the subtidal flow: for more 
than $95 \%$ of the investigation period May 6-14, the subtidal flow is oriented toward $\alpha=250 \pm 40^{\circ}$. Hence, the subtidal and tidal along-bank shear add (subtract) for $\alpha>180^{\circ}\left(\alpha<180^{\circ}\right)$, resulting in more (less) turbulence production. The effects of subtidal flow shear are most pronounced for $\alpha<180^{\circ}$, while large stratification during the second half of the tidal cycle $\left(\alpha>180^{\circ}\right)$ causes $R i>0.5$ most of the time with or without consideration of the subtidal currents. Nevertheless, shear estimates indicate the subtidal flow is an important contributor to turbulence production at the reversal from ebb to flood: from velocity measurements we compute $95 \%$ of the subtidal along-bank flow shear between 6-12 mab is within $9 \pm 4 \cdot 10^{-3} \mathrm{~s}^{-1}$. In comparison, the tidal flow shear at the same height is $12 \pm 8 \cdot 10^{-3} \mathrm{~s}^{-1}$ for flow directions that are roughly aligned with the $+y$-direction $(\alpha=260-280)^{\circ}$. Thus, both the subtidal and tidal current shear are of comparable magnitude and equally important for turbulence production. Around the reversal from flood to ebb, the tidal current shear between $6-12 \mathrm{mab}$ is about $14 \pm 6 \cdot 10^{-3} \mathrm{~s}^{-1}$ and opposes the along-bank shear of the subtidal currents. At the same time, buoyancy frequencies at $8.5-11 \mathrm{mab}$ are weak, so that despite the reduction of the combined tidal and subtidal shear events $R i<0.5$ are frequent.

Between 14.5-29.5 mab in the region above the intersection of the SSF with ST1, the percentage of $R i<0.5$ approaches $0 \%$ toward the end of flood ( $\alpha=90^{\circ}$ ) when $N^{2}$ is large, and about $40 \%$ around the end of ebb $\left(\alpha=270^{\circ}\right)$ when $N^{2}$ is small (Fig. 3.15c,d). The less frequent occurrence of $R i<0.5$ compared to the region below reflects the decrease in current shear with increasing distance from the bottom. Fig.3.15d shows the subtidal flow may enhance turbulence production when the tidal and subtidal flow shear add and vertical stratification is small $\left(\alpha>180^{\circ}\right)$. For reasons similar to those explaining the observations at 8.5-11 mab, results from $R i$-number computations with and without consideration of the subtidal current shear are almost identical at times when $N^{2}$ is large $\left(\alpha<180^{\circ}\right)$.

At 29.5-50 mab, events $R i<0.5$ display directional dependence that clearly mirrors the observed variation of $N^{2}$. Percentages of $R i<0.5$ reach about $90 \%$ at the end of $\mathrm{ebb}\left(\alpha=270^{\circ}\right)$ when the tidal currents are at maximum and vertical stratification is very small (Fig.3.15c,d). Results based on the total and tidal flow shear are not significantly different for all flow directions, consistent with time-mean flow profiles indicating subtidal flow shear is weak at mid-depth (Fig.3.14a).

For $z=50-65 \mathrm{mab}, R i<R i_{c}$ about $10-50 \%$ of the time. Most events 
$R i<R i_{c}$ are caused by subtidal flow shear and may be due to the effects of wind mixing. At greater heights (65-70 mab), percentages $R i<0.5$ exceed $70 \%$ independent of flow direction. This behavior suggests turbulence production is large in the near-surface waters where stratification is weak, most likely as a consequence of strong surface wind stress with mean magnitude $\bar{\tau}_{W} \approx 1.6 \mathrm{~cm}^{2} \mathrm{~s}^{-2}$. It is unclear why differences between results based on tidal and total shear estimates at 65$70 \mathrm{mab}$ are not larger than observed. Wind-induced flow shear has greatest effects on subtidal time scales, so that a behavior qualitatively similar to the observations at 50-65 mab might be closer to expectation. However, with $95 \%$ confidence limits $\Delta_{N^{2}}^{95}$ that are typically larger than the $N^{2}$ estimates themselves (Fig.3.15c), it is likely the measurements cannot distinguish between $R i<0.5$ events caused by the tidal and the total current shear.

\subsubsection{Surface-Intensified Phase (May 14-22)}

During the second phase of the SSF intrusion (May 14-22), $T-S$ characteristics display nearly linear behavior at heights $\leq 50 \mathrm{mab}$ (Fig.3.17). Based on this behavior, salinities at $8,14.5,35$ and 44.5 mab were inferred from TPOD temperature measurements as described for the bottom-trapped phase. The average standard error of all fits is $0.02,0.03$, and 0.05 for salinities at the SeaCAT heights 11, 29.5, and 50 mab, respectively. Time-averaged profiles show a continuous decrease of temperature and salinity toward the surface (Fig.3.11a,b), with a weak temperature reversal between $67-76 \mathrm{mab}$ indicative of solar heating. Vertical stratification is surface-intensified, with $N^{2} \approx 1.5 \cdot 10^{-4} \mathrm{~s}^{-2}$ in the upper water column (Fig.3.11d).

Characteristic variations of temperature, salinity and potential density during a tidal cycle are comparable at all heights $\left(\Delta T_{M_{2}} \approx 2^{\circ} \mathrm{C}, \Delta S_{M_{2}} \approx 0.5\right.$ and $\Delta \sigma_{\theta M_{2}} \approx 0.2$ ), with somewhat smaller values near the bottom (Fig.3.18). Divided by the tidal excursion $\ell_{M_{2}}=6 \mathrm{~km}$, these values yield the off-bank density gradient $\frac{\Delta \sigma_{\theta M_{2}}}{l_{M_{2}}} \approx 0.03 \mathrm{~km}^{-1}$, in reasonable agreement with estimates obtained from ST1 and ST2 data suggesting $\frac{\Delta \sigma_{\theta}}{\Delta x} \approx 0.03 \mathrm{~km}^{-1}$.

Fig.3.19b shows the largest (negative) vertical salinity gradients occur toward the end of ebb/ beginning of flood $\left(\alpha=270^{\circ}\right)$ in the lower water column $(z<$ $29.5 \mathrm{mab})$, and toward the end of flood/ beginning of ebb $\left(\alpha=90^{\circ}\right)$ near the surface $(z>50 \mathrm{mab})$. Most (least) negative $\frac{\Delta S}{\Delta z}$ coincide with most (least) negative 
$\frac{\Delta T}{\Delta z}$ (Fig.3.19a,b). The observed behavior indicates the SSF has moved on-bank and intersects ST1 at mid-depths between 29.5 and 50 mab (Fig.3.16b). Similar to May 6-14, stratification is greatest when $\frac{\Delta S}{\Delta z}$ is most negative (Fig.3.19b,c).

Results from Richardson number analysis show $R i<0.5$ about $40-100 \%$ of the time at $z<29.5 \mathrm{mab}$ (Fig.3.19d), indicating turbulence intensity is large during all phases of the tidal cycle. The effects of subtidal flow shear are most pronounced in the lower water column $(z<29.5 \mathrm{mab})$, where events $R i<0.5$ increase by up to $50 \%$ for $\alpha>180^{\circ}$, if the subtidal shear is included in the analysis. Similar to May 6-14, the around-bank circulation is roughly along-isobath and to the southwest, so that the subtidal currents add to the along-bank component of the tidal flow for $\alpha>180^{\circ}$. Analysis of current data shows the subtidal current shear is within $6 \pm 2 \cdot 10^{-3} \mathrm{~s}^{-1}$ at 6-12 mab during more than $95 \%$ of the investigation period, compared to $6 \pm 2 \cdot 10^{-3} \mathrm{~s}^{-1}$ for tidal currents that are directed toward $\alpha=260-280^{\circ}$. These results indicate subtidal flow shear is an important contributor to turbulence production near the lower boundary around the reversal from ebb to flood, coinciding with those times in the tidal cycle when stratification in the BBL is largest (Fig.3.19c). The opposing effects of the subtidal and tidal current shear near $\alpha=90^{\circ}$ do not influence the percentage of $R i<0.5$ to a significant degree, most likely because $N^{2}$ approaches zero in the lower water column at the end of flood (Fig.3.19c,d). At heights 35-65 mab, turbulence production is weak since stratification is large throughout the tidal cycle, and the current shear is smaller than at depths closer to the lower boundary. Hence, the percentage of $R i<0.5$ lies between $0-30 \%$, a range significantly less than that in the highly turbulent region below. $R i<0.5$ occurs more frequently $(10-50 \%)$ between $65-70$ mab, most likely in conjunction with intermittent events of large surface wind stress that have peak values $1-3 \mathrm{~cm}^{2} \mathrm{~s}^{-2}\left(\bar{\tau}_{W} \approx 0.5 \mathrm{~cm}^{2} \mathrm{~s}^{-2}\right)$.

\subsubsection{The $\mathrm{M}_{2}$ Current Ellipse}

The tidal current analysis was carried out using Godin's harmonic method (Foreman, 1978) on time windows of 8-days length with the Rayleigh criterion one. To allow for easier comparison of the velocity distribution at different times during the spring-neap cycle, we predicted the tidal currents based on the analysis of winter data (February 11-March 11), and found the $M_{2}$ amplitudes $A_{\text {pred }}$ of the predicted time-series for the same 8-day time-windows. The estimated $M_{2}$ amplitudes of 
the measured time-series were normalized by the factor $\frac{A_{W}}{A_{\text {pred }}}$, where $A_{W}$ is the $M_{2}$ current amplitude from tidal analysis of the winter period.

Results are presented in rotary components as described by WB, where the amplitudes $R^{+}$and $R^{-}$were normalized following the procedure described above. Phase angles and ellipse inclination are given as relative phase-advance of the velocity vector and rotation of the major axis, respectively, where the topmost seven VMCMs were used to define the reference value. Values $\Delta \phi^{+}>0$ and $\Delta \phi^{-}<0$ describe a phase-lead of the anticlockwise and clockwise component as the bottom is approached, respectively, and $\Delta \phi<0$ represents a phase advance of the $M_{2}$ velocity vector with respect to the surface. Negative $\Delta \theta$ defines clockwise rotation of the major axis toward the bottom. BASS current data were not available during the May SSF intrusion event.

Tidal analysis of VMCM measurements taken during the bottom-trapped (May 614) and surface intensified (May 14-22) phases of the SSF intrusion suggests stratification causes a maximum $R_{M a x}^{-}$in the lower water column, followed by a region of small velocity shear $(z>40-50 \mathrm{mab})$, in agreement with earlier observations by Maas and van Haren (1987) in the North Sea (Fig.3.20a). For May 6-14, amplitudes $R^{+}$show a monotonic increase between 18 and $45 \mathrm{mab}$ in the region below and across the pycnocline, which separates the stratified near-bottom waters from the nearly homogeneous upper water column (Fig.3.11d). Such behavior together with near-surface amplitudes $R^{+}\left(R^{-}\right)$that are larger (smaller) than their corresponding winter values may be indicative of internal wave motion at the $M_{2}$ frequency. For May 14-22, amplitudes $R^{+}$are almost identical to the winter values, indicating stratification affects the anticlockwise component to a lesser degree than the clockwise component. This is consistent with scaling arguments suggesting the anticlockwise boundary layer does not extend as far into the stratified interior as the clockwise boundary layer (Souza and Simpson, 1996). Results for the southern flank of Georges Bank representative of winter indicate $\frac{\delta^{-}}{\delta^{+}} \approx \frac{\sigma+f}{\sigma-f} \approx 5$ may be a characteristic ratio for the clockwise and anticlockwise boundary layer thicknesses, if the water column is nearly homogeneous (WB).

Phase angles $\Delta \phi^{-}$shown in Fig.3.20c rotate by about $-30^{\circ}$ between $30 \mathrm{mab}$ and the sea floor during the SSF intrusion, as opposed to about $-15^{\circ}$ for the near-homogeneous period February 11-March 11. This behavior indicates bottom friction may be balanced over a smaller height due to the stratified interior. Veering 
angles $\Delta \phi^{+}$are weak $\left(<10^{\circ}\right)$, consistent with earlier conclusions that the counterclockwise boundary layer height is small compared to the clockwise boundary layer (Fig.3.20b). Fig.3.20c shows the clockwise rotation of the major axis during the SSF intrusion exceeds the rotation observed in winter by $\approx 10^{\circ}$ at heights $<30$ mab (Fig.3.20e). Similar conclusions apply to the phase of the velocity vector near the bottom which leads the velocities at $30-40$ mab by $15-20^{\circ}$, as opposed to about $10^{\circ}$ during winter (Fig.3.20f).

\subsubsection{Numerical Model}

A one-dimensional numerical model with vertical grid spacing $0.5 \mathrm{~m}$ (WB) was run to investigate the effects of strong stratification on vertical mixing during the SSF intrusion. The model uses the Mellor-Yamada level 2.5 (MY2.5) turbulence closure to compute the vertical transfer of momentum (Mellor and Yamada, 1974, 1982). Based on our data analysis, the specified bottom roughness was $z_{0}=0.6 \mathrm{~mm}$. Following WB, the tidal pressure forcing was obtained from vertical integration of the momentum equations and is representative of the $M_{2}$ pressure gradient during the period investigated. To explore the impacts of a given density distribution on the tidal velocity structure, the density profile was specified as a function of time and the evaluation of the density equation was omitted. The effects of subtidal flow shear on turbulent mixing were investigated by adding a time-independent flow to the numerical velocities after solving the tidally-forced momentum equations. This approach was taken since the baroclinic pressure gradient acting upon the subtidal circulation is not known, making the implementation of appropriate model forcing difficult. The sum of the tidally forced and mean currents entered the turbulent kinetic energy and length scale equations, which directly lead to the computation of the vertical mixing coefficients. After evaluation of the mixing coefficients, the model stepped forward in time and the specified mean flow was subtracted from the total velocities. Thus, only the tidally-forced velocities evolved in time while the mean flow remained fixed. Specifying the mean flow field as described above does not account for the surface boundary condition of an applied wind stress. Subtidal flow profiles indicate wind forcing caused large flow shear in the upper 10-15 $\mathrm{m}$ of the water column (Fig.3.14), a conclusion which is supported by numerical experiments (results not shown). This suggests turbulence due to wind stress is spatially separated from the region of significant tidal current shear, which based on our tidal 
analysis extends to about 40-50 mab at the 76-m deep ST1 site (Fig.3.20).

\subsubsection{Model-Data Comparison}

Numerical results representative of May 6-14 are shown together with data in Fig.3.22. Vertical stratification was specified according to Fig.3.21a, with largest $N^{2}$ below (above) $z=12$ mab occurring at the reversal from ebb to flood (flood to ebb) in agreement with our observations (Fig.3.15c). The numerical model was run for barotropic pressure forcing representative of May 6-14 with and without a superimposed $M_{2}$ baroclinic pressure forcing corresponding to a mode-one internal wave (Appendix). Direction and magnitude of the baroclinic pressure gradient were chosen such that the free-stream amplitudes $U_{t} a u E C O M 1 d_{1} 34 p 4_{1} 42 p 4 v a r M a j$ remain unchanged compared to the barotropic case, while the surface values $U_{M i n}$ are reduced by about $5 \mathrm{~cm} \mathrm{~s}^{-1}$ (Fig.3.22d). Model-data comparison of current amplitudes in the upper water column supports the conclusion that mode-one internal waves may modify the observed velocity distribution (Fig.3.22a,d).

For both purely barotropic and barotropic together with baroclinic forcing, model solutions predict a turbulent boundary layer at heights $<16$ mab with eddy viscosities $K$ that reach about $0.01 \mathrm{~m}^{2} \mathrm{~s}^{-1}$ (Fig.3.24a). At greater heights, $K=\nu$ and turbulent mixing is shut down. Model results predict a pronounced current speed maximum near 12 mab whose magnitude is enhanced by the presence of internal tides (Fig.3.22a,d). Its height above bottom is lower than indicated by the observations, which suggest $z=18$ mab or higher as the approximate location of the observed maximum $R_{M a x}^{-}$(Fig.3.20a). The predicted current speed maximum is followed by a region of strong shear below the top of the boundary layer (12 mab $<z<15 \mathrm{mab}$ ), marking the transition to the frictionless interior. Such behavior is not supported by our observations, which suggest a more gradual transition from the turbulent boundary layer to the upper water column. Numerical results for $\Delta \theta$ and $\Delta \phi$ indicate clockwise rotation of the major axis toward the sea floor and pronounced phase-lead of the near-bottom velocities in agreement with measurements (Fig.3.22e,f). Bottom stress estimates from the model are $\tau_{b_{M a j}}=1.3 \mathrm{~cm}^{2} \mathrm{~s}^{-2}$ and $\tau_{b_{M i n}}=-0.4 \mathrm{~cm}^{2} \mathrm{~s}^{-2}\left(\tau_{b_{M a j}}=1.2 \mathrm{~cm}^{2} \mathrm{~s}^{-2}\right.$, $\tau_{b_{\text {Min }}}=-0.9 \mathrm{~cm}^{2} \mathrm{~s}^{-2}$ ) for purely barotropic (barotropic together with mode-one baroclinic) forcing, as opposed to the larger values $\tau_{b_{M a j}}=1.7 \pm 0.2 \mathrm{~cm}^{2} \mathrm{~s}^{-2}$ and $\tau_{b_{\text {Min }}}=-0.8 \pm 0.1 \mathrm{~cm}^{2} \mathrm{~s}^{-2}$ obtained from the observations and normalized in 
the same manner as described for the current amplitudes. Numerical experiments show the bottom stress estimates are somewhat sensitive to the height of the re-

gion $N^{2}=0$ near the bottom. For example, extending the mixed layer height from 3 to 6 mab increases the bottom stress estimates by about 15\%, but does not significantly change the $M_{2}$ current distribution. Model predictions for the tidal velocity profiles are not affected by the implementation of mean flow shear according to Fig.3.14a (results not shown).

Numerical solutions for May 14-22 are presented in Fig.3.23 for vertical stratification according to the time-mean and the time-mean superimposed by tidal variations shown in Fig.3.21b. Tidal variations of $N^{2}$ were chosen such that largest buoyancy frequencies above (below) 45 mab occurred at the reversal from flood to ebb (ebb to flood). The applied $M_{2}$ pressure forcing was barotropic. Both a time-constant and time-varying $N^{2}$ distribution yield similar results, with some indication that the tidal boundary layer thickness increases by about $5 \mathrm{~m}$ and the transition from turbulent boundary layer to the free-stream is more gradual if stratification varies on tidal time scales (Fig.3.23a,d). This is because the turbulent eddy viscosities in the lower water column are large when stratification is near its minimum, resulting in an overall thicker boundary layer than for a $N^{2}$ distribution representative of the time-mean (Fig.3.24b,c). Similar to May 6-14, the model under-predicts the height of the observed current speed maximum by about $7 \mathrm{~m}$ (Fig.3.23a,d). Bottom stress amplitudes from the model are almost identical for time-constant and time-varying $N^{2}$, with predicted magnitudes $\tau_{b_{M a j}}=1.8 \mathrm{~cm}^{2} \mathrm{~s}^{-2}$ and $\tau_{b_{M i n}}=-0.9 \mathrm{~cm}^{2} \mathrm{~s}^{-2}$ that are within $1 \%$ and $6 \%$ of the observed values, respectively. Similar to numerical experiments for May 6-14, model results do not change significantly if a mean flow according to Fig. $3.14 \mathrm{~b}$ is added to the tidal forcing (results not shown).

\subsection{Internal $\mathrm{M}_{2}$ Tide}

In the previous section, we suggested internal tides may be present at the $M_{2}$ frequency during times of strong stratification. The baroclinic structure of these waves modifies the velocity distribution of the $M_{2}$ current ellipse and is largely described by the first vertical mode. Tidal analysis of BASS and VMCM current measurements provides further evidence of internal tides in summer, when stratification was 
large everywhere in the water column. The relative phase of the barotropic and baroclinic tides depends on factors such as wave propagation speed and the location of internal wave generation. The location of the generation region on the southern flank is not known, but is likely to be affected by the position of the SSF. Since the SSF is not a stationary feature and stratification varies on both tidal and subtidal time-scales, the relative phase of the barotropic and baroclinic $M_{2}$ pressure forcing changes with time. Analysis of unaveraged ST1 SeaCAT data (sample rate $1.5 \mathrm{~min}$ ) also suggests the presence of internal wave solitons in summer with wave periods of $5-10 \mathrm{~min}$. The solitary wave trains pass ST1 within $0.5-2 \mathrm{hrs}$ and occur regularly after the reversal from ebb to flood, when they are advected on-bank by the tidal flow. Since the focus of this paper is on tidal motion at the $M_{2}$ frequency, the high frequency internal solitary waves observed over the southern flank in summer will be discussed elsewhere.

Two examples representative of mode-one internal tides are discussed here: July 1826 and August 6-14. VMCM data at 18 mab were not available during these periods. In both cases, time-averaged stratification profiles show an almost monotonic increase toward the surface (Fig.3.25d). Stratification was computed based on SeaCAT data, since $T$ and $S$ were not sufficiently correlated to allow estimation of salinity from temperature measurements. The period August 6-14 describes a surface-trapped SSF intrusion event with salinities at $z \geq 65$ mab that exceed those near the bottom (Fig.3.25c).

\subsubsection{The $M_{2}$ Current Ellipse}

Results of the tidal current analysis for July 18-26 and August 6-14 are shown in Fig.3.26-27. Between July 18-26, velocities increase at heights $>50 \mathrm{mab}$, indicative of a mode-one internal wave whose near-surface velocities are roughly in phase with the surface velocities of the barotropic tide (Fig.3.26a,d). For August 614 , the tidal velocities decrease upward for $z>30$ mab, suggesting the barotropic and baroclinic pressure forcing in the upper water column lag each other by about $180^{\circ}$ (Fig.3.27a,d). Based on our stratification estimates in Fig.3.25d, we computed the vertical structure of the mode-one horizontal velocity amplitudes assuming frictionless flow (Appendix) and found the height of baroclinic flow reversal is near 52 and 44 mab for July 18-26 and August 6-14, respectively (Fig.3.28).

Tidal velocity profiles for July 18-26 show no evidence of a current speed maxi- 
mum in the lower water column (Fig.3.26a,d). It is possible such a maximum occurs between 12 and 30.5 mab, where current data are not available. The maximum may further be reduced by the opposing effect of the barotropic and baroclinic pressure gradient below mid-depths, which weakens the current amplitude compared to the case of barotropic forcing. Amplitudes $R^{+}$and $R^{-}$show a maximum at 30.5 mab for August 6-14 (Fig.3.27a), when the mode-one internal tide acts to enhance the velocity amplitudes in the lower water column (Fig.3.28).

Both periods exhibit strong phase veering $\Delta \phi^{-} \approx-30^{\circ}$ at $z<30 \mathrm{mab}$ (Fig.3.26c, 27c). This is consistent with observations during the May SSF intrusion and suggests stratification may limit the vertical extent of the turbulent boundary layer. The results are pronounced phase-lead of the velocity vector and rotation of the major axis toward the bottom as suggested by Fig.3.26e,f and Fig.3.27e,f $\left(\Delta \phi \approx-20^{\circ}, \Delta \theta \approx-15^{\circ}\right)$.

\subsubsection{Model-Data Comparison}

The numerical model was run for a semidiurnal barotropic pressure gradient with and without the superimposed mode-one pressure forcing corresponding to the cross-bank velocity structure shown in Fig.3.28. Surface amplitudes of the internal tide were estimated from the data and correspond to velocity amplitudes of $6(5) \mathrm{cm} \mathrm{s}^{-1}$ for July 18-26 (August 6-14). Vertical stratification was specified according to the observed time-mean (Fig.3.21c). Tidal variations of $N^{2}$ were less pronounced than during the May SSF intrusion, and did not reduce stratification below one half of its time-mean value at any height. Numerical experiments show implementing tidal variations of $N^{2}$ does not change significantly the model predictions for the $M_{2}$ current ellipse.

Model solutions predict the velocity amplitudes in the upper water column well, if semidiurnal baroclinic pressure forcing is added to the model (Fig.3.26a,d, 27a,d). Numerical results display a current speed maximum near $z=20$ mab, followed by the adjustment to the free stream at heights $>25$ mab where the eddy viscosities reach their molecular value. For July 18-26, current amplitudes at heights $<50$ mab are slightly reduced by the addition of internal tide forcing (Fig.3.26a,d), while results for August 6-14 support the conclusion that the observed current maximum may be enhanced by internal tide shear (Fig.3.27a,d). Without current measurements between 12 and $30.5 \mathrm{mab}$, the observed velocity distribution in the bottom 
boundary layer is less clearly defined than during the SSF intrusion. Model-data comparison for May 6-14 suggests the transition between the turbulent boundary layer and upper water column is more gradual than indicated by the model (Fig.3.21a,d), a result that may also apply to the cases displayed in Fig.3.26 and Fig.3.27. Adding mean current shear representative of the observations does not increase the bottom boundary layer thickness for either time period (results not shown).

Numerical results for both periods suggest phase veering $\Delta \phi^{-} \approx-30^{\circ}$ and $\Delta \phi^{+} \approx 10^{\circ}$ in the friction-dominated bottom boundary layer $(z<25 \mathrm{mab})$, in good agreement with observation (Fig.3.26b,c;27b,c). As indicated by our measurements, the model predicts clockwise rotation of the major axis toward the bottom (Fig.3.26e,27e) and pronounced phase-lead of the near-bottom velocities (Fig.3.26f,27f). Comparison of numerical results with and without internal wave forcing shows phase angles are not affected by the mode-one baroclinic structure, indicating the internal wave amplitude is too small to have significant impact on the rotation of the velocity vector with depth.

Numerical bottom stress estimates for combined barotropic and baroclinic forcing are $\tau_{b_{M a j}}=1.4(2.0) \mathrm{cm}^{2} \mathrm{~s}^{-2}$ and $\tau_{b_{M i n}}=-0.6(-0.7) \mathrm{cm}^{2} \mathrm{~s}^{-2}$ for July 18-26 (August 6-14). In comparison, the observational values are $\tau_{b_{M a j}}=$ $1.6 \pm 0.1(2.2 \pm 0.3) \mathrm{cm}^{2} \mathrm{~s}^{-2}$ and $\tau_{b_{\text {Min }}}=-0.7 \pm 0.1(-1.0 \pm 0.2) \mathrm{cm}^{2} \mathrm{~s}^{-2}$. Doubling the mixed layer height from 3 to 6 mab enhances the numerical bottom stress magnitudes by about $10 \%$.

\subsection{Summary and Conclusions}

- Linear regression coefficients between the burst-averaged BASS current measurements at $0.26-2.56 \mathrm{mab}$ and $\log (z)$ are $>0.95$ for more than $87 \%$ of the summer deployment from July 12-August 14, compared to $79 \%$ for the winter analysis period February 11-March 11 (WB). Thus, the velocity distribution in the lowest $\approx 2.5 \mathrm{~m}$ closely follows the logarithmic law of the wall throughout the year, despite the fact that the near-bottom waters are stratified in summer (thermal stratification $<0.010 \pm 0.001^{\circ} \mathrm{C}$ for $z<2.5 \mathrm{~m}$ ) and homogeneous in winter. Best-fit logarithmic profiles to acoustic current meter data between 0.26-2.56 mab 
give $u_{*}=1.1 \pm 0.4 \mathrm{~cm} \mathrm{~s}^{-1}$ for the summer deployment, in good agreement with $u_{*}=1.2 \pm 0.4 \mathrm{~cm} \mathrm{~s}^{-1}$ for the winter period (WB). Least-squares fits of the instantaneous bottom stress to current speed squared yields quadratic drag coefficients that are very similar for winter and summer (Table 3.1). The lack of variation in $c_{D}$ implies that accurate stress estimates may be obtained from the quadratic drag law at times when logarithmic fits cannot be made.

- A SSF intrusion took place in May, advecting warm, saline water from the southeast of ST1 into the shallower regions to the northwest. The SSF intrusion was bottom-trapped from May 6-14, and extended to the surface between May 14-22. Analysis of buoyancy frequency estimates indicates vertical stratification during the SSF intrusion was a strong function of flow direction: below the point where the SSF intersects ST1, $N^{2}$ was largest on-bank of the mooring location. The strongly stratified near-bottom waters were advected off-bank during ebb, resulting in peak buoyancy frequencies at the reversal from ebb to flood. Above the intersection point with the SSF, vertical stratification was largest off-bank of ST1, and the largest vertical stratification occured at the reversal from flood to ebb. Richardson numbers show strong dependence on the variation of $N^{2}$, indicating turbulence production in the bottom boundary layer varied significantly during the course of a tidal cycle.

- Observational analysis of the May SSF intrusion indicates subtidal velocity shear contributes significantly to turbulence production in the BBL at the end of ebb/ beginning of flood. During this phase of the tidal cycle, the subtidal and tidal currents reinforce each other to enhance current shear, while the opposite effect takes place at the end of flood/beginning of ebb. Below the intersection point of the SSF with ST1, stratification is weak at the reversal from flood to ebb when the subtidal currents oppose the tidal flow. Hence, the impact of reduced current shear on turbulence production is limited. The subtidal currents act mainly to enhance mixing near the bottom around the reversal from ebb to flood when stratification is largest, thereby introducing an asymmetry that has to be considered in conceptual and numerical models of the bottom boundary layer. 
- Analysis of the observed $M_{2}$ tidal currents during the May SSF intrusion shows stratification causes a current speed maximum in the lower water column, in agreement with earlier observations by Maas and van Haren (1987). Below the maximum, observations display stronger rotation of the major axis and phase-shift of the velocity vector toward the bottom than during nearly homogeneous winter conditions. This behavior indicates stratification limits the height over which bottom friction is balanced. Amplitudes $R^{ \pm}$and veering angles $\Delta \phi^{ \pm}$representative of the surfaceintensified phase show buoyancy forcing affects the anticlockwise rotary component to a lesser degree than the clockwise component, thus reflecting the different scales for the clockwise and anticlockwise boundary layers (Souza and Simpson, 1996; WB).

- Numerical results from a one-dimensional model using the MY2.5 turbulence closure scheme predict the top of the turbulent boundary layer is marked by a pronounced current speed maximum near $12 \mathrm{mab}$, followed by abrupt adjustment to the frictionless interior. This is in disagreement with our observations which suggest a maximum of smaller magnitude near $18 \mathrm{mab}$ or higher and a more gradual transition to the free-stream. Implementing mean velocity shear representative of the observations does not change the model solutions for the $M_{2}$ current distribution. This result opposes indications from observational analysis suggesting subtidal flow shear enhances turbulence production near the bottom if the tidal current is aligned with the mean along-bank circulation.

Model results for the surface intensified phase predict the observed $M_{2}$ currents and estimated bottom stresses well, but do underestimate the vertical extent of the region with significant velocity shear by about $10 \mathrm{~m}$. Due to enhanced mixing coefficients when stratification is small, the average thickness of the tidal boundary layer is about $5 \mathrm{~m}$ larger if $N^{2}$ varies on tidal time scales than if vertical stratification is fixed according to the observed time-mean. At the same time, a more gradual transition from the turbulent boundary layer to the free-stream is achieved in better agreement with observation. Similar to results for May 6-14, adding mean flow shear to the semidiurnal pressure forcing does not significantly improve the model results for the bottom boundary layer thickness. 
- Tidal analysis of velocity data shows baroclinic structures in summer which resemble mode-one internal tides at the $M_{2}$ frequency. The relative phase of the barotropic and internal tides changes with time such that the corresponding velocities oppose or reinforce each other near the surface, while the opposite behavior occurs below the point of baroclinic flow reversal. Similar to the May SSF event, phase and inclination angles display strong veering at heights $<30$ mab. The presence of mode-one internal tides is supported by numerical results from the MY2.5 model.

- The overall performance of the one-dimensional numerical model considered here is limited by the under-estimation of the bottom boundary layer thickness and the abrupt adjustment between the turbulent region and the free-stream, particularly if near-bottom stratification is large. Similar conclusions were reached for nearly homogeneous winter conditions, when stratification was more than one order of magnitude weaker than during the periods investigated in this study (WB). Simpson et al. (1996) found that lower versions of the MY model with prescribed turbulent length scale distribution (MY 2.2, MY 2.0) fail to predict the correct level of dissipation in the strongly stratified portion of the water column. Their conclusion was that a mid-water source of turbulent kinetic energy exists which is not represented in the numerical models. On Georges Bank, such a source may be given by stirring and mixing due to internal wave solitons. In our data, we observe solitary waves during strongly stratified conditions, but find no evidence of their presence if stratification is weak. Since similar model-data discrepancies were observed for both weakly and strongly stratified times, we conclude the MY2.5 turbulence closure may under-predict turbulence production in the presence of stratification. As noted by Polzin (1996), turbulent mixing in the ocean can occur over a range of Richardson numbers, which includes $0.25<R i<1.0$. The transition from molecular to turbulent mixing takes place abruptly near $R i=0.25$ in the MY2.5 model, which may help explain the data-model difference found here. It is also possible that the vertical boundary layer structure over Georges Bank cannot be modeled adequately with a one-dimensional model. In particular, the $M_{2}$ current profile at ST1 may not be described by its response to a specified density field alone, but may partly be determined by the flow structure on- and off-bank of the mooring location. Future work will address this question. 


\section{Appendix}

For frictionless flow, the basic equations governing internal wave motion are

$$
\begin{aligned}
& \frac{\partial u}{\partial x}+\frac{\partial v}{\partial y}+\frac{\partial w}{\partial z}=0 \\
& \frac{\partial u}{\partial t}-f v=-\frac{1}{\rho_{0}} \frac{\partial p}{\partial x} \\
& \frac{\partial v}{\partial t}+f u=-\frac{1}{\rho_{0}} \frac{\partial p}{\partial y} \\
& \frac{\partial w}{\partial t}=-\frac{1}{\rho_{0}} \frac{\partial p}{\partial z}-\frac{\rho g}{\rho_{0}} \\
& \frac{\partial \rho}{\partial t}-\frac{\rho_{0} N^{2}}{g} w=0
\end{aligned}
$$

where $w$ is the vertical velocity. (A.1d) combines with (A.1e) to form the expression

$$
\frac{1}{\rho_{0}} \frac{\partial^{2} p}{\partial z \partial t}=-w N^{2}-\frac{\partial^{2} w}{\partial t^{2}}
$$

Substituting (A.1a-c) into (A.2) and solving for $w$ gives

$$
\frac{\partial^{2}}{\partial t^{2}}\left(\frac{\partial^{2}}{\partial x^{2}}+\frac{\partial^{2}}{\partial y^{2}}+\frac{\partial^{2}}{\partial z^{2}}\right) w+f^{2} \frac{\partial^{2} w}{\partial z^{2}}+N^{2}\left(\frac{\partial^{2}}{\partial x^{2}}+\frac{\partial^{2}}{\partial y^{2}}\right) w=0
$$

Assuming a wavelike solution in $x$ and no along-bank propagation $\left(\frac{\partial}{\partial y}=0\right)$, we define $w_{n}=A_{n} \Phi_{n}(z) e^{i\left(k_{n} x-\sigma t\right)}$, where $A_{n}$ is amplitude, $\Phi_{n}(z)$ is a vertical structure function, $k_{n}$ is wave number, and the subscript $\mathrm{n}=0,1,2, \ldots$ refers to the vertical normal modes. Substitution in (A.3) yields the eigenvalue problem

$$
\frac{\partial^{2}}{\partial z^{2}} \Phi_{n}+m_{n}^{2} \Phi_{n}=0
$$

where

$$
m_{n}^{2}=k_{n}^{2} \frac{\frac{N^{2}}{\sigma^{2}}-1}{1-\frac{f^{2}}{\sigma^{2}}}
$$


The surface $(z=H)$ and bottom $(z=0)$ boundary conditions to (A.4) are $\Phi(H)=$ $\Phi(0)=0$. To find the amplitude $A_{n}$, we integrate the $x$-derivative of (A.2) over depth and obtain

$$
\frac{\partial P_{n}}{\partial x}=\frac{k_{n}}{\sigma} A_{n} \int_{z}^{H}\left(\sigma^{2}-N^{2}\right) \Phi_{n}\left(z^{\prime}\right) d z^{\prime}+\left[\frac{\partial P_{n}}{\partial x}\right]_{H},
$$

where we substituted $\frac{\partial p_{n}}{\partial x}=\frac{\partial P_{n}}{\partial x} e^{i\left(k_{n} x-\sigma t\right)}$. In (A.6), the subscript $H$ denotes the pressure gradient evaluated at the surface. For $\mathrm{n}=1,2,3, \ldots$, the integral $\int_{0}^{H} \frac{\partial P_{n}}{\partial x} d z=0$, so that the constant $A_{n}$ can be determined from

$$
A_{n}=-\left[\frac{\partial P_{n}}{\partial x}\right]_{B} \frac{\sigma}{k_{n}} \frac{H}{\int_{0}^{H}\left[\int_{z}^{H}\left(\sigma^{2}-N^{2}\right) \Phi_{n}\left(z^{\prime}\right) d z^{\prime}\right] d z} .
$$

With $\left[\frac{\partial P_{1}}{\partial x}\right]_{H}=i\left(1-\frac{f^{2}}{\sigma}\right)\left[U_{1}\right]_{H}$, where $\left[U_{1}\right]_{H}$ is the surface amplitude of the modeone cross-bank velocity, the mode-one surface pressure gradient may be estimated from the measured velocity profiles. The model forcing is given by the sum of the barotropic $(n=0)$ and first baroclinic $(n=1)$ pressure gradients

$$
\frac{\partial p}{\partial x}=\left[\frac{\partial P_{0}}{\partial x}+\frac{\partial P_{1}(z)}{\partial x} e^{i \beta}\right] e^{-i \sigma t}
$$

where $\beta$ is the relative phase between the barotropic and baroclinic tides.

Acknowledgments: We thank Sandy Williams and his co-workers for collecting and making the BASS tripod data available, Janet Fredericks, Jim Churchill and Tom Gross for their assistance with BASS data processing, Steve Lentz, Bob Weller, Jim Manning and their co-workers for obtaining the moored meteorological and oceanographic data at ST1 and ST2 and making the data available, Nancy Brink for processing the moored data, Carol Alessi for her help with managing the data set, and Anne-Marie Michael for typing large parts of this paper. Julio Candela provided the version of the Godin harmonic analysis code used in this study. The MY2.5 1-D numerical circulation model was provided by Steve Monismith and is based on the Blumberg-Mellor 3-D hydrodynamic circulation model (Blumberg 
and Mellor, 1987). We especially want to thank John Trowbridge and Steve Lentz for many helpful discussions and advice about all aspects of this study. This work was supported by the National Science Foundation under grants OCE 93-13671 and OCE 96-32357 as part of the U.S. GLOBEC/Georges Bank Program. 


\section{References}

Blumberg, A. F., and G. L. Mellor, A description of a three-dimensional coastal ocean circulation model. Coastal and Estuarine Science, 4, 1-16, 1987.

Flagg, C. N., Hydrographic structure and variability. In: Georges Bank, R. H. Backus, editor, The MIT Press, 108-124, 1987.

Foreman, M. G. G., Manual for tidal currents analysis and prediction, Pacific Marine Science Report 87-6, Institute of Ocean Science, Patricia Bay, Sidney, B.C, 1978.

Kagan, B. A., On the structure of the tidal flow in the sea. Izv. Atmos. and Oceanic Phys., 2, 575-581, 1966 (English translation).

Kundu P. K., In: Fluid Mechanics, Academic Press Inc., San Diego, California, $463-465 \mathrm{~g}, 1990$.

Maas, L. R. M., and J. J. M. van Haren, Observations on the vertical structure of tidal and inertial currents in the central North Sea. J. Marine Res., 45, 293-318, 1987.

Mellor, G. L., and T. Yamada, A hierarchy of turbulence closure models for planetary boundary layers. J. Atmos. Sc., 33, 1791-1896, 1974.

Mellor, G. L., and T. Yamada, Development of a turbulence closure model for geophysical fluid problems. Rev. Geophys. Space Physics, 20, 851-875, 1982.

Polzin, K., Statistics of the Richardson Number: Mixing Models and Finestructure. J. Physical Oceanogr.,26, 1409-1425, 1996.

Prandle, D., The vertical structure of tidal currents. Geophys. Astrophys. Fl. Dynamics, 22, 29-49, 1982 a.

Prandle, D., The vertical structure of tidal currents and other oscillatory flows. Continental Shelf Res., 1 (2), 191-207, $1982 \mathrm{~b}$.

Simpson, J. H., W. Crawford, T. P. Rippeth, A. R. Campbell and J.V.S. Cheok, The vertical structure of turbulent dissipation in shelf seas. J. Physical Oceanogr., 26, 1579-1590, 1996. 
Soulsby, R. L., The bottom boundary layer of shelf seas. Physical Oceanogr. of Coastal and Shelf Seas, B. Johns, editor, Elsevier, Amsterdam, pp. 189-266, 1983.

Soulsby, R. L., Tidal-current boundary layers. The Sea, 9, 523-566, 1990.

Souza, A. J., and J. H. Simpson, The modification of tidal ellipses by stratification in the Rhine ROFI. Continental Shelf Res., 16, 997-1007, 1996.

Stacey, M. T., S. G. Monismith, and J. R. Bureau, Observations of turbulence in a partially stratified estuary. Submitted to J. Physical Oceanogr..

Sverdrup, H. U., Dynamics of tides on the north Siberian shelf. Geofys. Publ. Norske Videns Akad., 4, Oslo, Norway, 1927.

Visser, A. W., A. J. Souza, K. Hesner, and J. H. Simpson, The effect of stratification on tidal current profiles in a region of freshwater influence. Oceanologica acta, 17, 369-382, 1994.

Werner, S. R., The vertical structure of the bottom boundary layer on the southern flank of Georges Bank during late winter. M.S. Thesis, Joint Program in Oceanography/ Applied Ocean Science and Engineering, Woods Hole Oceanographic Institution, Woods Hole, Mass, Massachusetts Institute of Technology, Cambridge, Mass, 1996. 


\begin{tabular}{c|c|c|c|c|c}
\hline \multicolumn{3}{c|}{ Feb 11-Mar 11 } & \multicolumn{3}{c}{ Jul 12-Aug 14 } \\
\hline $\begin{array}{c}\text { Height } \\
{[\mathbf{m a b}]}\end{array}$ & $\begin{array}{c}\mathbf{C}_{\mathbf{D}} \\
\mathbf{1 0}^{-\mathbf{3}}\end{array}$ & $\begin{array}{c}\boldsymbol{\Delta} \mathrm{C}_{\mathbf{D}} \\
\mathbf{1 0}\end{array}$ & $\begin{array}{c}\text { Height } \\
{[\text { mab] }}\end{array}$ & $\begin{array}{c}\mathrm{C}_{\mathbf{D}} \\
\mathbf{1 0}^{-\mathbf{3}}\end{array}$ & $\begin{array}{c}\boldsymbol{\Delta} \mathbf{C}_{\mathbf{D}} \\
\mathbf{1 0}^{-\mathbf{3}}\end{array}$ \\
\hline 0.22 & 3.62 & 0.26 & 0.26 & 4.29 & 0.12 \\
0.58 & 3.14 & 0.18 & 0.61 & 3.48 & 0.10 \\
1.18 & 2.62 & 0.14 & 1.21 & 2.83 & 0.07 \\
2.53 & 2.21 & 0.10 & 2.56 & 2.39 & 0.05 \\
4.43 & 1.78 & 0.10 & 4.46 & 1.95 & 0.05 \\
6.00 & 1.66 & 0.10 & 6.00 & 1.68 & 0.05 \\
\hline
\end{tabular}

Table 3.1: Quadratic bottom drag coefficients $c_{D}$ for winter and summer computed from leastsquares fits of $u_{*}^{2}$ versus $U^{2}$ at the BASS acoustic current sensors and the lowest VMCM, and $95 \%$ confidence limits $\Delta c_{D}$ of the least-squares fits. 




Fig.3.1: Bathymetry (in meters) of Georges Bank and adjacent region, approximate location of the Tidal Mixing Front (TMF) and Shelf-Slope Front (SSF), and the GLOBEC Stratification Study mooring sites ST1 and ST2. The $+x$ direction is on-bank $\left(330^{\circ} \mathrm{T}\right)$. 




Fig.3.2: Schematic of the GLOBEC array at ST1 and ST2. 




Fig.3.3: Temperature difference $\Delta T$ between BASS thermistors 5 and 2 at 2.5 and 0.6 mab. 



Fig.3.4: (a) Time series of estimated friction velocity $u_{*}$, and (b) diagram of $\frac{\Delta u_{*}^{95}}{u_{*}}$ versus $u_{*}$. In (a), missing values $u_{*}$ are filled in by linear interpolation. 


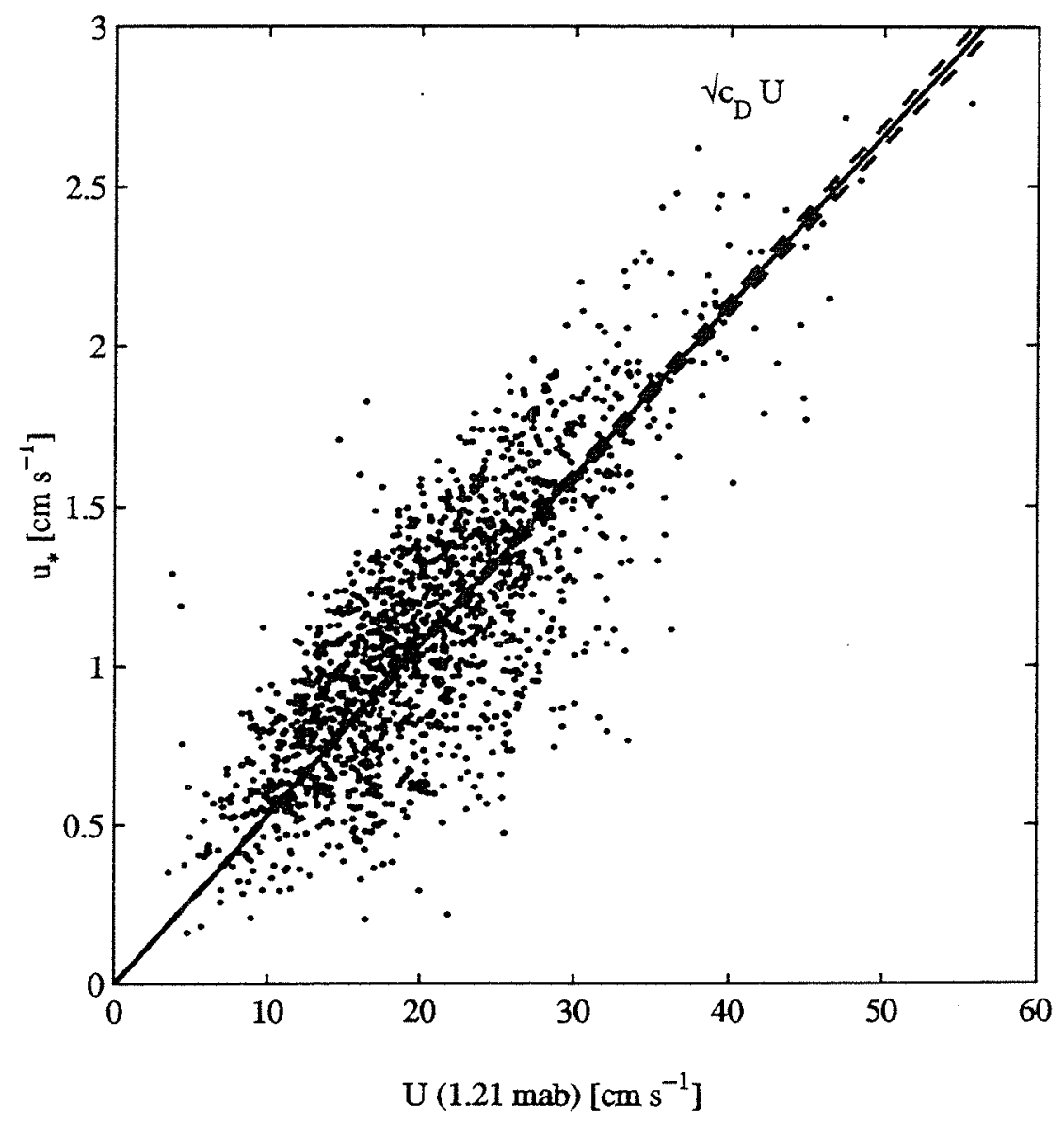

Fig.3.5: Estimated friction velocity $u_{*}$ versus measured speed $U$ at 1.21 mab (BASS pod 3). The solid line depicts the slope $\sqrt{c_{D}} \cdot U$ with $c_{D}$ obtained from least squares fits of $c_{D} U^{2}$ to $u_{*}^{2}$. Dashed lines give the $95 \%$ confidence limits of the fitted slope. 
(a)

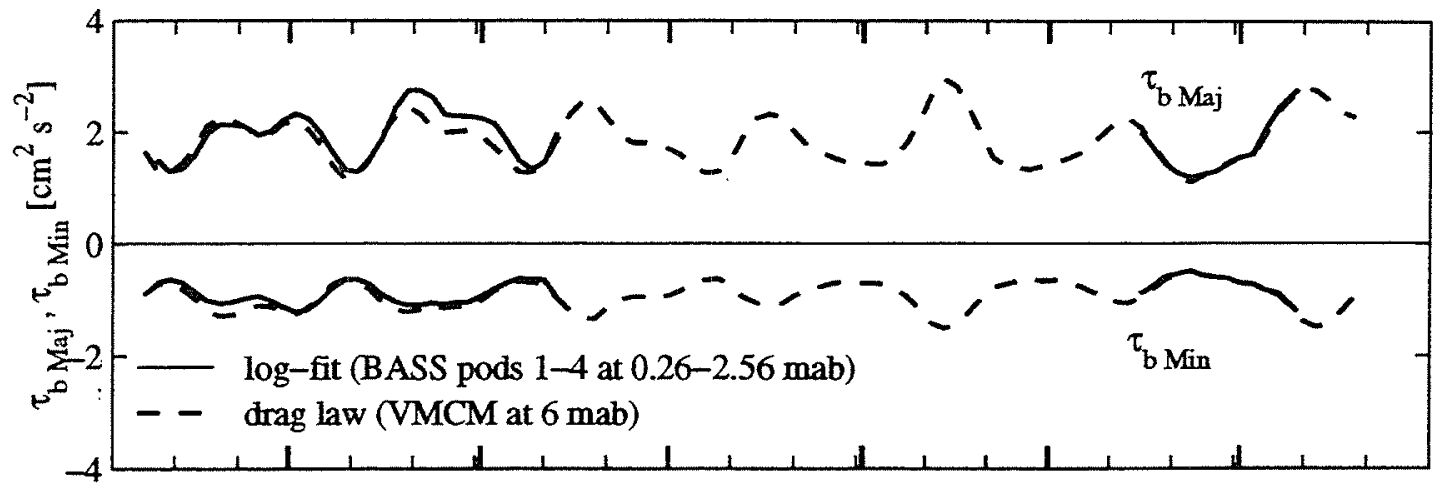

(b)


Fig.3.6: (a) Tidal $\left(M_{2}\right)$ and (b) subtidal (time-scales $>33 \mathrm{hrs}$ ) estimates of kinematic bottom stress obtained from (solid) least-squares logarithmic fits to BASS velocity measurements at $0.26-$ $2.56 \mathrm{mab}$ and (dashed) the quadratic drag law at $6 \mathrm{mab}$. 
(a)



(b)

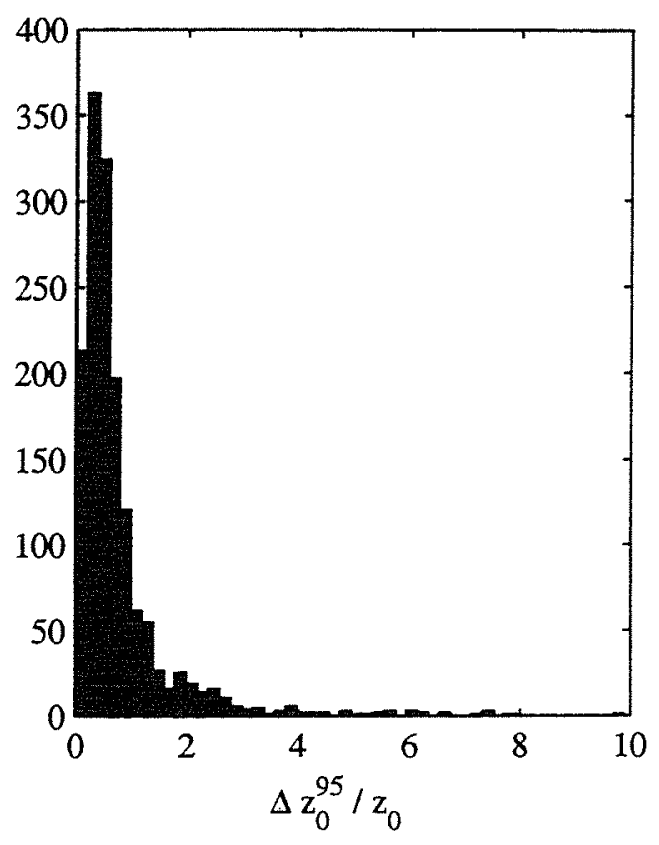

Fig.3.7: Histograms of (a) bottom roughness $\log _{10}\left(z_{0}[\mathrm{~mm}]\right)$ and (b) $\frac{\Delta z_{0}^{95}}{z_{0}}$. 

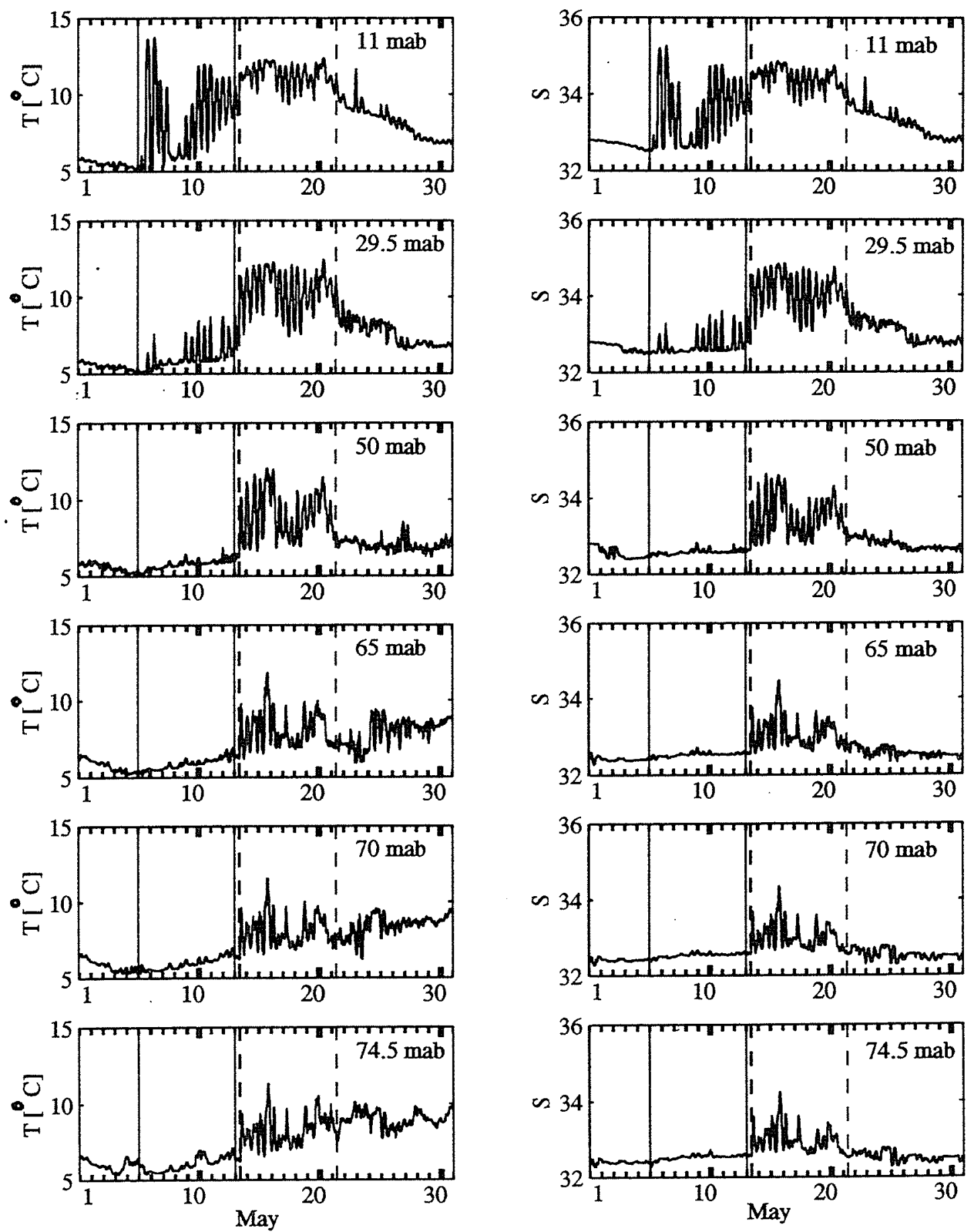

Fig.3.8: Time series of hourly averaged SeaCAT (left) temperature and (right) salinity data at ST1 during the May SSF intrusion event. Solid and dashed vertical lines mark the bottom-trapped phase May 6-14 and the surface-intensified phase May 14-22, respectively. 

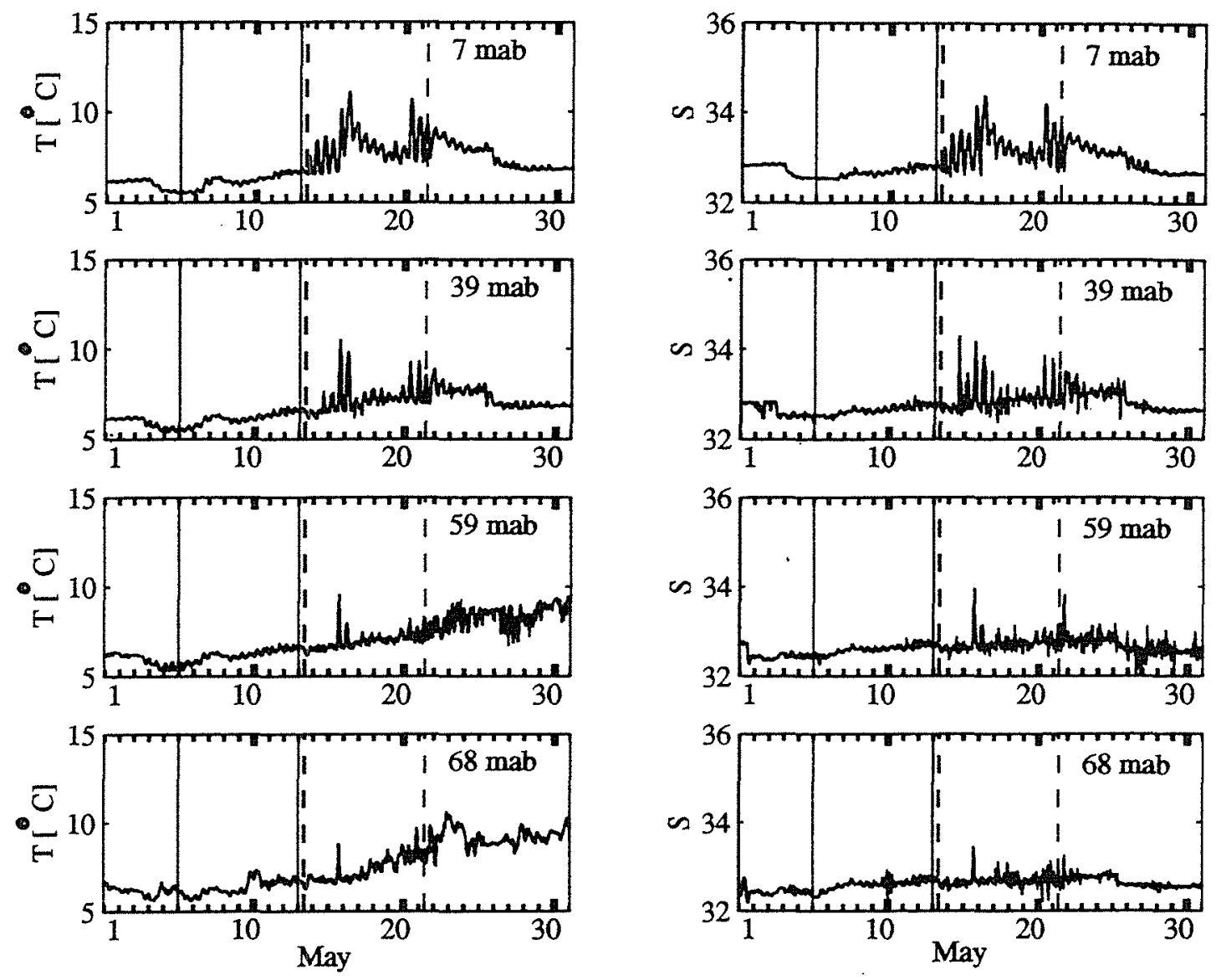

Fig.3.9: Time series of hourly averaged SeaCAT (left) temperature and (right) salinity data at ST2 during the May SSF intrusion event. Solid and dashed vertical lines are the same as in Fig.3.8. 

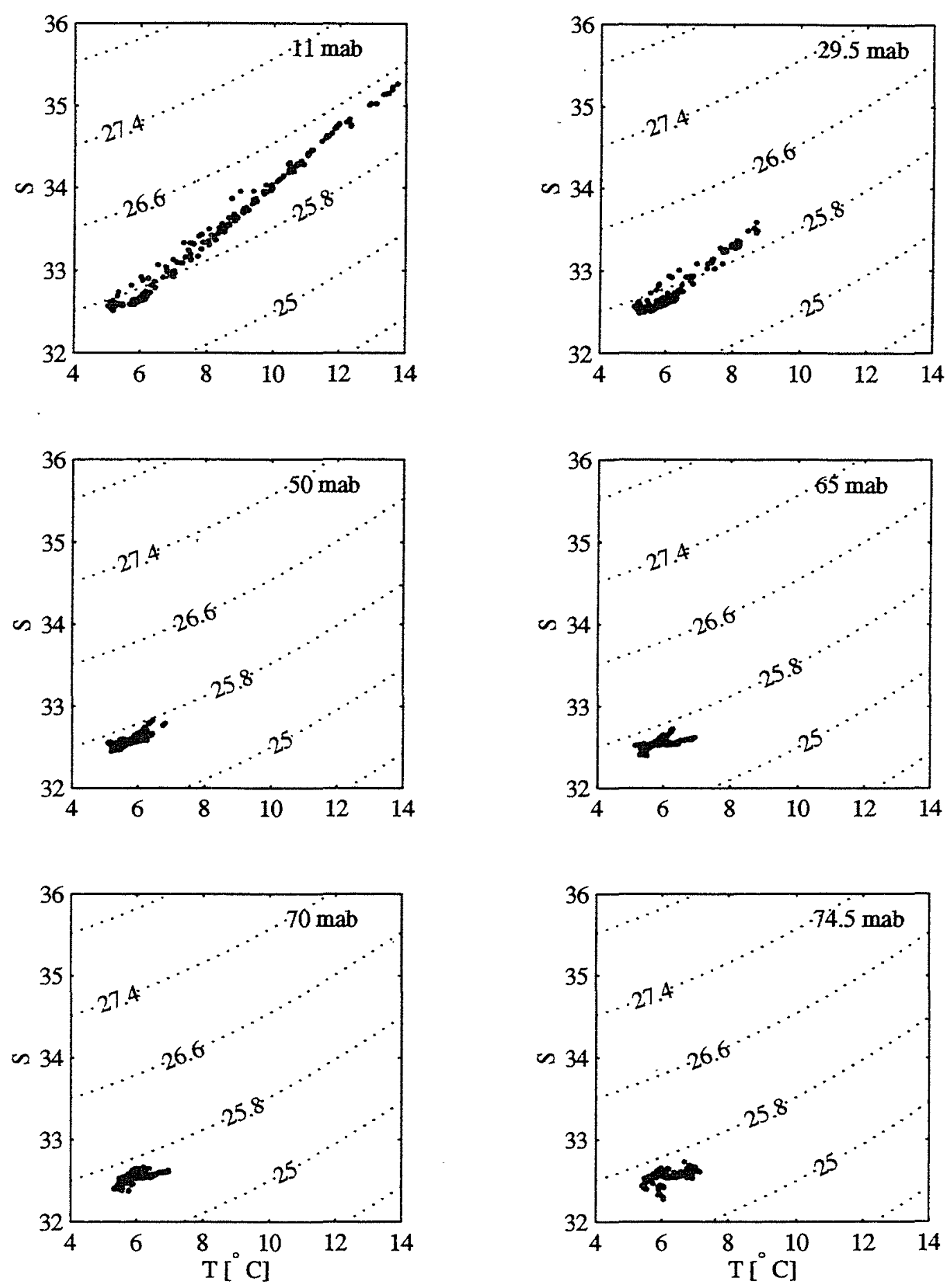

Fig.3.10: Temperature-Salinity $(T-S)$ diagrams for May 6-14 from hourly-averaged SeaCAT data. Dotted lines are lines of constant $\sigma_{\theta}$. 
(a)

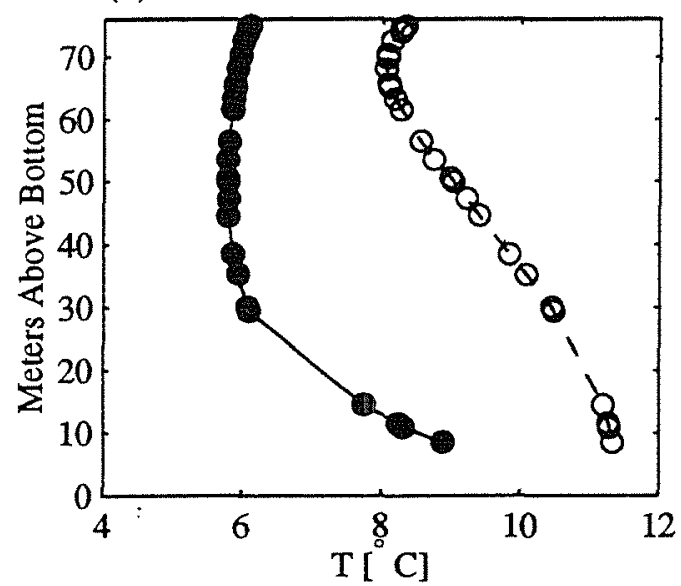

(c)



(b)

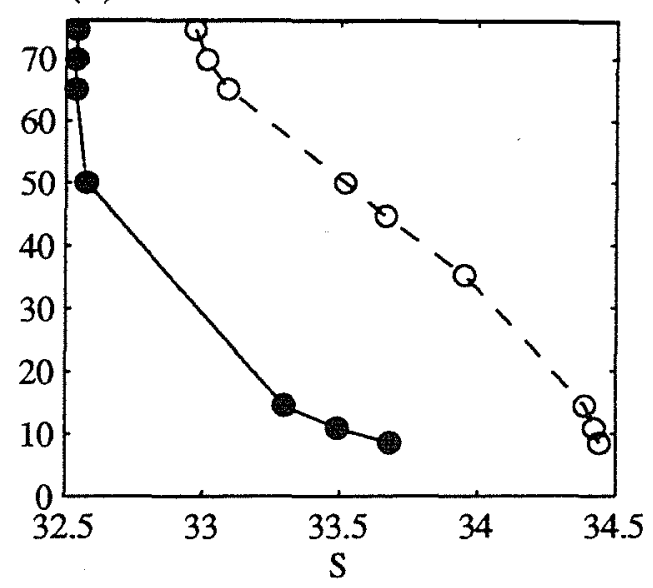

(d)

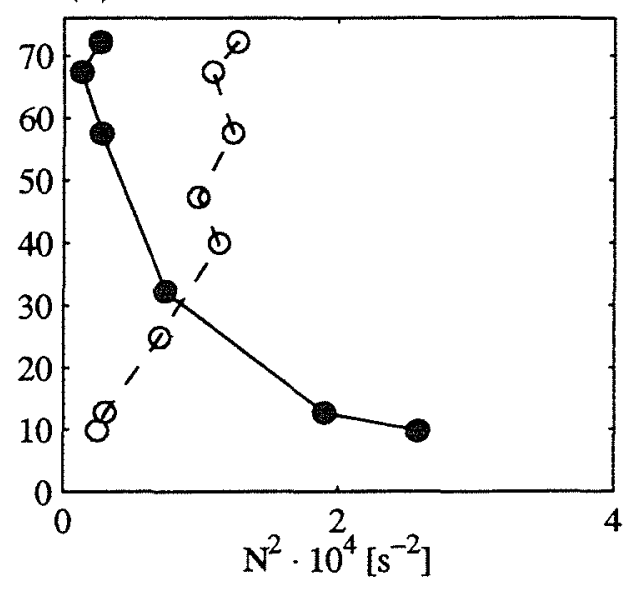

Fig.3.11: Profiles of time-mean (a) temperature $\mathrm{T}$, (b) salinity $\mathrm{S}$, (c) potential density $\sigma_{\theta}$, and (d) buoyancy frequency squared $N^{2}$ for (solid) May 6-14 and (dashed) May 14-22. 
(a)

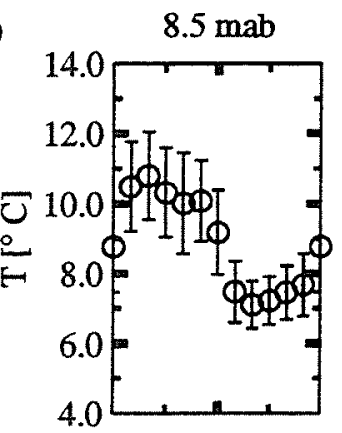

(b)

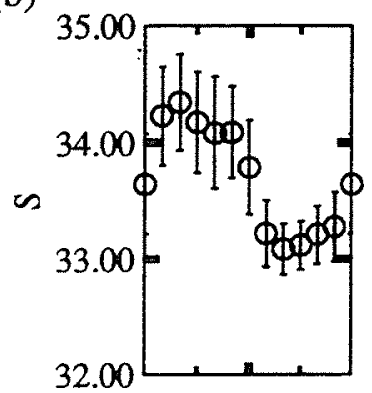

(c)

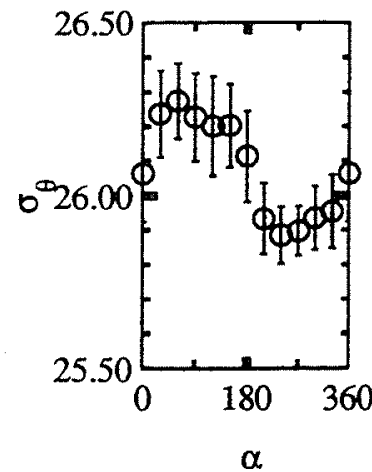

$11 \mathrm{mab}$
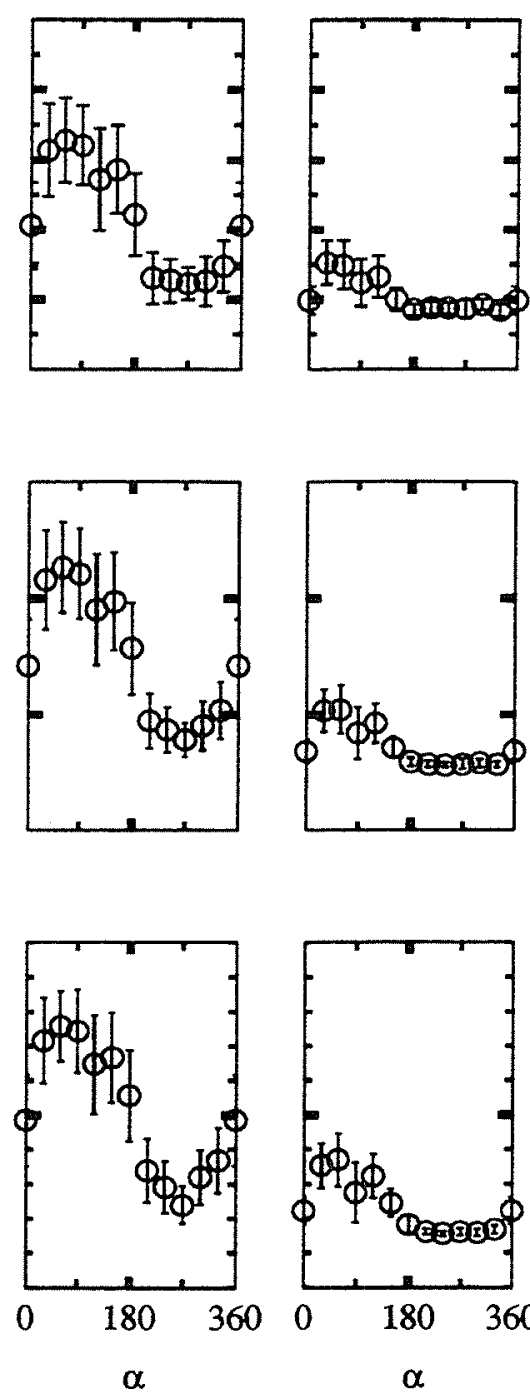

$29.5 \mathrm{mab}$
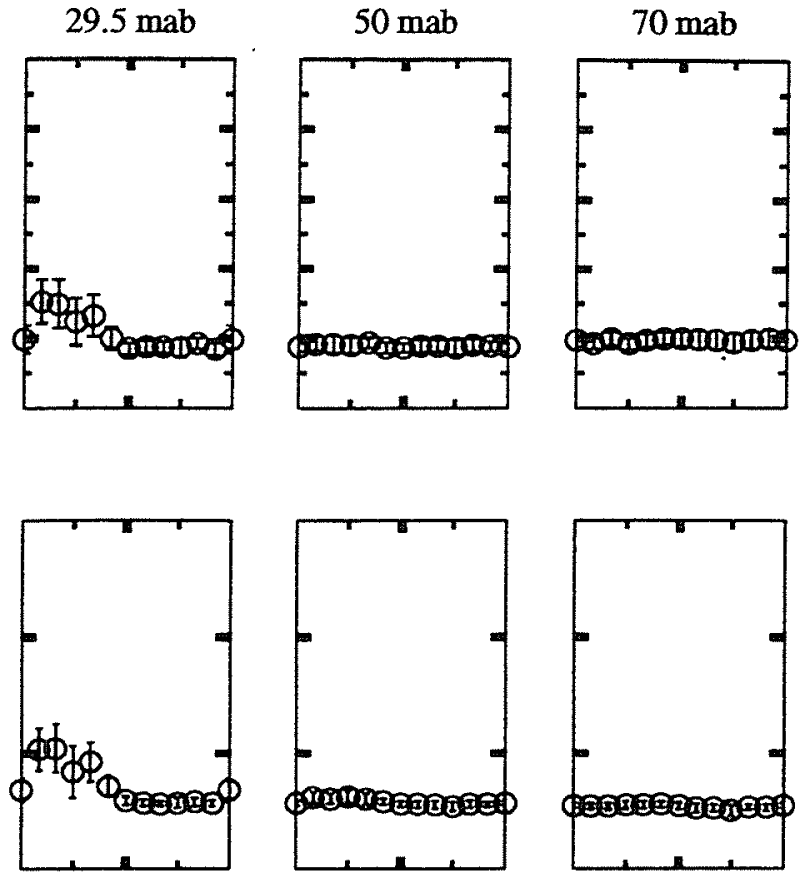

$70 \mathrm{mab}$








Fig.3.13: Schematic of the $M_{2}$ tidal ellipse. The flow direction $\alpha$ is measured clockwise from the $+x$ direction (Fig.3.1), such that flood (ebb) currents correspond to $\alpha=0^{\circ}\left(180^{\circ}\right)$. 

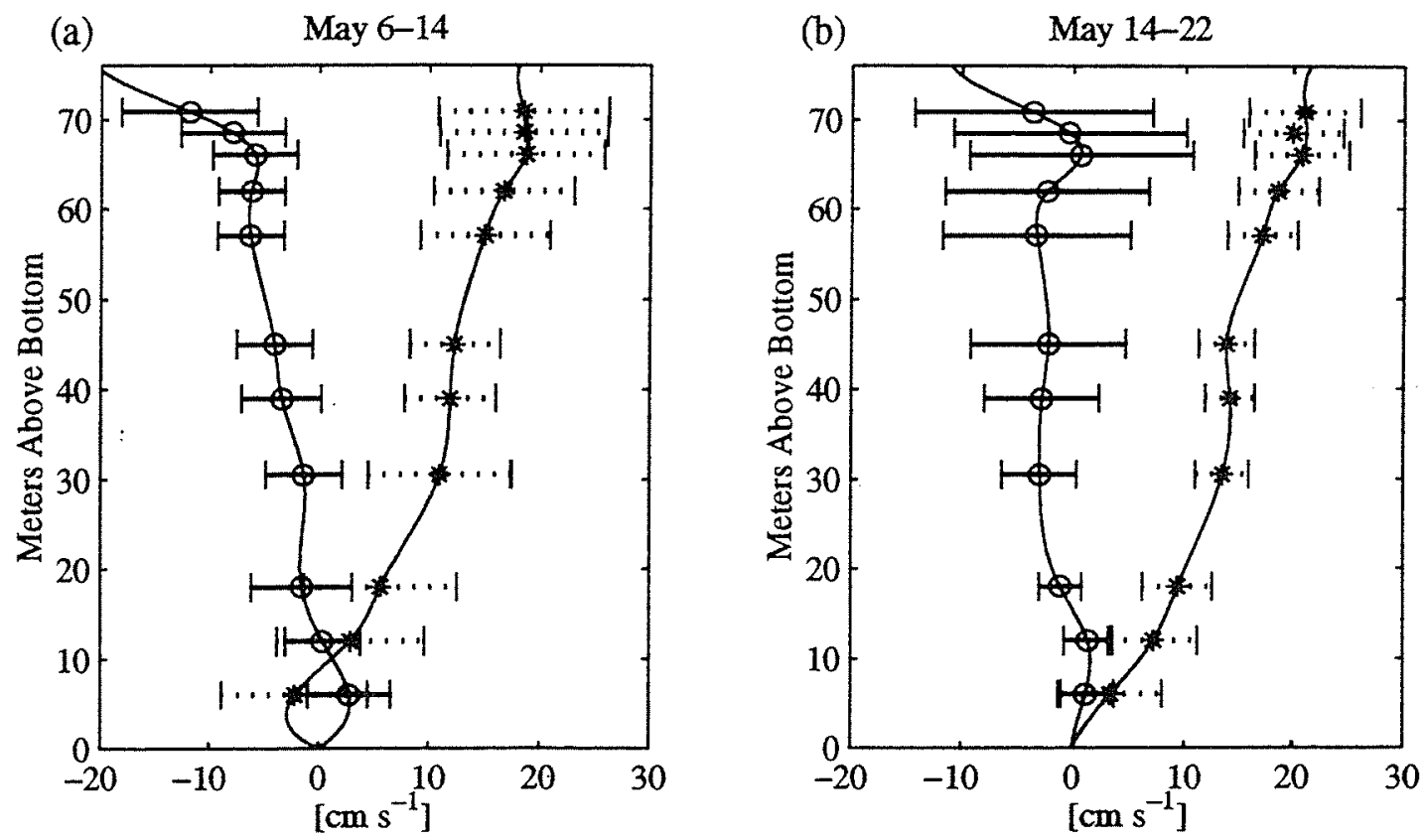

Fig.3.14: Profiles of the subtidal current velocities (circles) $u_{0}$ and (asterisks) $v_{0}$ for (a) May 614 and (b) May 14-22 from hourly-averaged VMCM data. Horizontal bars denote the deviation from the mean at the $95 \%$ confidence level. Solid lines correspond to velocity profiles entered in the numerical model. 
Fig.3.15: Vertical (a) temperature gradients $\frac{\Delta T}{\Delta z}$, (b) salinity gradients $\frac{\Delta S}{\Delta z}$, (c) buoyancy frequency squared $N^{2}$, and (d) percentage of events $R i<0.5$ based on the (circles) total and (diamonds) high-passed (temporal variations < 33 hrs) current shear for May 6-14 as a function of the depth-dependent tidal flow direction $\alpha$ defined in Figs.3.12 and 3.13. Results are from hourly-averaged data. Error bars in (a)-(c) denote the standard error at the $95 \%$ significance level. Error limits in (d) were obtained from Monte Carlo simulation (see text). 



(c)
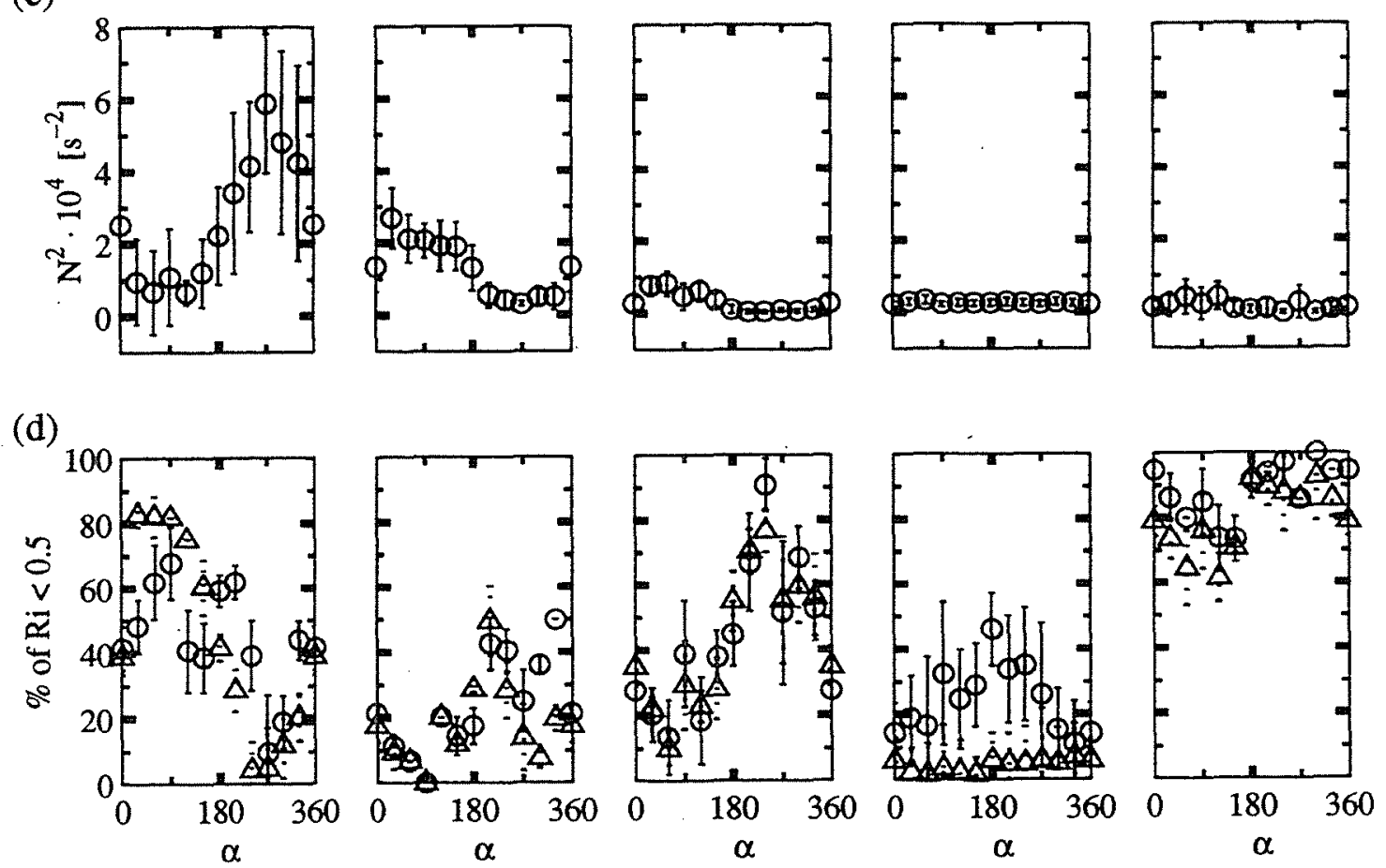

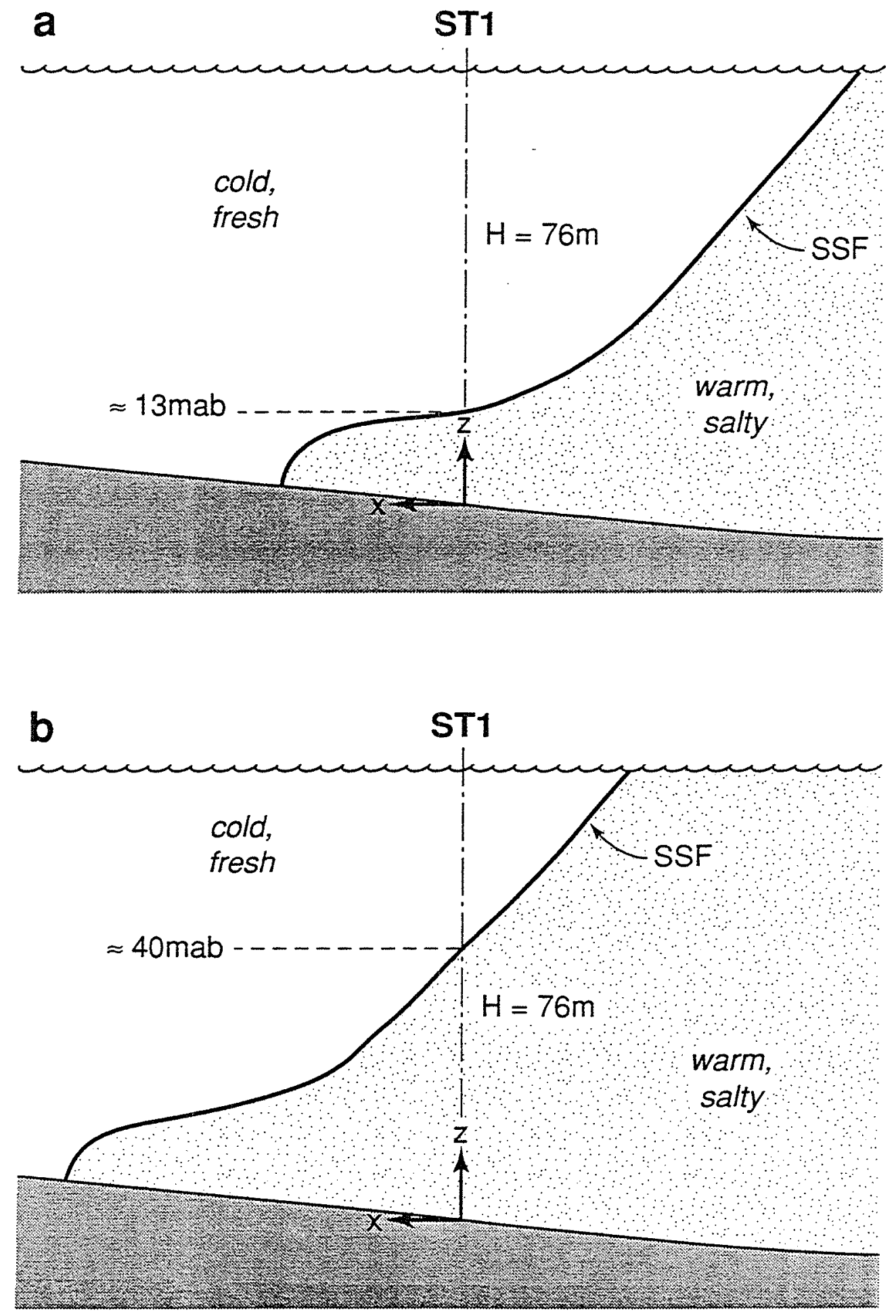

Fig.3.16: Schematic of the May SSF intrusion during the (a) bottom-trapped phase (May 6-14) and (b) surface-intensified phase (May 14-22). 

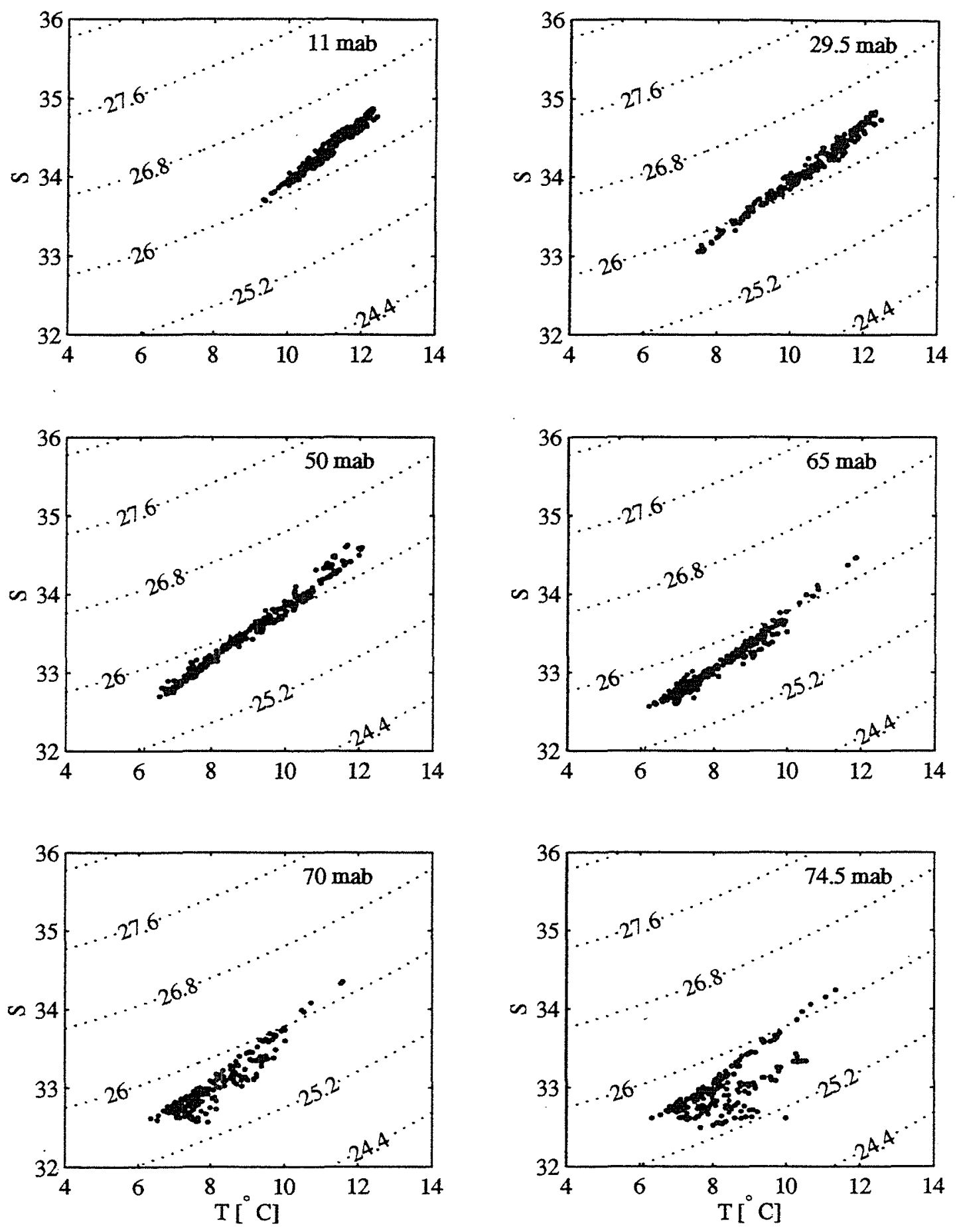

Fig.3.17: Temperature-Salinity $(T-S)$ diagrams for May 14-22 from hourly-averaged SeaCAT data. Dotted lines are lines of constant $\sigma_{\theta}$. 
(a)

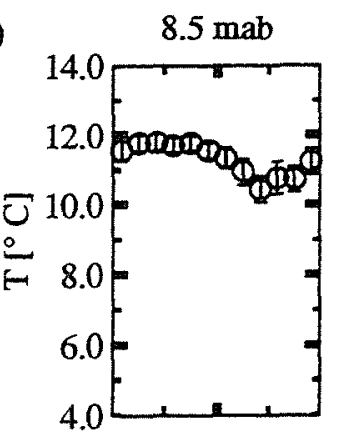

(b)



(c)

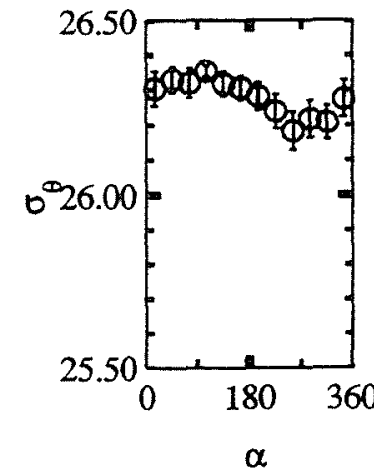


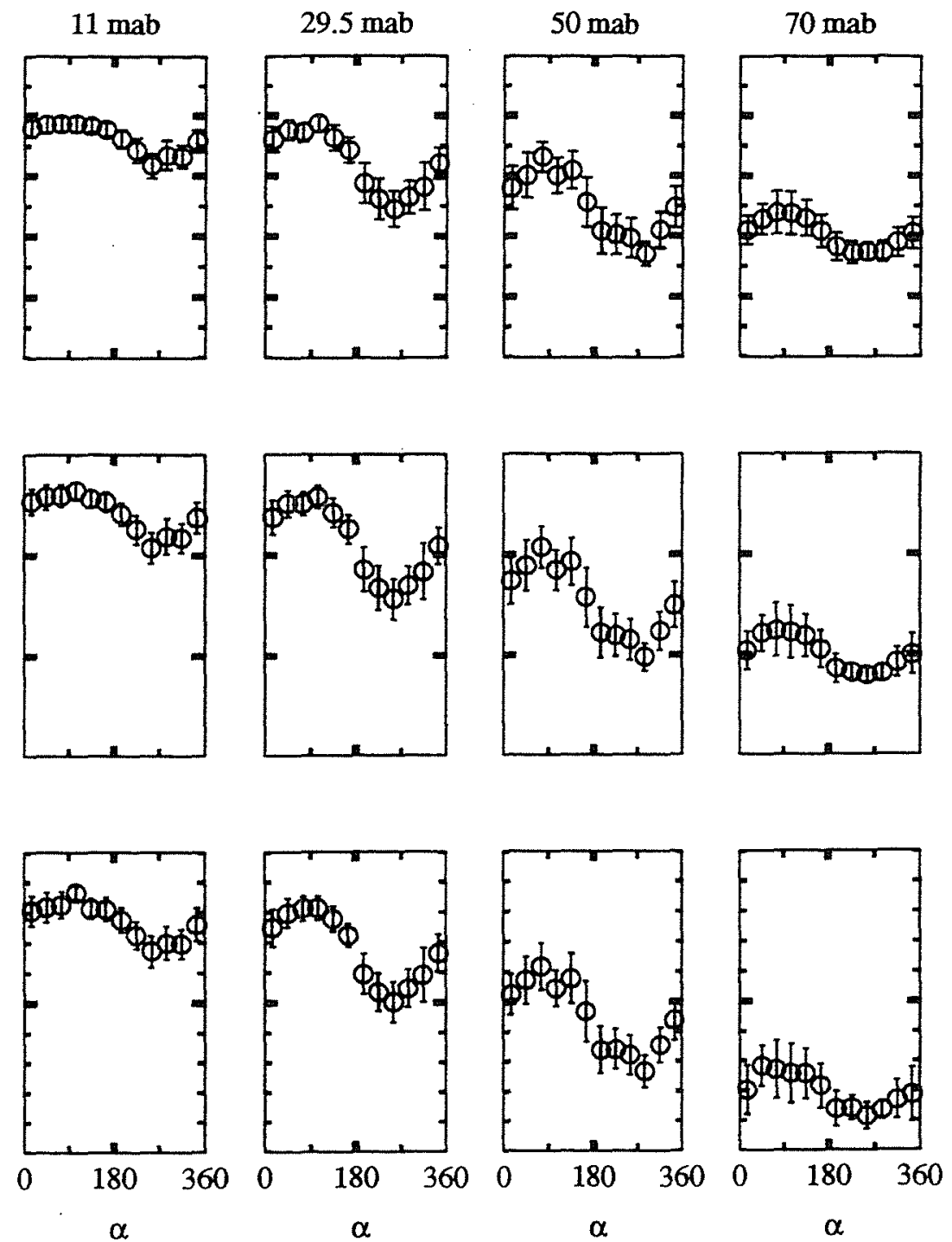

Fig.3.18: Same as Fig.3.12, but for May 14-22. 

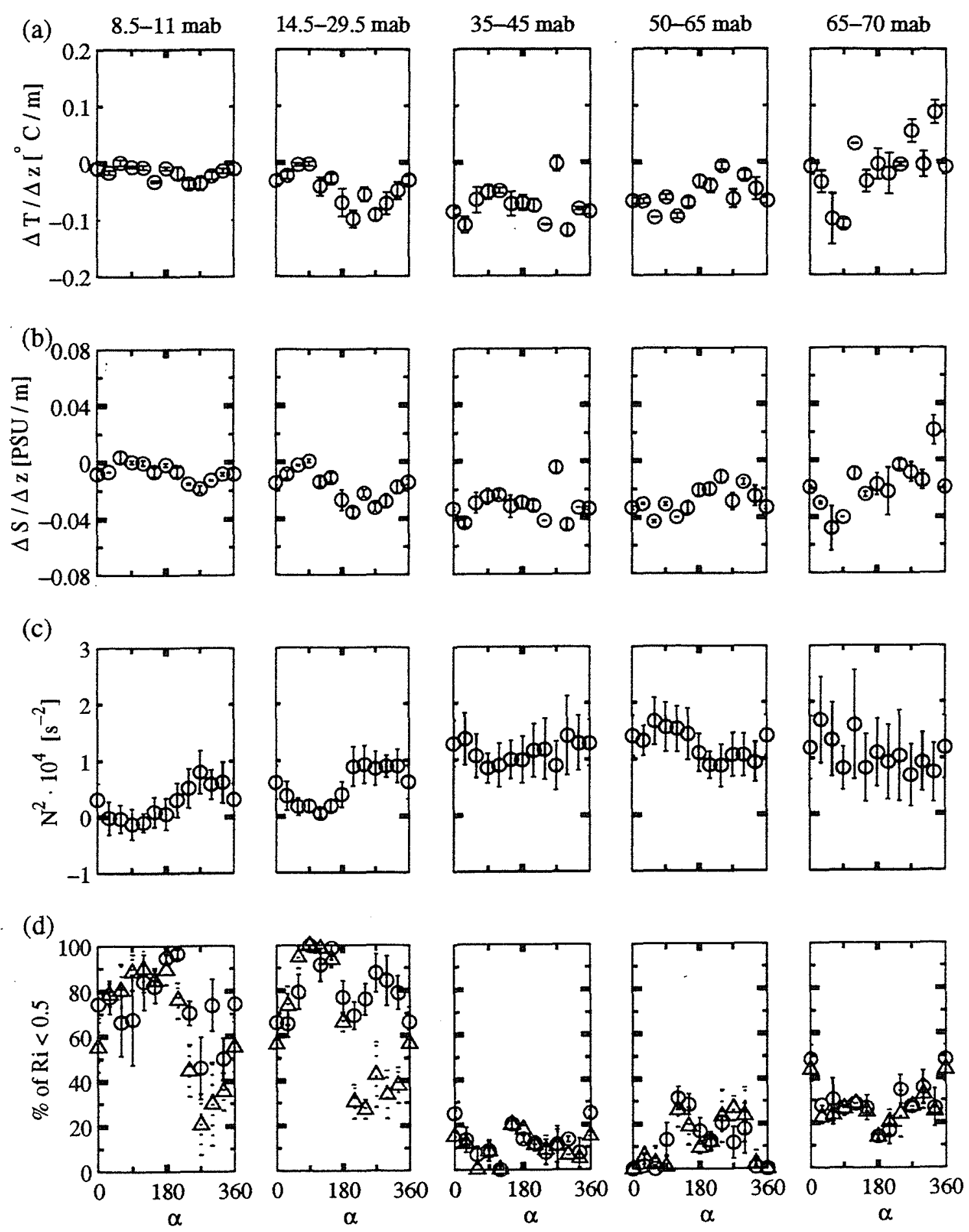

Fig.3.19: Same as Fig.3.15, but for May 14-22. 
(a)

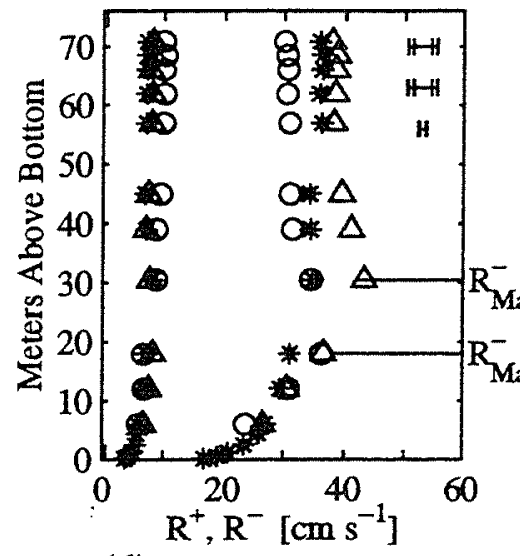

(d)



(b)

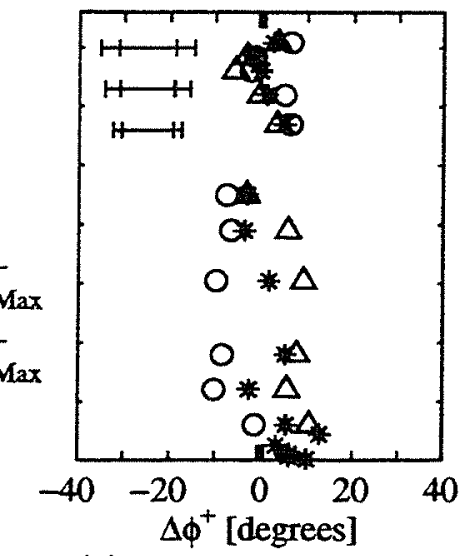

(e)



(c)

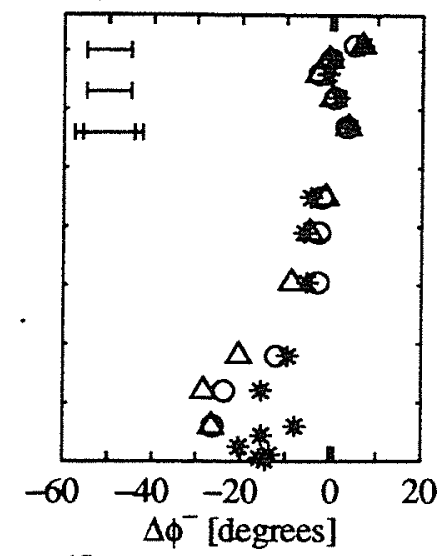

(f)

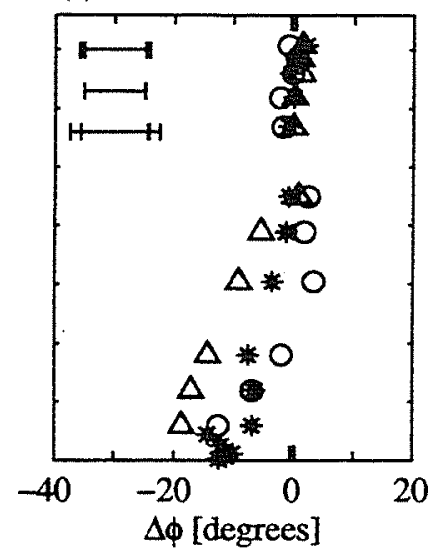

Fig.3.20: Profiles of the $M_{2}$ current ellipse parameters for (asterisks) February 11-March 11, (circles) May 6-14, and (diamonds) May 14-22: (a) magnitudes $R^{+}$(smaller values) and $R^{-}$(larger values), veering angles (b) $\Delta \phi^{+}$and (c) $\Delta \phi^{-}$, (d) amplitudes $\dot{U}_{M a j}(>0)$ and $U_{M \text { in }}(<0)$, (e) rotation of the major axis $\Delta \theta$ (positive counterclockwise), and (f) phase lead $\Delta \phi$. Error limits in the upper left/right hand corner correspond to the (inner error bars) depth-averaged error plus/minus (outer error bars) two standard deviations from the depth-averaged error for (from top downward) May 6-14, May 14-22, and February 11-March 11. Individual error margins were derived from the standard error of the tidal fit at the $95 \%$ confidence level, or, for $\Delta \phi^{ \pm}$and $\Delta \theta$, the compass uncertainty (depending on which is larger). 
(a)



(b)



(c)



Fig.3.21: $N^{2}$ distribution as specified in the numerical model for (a) May 6-14, (b) May 14-22, and (c) July 18-26 and August 6-14. Dashed lines in (a) and (b) are tidal variations representative of the reversal from (plusses) flood to ebb and (diamonds) ebb to flood, and solid lines correspond to the time-mean. Solid and dashed lines in (c) are the time-mean $N^{2}$ distributions for July 18-26 and August 6-14, respectively. 
(a)



(d)

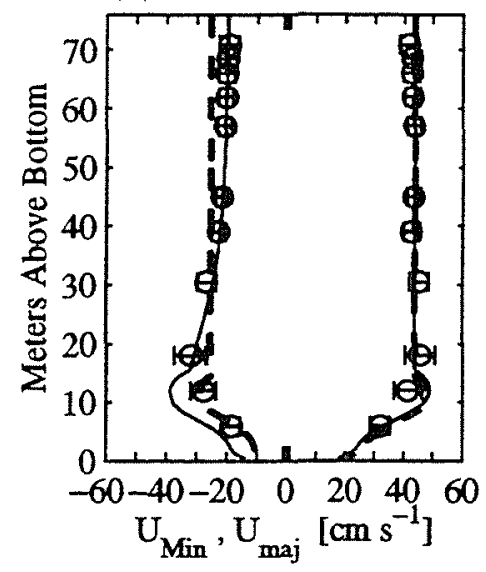

(b)



(e)

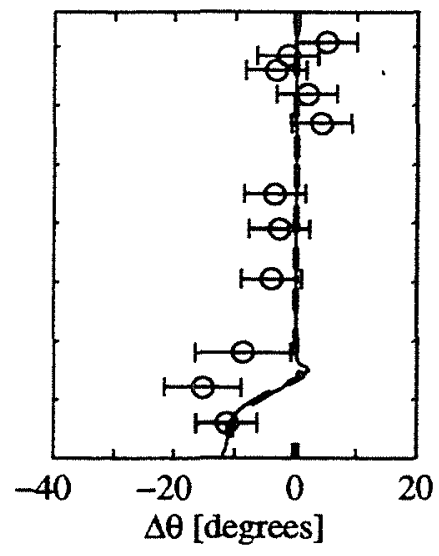

(c)

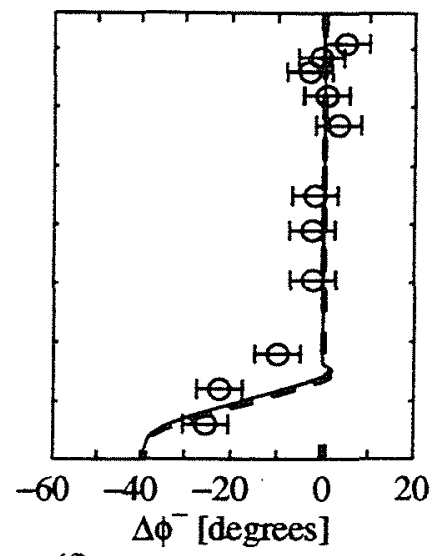

(f)



Fig.3.22: Results for the $M_{2}$ current ellipse from the MY2.5 model for May 6-14 with (dashed) barotropic and (solid) barotropic superimposed by mode-one baroclinic forcing for the tidally varying $N^{2}$ distribution shown in Fig.3.21a. Also shown are (circles) data and (error bars) data uncertainties. Error margins give the standard error of the tidal fit at the $95 \%$ confidence level, or, for $\Delta \phi^{ \pm}$and $\Delta \theta$, the compass uncertainty (depending on which is larger). 
(a)

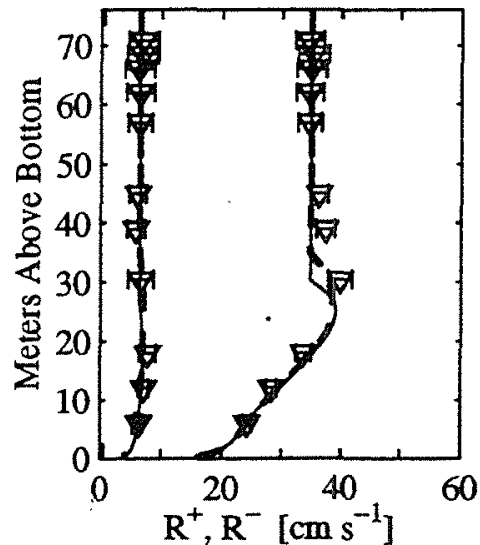

(d)

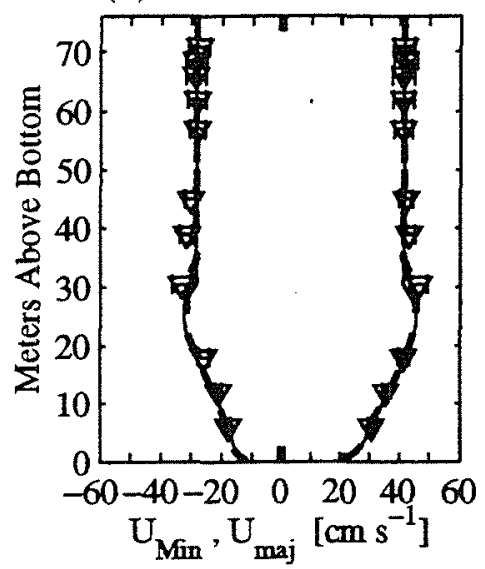

(b)



(e)



(c)



(f)



Fig.3.23: Results for the $M_{2}$ current ellipse from the MY2.5 model for May 14-22 with barotropic forcing for the $N^{2}$ distribution shown in Fig.3.21b. Solid and dashed lines are results for the time-mean and tidally-varying $N^{2}$ distribution, respectively. Also shown are (diamonds) data and (error bars) data uncertainties as in Fig.3.22. 
(a)


(b)
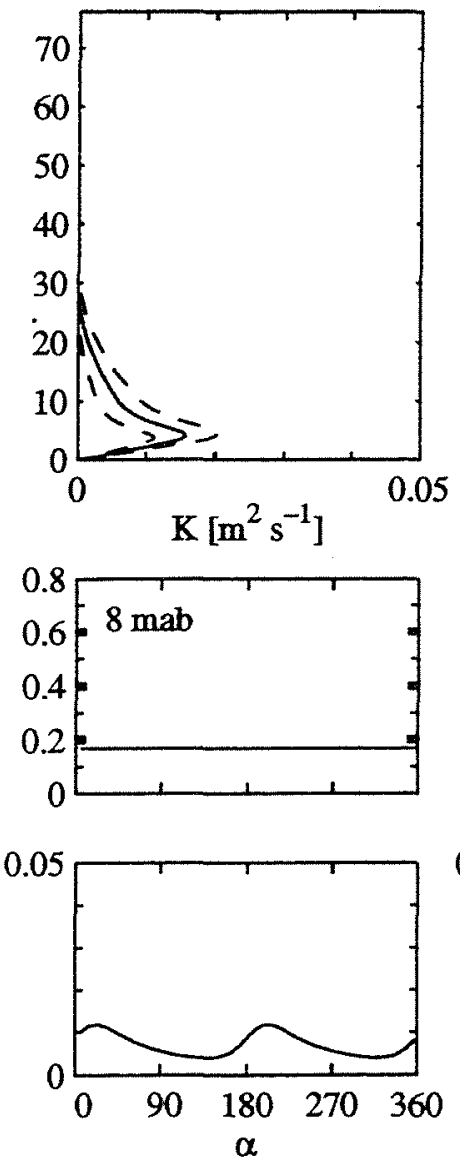

(c)


Fig.3.24: (Top) eddy viscosity profiles from the MY2.5 model representative of the (solid) tidal average and (dashed) variations during a tidal cycle. Also shown are (middle) buoyancy frequencies $N^{2}$ and (bottom) eddy viscosities $K$ at 8 mab versus tidal flow direction $\alpha$ (Fig.3.13). Results are for (a) May 6-14 with barotropic and baroclinic forcing and $N^{2}$ as in Fig.3.21a, (b) May14-22 with time-mean $N^{2}$ as in Fig.3.21b, and (c) May14-22 with tidally varying $N^{2}$ as in Fig.3.21b. Note that the vertical scales of the middle and bottom panels in (a) are different compared to (b) and (c). 
(a)

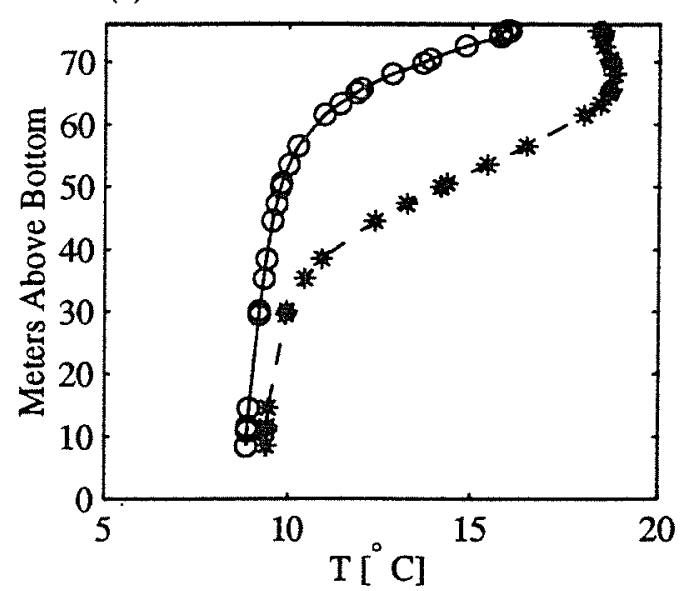

(c)



(b)

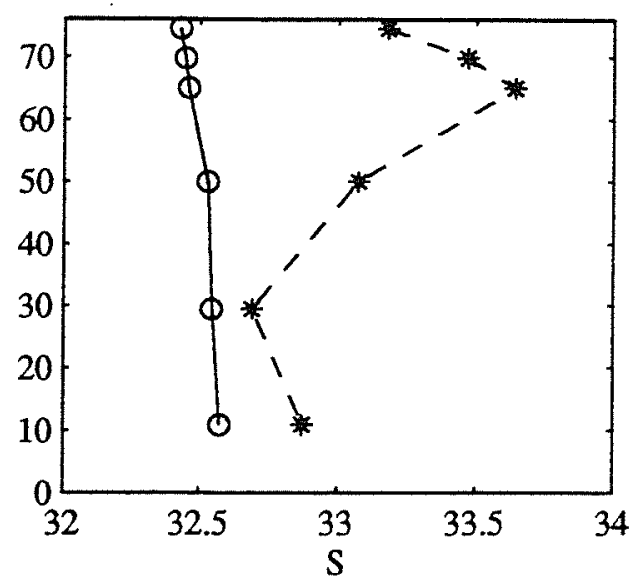

(d)



Fig.3.25: Profiles of time-mean (a) temperature T, (b) salinity S, (c) potential density $\sigma_{\theta}$, and (d) buoyancy frequency squared $N^{2}$ for (solid) July 18-26 and (dashed) August 6-14. 
(a)



(d)

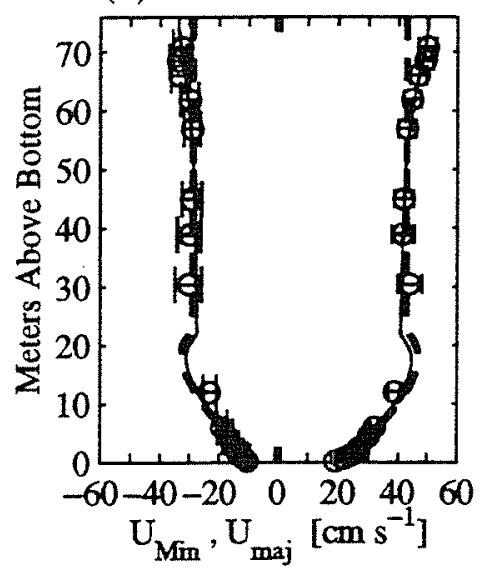

(b)



(e)

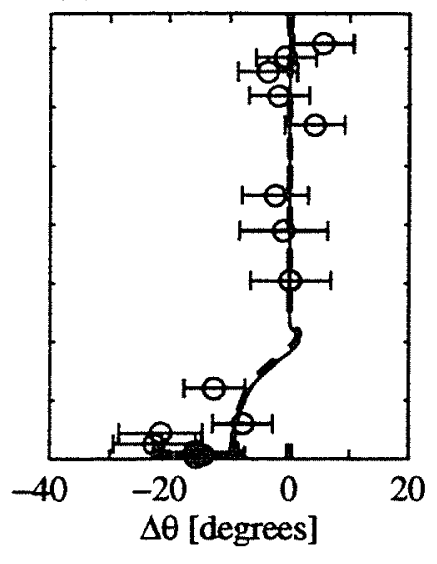

(c)



(f)



Fig.3.26: Profiles of the $M_{2}$ current ellipse parameters for (circles) July 18-26 as in Fig.3.20. Error bars are data uncertainties as desribed in Fig.3.22. Also shown are the MY2.5 model results for the $N^{2}$ distribution shown in Fig.3.21c (solid) with and (dashed) without the superimposed mode-one baroclinic forcing depicted in Fig.3.28. 
(a)

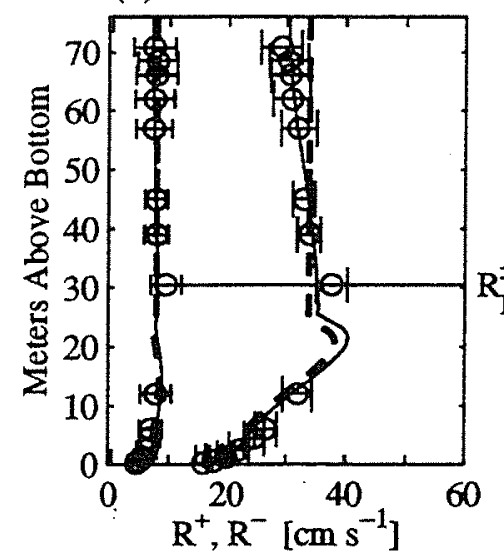

(d)

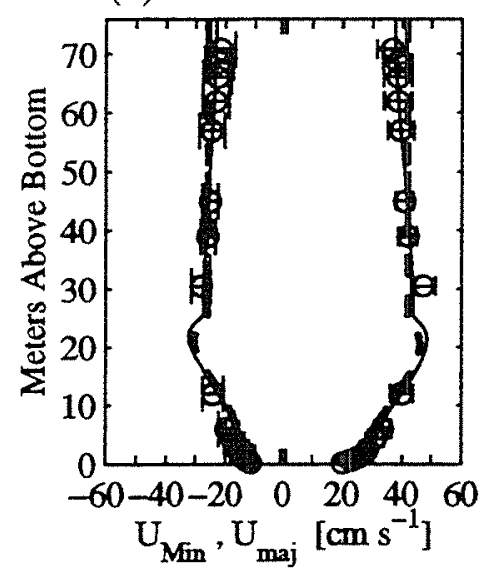

(b)



(e)

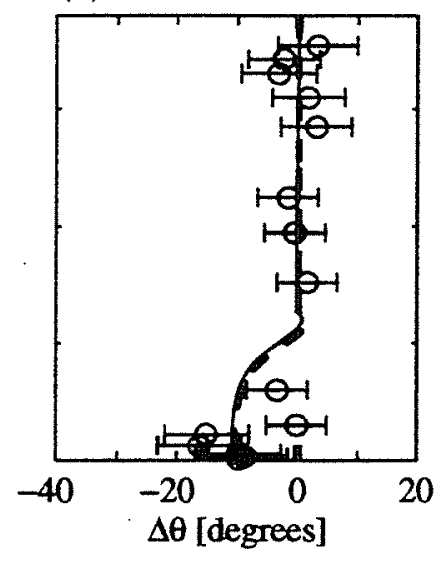

(c)

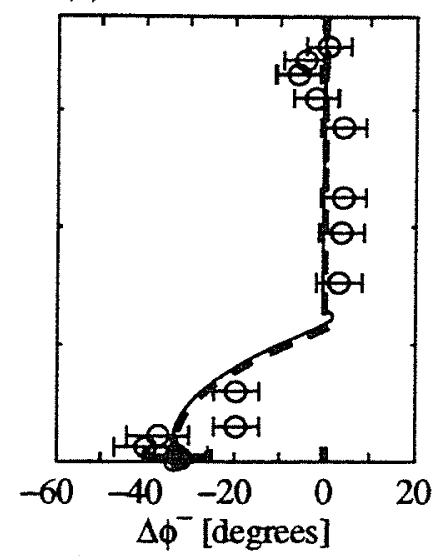

(f)

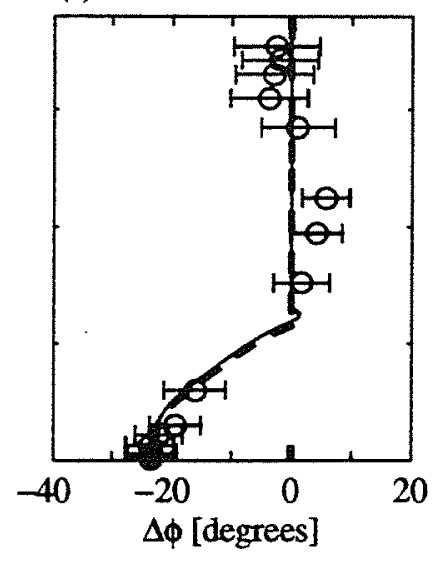

Fig.3.27: Profiles of the $M_{2}$ current ellipse parameters for (circles) August 6-14 similar to Fig.3.20. Error Bars are data uncertainties as desribed in Fig.3.22. Also shown are MY2.5 model results for the $N^{2}$ distribution shown in Fig.3.21c (solid) with and (dashed) without the super- . imposed mode-one baroclinic forcing depicted Fig.3.28. 


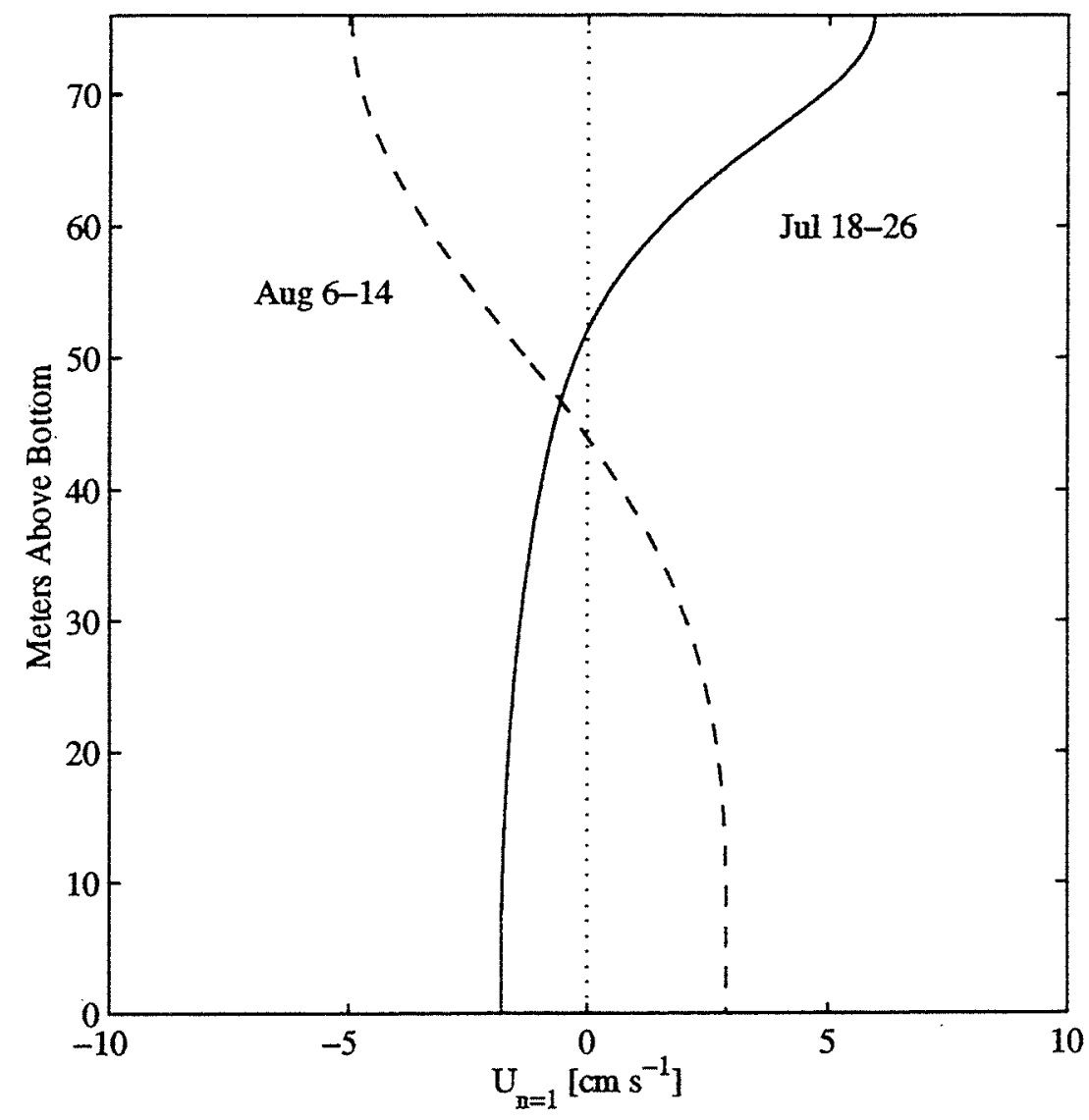

Fig.3.28: Mode-one cross-bank velocity amplitude $U_{n=1}$ for the $N^{2}$ distributions shown in Fig.3.21c. 


\section{Chapter 4}

\section{The Surface Boundary Layer on the Southern Flank of Georges Bank}

\subsection{Introduction}

The subtidal (temporal scales $>33 \mathrm{hrs}$ ) circulation on the southern flank of Georges Bank is described by a clockwise mean circulation whose strength and vertical structure are determined by various processes. Among the most prominent ones are upstream forcing by buoyancy sources such as the Labrador Current and the St. Lawrence River (Chapman and Beardsley, 1989), tidal rectification over the sloping bottom (e.g., Loder, 1980; Butman et al., 1983, Chen, 1992), and, especially in winter, wind forcing. Although rough estimates have been made that relate the depth-averaged flow to the individual forcing mechanisms (Butman and Beardsley, 1987), the relative magnitude of the terms constituting the subtidal momentum balance is largely unknown.

Previous observations have shown the flow on the southern flank of Georges Bank is strongly affected by changes in the wind field (Flagg, 1987, Butman and Beardsley, 1987). The flow response to wind forcing has been investigated using measurements and numerical models (Noble et al., 1985; Greenberg et al., 1997; Brown, submitted). Numerical and observational results predict that the along-bank flow responds strongly to variation in along-bank wind stress, while the response to cross-bank wind forcing is weak. This behavior is explained by the fact that the along-bank winds are roughly aligned with the along-shelf direction (Fig.2.1), hence causing coastal up/downwelling in the Gulf of Maine and adjacent region. The 
resulting sea surface elevations are such that the barotropic response on the southern flank of Georges Bank follows the local isobaths in direction of the wind stress. (e.g., Greenberg et al., 1997; Brown, submitted). Numerical results show that for a crossbank wind stress, up/downwelling occurs only in a few areas where the cross-shelf stress is approximately parallel to the local isobaths (Greenberg et al., 1997). As a consequence, the pressure field consists of significantly smaller gradients than in the case of an along-bank stress, and the regional response is mostly described by Ekman transport.

It follows from the short summary given above that previous investigation of the wind-driven circulation in the greater Gulf of Maine/Georges Bank area has focused primarily on the barotropic response to wind forcing. More specifically, the part of the wind-induced flow that is in geostrophic balance with the pressure field set up by an along-bank wind stress has been examined. In this study, observational evidence consistent with the results described above is limited to a brief description. Emphasis is put on the ageostrophic component of the wind-driven circulation, i.e., the component that is not in balance with the local pressure gradient. In the following, the terms "wind-induced" and "wind-driven" are used to refer to the ageostrophic part of the flow response to wind forcing, although they apply strictly speaking to both the geostrophic and the ageostrophic currents.

In this chapter, rough estimates are made of the individual terms in the depthaveraged momentum balance for times representative of winter and summer, and the estimated sea surface slopes are compared to predictions from a three-dimensional numerical circulation model. The analysis of the wind-induced (ageostrophic) flow is performed using Price et al.'s (1987) "coherent ensemble average method", which has been applied with great success to investigate the surface layer in the deep ocean (e.g., Schudlich and Price, 1998; Chereskin, 1995). Differences from Price et al.'s (1987) approach arise from complications posed by the shallow water depth, nearness of coastal boundaries, and lateral density gradients on Georges Bank, which require additional steps be taken to separate the wind-driven currents from the background flow field. The vertical distribution of the wind-induced currents is investigated for the long-term mean representative of winter and summer, and for individual wind events at times when such analysis was possible. Observations are brought into context with previous results from deep ocean studies and predictions from simple analytical models. 


\subsection{Subtidal Dynamics}

\subsubsection{SF Surface Mooring}

Analysis of the subtidal circulation requires the knowledge of density gradients in both the cross- and along-bank direction. For this reason, hourly averaged data from the southern flank mooring (SF) located at $40^{\circ} 58.0^{\prime} \mathrm{N}, 67^{\circ} 19.2^{\prime} \mathrm{W}$ in 76 -m water depth were incorporated in this study, in addition to the measurements taken at the ST1 and ST2 moorings described in Chapters 2 and 3 (Fig.4.1). The deployment periods of the SF surface mooring were October 27, 1994-March 16, 1995 (deployment I), and April 26-August 23, 1995 (deployment II). The buoy broke loose during deployment I, so that the available data record ends on March 4. Measurements taken by the SF mooring include air temperature, water temperature, conductivity, photosynthetically active radiation, light transmission, and fluorescence at various depth. Here, only sea surface temperature measurements at 1 -m depth (75 mab), and temperature and conductivity measurements taken in 5-m increments between 5- and 50-m depth (26 and $71 \mathrm{mab}$ ) are used. Measurements were taken by Seabird temperature and conductivity sensors, with uncertainties comparable to those of the SeaCATs listed in Chapter 2. The sampling rate of the instruments was $1 \mathrm{~min}$, and data were averaged in hourly bins for storage. No data were returned by the temperature and conductivity sensors at $40-\mathrm{m}$ depth (36 mab) for deployment II, and the conductivity sensor at $20-\mathrm{m}$ depth $(56 \mathrm{mab}$ ) for deployment I. The conductivity and temperature records at 5 -m depth $(71 \mathrm{mab})$ were short during deployment I and ended on Feb 10.

\subsubsection{Subtidal Momentum Equations}

The depth-averaged momentum equations for the subtidal flow are

$$
\begin{gathered}
\frac{1}{D} \int_{-D}^{0}\left(<\frac{\partial u}{\partial t}>+<u \frac{\partial u}{\partial x}>+<v \frac{\partial u}{\partial y}>+<w \frac{\partial u}{\partial z}>-f<v>\right) d z= \\
-g<\frac{\partial \eta}{\partial x}>-\frac{g}{\rho_{0}} \frac{1}{D} \int_{-D}^{0}<\frac{\partial \rho}{\partial x}>(D+z) d z+\frac{<\tau_{W}^{x}>}{\rho_{0} D}-\frac{<\tau_{b}^{x}>}{\rho_{0} D}
\end{gathered}
$$




$$
\begin{gathered}
\frac{1}{D} \int_{-D}^{0}\left(<\frac{\partial v}{\partial t}>+<u \frac{\partial v}{\partial x}>+<v \frac{\partial v}{\partial y}>+<w \frac{\partial v}{\partial z}>+f<u>\right) d z= \\
-g<\frac{\partial \eta}{\partial y}>-\frac{g}{\rho_{0}} \frac{1}{D} \int_{-D}^{0}<\frac{\partial \rho}{\partial y}>(D+z) d z+\frac{<\tau_{W}^{y}>}{\rho_{0} D}-\frac{<\tau_{b}^{y}>}{\rho_{0} D}
\end{gathered}
$$

where angular brackets denote a low-pass filter operator with half power period $33 \mathrm{hrs}, z$ is positive upward ( $z=0$ at the surface), $\eta$ is sea surface elevation, $D=$ $76 \mathrm{~m}$ is water depth at ST1, and $\rho_{0}$ is a reference density. Based on measurements at the ST1, ST2, and SF moorings, all terms in (1) can be estimated, except those involving the sea surface slopes $\frac{\partial \eta}{\partial x}$ or $\frac{\partial \eta}{\partial y}$, and the velocity gradients in the $y$ and $z$-direction. Nonlinear expressions that contain vertical current shear may be removed by writing the momentum balance in flux form, i.e., by adding to the left hand side of (1) the continuity equation multiplied by $u$ and $v$

$$
(u, v)\left(<\frac{\partial u}{\partial x}>+<\frac{\partial v}{\partial y}>+<\frac{\partial w}{\partial z}>\right)=0
$$

Taking this step allows the advective terms to be rewritten as

$$
\begin{aligned}
\left(<u \frac{\partial u}{\partial x}>+<v \frac{\partial u}{\partial y}>+<w \frac{\partial u}{\partial z}>\right)+ & \left(<u \frac{\partial u}{\partial x}>+<u \frac{\partial v}{\partial y}>+<u \frac{\partial w}{\partial z}>\right) \\
= & <\frac{\partial\left(u^{2}\right)}{\partial x}>+<\frac{\partial(u v)}{\partial y}>+<\frac{\partial(u w)}{\partial z}> \\
\left(<u \frac{\partial v}{\partial x}>+<v \frac{\partial v}{\partial y}>+<w \frac{\partial v}{\partial z}>\right)+ & \left(<v \frac{\partial u}{\partial x}>+<v \frac{\partial v}{\partial y}>+<v \frac{\partial w}{\partial z}>\right) \\
& =<\frac{\partial(u v)}{\partial x}>+<\frac{\partial\left(v^{2}\right)}{\partial y}>+<\frac{\partial(v w)}{\partial z}>.
\end{aligned}
$$

With the boundary conditions $w(z=0)=w(z=-D)=0$, the depth-average of (3) is given by 


$$
\begin{aligned}
& \frac{1}{D} \int_{-D}^{0}\left(<\frac{\partial\left(u^{2}\right)}{\partial x}\right\rangle+<\frac{\partial(u v)}{\partial y}>\left.+<\frac{\partial(u w)}{\partial z}>\right) d z= \\
& \frac{1}{D} \int_{-D}^{0}\left(<\frac{\partial u^{2}}{\partial x}>+<\frac{\partial u v}{\partial y}>\right) d z \\
& \frac{1}{D} \int_{-D}^{0}\left(<\frac{\partial(u v)}{\partial x}>+<\frac{\partial\left(v^{2}\right)}{\partial y}>+\right. \\
& \frac{1}{D} \int_{-D}^{0}\left(<\frac{\partial(v w)}{\partial z}>\right) d z=
\end{aligned}
$$

The nonlinear terms on the right hand side of (4) scale according to

$$
\begin{aligned}
& <\frac{\partial\left(u^{2}\right)}{\partial x}>+<\frac{\partial(u v)}{\partial y}>\sim \frac{U^{2}}{L_{X}}+\frac{U V}{L_{Y}} \\
& <\frac{\partial(u v)}{\partial x}>+<\frac{\partial\left(v^{2}\right)}{\partial y}>\sim \frac{U V}{L_{X}}+\frac{V^{2}}{L_{Y}},
\end{aligned}
$$

where $L_{Y}$ and $L_{X}$ are along- and cross-bank length scales, respectively, and $U$ and $V$ are scale velocities. Compared to the depth-averaged subtidal flow, the tidal velocity amplitudes are large in both the $x$-and $y$-direction, so that to a first approximation $U \sim V$. On the basis that the bottom topography is largely defined by the cross-bank bottom slope, it is assumed that $L_{Y}>L_{X}$, and the $y$-derivatives in (5) can be ignored with respect to the derivatives in $x$. Substituting the lowest order terms of (4) in (1) and defining

$$
[]=\frac{1}{D} \int_{-D}^{0} d z
$$

the depth-averaged momentum balance reduces to

$$
\begin{aligned}
& \frac{\partial[u]}{\partial t}+\left[\frac{\partial\left(u^{2}\right)}{\partial x}\right]-f[v]=-g \frac{\partial \eta}{\partial x}-\frac{g}{\rho_{0}}\left[\frac{\partial \rho}{\partial x}(D+z)\right]+\frac{\tau_{W}^{x}}{\rho_{0} D}-\frac{\tau_{b}^{x}}{\rho_{0} D} \\
& \frac{\partial[v]}{\partial t}+\left[\frac{\partial(u v)}{\partial x}\right]+f[u]=-g \frac{\partial \eta}{\partial y}-\frac{g}{\rho_{0}}\left[\frac{\partial \rho}{\partial y}(D+z)\right]+\frac{\tau_{W}^{y}}{\rho_{0} D}-\frac{\tau_{b}^{y}}{\rho_{0} D}
\end{aligned}
$$


where angular brackets denoting the low-pass operator have been omitted.

\subsubsection{Estimation of Subtidal Momentum Terms}

The first and third terms on the left hand side of (7) were estimated from depth-averaged current data taken at ST1. Wind stress was inferred from ST1meteorological data using the Large and Pond (1981) neutral stability algorithm. Computations of bottom stress were based on the quadratic drag law

$$
\left(\tau_{b}^{x}, \tau_{b}^{y}\right)=c_{D} \cdot U_{t o t}\left(u_{t o t}, v_{t o t}\right)
$$

applied at $6 \mathrm{mab}$, where $c_{D}=1.75 \cdot 10^{-3}$ is a representative drag coefficient (Table 3.1). In (8), the subscript tot refers to the total measured velocities, i.e., the tidal and subtidal currents combined. After application of (8), results were low-pass-filtered to remove tidal variation of bottom stress.

Density gradients were approximated by

$$
\begin{aligned}
& \frac{\partial \rho}{\partial x}=\frac{\rho_{S T 2}-\rho_{S T 1}}{\Delta X} \\
& \frac{\partial \rho}{\partial y}=-\frac{\rho_{S T 1}-\rho_{S F}}{\Delta Y}
\end{aligned}
$$

where $\Delta X=12 \mathrm{~km}$ and $\Delta Y=23 \mathrm{~km}$ are the horizontal distances from ST2 to ST1 and SF to ST1, respectively. Temperature, salinity, and density at ST2 and SF were interpolated linearly with depth to obtain gradient estimates at the instrumentation depths of the ST1 mooring. The computed density gradients were used to estimate the baroclinic pressure forcing on the right hand side of (7). It is expected that expression (9) yields no more than very rough approximations of the lateral density variations at ST1. This is partly because the separation distances $\Delta X$ and $\Delta Y$ at times exceed the baroclinic Rossby radius $R \approx 7-24 \mathrm{~km}$ representative of spring and summer. Additional error arises from the fact that the estimated gradients are not centered at ST1, but at midpoints between adjacent moorings.

The advective terms on the left hand side of (7) were approximated by 


$$
\begin{aligned}
& {\left[\frac{\partial\left(u^{2}\right)}{\partial x}\right]=\left(\frac{u_{S T 2}^{2}-u_{S T 1}^{2}}{\Delta X}\right)} \\
& {\left[\frac{\partial(u v)}{\partial x}\right]=\left(\frac{(u v)_{S T 2}-(u v)_{S T 1}}{\Delta X}\right)}
\end{aligned}
$$

where measurements at ST1 were interpolated to the current meter depths at ST2. Similar to the density gradients, cross-bank advection was evaluated at mid-distance between ST1 and ST2. Because of the sparse vertical resolution of the current measurements at ST2, estimates of cross-bank advection are particularly crude and should be interpreted with great caution. This is especially true after May 17 when one of the three current meters at ST2 failed. Since no measurements were taken that would allow for the direct estimation of the barotropic pressure forcing, rough estimates were obtained by solving expression (7) for the terms containing the sea surface slope.

\subsubsection{Subtidal Momentum Balance}

The individual terms in the subtidal momentum balance are investigated for two periods representative of weakly stratified conditions with high winds and strongly stratified conditions with low winds (Figs.4.2 and 4.3). Similar to observations from previous years described by Butman and Beardsley (1987), wind stress was larger in winter and early spring than in late spring and summer. Exceptions occurred in $\mathrm{Au}$ gust 1995, when intermittent events of large southwesterly winds marked the passage of tropical storms. Wind stress variance was about 5 times greater from February to mid-May than from mid-May to August, in good agreement with Brown's (submitted) results from 1986/87 data indicating surface wind stress contained 4 times more energy in winter than in summer. The cross-bank component of the wind stress was predominantly off-bank during the winter months, indicative of strong seaward winds during the cold season. During the summer months, the preferred wind orientation was along-bank and varied between northeastward and southwestward winds.

The first investigation period (period I) extends from February 1-May 5, when measurements indicate cross- and along-bank variations of temperature, salinity, and density were small (Fig.4.4). Density gradients were mostly positive on-bank 
during period I due to the influence of slightly fresher water over the outer bank (Fig.4.4a, bottom). During May 6-28, a Slope Water intrusion (SWI) advected warm, saline Slope Water past ST1, resulting in large horizontal and vertical stratification (Figs.4.3 and 4.4, see also Chapter 3). This event is excluded from the analysis of the subtidal momentum balance, since it is not characteristic of typical spring or summer conditions. The second investigation period (period II) covers May 29-August 4 when seasonal intensification of the Shelf-Slope front caused significant lateral variation of the temperature and salinity fields. During period II, temperature displays strong off-bank gradients in the surface layer, and smaller onbank gradients at depth (Fig.4.4a, top). The gradient reversal occurred 15-20 m below the surface and indicates the formation of a seasonal thermocline. Surface heating and tidal mixing produced a homogeneous water mass on the shallow bank plateau that was warmer than the sub-thermocline water further south, but colder than the heated layer above the temperature interface. Salinity shows different behavior, with gradients that varied around zero near the surface and were positive off-bank at depth indicative of the base of the Shelf-Slope front (Fig.4.4a, middle). Above the thermocline, density gradients were largely determined by the temperature field and positive on-bank (Fig.4.4a, bottom). Below the thermocline, salinity and temperature combined to produce increasing densities toward the outer bank. With exception of the May SWI and a similar event in August almost immediately following period II, temperature, salinity, and density display significantly weaker variation in the along-bank than in the cross-bank direction (Fig.4.4b). The August SWI cannot be observed in cross-bank gradients, since the ST2 data record ends before the event.

Table (4.1) summarizes the temporal means and standard deviations of all terms in (7). Since no data were taken from February 1-3 at ST2 and March 5-April 26 at $\mathrm{SF}$, the advective terms and baroclinic pressure gradients were averaged over times surrounding these data gaps in the cross- and along-bank momentum balance, respectively. For the purpose of estimating expressions containing the sea surface slope, the unknown terms were set to zero when data at ST1 or SF were not available.

The listed values suggest that during both periods I and II, the contribution of the sea surface gradients was significant in both the along- and cross-bank momentum balance. During period I, the increase of density in the on-bank direction produced a depth-averaged buoyancy-driven pressure gradient that was positive on- 
bank, thus adding to the estimated barotropic pressure forcing. For period II, the density gradient reversal below the pycnocline resulted in depth-averaged baroclinic pressure forcing that opposed the barotropic forcing caused by the sea surface slope. The time-mean, depth-averaged along-bank flow was southwestward and stronger in summer than in winter and early spring, in agreement with earlier observations (e.g., Butman and Beardsley, 1987).

Temporal variation of the along-bank flow was larger than the time-mean value in period I and about half the time-mean in period II. During both investigation periods, the along-bank flow and wind stress were highly correlated, in agreement with earlier results by Noble et al. (1985). For period I, the peak correlation between $[v]$ and $\tau_{W}^{y}$ is 0.65 , with the current lagging the wind stress by 9 hrs. The computed correlation coefficient is significant at the $95 \%$ confidence level $\left(c_{95}=0.25\right)$ obtained from cross-correlation time scales. In their analysis of long-term current and wind measurements, Noble et al. (1985) found the along-bank currents lag the alongbank wind by 9 -hrs on the southern flank of Georges Bank, in agreement with the observations presented here. The observed phase-shift represents the time scale of the regional response to wind forcing, i.e., the time elapsed before the windinduced pressure field has fully developed. During period II, the peak correlation between the along-bank currents and wind stress is 0.52 at the $95 \%$ confidence level $\left(c_{95}=0.30\right)$, with the current lagging the wind by $3 \mathrm{hrs}$. The significantly shorter phase-lag between the wind and currents during the more stratified season supports earlier results by Brown et al. (1985), who found the bottom pressure gradient lagged the along-shelf wind off Nantucket Shoals by about 12-21 hrs in winter as opposed to $5-13.5$ hrs in summer.

The variation of the along-bank wind stress is also related to changes in the depth-averaged cross-bank flow. Correlation coefficients between $\tau_{W}^{y}$ and $[u]$ are 0.53 and 0.35 for periods I and II, respectively, with $95 \%$ confidence levels that are similar to those given above $\left(c_{95}=0.25\right.$ and $c_{95}=0.30$ for periods I and II, respectively). Characteristic phase-shifts of the peak correlation are $0-2 \mathrm{hrs}$ with the current lagging the wind. The quick response of the cross-bank current to alongbank wind forcing may indicate the observed correlation describes Ekman transport to the right of the wind. A typical spin-up time of the Ekman transport is about $\frac{1}{f} \approx 3 \mathrm{hrs}$ and roughly consistent with the computed phase-lag. In summer when winds are weak and the water column becomes increasingly stratified, the Ekman 
response is limited to a thin surface layer, while other processes determine the flow field at greater depth. Thus, the correlation between the depth-averaged cross-bank flow and along-bank wind stress is stronger in winter than in summer.

\subsubsection{Comparison to Numerical Circulation Models}

To check whether the indirect estimates of barotropic pressure forcing listed in Table 4.1 are meaningful, results were compared to predictions from a threedimensional numerical circulation model. The model is prognostic and nonlinear, has a free surface, and uses a finite element mesh that covers the Gulf of Maine, Georges Bank, Scotian Shelf, and adjacent region. Model forcing are the barotropic $M_{2}$ tide and surface wind stress. Initial conditions and wind forcing are from bimonthly averages of climatological temperature, salinity, and wind data. With the exception of cross-bank density gradients representative of winter, model winds and density fields are in good agreement with the observations (see below for closer investigation of the winter case). Details about the numerical scheme, boundary conditions, and model equations can be found in Naimie (1996), and Lynch et al. (1996). All values cited here are from Naimie (personal communication).

For the periods February through April and June through July, the model predicts mean cross-bank gradients $g \frac{\partial \eta}{\partial x}=2.8 \cdot 10^{-6} \mathrm{~m} \mathrm{~s}^{-2}$ and $11.0 \cdot 10^{-6} \mathrm{~m} \mathrm{~s}^{-2}$, respectively. These values are of the same order and sign as the mean gradients listed in Table 4.1, indicating the derived estimates are reasonable. The corresponding Coriolis terms $f[v]$ are $4.3 \mathrm{~m} \mathrm{~s}^{-2}\left([v]=4.6 \mathrm{~cm} \mathrm{~s}^{-1}\right)$ from February-April and $9.4 \mathrm{~m} \mathrm{~s}^{-2} \quad\left([v]=9.9 \mathrm{~cm} \mathrm{~s}^{-1}\right)$ from June-July, also in support of the observations. Closer investigation shows the discrepancy of about $1.7 \cdot 10^{-6} \mathrm{~m} \mathrm{~s}^{-2}$ between the predicted and estimated $g \frac{\partial \eta}{\partial x}$ in winter (February-April) can be largely explained by the model climatology: opposed to the observed on-bank density gradient, the numerical model assumes density increases in the off-bank direction. The result is a depth-averaged baroclinic pressure gradient in the cross-bank direction of $-0.4 \cdot 10^{-6} \mathrm{~m} \mathrm{~s}^{-2}$ compared to $1.1 \cdot 10^{-6} \mathrm{~m} \mathrm{~s}^{-2}$ inferred from the measurements.

In the along-bank direction, model predictions are $g \frac{\partial \eta}{\partial y}=-1.1 \cdot 10^{-6} \mathrm{~m} \mathrm{~s}^{-2}$ from February to April and $g \frac{\partial \eta}{\partial y}=-3.3 \cdot 10^{-6} \mathrm{~m} \mathrm{~s}^{-2}$ from June to July, thus opposing the sign of the estimates in Table 4.1. The predicted cross-bank velocities are about $0.5 \mathrm{~cm} \mathrm{~s}^{-1}\left(f[u]=0.5 \cdot 10^{-6} \mathrm{~m} \mathrm{~s}^{-2}\right)$ and off-bank (on-bank) during the winter (summer) months, as opposed to $[u] \approx-2 \mathrm{~cm} \mathrm{~s}^{-1}\left(f[u] \approx-(1.8-1.9) \cdot 10^{-6} \mathrm{~m} \mathrm{~s}^{-2}\right)$ 
off-bank flow based on measurements. The discrepancy between the numerical model and observational results may have several reasons: the most obvious explanation is that the estimated cross-bank transport and hence the inferred sea surface slope are subject to the measurement uncertainty of the VMCMs. However, time-mean offbank flow of about $2 \mathrm{~cm} \mathrm{~s}^{-1}$ is observed at all VMCMs, implying the unlikely case that all current meters were biased simultaneously toward negative values. Another possibility is that the along-bank pressure gradient and geostrophic cross-bank flow are sensitive to local variations in bottom topography, which are either not included in the model bathymetry or not fully resolved by the numerical grid. Last, the along-bank momentum balance predicted by the model may not be described by a near-balance between the pressure and Coriolis terms, but require the consideration of the bottom friction and/or nonlinear advection terms to lowest order. Model solutions may support results by Beardsley and Winant (1979) and Chapman et al. (1986), who suggest upstream forcing creates an along-shelf pressure gradient on the southern flank of Georges Bank and in the Mid-Atlantic Bight which is positive northeastward (i.e., $-y$ in the reference system chosen here). In this scenario, buoyant fresh water, e.g. due to river runoff and coastal currents originating further north, follows lines of constant bottom topography and sets up an along-shelf pressure gradient which balances bottom friction (Beardsley and Hart, 1978). Further investigation of the mechanisms determining the along-bank momentum balance is reserved for future studies.

Results from a linear homogeneous three-dimensional circulation model predict the along-shelf currents lag the wind with increasing distance seaward, in good agreement with observation (Greenberg et al., 1997). Using Lynch et al.'s (1992) finite-element model with spatially varying eddy viscosity and linear bottom friction, Greenberg et al. (1997) solved for the pressure field caused by a wind stress of given magnitude and direction. For an along-bank wind stress corresponding to the observed standard deviation during period I, predictions at ST1 are $\left|g \frac{\partial \eta}{\partial x}\right|=3.5 \cdot 10^{-6} \mathrm{~m} \mathrm{~s}^{-2}$, with the sea surface sloping upward toward the right of the wind. This value corresponds to about half the standard variation of the barotropic pressure gradient listed in Table 4.1. Model results also indicate that cross-bank wind stress sets up a sea surface slope at ST1 which opposes the wind direction, giving $\left|g \frac{\partial \eta}{\partial x}\right|=1.5 \cdot 10^{-6} \mathrm{~m} \mathrm{~s}^{-2}$ for wind magnitudes representative of the observed standard deviation during period I. The combined along- and cross-bank variation of 
the wind stress may thus account for $50 \%$ or more of the variance in the cross-bank sea surface gradient listed in Table 4.1. Model results for either cross- or along-bank wind predict along-bank pressure gradients that are about one order of magnitude smaller than the standard deviations of the along-bank sea surface gradients inferred from data. In the light of Chapman et al.'s (1986) study, this comparison suggests that variation of the along-bank pressure gradient is related to variation in up- or downstream forcing not captured by Lynch et al.'s (1992) numerical model.

\subsubsection{Comparison of Wind and Bottom Stress}

Previous observations have shown surface wind stress is balanced by Ekman transport in the deep ocean (e.g., Price et al., 1987; Chereskin, 1995). In shallow coastal regions such as the inner shelf, the surface transports may be substantially weaker than the deep water Ekman transport. Model results indicate this behavior can be explained by merging of the surface and bottom boundary layers, which results in vertical stress divergence everywhere in the water column (Ekman, 1905; Lentz, 1992). If the vertical scale of the surface boundary layer exceeds the water depth, part of the wind-induced momentum penetrates to the bottom, where it is balanced by bottom friction. In the extreme cases of very shallow water and particularly strong winds, a near-balance between the surface and bottom stress may be achieved (Winant and Beardsley, 1979).

Comparison of wind and bottom stress gives a first indication about whether the wind-induced surface momentum flux penetrates to the bottom at the $76-\mathrm{m}$ deep ST1 mooring site. Time series of low-pass-filtered wind and bottom stress estimates are shown in Fig.4.5, with the bottom stress computed as described in section 4.2.3. Cross-bank components of the wind and bottom stress show little correlation in winter and summer, with correlation coefficients that are insignificant at the $80 \%$ confidence level for both the energetic wind season February to midMay and the calmer period mid-May to August. Even during the largest wind events in winter, the cross-bank bottom stress is about one order of magnitude smaller than the cross-bank wind stress (Fig.4.5, top). These observations indicate turbulent momentum introduced at the surface does not penetrate to the bottom, so that no significant response of the cross-bank bottom stress to wind forcing occurs. Analytical considerations reveal a characteristic depth scale describing the region 
of frictional influence during homogeneous conditions is $\ell_{W}=\frac{\kappa u_{* W}}{f}$, where $u_{*_{W}}=$ $\left(\frac{\tau_{W}}{\rho_{0}}\right)^{1 / 2}$, and $\tau_{W}$ is the wind stress magnitude (Madsen, 1977). Using Madsen's (1977) scaling expression, typical boundary layer thicknesses representative of the strongest wind events are about 80-90 m. Thus, the surface boundary layer depth during winter storms is comparable to the water depth, suggesting the wind-induced momentum flux is to first order balanced by Coriolis acceleration rather than by bottom stress.

In the along-bank direction, bottom stress estimates are also small compared to the wind stress (Fig.4.5, bottom). However, a weak response to wind forcing is observed during some large events, e.g., Feb.2-9, Feb.27-29, Apr.5-6, and the two tropical storms in August. The correlation between $\tau_{b}^{y}$ and $\tau_{W}^{y}$ is significant at the $95 \%$ confidence level ( $c_{95}=0.25$ and $c_{95}=0.31$ for periods I and II, respectively). For period I, the peak correlation is 0.57 with the bottom stress lagging the wind by $11 \mathrm{hrs}$, while for period II, the maximum correlation is 0.47 at about 0 hrs phase lag. These values suggest that the along-bank bottom stress responds strongly to variations in along-bank wind during both periods I and II, but the time scales of the response vary depending on season. Vertical transfer of the wind-induced turbulent momentum to the bottom cannot explain the observed behavior, since statistically significant correlation is limited to only one of the two horizontal stress components, i.e., the along-bank component. On the other hand, the computed phase-shift between the along-bank wind and bottom stress is within 2-3 hrs of the estimated phase lag between the depth-averaged along-bank currents and surface winds (section 4.2.4). The inference is that the wind-induced along-bank currents cause a bottom stress that is aligned with the flow, thus correlating the along-bank wind and bottom stress.

\subsubsection{Surface Mixed Layer Depth}

The thickness of the surface mixed layer depends on physical mechanisms such as wind forcing, surface heating, and other processes sustaining the ambient density field. Time-series of mixed layer depth, here defined as the depth where the observed temperature is within $0.05^{\circ} \mathrm{C}$ from observations $1 \mathrm{~m}$ below the surface, show large variability on time scales of days to months (Fig.4.6a). The mixed layer is deepest in winter when winds are large and surface cooling occurs (Fig.4.6a-c). In spring and 
summer, increasing solar radiation and vertical stratification limit the penetration depth of wind-induced turbulence (Figs.4.6 and 4.3).

For the simplest, one-dimensional case, the vertical extent of the surface mixed layer is defined by the energy balance between shear-generated turbulence immediately underneath the homogeneous surface waters, and entrainment of denser water from below. Assuming no surface heat flux or lateral advection, and taking the initial stratification as vertically constant, Pollard et al. (1973) found

$$
h_{p r t}=A \frac{u_{* W}}{\left(N_{I} f\right)^{1 / 2}}
$$

describes the mixed layer depth, where $N_{I}$ is the buoyancy frequency at the base of the mixed layer, and $A$ is a proportionality constant. Although (11) was derived for strictly one-dimensional conditions without surface heating or cooling, Lentz (1992) found good agreement between the observed and predicted boundary layer thicknesses in coastal upwelling regions using $A=1$. Lentz (1992) argued crossshelf heat transport induced by along-shelf winds balances the surface heat flux, so that (11) gives reasonable results even in the presence of strong solar radiation and lateral advection.

In contrast to the cases studied by Lentz (1992), Georges Bank is not an upwelling region in the typical sense due to its large distance $(\approx 300 \mathrm{~km})$ from the coast (Fig.2.1). Brink (1983) showed the nature of the wind-driven response over a shallow submarine bank depends strongly on the ratio $\frac{D_{0}}{\ell_{W}}$, where $D_{0}$ is the water depth on the crest of the bank, and $\ell_{W}$ is a scale thickness describing the vertical extent of the wind-induced flow. For $D_{0} \geq \ell_{W}$, along-bank wind stress is balanced by Ekman transport that flows across the bank. If $D_{0}<\ell_{W}$, bottom friction rotates and inhibits the wind-driven surface transport in the shallow regions, while the full Ekman transport develops in the deep waters off the bank. In order to preserve the continuity of the cross-bank flow, a compensating transport must develop near the bottom and up/downwelling occurs. On Georges Bank, the crest of the bank has an average depth of $D_{0} \approx 50 \mathrm{~m}$. Taking Madsen's (1977) parameterization of the wind-induced mixed layer depth $\ell_{W} \simeq \frac{\kappa u_{* W}}{f}$ gives the critical friction velocity $u_{* W c} \simeq 1.2 \mathrm{~cm} \mathrm{~s}^{-1}$ for which $\ell_{W}=D_{0}$. Measurements indicate $u_{* W}>u_{* W c}$ about $23 \%$ of the time for the energetic period February to mid-May, and about $7 \%$ of the 
time for the less energetic period mid-May to August (including the tropical storms in August, e.g., Fig.4.2). These results indicate up/downwelling may occur intermittently during strong wind-events, but is not an inherent feature of the Georges Bank circulation.

Previous investigation has shown changes in heat content on the southern flank largely balance surface heating and cooling (Fig.4.7, also Lentz and Beardsley, personal communication). In 1995, exceptions occured during the SWIs in May and August, which resulted in large lateral temperature gradients and advection of the temperature field. Fig.4.8 shows the heat balance for the May SWI, when on-bank migration of the Shelf Slope front caused significant heat transport.

Although surface heating on Georges Bank is large in summer and generally not balanced by lateral advection, expression (11) predicts the observed mixed layer depth well for period II between the May and August SWIs (Fig.4.9b). In Fig.4.9, $A=1$, and $N_{I}$ was obtained from the temperature difference between the first sensor underneath the base of the mixed layer and a second sensor at the minimum distance $3 \mathrm{~m}$ below the first. Salinity was estimated using vertically interpolated coefficients from linear T-S fits to SeaCAT measurements. Least squares fits were performed on one-day intervals of hourly averaged data centered around the midpoints of the fits. Assuming one independent value every two days, the fitted slope between the daily averaged observed and predicted mixed layer depths is $1.3 \pm 0.2$ at the $95 \%$ confidence level and intersects the $y$-axis at $1.8 \pm 0.4 \mathrm{~m}$. These results are insensitive to whether or whether not a low-pass filter has been applied before the averaging. The good agreement between the observations and expression (11) indicates that the surface heat flux determines the density distribution at the base of the mixed layer. Thus, although not directly included in the derivation of (11), the stabilizing effects of surface heating reflect in estimates of $N_{I}$ (Lentz, personal communication). Expression (11) does not perform well for large parts of period I, which include numerous events of strong surface cooling (Figs.4.9a and 4.6c). Detailed investigation shows data points that are closely scattered along the line $h_{p r t}=h_{m}$ in Fig.4.9.a are largely representative of April 10-30 when 95\% of the daily averaged surface heat flux were within $162 \pm 112 \mathrm{~W} \mathrm{~m}^{-2}$. Agreement between the observed and predicted mixed layer depths is weak during the May SWI, when three-dimensional processes determined the temperature field (Fig.4.9c). 


\subsection{The Wind-Driven Circulation}

\subsubsection{Estimation of the Wind-Induced Currents}

In order to investigate the vertical structure of the wind-induced currents, barotropic and baroclinic flow contributions not directly related to wind forcing need to be removed from the measurements. In the deep ocean away from density fronts that introduce thermal wind shear, extraction of the wind-driven flow may be achieved by assuming a reference level where the stress and wind-induced circulation vanish (e.g., Price et al., 1987). Below the reference depth $z_{r}$, the circulation is in geostrophic balance with the sea surface slope and described by the velocity vector $\mathbf{V}_{0}$. Above $z_{r}$, the measured currents can be decomposed linearly into the sum of the wind-driven component $\mathbf{V}_{W}$ and the barotropic component $\mathbf{V}_{0}=\mathbf{V}\left(z_{r}\right)$. Thus, the observed velocities may be written

$$
\mathbf{V}(z)=\mathbf{V}_{W}(z)+\mathbf{V}_{0} .
$$

Applying a low-pass filter with time window long enough to suppress inertial motions and assuming subinertial acceleration is small, the upper ocean momentum balance for the wind-induced currents is described by

$$
\text { if }\left\langle\mathbf{V}_{W}\right\rangle=\frac{1}{\rho_{0}} \frac{\partial\langle\boldsymbol{\tau}\rangle}{\partial z} \text {, }
$$

where the velocity and stress vectors are in complex form $\mathbf{V}_{W}=u_{w}+i v_{w}$ and $\tau=$ $\tau^{x}+i \tau^{y}$, respectively. Under consideration of the boundary conditions $\tau=\boldsymbol{\tau}_{W}$ at the surface $(z=0)$ and $\tau=0$ at $z=z_{r}$, vertical integration of (13) gives the Ekman transport (Ekman, 1905)

$$
i \int_{z_{r}}^{0} \mathbf{V}_{W} d z=\frac{\tau_{W}}{\rho_{0} f}
$$

where angular brackets denoting the low-pass filter have been omitted. The Ekman transport is directed to the right of the wind and independent of the parameterization of the internal stress vector. 
Analytical predictions for the vertical distribution of $\mathbf{V}_{W}$ require the internal stress vector be expressed as $\frac{\boldsymbol{\tau}}{\rho_{0}}=K \frac{\partial \mathbf{V}_{W}}{\partial z}$, where $K$ is an eddy viscosity. Solutions to (13) depend strongly on the choice of $K$, i.e., on its magnitude and variation with depth. Assuming the eddy viscosity $K$ is a constant, Ekman (1905) derived the classical solution for the steady wind-driven current profile in an infinitely deep ocean

$$
\left(u_{W}, v_{W}\right)=V_{S} \exp \left(\frac{z}{D_{E}}\right)\left(\cos \left(\frac{\pi}{4}-\frac{z}{D_{E}}\right), \sin \left(\frac{\pi}{4}-\frac{z}{D_{E}}\right)\right.
$$

where similar to (14) angular brackets have been omitted. In (15), the windstress is northward in the direction of $v_{W}, V_{S}=\frac{\tau_{W}}{\rho_{0}(K f)^{1 / 2}}$ is the current amplitude at the surface, $f=0.95 \cdot 10^{-4} \mathrm{~s}^{-1}$ is the Coriolis parameter, and $D_{E}=\left(\frac{2 K}{f}\right)^{1 / 2}$ is the e-folding scale depth, also referred to as Ekman depth. Boundary conditions are $\tau=\boldsymbol{\tau}_{W}$ at the surface $(z=0)$ and $\tau=0$ at $z=-\infty$. According to (15), the current vector is $45^{\circ}$ to the right of the wind at $z=0$, and rotates clockwise for $f>0$ by $57^{\circ}$ ( 1 radian) over one e-folding depth.

In coastal regions such as Georges Bank, determination of the wind-driven currents is more difficult than for the deep ocean. Complications arise in particular from the shallow water depth, the presence of a coastal boundary, and density fronts. In winter, large surface winds can cause significant stress divergence everywhere in the water column, implying the choice of a reference level with zero shear is not appropriate. During the warm season, increasing lateral stratification enhances thermal wind shear, and decomposition of the velocity vector according to (12) is no longer sufficient. In addition, the nonlinearity of the flow field introduces advective terms which are generally not considered in deep ocean studies.

Here, separation of the Ekman currents from the background flow field is attempted by rewriting the total velocity vector $\mathbf{V}$ in the form

$$
\mathbf{V}(z)=\mathbf{V}_{0}+\mathbf{V}_{\nabla \rho}(z)+\mathbf{V}_{W}(z)
$$

where $\mathbf{V}_{0}$ is a barotropic velocity, and $\mathbf{V}_{\nabla \rho}$ is in geostrophic equilibrium with the lateral density gradients. Provided $\mathbf{V}_{0}$ and $\mathbf{V}_{\nabla \rho}$ are known, (16) can be solved for $\mathbf{V}_{W}$. Underlying (16) is the assumption that thermal wind and wind 
mixing are the only mechanisms causing vertical shear, while all other contributions to the subtidal flow field are barotropic. Obviously, this approximation holds only to lowest order, since bottom friction adds to the vertical structure of the subtidal currents. Results from sections 4.2 .4 and 4.2 .6 have shown the bottom stress is not a dominant term in the time-averaged momentum balance (Table 4.1) and is about one order of magnitude smaller than the wind stress during times of energetic winds (Fig.4.5). Time-mean magnitudes of the kinematic bottom stress are about $0.3-0.4 \mathrm{~cm}^{2} \mathrm{~s}^{-2}$ during periods I and II, giving representative bottom boundary layer thicknesses $l_{b}=\frac{\kappa u_{* b}}{f}=23-27 \mathrm{~m}$ for the subtidal flow. Thus, bottom frictioninduced vertical shear is mostly limited to the lower $30-35 \%$ of the water column, while the current distribution at greater heights is affected by other processes.

Estimation of the baroclinic term $\mathbf{V}_{\nabla \rho}$ from data is straight forward. Taking $z$ positive upward, the thermal wind equations are

$$
\begin{aligned}
& \frac{\partial v}{\partial z}=-\frac{g}{f} \frac{\partial \rho}{\partial x} \\
& \frac{\partial u}{\partial z}=\frac{g}{f} \frac{\partial \rho}{\partial y}
\end{aligned}
$$

Density gradients can be computed from measurements at the ST1, ST2, and SF moorings, and interpolated with depth to obtain estimates at mid-depths between adjacent current meters. Integrating (17) from the surface $(z=0)$ downward gives estimates of $\mathbf{V}_{\nabla \rho}$ at the current meter depths, where the integration constant $\mathbf{V}_{\nabla \rho}(z=0)=0$.

Meaningful values of $\mathbf{V}_{0}$ are difficult to determine, since no measurements were taken that would allow for the direct evaluation of the sea surface slope. Two different approaches were used to estimate $\mathbf{V}_{0}$ during conditions representative of winter (method I) and summer (method II).

Method I: During period I, large surface winds combined with weak vertical stratification resulted in a current response that extended throughout most of the water column, thus limiting the choice of a reference level with zero shear. Hence, the approach used in deep ocean studies could not be applied here, i.e., $\mathbf{V}_{0}$ could not be determined from the velocities measured at some reference depth. To obtain estimates of the barotropic circulation, the momentum equations were written as 


$$
-g \nabla \eta-[\mathbf{N}]=i f[\mathbf{V}]+[\mathbf{B}]-\frac{\boldsymbol{\tau}_{W}}{\rho_{0} D}+\frac{\tau_{b}}{\rho_{0} D}+\left[\frac{\partial \mathbf{V}}{\partial t}\right]
$$

where all vectors are in complex form, $\mathbf{N}=\frac{\partial\left(u^{2}\right)}{\partial x}+i \frac{\partial(u v)}{\partial x}, \mathbf{B}=\frac{g}{\rho_{0}}\left(\frac{\partial \rho}{\partial x}+i \frac{\partial \rho}{\partial y}\right)(D+z)$, and square brackets denote the depth-average as in (6). The barotropic velocity was then defined as

$$
\mathbf{V}_{0}=-\frac{1}{i f}(g \nabla \eta+[\mathbf{N}])
$$

and determined by estimating the right hand side of (18) from data and substituting the result into (19). Alternatively, $[\mathbf{N}]$ can be estimated from the measurements as suggested by (10), and moved from the left to the right hand side of (18). Taking this approach gives $\mathbf{V}_{0}=-\frac{g}{f} \nabla \eta$ instead of (19). Here, definition (19) was applied, since it is difficult to obtain reliable estimates of $[\mathbf{N}]$ given the limited vertical resolution of the current measurements at ST2 and the large horizontal distance between the ST1 and ST2 moorings.

Substitution of (16) and (19) into (18) yields after integration over the water depth

$$
i \int_{-D}^{0} \mathbf{V}_{W} d z=\frac{\boldsymbol{\tau}_{W}}{\rho_{0} f}-\frac{\boldsymbol{\tau}_{b}}{\rho_{0} f}-\frac{1}{f} \int_{-D}^{0} \frac{\partial \mathbf{V}}{\partial t} d z
$$

Expression (20) illustrates that by definition, deviations of the transport estimates $i \int_{-D}^{0} \mathbf{V}_{W} d z$ from the Ekman transport $\frac{\boldsymbol{\tau}_{W}}{\rho_{0} f}$ are due to the bottom friction and acceleration terms. As indicated by Table 4.1 and Fig.4.5, the contribution of these terms to the subtidal momentum balance is generally small, so that (20) should approach (14) to lowest order.

Method II: A second, different approach was used for the strongly stratified period II, when winds were about four times less energetic than during period I. Method II is based on the observation that after the thermal wind shear has been removed from the measurements, low-pass filtered velocity profiles representative of summer often display a strongly sheared region near the surface, followed by an 
interior flow regime with little vertical variation and a bottom boundary layer of about 30-m thickness. The depth of the wind-induced shear layer varied depending on wind-stress and stratification, and was typically no larger than $20 \mathrm{~m}$. An example is shown in Fig.4.10 for the wind event June 27-29. Thermal wind shear was weak at the end of June since lateral density gradients were small during this time (Fig.4.4). The current distribution displays strong shear in the upper $19 \mathrm{~m}$, approximately corresponding to the depth of the mixed layer base at the peak of the event (Fig.4.10a-c). The distribution of $R i<R i_{c}\left(R i_{c}=0.5\right)$ indicates wind-induced current shear in the upper water column coincided with intense mixing, followed by a region of little turbulence at mid-depth and a highly turbulent bottom boundary layer in the lower $\approx 30 \mathrm{~m}$ (Fig.4.10d). Richardson numbers were computed from stratification estimates based on hourly averaged salinity and temperature data, with salinity at the TPOD-depths derived from T-S fits as described in section 4.2.7. The temperature sensors used for the fits were chosen such that their vertical distance was $>3 \mathrm{~m}$. Velocity shear was computed from hourly averaged, unfiltered current measurements and interpolated linearly with depth to obtain estimates at mid-depth between the temperature sensors. Velocities estimates above 5-m depth were obtained from linear extrapolation of measurements at greater depth. Error bars in Fig.4.10d are from Monte Carlo simulations as described in section 2.4.3. $R i_{c}=0.5$ was chosen as a critical Richardson number, since application of the criterion for linear instability, $R i_{c}=0.25$, requires the Ozmidov scale to be resolved by the measurements. Rough scaling indicates that this is not the case: microstructure data taken on June 11-12 suggest $\epsilon \approx 10^{-8} \mathrm{~m}^{2} \mathrm{~s}^{-3}$ for $N^{2} \approx 2 \cdot 10^{-4} \mathrm{~s}^{-2}$ at $10-\mathrm{m}$ depth (measurements taken by Oakey and Hebert, see also GSO Rhode Island Tech. Report 96-6). For the event presented here, time-mean buoyancy frequencies in the main thermocline were about $2 \cdot 10^{-4} \mathrm{~s}^{-2}$. Using $\epsilon \approx 10^{-8} \mathrm{~m}^{2} \mathrm{~s}^{-3}$ as a rough approximation for the dissipation rate near the surface, the Ozmidov scale defined in 2.4 .3 is $<1 \mathrm{~m}$. Although the choice $R i_{c}=0.5$ is somewhat arbritrary, results are relatively insensitive to the exact specification of $R i_{c}$. Taking $R i_{c}=0.25$ or $R i_{c}=1.0$ gives percentage distributions of $R i<R i_{c}$ that are similar to Fig.4.10d, with differences that are less $<10 \%$ in the upper water column.

To identify the region of wind-induced current shear as a function of time, the $R i$-number analysis presented in Fig.4.10d was performed on a daily basis, i.e., for time-windows of $24 \mathrm{hrs}$ length. The centerpoint of the time-window was passed 
through period II such that one profile was obtained every hour. The smallest depth where $R i<R i_{c}$ less than $10 \%$ of the 24 -hour interval was taken to represent the vertical extent of the wind-driven circulation. In cases where the number of events $R i<R i_{c}$ did not fall below $10 \%$ anywhere in the upper $40 \mathrm{~m}$, the cut-off criterion was increased in steps of $1 \%$ until the condition was met. The first current meter below the inferred reference level was used to determine $\mathbf{V}_{0}=\mathbf{V}-\mathbf{V}_{\nabla \rho}$. About $99 \%$ of the computed reference depths $h_{r}$ were between 8-29 m, so that the four VMCMs spanning the 10-31-m depth-range were most commonly used to estimate $\mathbf{V}_{0}$. Fig.4.11 indicates the reference level obtained from $R i$-analysis is significantly deeper than the vertical extent of the mixed layer $h_{m}$. Assuming one independent estimate every two days, least squares fitting the daily averaged values $h_{m}$ to $h_{r}$ gives $h_{r}=(1.3 \pm 0.7) \cdot h_{m}+(10.6 \pm 1.5) \mathrm{m}$ at the $95 \%$ confidence level. These results are insensitive to whether or not the low-pass filter has been applied before the averaging. The estimated $10.6 \pm 1.5-\mathrm{m}$ offset of $h_{r}$ with respect to $h_{m}$ indicates mixing extends beyond the homogeneous surface waters into the stratified interior and occurs even when $h_{m}=0$.

Estimation of the wind-driven currents as described above involves several sources of potentially large error. For example, uncertainties are introduced by the estimation of density gradients and resulting thermal wind shear, definition of a reference level in summer, and computation of the barotropic currents from indirectly determined sea surface gradients and depth-averaged advection terms in winter. Quantification of the combined error is impossible, since the uncertainties due to the various error sources are not known. Some indication of how closely the velocities $\mathbf{V}_{W}$ deduced from data represent the wind driven circulation can be obtained by comparing the left and right hand sides of (14). For method I, expression (20) demonstrates that deviations between the observed transport $i \int_{-D}^{0} \mathbf{V}_{W} d z$ and the predicted Ekman transport $\frac{\tau_{W}}{\rho_{0} f}$ are explained by the presence of bottom friction and temporal changes of the subtidal flow. Additional differences between the right and left hand sides of (20) are associated with the details of the interpolation procedure, i.e., interpolation of the thermal wind to mid-depth between adjacent VMCMs to obtain $\mathbf{V}_{\nabla \rho}$ versus interpolation to the SeaCAT depths when estimating $[\mathbf{B}]$. However, such differences are small, so that comparison of $i \int_{-D}^{0} \mathbf{V}_{W} d z$ to $\frac{\boldsymbol{\tau}_{W}}{\rho_{0} f}$ is 
an effective measure to quantify the contribution of bottom stress and acceleration terms to estimates of $\mathbf{V}_{W}$. In summer, when method II is used to determine the barotropic circulation, (20) no longer represents an identity for the following reasons: First, $V_{0}$ is determined from the flow profile rather than by closing the momentum balance using (18) and (19), and second the integration depth is no longer equal to the water depth but corresponds to $z_{\tau}$. Hence, comparison of the left and right hand sides of (14) represents an independent check of how well the estimated wind-driven transport agrees with the predicted Ekman transport, i.e., how well the wind-driven currents were estimated from data.

\subsubsection{Winter Average}

The long-term average of the wind-induced currents is examined following the "coherent ensemble average" method outlined by Price et al. (1987). Subtidal wind stress and current measurements were vector-averaged over each day, and rotated into a coordinate system where the wind vector points arbitrarily north. The rotated system follows low-frequency variations in wind direction, allowing for analysis of the low-passed currents in a wind-relative frame of reference. Taking the ensemble average of all daily rotated vectors over a chosen period gives the time-mean windinduced current response. The winter investigation period extends from February 1 to March 11 (period IA), and defines a sub-period of the previously discussed period I (Fig.4.12). Period IA was chosen for analysis, since it presents a time of large winds as well as extremely weak vertical stratification (Fig.4.12a,b). Typical buoyancy frequencies squared were $N^{2} \approx 10^{-5} \mathrm{~s}^{-2}$ at mid-depth and $N^{2} \approx 2 \cdot 10^{-6} \mathrm{~s}^{-2}$ above and below the weak winter pycnocline (Fig.4.13). Intermittent intrusions of low salinity water covered about $12 \%$ of the investigation period and caused brief events with $N^{2}=O\left(10^{-4}\right) \mathrm{s}^{-2}$ near the surface.

Fig.4.14 shows the ensemble-averaged, wind-induced currents veer cum sole with depth, as suggested by theory. The clockwise rotation of the current vector is particularly obvious in the upper $19 \mathrm{~m}$. At larger depths, the estimated speeds are small $\left(\approx 1 \mathrm{~cm} \mathrm{~s}^{-1}\right)$, and the direction of the velocity vectors is extremely susceptible to the accuracy of the estimation method. Vertical integration of the wind-driven currents gives a transport vector that is within $14 \%$ of the ensemble-averaged Ekman transport $\frac{\bar{\tau}_{W}}{\rho_{0} f}$ and about $86^{\circ}$ to the right of the wind, in good agreement with (14) (Fig.4.14). The integration depth was chosen to equal the water depth, since 
scaling arguments reveal wind-mixing extended throughout most or all of the water column (section 4.2.6). The ensemble averaged bottom stress roughly opposes the wind stress and amounts to about $10 \%$ of the wind stress' magnitude. Closer investigation shows the remaining deviation between the predicted and estimated Ekman transport is largely explained by the unsteadiness of the subtidal flow due to acceleration perpendicular to the wind stress, and to some degree by the details of the vertical interpolation chosen to determine the thermal wind currents.

Although compass uncertainties prevent definite conclusions about the rotation of the current vector with depths, measurements indicate the rotation rate is about twice as large between the VMCMs at 5 and 10-m depth than between those at 10 and 19-m depth (Fig.4.14). The change in rotation rate is caused by strong downwind shear between the upper two current meters, which exceeds the shear estimates at greater'depth by more than a factor of two. A likely explanation for this behavior is wave-bias, which results from movement of the surface buoy with the free surface. Schudlich and Price (1998) (hereafter SCHP) found wave bias enhances the velocity shear in the downwind direction, corresponding to the preferred orientation of phase propagation of wind-induced surface waves. From geometrical considerations and linear wave theory, it can be shown that wave-bias is largest near the surface, and decreases with depth on an e-folding scale proportional to the wave length (Santala, 1991). Thus, the near-surface measurements are particularly affected by wave-induced downwind current shear. Estimation of the wave bias requires knowledge of spectral wave data, which is not available for this study. Santala (1991) found typical wave bias due to the buoy's vertical motion was of order 1-3 $\mathrm{cm} \mathrm{s}^{-1}$ at $4 \mathrm{~m}$-depth during the Shelf Mixed Layer Experiment (SMILE) on the northern California shelf. Wave conditions during SMILE were similar to those during period IA, when the temporal mean and standard deviation of the significant wave height amounted to $2.9 \pm 1.4 \mathrm{~m}$. Since Fig.4.14 presents data averaged over times of large as well as moderate winds (Fig.4.12a), the time-mean effects of wave-bias may be smaller than for individual, large wind-events.

The results presented in Fig.4.14 are qualitatively similar to earlier observations made by SCHP as part of the Long-Term Upper Ocean Study (LOTUS). SCHP examined the response of the near-surface currents to wind forcing at a location about $500 \mathrm{~km}$ west of Bermuda in more than $5000-\mathrm{m}$ water depth. For conditions representative of winter and time-mean wind stress magnitudes comparable to those 
during period IA, SCHP found the current vector was about $43^{\circ}$ to the right of the wind stress at 5 -m depth, and veered clockwise by $\approx 36^{\circ}$ over the e-folding scale of the velocity amplitudes $\left(L_{e}=25 \mathrm{~m}\right)$. In the present case, the angle between the wind stress and currents $5 \mathrm{~m}$ below the surface is $\approx 34^{\circ} \pm 5^{\circ}$, where $\pm 5^{\circ}$ corresponds to the compass uncertainty of the VMCMs. Excluding the topmost current meter due to the possibly large effects of wave-bias, the mean e-folding scale of the velocity amplitudes above 19-m depth is $L_{e} \approx 12 \mathrm{~m}$. Over the same depth-range, the velocity vector rotates by about $30^{\circ}$.

SCHP's results were inconclusive about whether the observations correctly described the velocity distribution in winter, or whether wave-bias greatly increased the downwind current shear in the upper $\approx 15 \mathrm{~m}$. Since the observed transport vector displayed a large downwind component in SCHP's case, it is likely wave bias played a significant role in the upper water column. Here, the downwind component of the estimated transport vector is forced to be small, since (20) represents an identity by definition. Thus, small downwind transport does not necessarily imply the absence of strong wave-bias in Fig.4.14. However, the observation that downwind shear of the wind-induced currents is more than twice as large between the upper two VMCMs than at greater depth is independent of (20) and indicates the time-averaged effects of wave bias may be significant only at the topmost current meter. Thus, results at $7.5-\mathrm{m}$ depth and below may be more true to the actual distribution of $\mathbf{V}_{W}$ than in SCHP's case.

Based on measurements between 7.5-19-m depth, the average rotation rate extrapolates to give the 1 -radian $\left(57^{\circ}\right)$ turning depth $L_{\theta} \approx 22 \mathrm{~m}$, where again the topmost current meter has been excluded from the analysis to account for wave bias. Defining the "flatness" parameter $F=\frac{L_{\theta}}{L_{e}}$ (Price and Sundermeyer, submitted; hereafter PS) and using the e-folding scale $L_{e}=12 \mathrm{~m}$ listed above yields $F \approx 1.8$. By definition, $F=1$ for expression (15), so that the flatness of the observed spiral is about twice the value suggested by Ekman theory.

Solving (15) for the Ekman depth $D_{E}=L_{\theta}=22 \mathrm{~m}$ and the ensemble-averaged wind stress magnitude $\frac{\bar{\tau}_{W}}{\rho_{0}}=1.4 \mathrm{~cm}^{2} \mathrm{~s}^{-2}$ gives a current spiral that is about $24^{\circ} \pm 5^{\circ}$ to the right of the observations at 5 - $\mathrm{m}$ depth, and $12^{\circ} \pm 5^{\circ}$ to the right of the observations at 7.5-19 m depth (Fig.4.14). The predicted current speeds approach zero near the bottom, indicating the assumption of an infinitely deep ocean in (15) is appropriate to lowest order. In the upper $19 \mathrm{~m}$ of the water column, model solutions 
of $\mathbf{V}_{W}$ are 8-60\% larger than the estimates inferred from data, with increasing overestimation toward greater depth.

Deviations of the estimated currents $\mathbf{V}_{W}$ from (15) are not surprising, since it has long been understood that classic Ekman theory does not capture the full physics of the flow field. In particular, the vertical structure of (15) depends critically on the assumption of constant $K$, which represents a very simplified parameterization of turbulent mixing. Many studies have been aimed at determining more realistic eddy viscosity distributions than the constant $K$-distribution incorporated in the Ekman model. Suggestions range from relatively simple analytical models that prescribe the $K$-profile to complicated higher order turbulence closure schemes. For example, Madsen (1977) solved the upper ocean momentum balance assuming a logarithmic layer near the free surface in analogy to bottom boundary layer theory. Using an eddy viscosity that increases linearly with depth as specified by $K=\kappa u_{* W}|z|$, Madsen (1977) predicted the wind-induced currents as a function of wind stress. According to Madsen's (1977) model, the current shear is almost entirely downwind in the upper few meters in agreement with the idea of a constant stress layer near $z=0$. Fig.4.14 shows the Madsen solution applied to the present case for depths $>0.3 \mathrm{~m}$. Velocities above $0.3-\mathrm{m}$ depth are not shown and reach about $30 \mathrm{~cm} \mathrm{~s}^{-1}$ close to the surface $(z=O(\mathrm{~mm}))$. As opposed to the classical Ekman solution, Madsen's (1977) model underpredicts the observed current magnitudes and underestimates the rotation rate below $5-\mathrm{m}$ depth. The flatness of the computed current spiral is about 3-4 between 5-19 m depth, and thus significantly larger than suggested by observation.

In disagreement with Madsen's (1977) analytical model, Santala (1991) found velocity profiles display little shear in the top few meters of the water column, a behavior consistent with the idea of an intensely mixed wave zone as described by Kitaigorodskii et al. (1983). The existence of a wave zone opposes the assumption $K=\kappa u_{* W}|z|$ near the surface, since eddy viscosities in such a highly turbulent wave region would be large rather than approach zero. Another completely different approach aimed at describing wind-induced turbulence was developed by PS and is based on the observation that the mean shear and stress vectors are not always parallel. From their data, PS concluded a complex eddy viscosity may present a suitable parameterization of turbulent mixing.

Mixing is also affected by winter cooling, which can cause convection over pe- 
riods of days to weeks (Fig.4.12c). The impacts of convective effects on the $K$ distribution are not well understood, and thus represent another unknown when modeling the wind-driven circulation. In summary, none of the simplistic models shown in Fig.4.14 can be expected to fully describe the processes that determine the vertical distribution of the wind-induced flow.

\subsubsection{Winter Event Analysis}

To determine whether the observed velocity structure is more than a result of the long-term averaging, the wind-induced currents are examined for three energetic wind events. The event analysis covers the 62,33 and 29 hrs-long periods on February 5-8 (W1), February 12-13 (W2) and April 5-6 (W3), respectively (Fig.4.15). Estimation of the wind-induced currents followed the procedure outlined above, with the difference that the average was taken over the duration of each event, and the direction of the event averaged wind vector was defined as "north". During these events, the kinematic wind stress reached peak values between 4-7 $\mathrm{cm}^{2} \mathrm{~s}^{-2}$ (Fig.4.15, top). Time-windows were chosen such that $\frac{\tau_{W}}{\rho_{0}}>2 \mathrm{~cm}^{2} \mathrm{~s}^{-2}$ and the wind direction was uniform to less than $25^{\circ}$ (Fig.4.15, middle).

The events W1-3 coincided with periods of strong surface cooling, suggesting convective processes acted together with tidal and wind mixing to homogenize the water column (Fig.4.15, bottom). Measurements show the vertical temperature distribution was uniform within $0.02^{\circ} \mathrm{C}$ during W1 and W2 (Fig.4.16, top). During W3, thermal stratification was $<0.01^{\circ} \mathrm{C}$ between the surface and $45-\mathrm{m}$ depth, and greater at larger depth. Salinities display similar behavior, indicating density was nearly homogeneous for W1 and W2, and weakly stratified $\left(N^{2} \approx 0.2 \cdot 10^{-4} \mathrm{~s}^{-2}\right)$ at depth for W3 (Fig.4.16, middle and bottom).

The vertical structure of the time-mean wind-induced currents is qualitatively similar for W1-3 (Fig.4.17), and bears strong resemblance to the ensemble averaged results shown in Fig.4.14. Transport estimates are within 6-14\% of $\frac{\bar{\tau}_{W}}{\rho_{0} f}$ and 95-102 ${ }^{\circ}$ to the right of the wind. All events display a region of nearly constant veering between $7.5-19 \mathrm{~m}$ below the surface, with the characteristic rotation depths $L_{\theta} \approx 15 \mathrm{~m}, L_{\theta} \approx 14 \mathrm{~m}$, and $L_{\theta} \approx 19 \mathrm{~m}$ for W1, W2, and W3, respectively. Averaged over the topmost $10 \mathrm{~m}$, clockwise rotation of the velocity vector for $\mathrm{W} 1, \mathrm{~W} 2$, and W3 is about $15 \%, 30 \%$ and $100 \%$ larger than the veering at $10-19-\mathrm{m}$ depth. As discussed in the previous section, this behavior may be caused by downwind shear 
due to wave-bias near the surface. The e-folding scale depth $L_{e}$ is about $13 \mathrm{~m}$ for $\mathrm{W} 1,12 \mathrm{~m}$ for $\mathrm{W} 2$, and $18 \mathrm{~m}$ for W3. Estimates of $L_{\theta}$ and $L_{e}$ give the flatness parameters $F=1.1-1.2$, in good agreement with (15).

Similar to results for period IA, using $D_{E}=L_{\theta}$ in expression (15) predicts the wind-driven currents are to the right of the observations and exceed the current speeds deduced from data (Fig.4.17). The Ekman solutions are 7-30 clockwise of the measurements, and have magnitudes about twice as large as suggested by data. Also similar to the ensemble-averaged case, Madsen's (1977) eddy viscosity model underestimates the observed velocities and produces less veering than indicated by the measurements.

Results of the event analysis show the following features are inherent to the three cases presented here: First, the flatness of the observed current spirals $F=$ 1.1-1.2 is in good agreement with Ekman theory. Second, predictions from Ekman theory give current speeds that are about twice as large as those inferred from measurements. Third, all Ekman solutions are to the right of the observations, i.e., the predicted spiral is rotated clockwise by about $10-30^{\circ}$. Although none of these properties has an apparent physical explanation, their applicability to all three wind events is intriguing. In particular the observed flatness indicates that (15) describes the distribution of the unit velocity vector with reasonable accuracy, where the term "unit vector" refers to the estimated wind-driven currents divided by the surface amplitude $V_{S}=\frac{\bar{\tau}_{W}}{\rho_{0}(K f)^{1 / 2}}$.

It has been pointed out in the previous section that the vertical structure of the wind-driven currents depends strongly on the specification of $K$. Although it is not possible to infer a meaningful $K$-distribution from the data presented here, it is reasonable to assume that $K$ is significant throughout most of the water column for W1-3. The rationale behind this conclusion is that surface heat loss occurred during all three events, suggesting that free convection played a major role in homogenizing the water column. Convective mixing differs from mechanical turbulence in that mixing happens through convective overturning rather than sheardriven turbulent motion. The vertical scale of the convection cells is limited by buoyancy forces, and may cover the entire water depth if the surface cooling is strong and the background stratification weak. In contrast, the diameter of friction-induced eddies cannot exceed the distance from the nearest boundary, giving rise to eddy viscosity parameterizations with pronounced vertical structure such as Madsen's 
(1977) $K$-profile that increases linearly away from the surface.

Despite the presence of surface cooling, the assumption of relatively homogeneous $K$ may not be appropriate in the upper few meters where wind-induced turbulence is most intense. However, numerical experiments show the details of the eddy viscosity parameterization immediately below the surface do not significantly affect velocity predictions at those depths where measurements are available. For example, matching $K=\kappa u_{*} \dot{W}|z|$ near $z=0$ to $K=\frac{D_{E}^{2} f}{2}$ in the region below gives results very similar to the Ekman solutions at the instrumentation depths (not shown), where $D_{E}=L_{\theta}$ was taken from observation. With $L_{\theta}$ ranging from 14-19 $\mathrm{m}$ for W1-3, $K=\frac{D_{E}^{2} f}{2} \approx 0.01-0.02 \mathrm{~m}^{2} \mathrm{~s}^{-1}$, and the region of linearly increasing $K$ extends over 1-2.5 m. Significant differences between model solutions for linear-constant and constant $K$-distributions are limited to the upper $5 \mathrm{~m}$ or less. Similarly, representing intense wave mixing by $K=1$ between 0 and $5-\mathrm{m}$ depth produces homogeneous flow in the wave zone, but hardly affects the solutions at greater depth.

In summary, it is possible that convective overturning results in a $K$-distribution that is more uniform with depth than for the case of strictly mechanical mixing. It is also conceivable that convection is responsible for the observed similarities in the vertical structure of the wind-driven currents during W1-3. The long-term analysis in the previous section covered periods of net cooling as well as heating, so that the ensemble-averaged currents were less susceptible to the impacts of convective mixing. Thus, the idea of a relatively uniform $K$-distribution may be less applicable to the winter average than to individual cooling events. Greater depth-variation of the time-mean eddy viscosity as opposed to a more homogeneous distribution during convective events may relate to the observed larger flatness of the ensemble-averaged currents compared to results for W1-3.

It should be remarked that even in the presence of the homogenizing effects of convective overturning, observations indicate that the eddy viscosity distribution is not completely depth-independent. This follows from the comparison of the estimated velocity amplitudes to predictions from the Ekman model, which clearly indicate the assumption of constant $K$ does not fully explain the measurements. The exact nature of mixing processes due to convection and their impact on the vertical structure of the wind-driven currents demands to be investigated in future studies. 


\subsubsection{Summer Average}

To examine the wind-driven circulation during strongly stratified conditions, the "coherent ensemble average" was taken for the periods May 29-June 30 (period IIA) and July 1-August 4 (period IIB). Estimation of the wind-induced flow followed the procedure in method II outlined in section 4.3.1. Period II was divided into two subintervals to separate times before and after the rapid increase of the near-surface stratification at the beginning of July (Fig.4.18). Closer investigation shows large thermal and density stratification developed immediately below the surface (1.5-6.0$\mathrm{m}$ depth) during the last 10 days of June, and was interrupted by the wind event June 27-29 (Fig.4.19). In the latter half of June as well as throughout the month of July, increasing stratification was associated with periods of calm winds and large surface heat flux (Fig.4.18).

Fig.4.20 shows the influence of wind forcing on the velocity distribution was clearly limited to the upper 14-19 $\mathrm{m}$ (7.5-10 m) during period IIA (period IIB). The temporal mean and standard deviation of the significant wave height were about $1.4 \pm 0.8 \mathrm{~m}(1.1 \pm 0.4 \mathrm{~m})$, in comparison to $2.9 \pm 1.4 \mathrm{~m}$ for the winter analysis period IA. Thus, the time-mean effects of wave-bias on the near-surface currents should be less in summer than in winter. Estimates of the wind-induced transport were obtained from vertical integration of $\mathbf{V}_{W}$ between the surface and the reference level used to determine $\mathbf{V}_{0}$. Hence, the integration depth varied with time according to changes in reference depth. Similar to the current estimates, the daily means of the transport vector were rotated into a wind-relative coordinate system before their ensemble average was taken. The ensemble-averaged transport vectors show good agreement with theory. Their magnitudes are within $22 \%(4 \%)$ of $\frac{\bar{\tau}_{W}}{\rho_{0} f}$ and their direction is about $86^{\circ}\left(102^{\circ}\right)$ to the right of the wind stress for period IIA (IIB) (Fig.4.20).

The vertical structure of the wind-induced currents varies between periods IIA and IIB. For period IIA, the mean e-folding depth of the current magnitudes in the top $14 \mathrm{~m}$ is $L_{e} \approx 4 \mathrm{~m}$. The 1-radian rotation scale $L_{\theta}$ is difficult to determine, since the flow directions at various depths are hardly distinguishable within the $\pm 5^{\circ}$ compass uncertainties (Fig.4.20a). Ignoring the error limits imposed by the compasses, a rough estimate based on observations at the upper three VMCMs is $L_{\theta} \approx 26 \mathrm{~m}$, yielding the flatness parameter $F \approx 6.5$. However, these values 
should be approached with caution, since for a surface-trapped velocity response like the one observed here, even the small $4^{\circ}$ deviation of the transport vector from the predicted right angle may indicate the downwind component at the upper current meter is overestimated. Thus, it cannot be ruled out that the wind-driven currents are more unidirectional than suggested by Fig.4.20a. For period IIB, $L_{e} \approx$ $5 \mathrm{~m}$ between 5 - and $10-\mathrm{m}$ depth. The veering of the velocity vector cannot be distinguished from the compass error, so that $F \rightarrow \infty$ '(Fig.4.20b). Because of the flat appearance of the current spiral during periods IIA and IIB, the agreement with the Ekman solution is poor (not shown). Results from Madsen's (1977) model also show little resemblance to the measurements (not shown).

Comparison of Fig.4.14 to Fig.4.20 shows the clockwise rotation of the velocity vector with depth is significantly more pronounced for the winter than the summer results. Observations representative of winter give $F \approx 1.8$ for the ensemble average (Fig.4.14), and $F=1.1-1.2$ for individual wind events (Fig.4.17). Thus, the flatness of the current spiral increased as strong solar radiation caused surface cooling to disappear and heating to occur, the seasonal thermocline developed, and the wind-induced currents became more and more surface-trapped. Figs.4.13 and 4.21 illustrate the changes in vertical stratification between periods IA, IIA, and IIB. While the time-mean $N^{2}$ was $O\left(10^{-6}-10^{-5}\right) \mathrm{s}^{-2}$ in the upper water column for period IA, corresponding values for period IIA and IIB were $O\left(10^{-4}-10^{-3}\right) \mathrm{s}^{-2}$. Vertical stratification in the top $\approx 10 \mathrm{~m}$ was about four times greater during period IIB than IIA due to enhanced thermal stratification as time progressed toward late summer (Fig.4.21). This observation together with the fact that the time-mean wind stress was almost twice as large for period IIA than IIB explains why the wind-induced flow is limited to about one half the depth in Fig. $4.20 \mathrm{~b}$ compared to Fig.4.20a.

The flat appearance of the current spiral during stratified conditions is supported by earlier results described in SCHP. For conditions representative of summer and wind stress magnitudes similar to those during period II, SCHP demonstrated the ensemble-averaged, wind-driven currents were about $78^{\circ}$ to the right of the wind at 5 -m depth, and veered by $\approx 20^{\circ}$ over the e-folding scale $L_{e} \approx 12 \mathrm{~m}$. SCHP's results strongly resemble the observations for period IIA shown in Fig.4.20a, which give an angle of $82 \pm 5^{\circ}$ between the wind stress and currents at 5-m depth, and clockwise rotation of the velocity vector by about $\approx 10^{\circ}$ over one e-folding scale $\left(L_{e} \approx\right.$ 
$4 \mathrm{~m}$ ). Results for period IIA also display qualitative agreement with observations by Chereskin (1995), who found $F \approx 2$ in the California current averaged over the 6-month period April-October, 1993.

In their investigation of stratified Ekman layers, PS and Price et al. (1986) (hereafter PWP) discuss the mechanisms that are responsible for the vertical distribution of the wind-induced circulation inferred from deep ocean studies. Their results indicate the observed spiral flatness is a consequence of the long-term averaging in the presence of vertical stratification and diurnal cycling. In the following, the effects of temporal variations in surface heat flux, density distribution and wind field are discussed according to their occurrence on diurnal and larger-than-diurnal time scales.

\subsubsection{Diurnal Cycling}

For the open ocean, Price et al. (1987) demonstrated that the vertical structure of the ensemble-averaged wind-driven circulation resembles a spiral, although instantaneous current profiles may show a different distribution. Their conclusions are based on measurements and model results presented by PWP for a region approximately $400 \mathrm{~km}$ west of San Diego. PWP's observations indicate there is little velocity shear in the surface mixed layer at all times of the day. Underneath the homogeneous surface waters, PWP found a region of significant shear and clockwise rotation of the current vector with depth. At night-time, the mixed layer deepened due to convection and the velocity structure became increasingly slablike. The diurnal variation of the mixed layer thickness together with wind-induced inertial oscillations produced a current response whose direction and vertical extent changes throughout the course of $24 \mathrm{hrs}$. Taking the ensemble average over several diurnal cycles gave a current spiral in which the velocity vector veered clockwise $(f>0)$ with depth.

In the present case, the low-pass filter suppresses diurnal as well as inertial oscillations of the flow field. Nevertheless, such oscillations may affect the ensembleaveraged currents. For diurnal variation, this can be easily demonstrated. Nighttime increase of the mixed layer thickness allows for wind-induced flow at greater depth than during the day-time hours. As a consequence, the 24-hrs average of the current vector has a mean velocity component underneath the base of the day-time mixed layer. Price et al. (1987) show ensemble-averaging the day- and night-time currents separately gives a flow distribution that is more surface-trapped during the 
day when stable stratification limits the vertical extent of the mixed layer than at night when the mixed layer deepens.

In the present case, separate ensemble averages of the day- and night-time currents are not significantly different from Fig.4.20 for both periods IIA and IIB (not shown), despite the fact that $Q$ varies strongly throughout the course of one day (Fig.4.22). Cross spectra between unfiltered estimates of the mixed layer thickness and net surface heat flux representative of period II show significant coherence at the $95 \%$ level ( $c_{95}=0.79$ ) in the diurnal frequency band, indicating the mixed layer depth responds to diurnal cycling. However, diurnal variation of the mixed layer thickness is small at ST1 compared to the case described by PWP. Averaged over period IIA (IIB), characteristic differences between the day- and night-time mixed layers are about $2.0 \mathrm{~m}(1.0 \mathrm{~m})$ (Fig.4.23a,b, bottom), as opposed to $>30 \mathrm{~m}$ for the PWP case. Thus, the effects of diurnal cycling on the wind-induced flow are small, so that the ensemble-averaged velocity distributions are similar for the dayand night-time currents.

Although the wind stress magnitudes for the PWP study and period II were approximately comparable, there are two reasons explaining why diurnal variation of the mixed layer depth was significantly smaller on Georges Bank than off the Southern Californian shelf. First, heat loss occured during no more than half of the diurnal cycles constituting periods IIA and IIB, and the heat flux averaged over all night-time cycles was above zero (Figs.4.22, and $4.23 \mathrm{a}, \mathrm{b}$, top). The majority (95\%) of night-time cooling events did not exceed $-75 \mathrm{~W} \mathrm{~m}^{-2}\left(>-100 \mathrm{~W} \mathrm{~m}^{-2}\right)$ during period IIA (IIB), and had mean values around $-33 \mathrm{~W} \mathrm{~m}^{-2}\left(-41 \mathrm{~W} \mathrm{~m}^{-2}\right)$. In comparison, PWP's estimates of night-time cooling were consistently more than $-100 \mathrm{~W} \mathrm{~m}^{-2}$ and reached values as large as $-300 \mathrm{~W} \mathrm{~m} \mathrm{~m}^{-2}$. Second, day-time stratification was strongly surface-trapped in the PWP case, with $N^{2}=O\left(10^{-4}\right) \mathrm{s}^{-2}$ in the upper $\approx 5 \mathrm{~m}$ during the early afternoon, and much smaller buoyancy frequencies at greater depth. At ST1, typical density and temperature profiles representative of periods IIA and IIB display large vertical stratification in the top $\approx 30 \mathrm{~m}$ at all times of the day (Fig.4.24a,b, top and bottom). The vertical variation of salinity was greatest at depths $>10 \mathrm{~m}$, but did not determine the value of $N^{2}$ above $\approx 35$-m depth to lowest order (Fig.4.24a,b, middle). Characteristic buoyancy frequencies were $O\left(10^{-4}\right) \mathrm{s}^{-2}$ in the upper $30 \mathrm{~m}$ and $O\left(10^{-3}-10^{-4}\right) \mathrm{s}^{-2}$ immediately below the surface (Figs.4.19 and 4.21). 
A simple conceptual model illustrates that the effects of night-time cooling on the density distribution at ST1 are limited. Assuming that at time $t=t_{0}$, the water column is linearly stratified between the surface and the base of the thermocline and vertically homogeneous at depth to lowest order (Fig.4.24, bottom), the density profile can be approximated by

$$
\left.\rho(z)\right|_{t_{0}}=\left\{\begin{array}{cl}
\rho_{b}-\frac{\rho_{0} N_{0}^{2}}{g}\left(z+D_{T}\right) & \text { for } \quad z>-D_{T} \\
\rho_{b} & \text { for } \quad z \leq-D_{T}
\end{array}\right.
$$

where $z=0$ at the surface and positive upward, $D_{T}$ is the vertical extent of the day-time thermocline, and $N_{0}$ is the constant buoyancy frequency in the upper layer. If the water column is cooled just enough from $t_{0}$ to $t_{1}$ that

$$
\left.\rho(z)\right|_{t_{1}}=\rho_{b} \quad \text { for all } z
$$

then the pycnocline is completely eroded by surface heat loss. Taking the onedimensional heat-balance integrated over the cooling period $\Delta t=t_{1}-t_{0}$ and assuming the surface heat loss $Q$ is a constant yields

$$
\left.\int_{-D_{T}}^{0} \rho(z) d z\right|_{t_{1}}-\left.\int_{-D_{T}}^{0} \rho(z) d z\right|_{t_{0}}=-\frac{\alpha Q}{c_{p}} \Delta t
$$

where $\alpha$ is the thermal expansion coefficient and $c_{p}$ is the specific heat at constant pressure. With (21) and (22), equation (23) may be solved for $D_{T}$ to form the expression

$$
D_{T}=\left(\frac{-2 \alpha g Q \Delta t}{c_{p} N_{0}^{2} \rho_{0}}\right)^{1 / 2} .
$$

During times of calm winds, temporal changes in the density distribution are predominantly caused by surface heat flux. For such conditions, $D_{T}$ gives an upper limit for the thickness of the thermocline that can be completely eroded by surface cooling. Taking $Q=-33 \mathrm{~W} \mathrm{~m}^{-2}\left(-41 \mathrm{~W} \mathrm{~m}^{-2}\right)$ corresponding to the average cooling rate during period IIA (IIB) when heat loss occured, $\Delta t=10 \mathrm{hrs}$ representative 
of the length of the cooling cycle, $\alpha=2000 \cdot 10^{-7} \mathrm{~K}^{-1}, c_{p}=4000 \mathrm{~J} \mathrm{~kg}^{-1} \mathrm{~K}^{-1}$, and $N_{0}^{2}=2 \cdot 10^{-4} \mathrm{~s}^{-2}\left(8 \cdot 10^{-4} \mathrm{~s}^{-2}\right)$ for period IIA (IIB) (Fig.4.21), expression (24) yields $D_{T} \approx 2.5 \mathrm{~m}(1.5 \mathrm{~m})$ in good agreement with Fig.4.23. Observations show the thermocline extended from the surface to about 30-m depth (Fig.4.24), so that the average night-time cooling was not large enough to homogenize the water column in more than the upper $\approx 10 \%$ of the stratified surface layer. Occasional cooling events of up to $-100 \mathrm{~W} \mathrm{~m}^{-2}$ may have eroded $\approx 20 \%$ of the day-time thermocline. For the PWP case, $Q=-(100-300) \mathrm{W} \mathrm{m}^{-2}$ and $N_{0}^{2} \approx 10^{-4} \mathrm{~s}^{-2}$, giving $D_{T} \approx 6-10 \mathrm{~m}$ based on (24). In comparison, the thickness of the day-time thermocline was only about $5 \mathrm{~m}$, as opposed to $30 \mathrm{~m}$ at ST1. Thus, night-time cooling alone was sufficient to erode the main thermocline in the PWP case and create a homogenous density distribution that extended to large depth. The result was strong diurnal changes in mixed layer thickness which exceeded the variations at ST1 by one order of magnitude.

\subsubsection{Low-Frequency Variability}

Spectral analysis of unfiltered, hourly-averaged estimates of surface heat flux, buoyancy frequency, wind stress, and mixed layer depth presents one way to illustrate the temporal scales on which these parameters vary and influence each other. Fig.4.25a shows about $79 \%$ of the variance in $Q$ lies in the diurnal frequency band (0.9-1.1 cpd) for periods IIA and IIB combined, a result that clearly reflects the daily heating and cooling cycle. Buoyancy frequencies $N_{S}^{2}$, where the subscript denotes the vertical average over the upper $11 \mathrm{~m}$ has been taken, display different behavior. About $55 \%$ of the variance in $N_{S}^{2}$ occurs on time scales greater than diurnal, and about $47 \%$ is contained at frequencies $<0.4 \mathrm{cpd}$ (Fig.4.25b). Approximately $87 \%$ of the wind stress variance lies within subdiurnal frequencies, and about $62 \%$ in the low frequency range $<0.4 \mathrm{cpd}$ ( $>2.5$ days) (Fig.4.25c). The observed behavior of $\tau_{W}$ and $N_{S}^{2}$ suggests low frequency variation of the wind field caused similar variation of the density distribution near the surface. This result is supported by cross spectra which indicate the coherence between $\tau_{W}$ and $N_{S}^{2}$ is 0.65-0.72 for time scales $2.5-5$ days $(0.2-0.4 \mathrm{cpd})$ and significant at the $95 \%$ confidence level $c_{95}=0.63$. The variance spectrum of the mixed layer depth shows about $42 \%$ of the variance occurs on time scales $>2.5$ days $(0.4 \mathrm{cpd})$, while less than $12 \%$ lie within the diurnal frequency band (Fig.4.25d). For temporal variation 
$>1.5$ days $(<0.6 \mathrm{cpd})$, the mixed layer depth and $\tau_{W}$ are strongly related with coherence coefficients between 0.82-0.87. Cross spectra of mixed layer depth and $N_{S}^{2}$ give the coherence coefficients $0.63-0.66$ for time scales $2.5-5$ days $(0.2-0.4)$ cpd. These results together with the observed coherence between $\tau_{W}$ and $N_{S}^{2}$ indicate low-frequency variability in wind stress induced changes in vertical stratification and mixed layer thickness on similar time scales. Cross spectra between $h_{m}$ and $Q$ display no coherence at frequencies outside the diurnal band. However, it should be noted that seasonal changes cannot be resolved given the length of period II and the band-width averaging applied for analysis.

The observed variability of vertical stratification, wind stress, and mixed layer depth on periods of days implies that the wind-induced circulation varies on similar time scales. Rigorous investigation of the question how low-frequency variability affects the ensemble-averaged currents requires an event analysis be performed corresponding to the one carried out for the winter period. The results from such analysis would aid in the understanding of the instantaneous response to individual wind events, and how these events combine to form the ensemble-averaged velocity distribution. Event analysis was attempted for several subintervals of period IIA and IIB, but without great success. Although a few time periods of 1-2-days length could be identified where the agreement between the observed transport magnitudes and $\frac{\bar{\tau}_{W}}{\rho_{0} f}$ was reasonable (e.g., May 29-30, June 27-29), the transport vector was characteristically $60-75^{\circ}$ to the right of the wind rather than $90^{\circ}$ as predicted by theory (not shown). This behavior may be due to wave bias, since the events chosen for analysis were marked by strong winds $\left(\frac{\bar{\tau}_{W}}{\rho_{0}} \geq 1 \mathrm{~cm}^{2} \mathrm{~s}^{-2}\right)$ compared to typical, less energetic summer conditions. With the effects of wind forcing being largely limited to the upper $14 \mathrm{~m}$ or less during period II, disagreement of $15-30^{\circ}$ imposes a comparable uncertainty on the direction of the velocity estimates everywhere in the wind-induced shear layer. Hence, no reliable information could be gained about the relative direction of the current vectors, their rotation with depth, and the flatness of the observed spirals during individual wind events.

Despite the fact that conclusions concerning the immediate response to wind forcing cannot be made, the ensemble averaged results give some indication about the nature of the wind-driven flow during stratified conditions. In particular, the nearly unidirectional nature of the wind-induced flow toward the right of the wind can be related to buoyancy forcing. This can be demonstrated by considering the 
simplistic scenario of a wind mixed layer above a main thermocline, where the vertical extent of the wind-driven current shear is limited by the mixed layer thickness. Defining $D_{S}$ as the depth of semi-permanent stratification (i.e., the top of the seasonal thermocline), and applying the boundary conditions $K \frac{\partial \mathbf{V}_{W}}{\partial z}=\frac{\boldsymbol{\tau}_{W}}{\rho_{0}}$ at $z=0$ and $K \frac{\partial \mathbf{V}_{W}}{\partial z}=0$ at $z=-D_{S}$ gives under the assumption that $K=$ constant (Levitus, 1982; PS)

$$
\mathbf{V}_{W}=U_{H} \alpha \frac{r}{1-s}\left[\exp \left(r \alpha z^{\prime}\right)+s \exp \left(-r \alpha z^{\prime}\right)\right]
$$

In (25), $z^{\prime}=z / D_{S}, U_{H}=\tau_{W} /\left(\rho_{0} D_{S}\right), \alpha=D_{S} / D_{E} \quad\left(D_{E}=\left(\frac{2 K}{f}\right)^{1 / 2}\right.$ is the Ekman scale depth from (15)), $r=\sqrt{2} \exp \left(\frac{i \pi}{4}\right)$, and $s=\exp (-2 r \alpha)$. With $D_{S}=h_{m}$, where $h_{m}$ is the mixed layer depth defined in section 4.2.7, and substituting the diffusive length scale $\ell_{W}=\frac{\kappa u_{* W}}{f}$ as an approximation for the unstratified Ekman layer depth $D_{E}$, the degree of surface-trapping based on least squares fits of daily averaged estimates $h_{m}$ to $D_{E}$ is $\alpha=0.12 \pm 0.07$ for period IIA and $\alpha=$ $0.06 \pm 0.04$ for period IIB (Fig.4.26). Values denoted by \pm give the $95 \%$ uncertainty estimates of the fits and have been computed assuming one independent estimate every two days. The intersect of the slope with the $y$-axis was taken to be zero. Based on the $\alpha$ estimates given above, the region where turbulent momentum acts to homogenize the water column is about one order of magnitude smaller than the diffusive depth scale during unstratified conditions. Expansion to fourth order in $\alpha$ shows for small $\alpha$ expression (25) simplifies to (PS)

$$
\mathbf{V}_{W}=U_{H}\left[1+i \alpha^{2}\left(z^{2}+2 z^{\prime}+2 / 3\right)\right]
$$

According to (26), the flow field's response to wind forcing becomes increasingly slablike in the cross-wind direction as $\alpha$ approaches zero. The physical explanation is that the time it takes for the turbulent momentum to diffuse to the base of the mixed layer is much smaller than the rotational time scale $\frac{1}{f}$. This can be shown from simple scaling: In the absence of rotation, the momentum balance scales according to

$$
\frac{\Delta U}{\Delta T_{K}} \sim K \frac{\Delta U}{D_{S}^{2}}
$$


where it has been assumed that vertical diffusion is limited to the region above the thermocline. Expression (27) can be solved to give the diffusive time scale

$$
\begin{aligned}
\Delta T_{K} & \sim\left(\frac{D_{S}}{D_{E}}\right)^{2} \frac{2}{f} \\
& \sim 2 \alpha^{2} T_{f}
\end{aligned}
$$

where $T_{f}=\frac{1}{f}$ and $K=\frac{D_{E}^{2} f}{2}$ have been substituted. Thus, $T_{K}<<T_{f}$ for small $\alpha$, and vertical diffusion is hardly affected by rotation. As a result, the windinduced currents display little veering with depth, and in order to satisfy the zero stress condition at the top of the thermocline form a slab directed to the right of the wind.

Expression (26) predicts the crosswind flow between $z=0$ and $z=-D_{S}$ is vertically uniform, while the downwind flow is sheared but extremely small $\left(O\left(\alpha^{2}\right)\right)$. In contrast, measurements representative of period II suggest the crosswind shear is at least one order of magnitude larger than the downwind shear, with the possible exception of the currents in the upper $\approx 7.5 \mathrm{~m}$ during period IIA (Fig.4.20a). The discrepancy between (26) and the observations may have several reasons. First, it is obvious that (26) represents a highly idealized solution for the wind-induced circulation and is only partly applicable to the flow conditions at ST1. Derivation of (26) a priori limits vertical diffusion to the extent of the mixed layer, i.e., the region where $K$ is assumed to be constant. In a more realistic scenario, the turbulent momentum diffuses into the stratified interior until it reaches a depth where the criterion for linear instability, $R i<0.25$, is no longer met. This statement is consistent with results from section 4.3.1 indicating that the mixed layer depth is shallower than the vertical extent of the region in which mixing occurs (see also Fig.4.11). Within the stratified regime, the inhibiting effects of buoyancy forcing cause diffusion to take place on time scales that are larger than in the homogeneous surface layer. As a result, the wind-induced currents below the base of the mixed layer are affected by the Coriolis force and turn to the right with increasing depth. The turning rate of the resulting current spiral depends on the details of the mixing processes and cannot be modeled without application of an appropriate turbulence closure scheme. Meaningful parameterization of turbulent mixing in the stratified 
surface layer has been subject to several studies (e.g., PWP, PS) and remains a topic of open discussion.

An alternative explanation for the observed crosswind shear is that the immediate response to wind forcing is slablike, and that the average over several slabs determines the vertical structure of the ensemble-averaged flow. The depth of the wind-induced slab layer depends on the magnitude of the wind stress and varies from event to event. Only the strongest events can cause flow at large depths, while weak as well a strong events drive the near-surface currents. Hence, taking the temporal mean over times of weak and strong winds introduces a bias toward the current response at small depths and gives a decreasing velocity distribution with increasing distance from the surface. The validity of this hypothesis cannot be tested without the ability to provide reliable estimates of the flow conditions during individual wind events. As discussed above, the estimation method applied here is not precise enough to allow for investigation of the wind-driven flow on temporal scales of 1-2 days comparable to the width of the intermittent, narrow peaks in wind stress magnitude representative of summer (Fig.4.19a). However, bias associated with the averaging procedure cannot be ruled out, particularly since results from the coherence analysis illustrate the mixed layer depth is a strong function of wind stress. Low pass-filtered values of $h_{m}$ cover the range $0-10 \mathrm{~m}(0-5 \mathrm{~m})$ for period IIA (IIB), with the largest values occuring at times of strongest winds (Fig.4.27). Since large winds take up a significantly lesser fraction of period II than weak to moderate winds (e.g., $\frac{\tau_{w}}{\rho_{0}}>1 \mathrm{~cm} \mathrm{~s}^{-2}$ for less than $11 \%$ and $3 \%$ of period IIA and IIB, respectively), it is to be expected the ensemble-averaged results are biased toward the wind-induced currents during times of small wind stress and mixed layer depth.

\subsection{Summary and Conclusions}

- From the measurements, all terms in the subtidal momentum balance with exception of the surface pressure gradient could be estimated. Closing the momentum balance by solving for the sea surface slope indicates the time-mean, depth-averaged subtidal dynamics are largely described by a balance between the Coriolis terms and pressure forcing. Results suggest about $45 \%$ (85\%) of the along-bank flow aver- 
aged over periods of 2-3 months were in geostrophic equilibrium with the combined barotropic and baroclinic cross-bank pressure gradient in winter (summer). In summer, increasing lateral stratification caused a depth-averaged baroclinic pressure gradient that opposed the mean on-bank slope of the sea surface. The result was larger along-bank transport in summer than in winter, in agreement with previous observations by Butman and Beardsley (1987).

Data suggest that the depth-averaged cross-bank flow was off-bank in the timemean, with transport estimates that were about $40 \%$ and $25 \%$ of the along-bank transport for winter and summer, respectively. Closing the subtidal momentum balance indicates the off-bank flow may have been in near-balance with the sea surface slope, yielding increasing sea level elevations toward the southwest. This result opposes the idea upstream forcing causes an along-shelf pressure gradient in order to balance bottom friction as suggested by Beardsley and Hart (1978), Beardsley and Winant (1979), and Chapman et al. (1986). The inference is local deviations from the predominantly along-bank orientation of the isobaths may cause cross-bank transport and along-bank pressure forcing on spatial scales smaller than those of the large-scale circulation.

- On time scales smaller than the long-term mean, the surface wind stress reached magnitudes comparable to those of the Coriolis and pressure terms. This was particularly obvious in winter when temporal variation of the wind forcing was large and occured on time scales of days. In the analysis of the subtidal momentum balance, temporal changes in wind forcing manifest themselves in the form of large standard deviations from the relatively small time-mean cross- and along-bank wind stress components.

Previous analysis of long-term moored array data as well as numerical studies have shown that variation in along-bank wind stress is strongly related to variation in along-bank flow, with the currents lagging the wind by $\approx 9 \mathrm{hrs}$ (Noble et al., 1985, Greenberg et al., 1997). Measurements from ST1 support these conclusions, but indicate the current response occured on shorter time scales ( $\approx 3 \mathrm{hrs}$ ) in summer than in winter. The observed variation in phase-lag is in qualitative agreement with observations by Brown (1985), who found bottom pressure gradients off Nantucket Shoals respond faster to along-bank wind forcing in summer than in winter.

Data also indicate that the correlation between the along-bank wind stress and 
cross-bank transport is statistically significant. This behavior is consistent with the idea of a wind-induced flow response at right angle to the wind vector as suggested by Ekman theory.

- Bottom stress estimates are about one order of magnitude smaller than the estimated wind stress for times when the wind forcing was strong, i.e., excluding periods when both the bottom and wind stress were equally weak. This behavior illustrates that only a small part of the turbulent momentum introduced at the surface penetrated to the bottom, while most of the wind-induced momentum flux was balanced by the Coriolis force. Using the diffusive depth-scale $l_{W}=\frac{\kappa u_{*} W}{f}$ to estimate the thickness of the region affected by wind friction (Madsen, 1977) gives $l_{W}=80-90 \mathrm{~m}$ for the strongest wind events, and $l_{W}=40 \mathrm{~m}$ averaged over the energetic wind period February to mid-May. These values are comparable to the water depth at ST1 $(76 \mathrm{~m})$, supporting the conclusion that the surface wind stress was mostly balanced by Ekman transport.

- The estimated diffusion depth $l_{W}$ explains why Georges Bank is not an up/ downwelling region in the typical sense. Brink (1983) showed analytically that for an along-bank wind stress, up/downwelling occurs along the flanks of a submarine bank if $l_{W}>D_{0}$, where $D_{0}$ is the water-depth on the shallow bank plateau. Physically, the wind-induced Ekman transport cannot fully develop if $l_{W}>D_{0}$. Instead, part of the wind stress is compensated by bottom friction, and the resulting bottom Ekman layer produces a cross-bank transport that opposes the depth-integrated windinduced flow. For Georges Bank, $l_{W}>D_{0}\left(D_{0} \approx 50 \mathrm{~m}\right)$ describes about $16 \%$ of the entire data record Feb-August, 1995 (23\% if only the energetic wind period February to mid-May is considered). Hence, intermittent periods of up/downwelling may occur in regions where $D<l_{W}$, but are not an inherent feature of the Georges Bank circulation.

- The analysis of the wind-induced currents clearly demonstrates that the vertical distribution of the wind-driven circulation varies with season. Results representative of winter form a well developed spiral that extends to great depth, while results representative of summer are surface-trapped and display relatively little veering with increasing distance from the surface. Summarizing the winter and summer 
results, the conclusions are:

\section{Winter:}

- The spiral structure of the winter currents is in agreement with earlier observations by Schudlich and Price 1998) for the deep ocean. However, Schudlich and Price (1998) suspected their measurements were subject to strong wave-bias, which greatly increased the downwind shear in the upper $\approx 15 \mathrm{~m}$ and caused a depth-integrated transport vector that was $70^{\circ}$ rather than $90^{\circ}$ to the right of the wind direction. In the present case, observations suggest wave-bias was limited to the upper $\approx 5 \mathrm{~m}$. This follows from the estimated downwind shear of the wind-driven currents, which is about twice as large between 5-7.5-m than at greater depth.

- The vertical structure of the wind-induced currents in winter is qualitatively similar to the classical Ekman spiral. For the long-term average as well as for individual wind events, the velocity vector rotates clockwise with depth, at the same time decreasing in magnitude. Based on the ensemble averaged results, a characteristic scale depth describing the decay of the current magnitude away from the sea surface is $L_{e} \approx 12 \mathrm{~m}$, compared to the larger 1-radian rotation depth of the velocity vector $L_{\theta} \approx 22 \mathrm{~m}\left(\frac{L_{\theta}}{L_{e}} \approx 1.8\right)$. In comparison, $L_{e} \approx 12-18 \mathrm{~m}$ for individual wind events with time scales 1.2-2.6 days. Results from the event analysis show $L_{e}$ approximately matches the 1-radian rotation depth of the velocity vector $\left(L_{\theta}=14-19\right)$, in agreement with Ekman's (1905) analytical solution for wind-driven flow. On the other hand, the observed current amplitudes are significantly smaller than those predicted by Ekman theory, indicating the assumption of depth-independent $K$ cannot explain the observations.

- All three wind events examined here occured in conjunction with strong surface cooling, giving rise to the speculation that convection acted to increase and homogenize the eddy viscosity throughout large parts of the water column. The implication is that $K$ was not as strong a function of depth as in the case of purely mechanical turbulence, where the size of the turbulent eddies is limited by their distance from the nearest (surface or bottom) 
boundary. However, the observed underestimation of the wind-driven currents by Ekman theory indicates that the eddy viscosity was not completely depth-independent.

The ensemble average includes times of surface heating as well as cooling, so that the time-mean $K$-distribution may display more variation with depth than the corresponding distribution during isolated periods of strong surface heat loss. It is possible that large and relatively uniform $K$ accounts for the observed flatness $F=\frac{L_{\theta}}{L_{e}} \approx 1$ that is characteristic to all three wind and cooling events described here. The flatter structure of the ensembleaveraged currents $(F \approx 1.8)$ may be a result of averaging over periods with predominantly mechanical and convective mixing.

- Although based on in-situ observations, the inferences about the $K$ distribution drawn above are strictly speculative. Further investigation of this question requires direct measurements of the turbulence quantities be made, and the mixing parameters and current distributions be compared for times with and without surface cooling. It is worthwhile noting that analysis of the winter currents in the absence of surface cooling was attempted, i.e., for wind events with temporal scales of hours to days, but at times when $Q$ was weak and positive. The results of this analysis were not useful, mainly because no strong, well defined wind events could be identified that fulfilled the requirement $Q>0$.

- Comparison of the ensemble-averaged results to observations from the winter event analysis illustrates that the flow response to wind forcing can be significantly more energetic on time scales of days than in the long-term mean. The inference is implementation of the monthly or bimonthly averaged wind stress vector in three-dimensional numerical models may not be sufficient to explain physical and biological processes on Georges Bank.

\section{Summer:}

- The ensemble-averaged results for summer indicate the wind-induced currents are surface-trapped at depths $<10-19 \mathrm{~m}$. Reasons for the surfacetrapping include strong vertical stratification as well as generally weak winds. 
According to the long-term average, the wind-driven circulation is almost entirely in the cross-wind direction, with velocity amplitudes that decay rapidly with depth. This result is partly supported by earlier observations representative of the deep ocean (e.g., Price et al., 1987), which showed the clockwise rotation of the velocity vector is small during stratified conditions and gives the spiral an overall "flat" $\left(\frac{L_{\theta}}{L_{e}}>1\right)$ appearance.

Price et al. (1987) argued the observed flatness is a consequence of diurnal cycling, which limits the current response to a thin surface layer during the daylight hours, while it extends to greater depths at night. In the present case, diurnal cycling does not affect the wind-induced currents to a degree that can be detected by the measurements. This follows from the observations that a) the ensemble-averaged day- and night-time currents are almost identical at ST1, and b) the main thermocline covers a greater depth-range than in Price et al.'s (1986) case ( $30 \mathrm{~m}$ as opposed to $5 \mathrm{~m}$ ), and cannot be eroded by occasional night-time cooling. Observations reveal typical variations in mixed layer depth are about 1.5-2.5 m, indicating the effects of diurnal cycling on the velocity distribution are not resolved by the VMCMs.

- Spectral analysis suggests variation in wind stress on time scales of days causes variation of the near-surface stratification and mixed layer depth that happens on similar time scales. The impact of such low-frequency variability on the ensemble-averaged wind-driven circulation is unclear, but may be explained as follows: during times of strong winds, the wind-induced currents extend to larger depth than during times when the wind is moderate or weak. Assuming that each wind event enforces a more or less slablike response in the surface mixed layer followed by a sheared regime immediately below the mixed layer base, the long-term average over several such slabs would be biased toward the near-surface region where the effects of wind forcing are felt most frequently (i.e., during periods of weak and strong winds). The resulting long-term average may give a velocity profile that is to the right of the wind and decreases with depth, very much as suggested by the observations.

- More detailed conclusions about the current response to wind forcing during stratified conditions cannot be drawn, unless the wind-induced currents are investigated on time scales corresponding to those of individual wind events. 
Such analysis was attempted but did not give additional insight, since meaningful interpretation required the results were accurate to a degree not provided by the measurements or the estimation method. This realization is not surprising, considering that even the strongest wind events (and therefore the wind-induced currents) are significantly less energetic than the winter storms for which an event analysis was possible. Hence, the investigation of the summer period had to be limited to the long-term average, which gave more robust estimates than could be obtained for short time scales.

- The results presented in this chapter give an extensive description of the subtidal dynamics on the southern flank of Georges Bank, particularly concerning the response of the flow. field to wind forcing. For the first time, estimates of the ageostrophic wind-driven currents, i.e., the component of the wind-induced flow that is not balanced by local pressure gradients, were made for the southern flank of Georges Bank. The results of this analysis clearly indicate that the wind-driven circulation cannot be completely understood by investigation of the long-term average alone. In addition, time scales comparable to those of individual wind events have to be examined, and the physical processes determining the vertical structure of the wind-induced flow have to be identified for various background conditions (e.g., periods of surface heating and cooling, homogeneous and stratified conditions). 


\section{References}

Beardsley. R.C., and J. Hart, A simple theoretical model for the flow of an estuary onto a continental shelf. J. Geophys. Res.,83, 873-883, 1978.

Beardsley. R.C., and C. D. Winant, On the mean circulation in the Mid-Atlantic Bight. J. Phys. Oceanogr., 9, 612-219, 1979.

Brink, K.H., Low-frequency free wave and wind-driven ,options over a submarine bank. J. Phys. Oceanogr., 13, 103-116, 1983.

Brown, W.S., N.R. Pettigrew, and J.D. Irish. The Nantucket Shoals Flux Experiment (NSFE79). Part II: The structure and variability of across-shelf pressure gradients. J. Phys. Oceanogr, 15, 749-771, 1985.

Brown, W. S., the wind-forced pressure response of the Gulf of Maine. Submitted to J. Geophys. Res..

Butman, B., M. Noble, D. C. Chapman and R. C. Beardsley, An upper bound for the tidally rectified current at one location on the southern flank of Georges Bank. J. Physical Oceanogr., 11, 1452-1460, 1983.

Butman, B., and R.C. Beardsley, Long-term observations on the southern flank of Georges Bank. Part I: A description of the seasonal cycle of currents, temperature, stratification, and wind stress. J. Phys. Oceanogr., 17, 367-384, 1987.

Chapman, D. C. and R. C. Beardsley, On the origin of shelf water in the Middle Atlantic Bight. J. Physical Oceanogr., 19, 384-390, 1989.

Chapman, D., J. Barth, R.C. Beardsley, and R. Fairbanks, On the continuity of mean flow between the Scotia Shelf and the Middle Atlantic Bight. J. Phys. Oceanogr., 16, 758-772, 1986.

Chereskin, T.K., Direct evidence for an Ekman balance in the California Current. J. Geophys. Res.,100, 869-878, 1985.

Chen, C., Variability of currents in Great South Channel and over Georges Bank: Observation and Modeling. Ph.D. Thesis, Joint Program in Oceanography/ 
Applied Ocean Science and Engineering, Woods Hole Oceanographic Institution, Woods Hole, Mass, Massachusetts Institute of Technology, Cambridge, Mass, 1992.

Ekman, V. W., On the influence of the earth's rotation on ocean-currents. Ark. Mat., Astron. \& Fys., 2, 55, 1905.

Grant, W. D., and O. S. Madsen, Combined wave and current interaction with a rough bottom. J. Geophys. Res., 84, 1,797-1,808, 1979.

Greenberg, D.A., J.W. Loder, Y. Shen, D. R. Lynch, and C.E. Naimie, Spatial and temporal structure of the barotropic response of the Scotian Shelf and Gulf of Maine to surface wind stress: A model-based study. J. Geophys. Res., 102, 20,897-20,915, 1997.

Kitaigorodskii, S.A., M.A. Donelan, J.L. Lumley, and E.A. Terray, Wave-turbulence interactions in the upper ocean. Part II: Statistical characteristics of wave and turbulent components of the random velocity field in the marine surface layer. J. Phys. Oceanogr., 13, 1988-1999, 1983.

Large, W. G., and S. Pond, Open ocean flux measurements in moderate to strong winds, J. Physical Oceanogr., 11, 324-336, 1981.

Lentz, S.L., The surface boundary layer in coastal upwelling regions. J. Phys. Oceanogr., 22, 1517-1539, 1992.

Levitus, S, Climatological atlas of the world ocean, NOAA professional paper 13, US. Dept. of Commerce, NOAA, 1982.

Mellor, G. L., and T. Yamada, Development of a turbulence closure model for geophysical fluid problems. Rev. Geophys. Space Physics, 20, 851-875, 1982.

Loder, J. W., Topographic rectification of tidal currents on the sides of Georges Bank. J. Physical Oceanogr., 10, 1399-1416, 1980.

Lynch, D. R., F. E. Werner, D. A. Greenberg, and J. W. Loder, Diagnostic model for baroclinic, wind-driven and tidal circulation in shallow seas. Continental Shelf Res., 12, 37-64, 1992. 
Lynch, D.R., J.T.C. Ip, C.E. Naimie, and F.E. Werner, Comprehensive coastal circulation model with application to the Gulf of Maine. Cont. Shelf Res., 16, 875-906, 1996.

Madsen, O.S., A realistic model of the wind-induced Ekman boundary layer. $J$. Phys. Oceanogr., 7, 248-255, 1977.

Naimie, C. E., Georges Bank residual circulation during weak and strong stratification periods: Prognostic numerical model results. J. Geophys. Res.,101, 6469-6486, 1996.

Noble, M., and B. Butman, and M. Wimbush, Wind-current coupling on the southern flank of Georges Bank: Variation with season and frequency. J. Phys. Oceanogr., 15, 604-620, 1985.

Pollard, J. F., P.B. Rhines, and R.O.R.Y. Thompson, The deepening of the windmixed layer. Geophys. Fluid Dyn,,3, 381-404, 1973.

Price, J.F., R.A. Weller, and R. Pinkel, Diurnal cycling: Observations and models of the upper ocean response to diurnal heating, cooling, and wind mixing. $J$. Geophys. Res.,91, 8411-8427, 1986.

Price, J.F, R.A. Weller, and R.S. Schudlich, Wind-driven ocean currents and Ekman transport. Science, 238, 1534-1538, 1987.

Price, J.F., and M.A. Sundermeyer, Stratified Ekman Layers. Submitted to $J$. Geophys. Res..

Santala, M.J., Surface-referenced current meter measurements. Ph.D. Thesis, Joint Program in Oceanography/ Applied Ocean Science and Engineering, Woods Hole Oceanographic Institution, Woods Hole, Mass, Massachusetts Institute of Technology, Cambridge, Mass, 1991.

Schudlich, R.R., and James F. Price, Observations of Seasonal Variation in the Ekman Layer. J. Phys. Oceanogr., 28 , 1187-1204, 1998.

Winant, C. D., and R. C. Beardsley, A comparison of some shallow wind-driven currents. J. Phys. Oceanogr., 8 , 218-220, 1979. 
Cross-Bank $(+\mathrm{x})$

\begin{tabular}{l|c|c|c|c|c|c|c}
$\cdot 10^{-6} \mathrm{~m} \mathrm{~s}^{-2}$ & $\frac{\partial[u]}{\partial t}$ & {$\left[\frac{\partial\left(u^{2}\right)}{\partial x}\right]$} & $f[v]$ & $g \frac{\partial \eta}{\partial x}$ & $\frac{g}{\rho_{0}}\left[\frac{\partial \rho}{\partial x}(D+z)\right]$ & $\frac{\tau_{u}^{\tau}}{\rho_{0} D}$ & $\frac{\tau_{b}^{x}}{\rho_{0} D}$ \\
\hline Period I & $0.0 \pm 0.5$ & $1.9 \pm 0.6$ & $4.7 \pm 6.0$ & $1.1 \pm 6.7$ & $1.1 \pm 1.4$ & $-0.6 \pm 1.4$ & $-0.2 \pm 0.2$ \\
\hline Period II & $0.0 \pm 0.4$ & $1.2 \pm 0.9$ & $7.2 \pm 3.3$ & $10.7 \pm 3.9$ & $-4.6 \pm 2.2$ & $0.0 \pm 0.3$ & $0.0 \pm 0.2$
\end{tabular}

Along-Bank $(+y)$

\begin{tabular}{l|c|c|c|c|c|c|c} 
Along-Bank $(+\mathrm{y})$ \\
$\cdot 10^{-6} \mathrm{~m} \mathrm{~s}^{-2}$ & $\frac{\partial[v]}{\partial t}$ & {$\left[\frac{\partial(u v)}{\partial x}\right]$} & $f[u]$ & $g \frac{\partial \eta}{\partial y}$ & $\frac{g}{\rho_{0}}\left[\frac{\partial \rho}{\partial y}(D+z)\right]$ & $\frac{\tau_{w}^{y}}{\rho_{0} D}$ & $\frac{\tau_{b}^{y}}{\rho_{0} D}$ \\
\hline Period I & $0.0 \pm 0.9$ & $0.0 \pm 0.3$ & $-1.9 \pm 2.3$ & $1.4 \pm 1.8$ & $0.0 \pm 0.7$ & $0.0 \pm 1.5$ & $0.3 \pm 0.4$ \\
\hline Period II & $0.0 \pm 0.5$ & $0.0 \pm 0.6$ & $-1.8 \pm 1.8$ & $1.5 \pm 1.9$ & $0.0 \pm 0.9$ & $-0.2 \pm 0.5$ & $0.2 \pm 0.2$
\end{tabular}

Table 4.1: Temporal means and $( \pm)$ standard deviations of all terms in the depth-averaged subtidal (time scales $>33 \mathrm{hrs}$ ) momentum equations (7) for periods I and II. The surface pressure gradient was obtained by solving (7) for $g \nabla \eta$. At times when ST2 or SF data were not available, the baroclinic pressure forcing was set to zero for the purpose of computing the sea surface slope. 


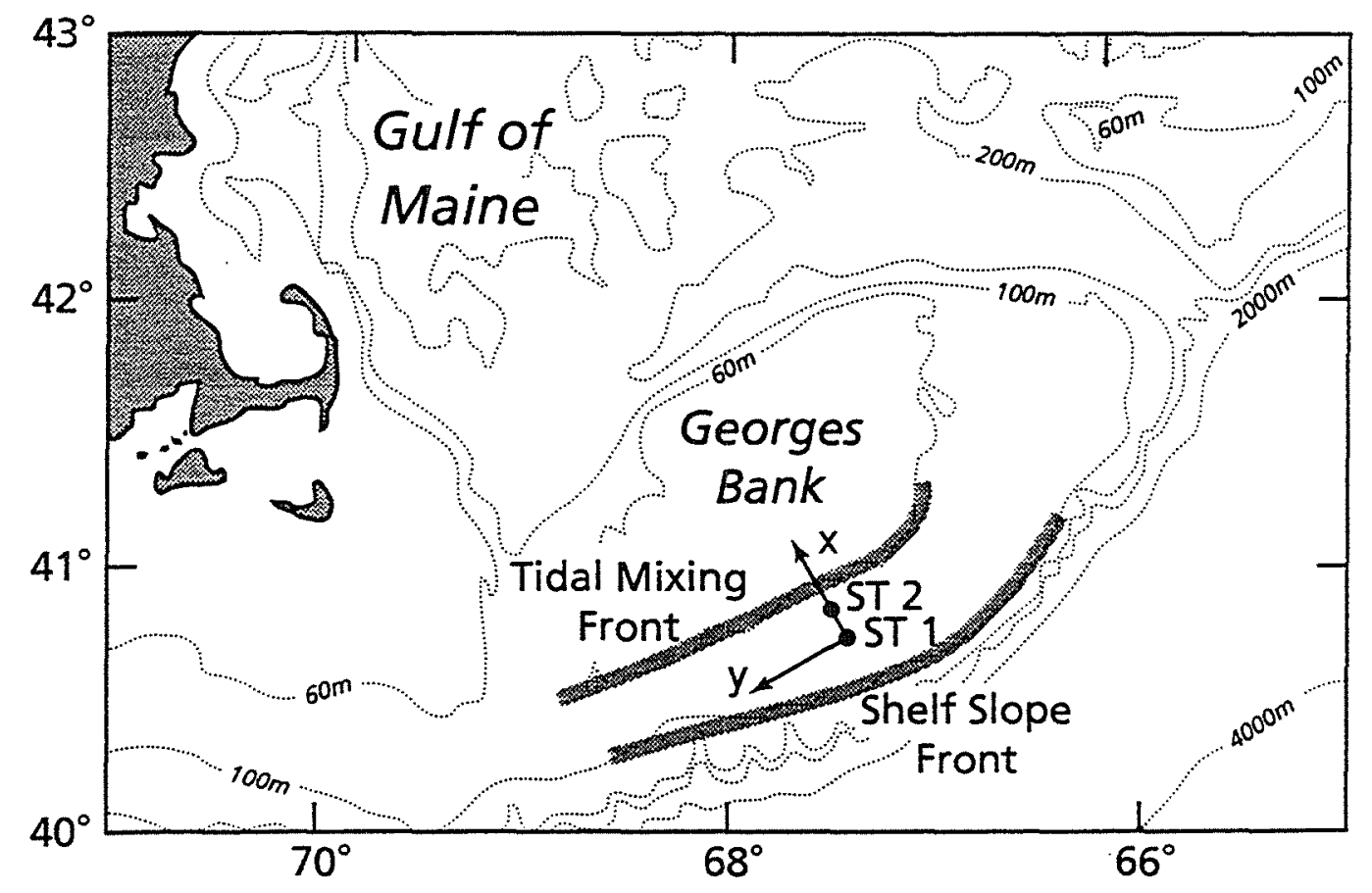

Fig.4.1: Bathymetry (in meters) of Georges Bank and adjacent region, approximate location of the Tidal Mixing Front (TMF) and Shelf-Slope Front (SSF), and the GLOBEC Stratification Study mooring sites ST1, ST2 and SF. The $+x$ direction is on-bank $\left(330^{\circ} \mathrm{T}\right)$. 


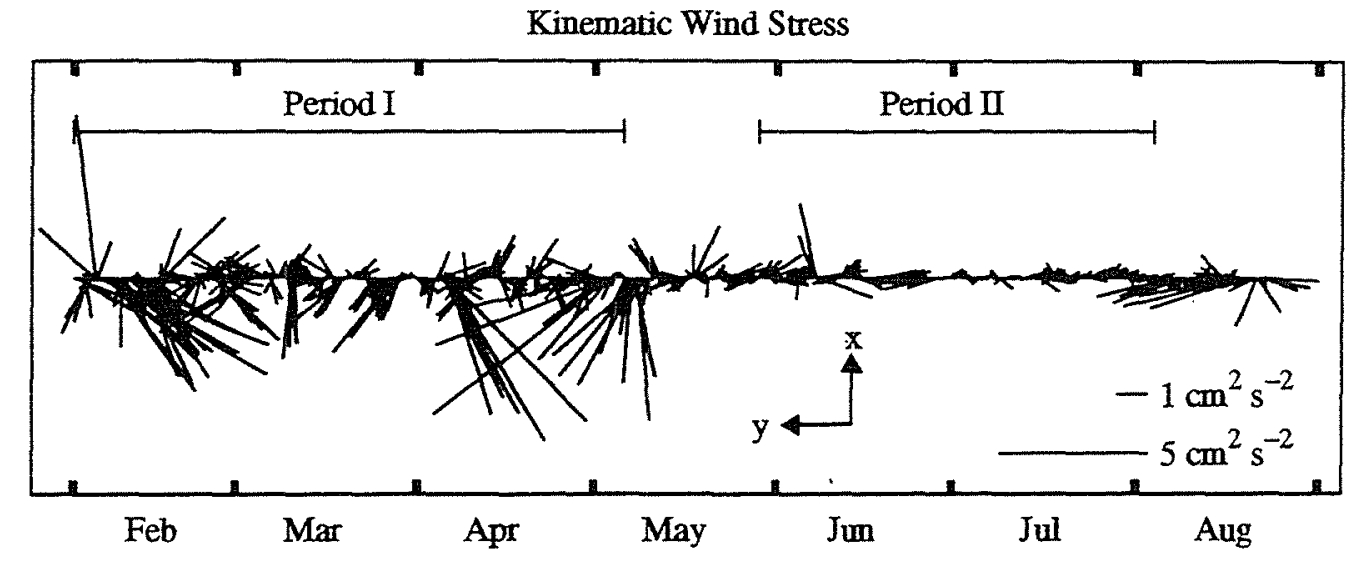

Fig.4.2: Vector-diagram of the kinematic wind stress at ST1. Each vector represents a six-hour average.

Fig.4.3: Time series of low-pass-filtered buoyancy frequency squared from hourly averaged SeaCAT data at mid depths between (solid) 6-m and 11-m depth, and (dashed) 46.5-m and 65-m depth.

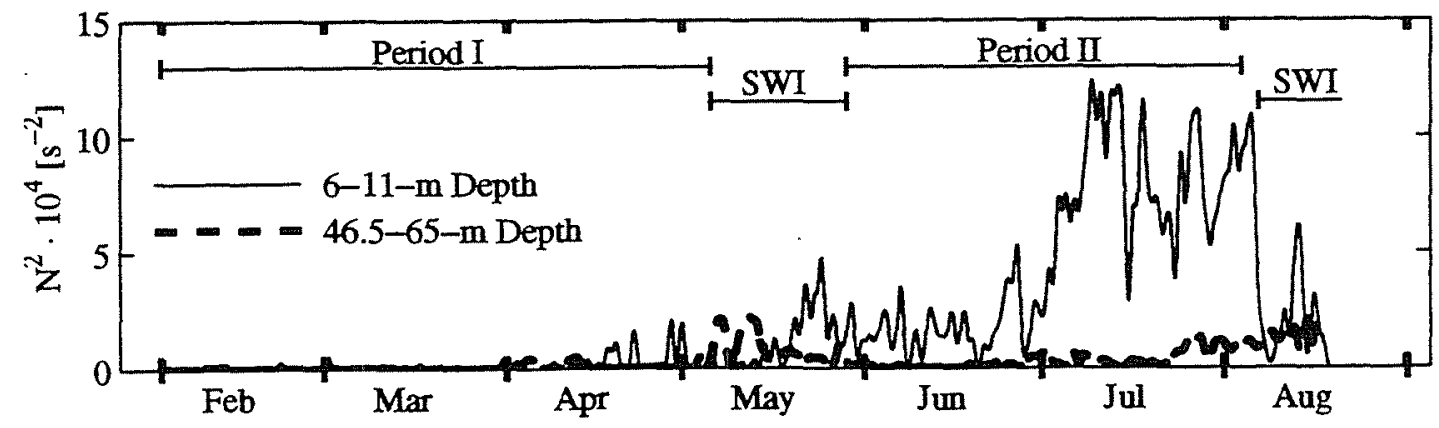


(a)
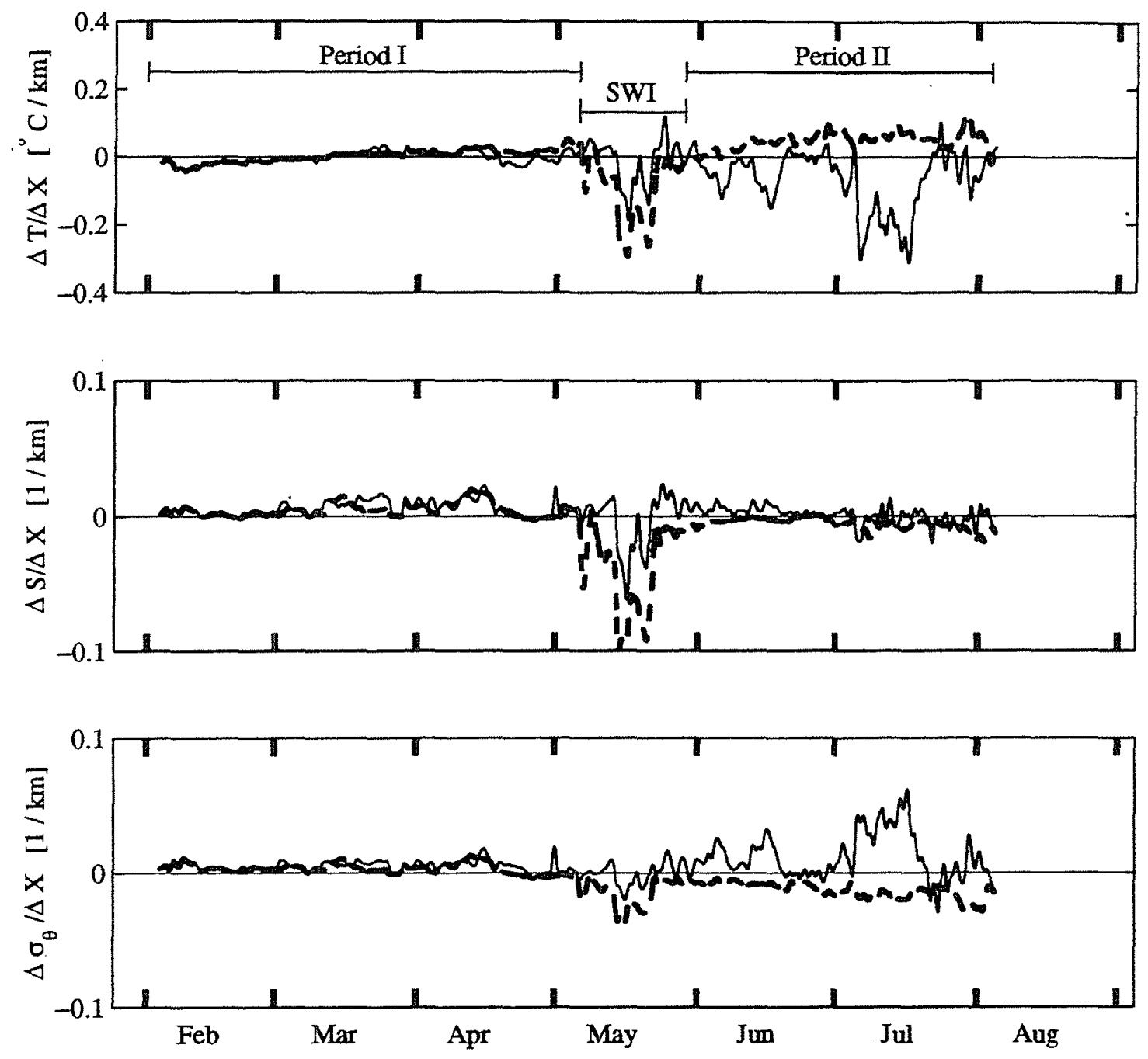

Fig.4.4a: Low-pass-filtered cross-bank gradients of (top) temperature, (middle) salinity, and (bottom) potential density gradients computed from hourly averaged ST1, ST2, and SF data. Solid lines correspond to the depth-averaged gradients in the upper $18.5 \mathrm{~m}$, and dashed lines are the depth-averages in the lower $57.5 \mathrm{~m}$. 
(b)
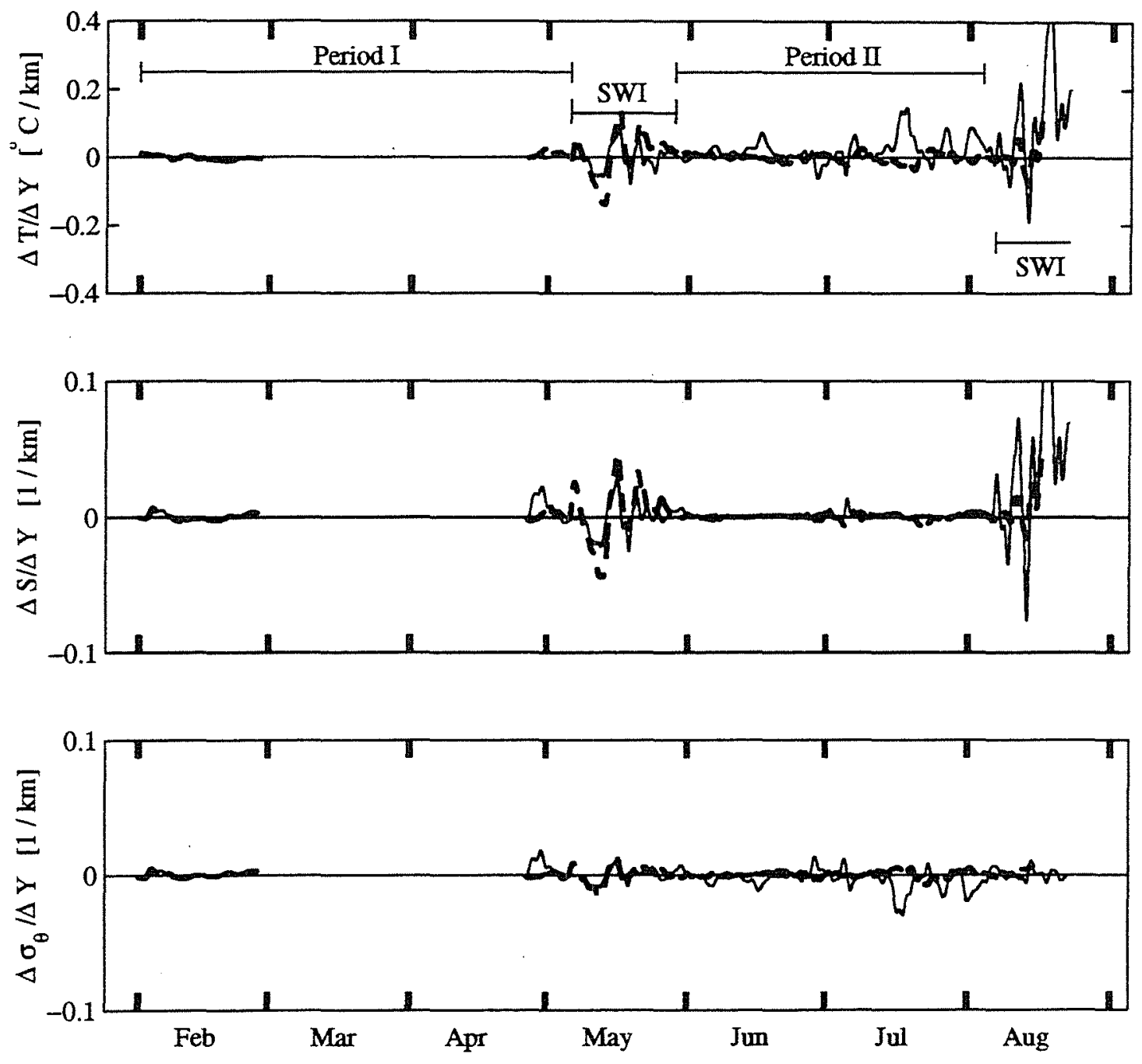

Fig.4.4b: Low-pass-filtered along-bank gradients of (top) temperature, (middle) salinity, and (bottom) potential density gradients computed from hourly averaged ST1, ST2, and SF data. Solid lines correspond to the depth-averaged gradients in the upper $18.5 \mathrm{~m}$, and dashed lines are the depth-averages in the lower $57.5 \mathrm{~m}$. 

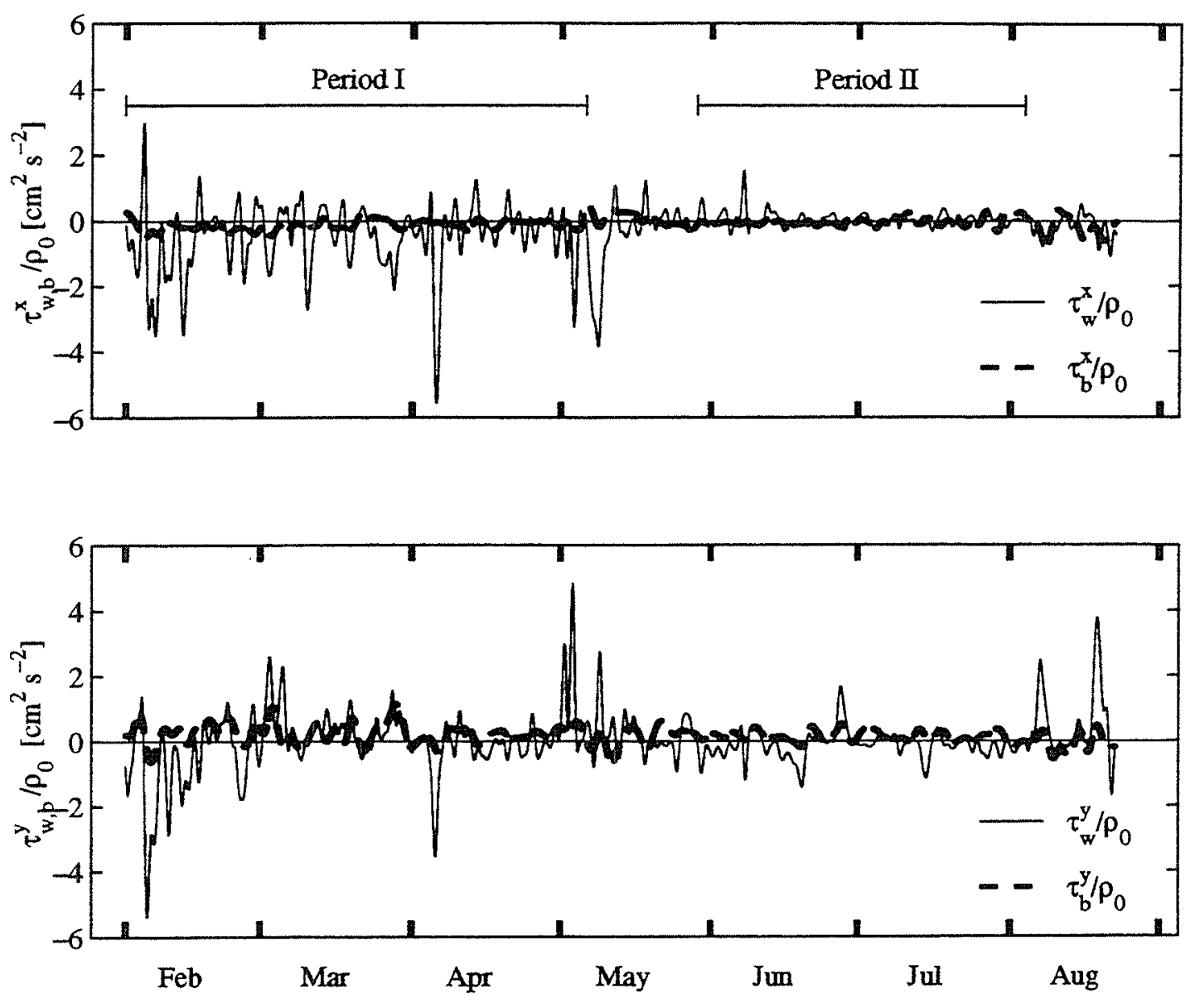

Fig.4.5: Hourly values of low-pass-filtered (solid) wind stress and (dashed) bottom stress in the (top) cross-bank and (bottom) along-bank direction. 


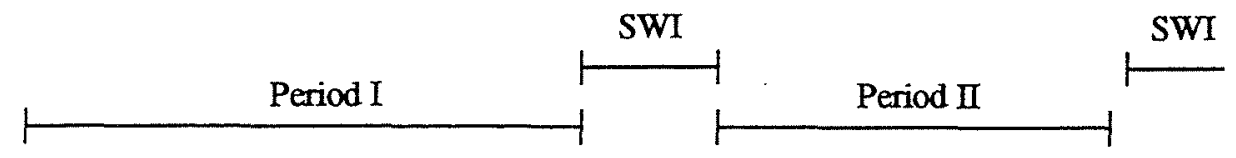

(a)

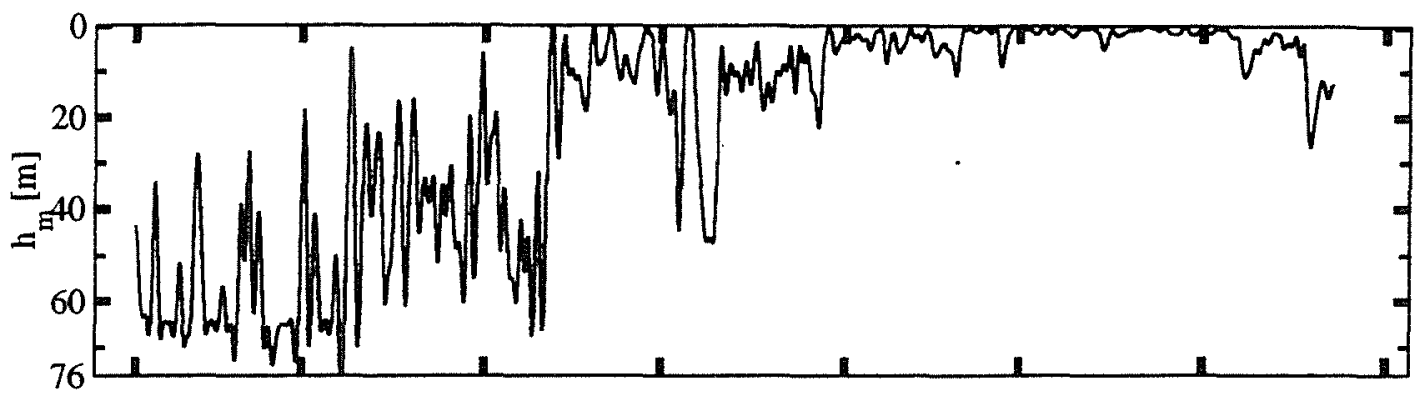

(b)

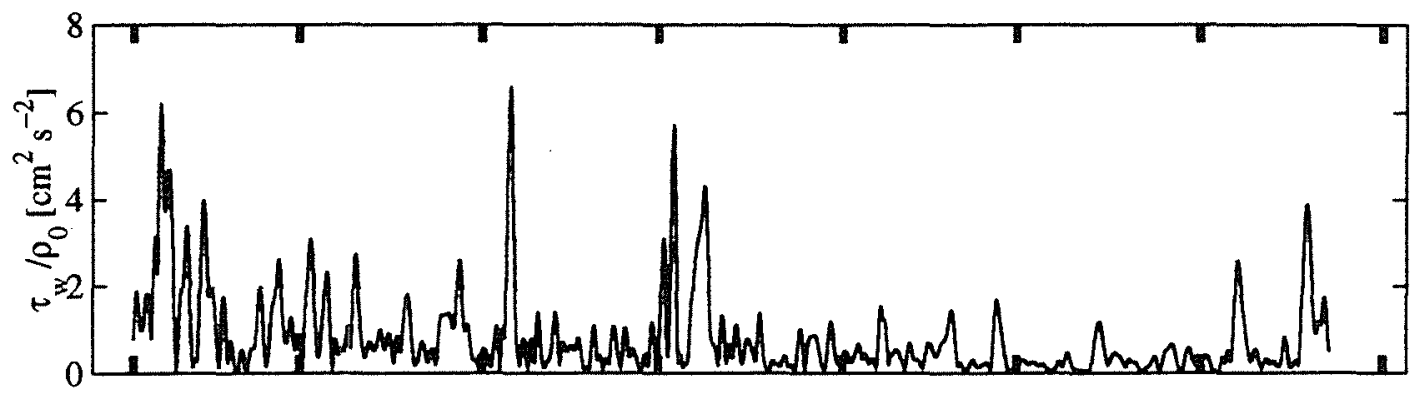

(c)

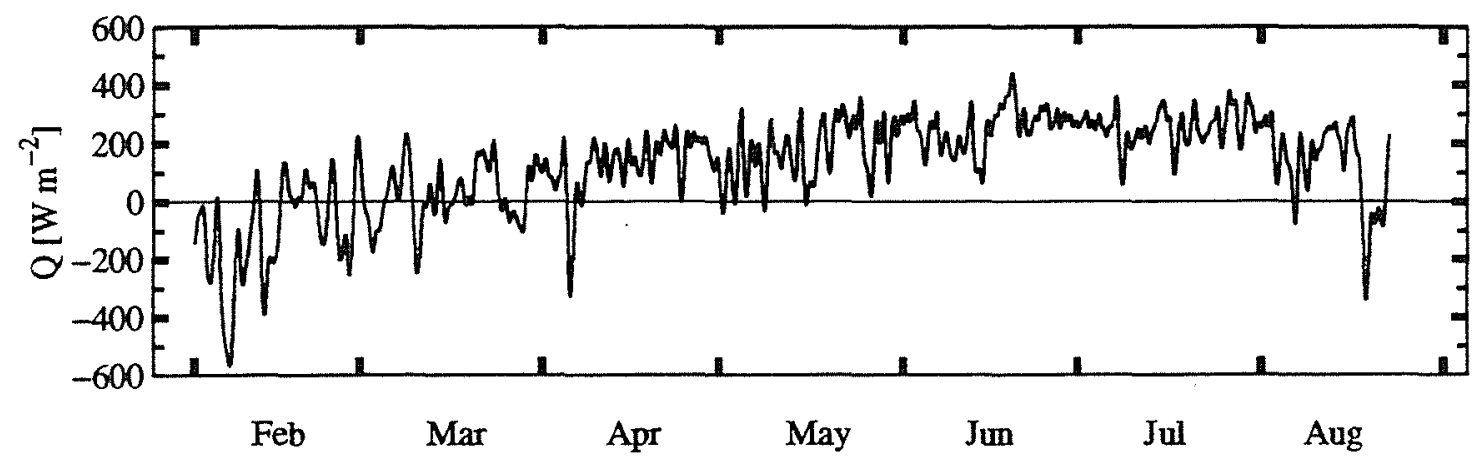

Fig.4.6: Hourly values of low-pass-filtered (a) mixed layer depth, (b) wind stress magnitude, and (c) net surface heat flux. The mixed layer depth is defined as the depth where the measured temperature deviates more than $0.05^{\circ}$ from observations at $1 \mathrm{~m}$ below the surface. 


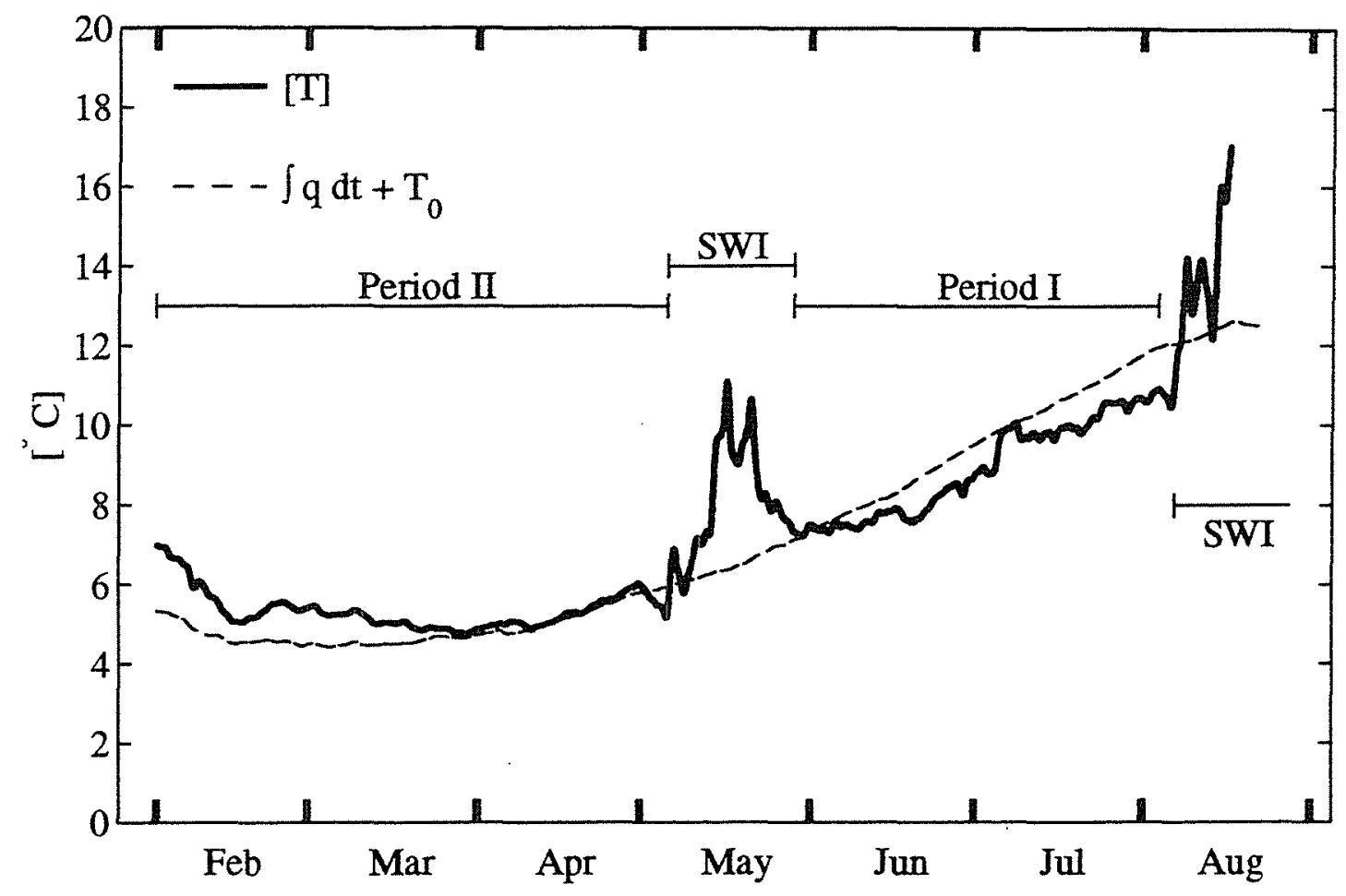

Fig.4.7: Time series of depth-averaged, low-pass-filtered (solid) temperature $[T]$ and (dashed) cumulative heat flux $\int q d t+T_{0}$, where $q=\frac{Q}{c_{p} \rho D}\left(Q\right.$ has units $\left.\mathrm{W} \mathrm{m}^{-2}, D=76 \mathrm{~m}\right) . T_{0}=5.3^{\circ} \mathrm{C}$ is an integration constant and was obtained from least-squares fit of $\int q d t$ to $[T]$, with measurements during the May and August SWIs excluded from the fit. 


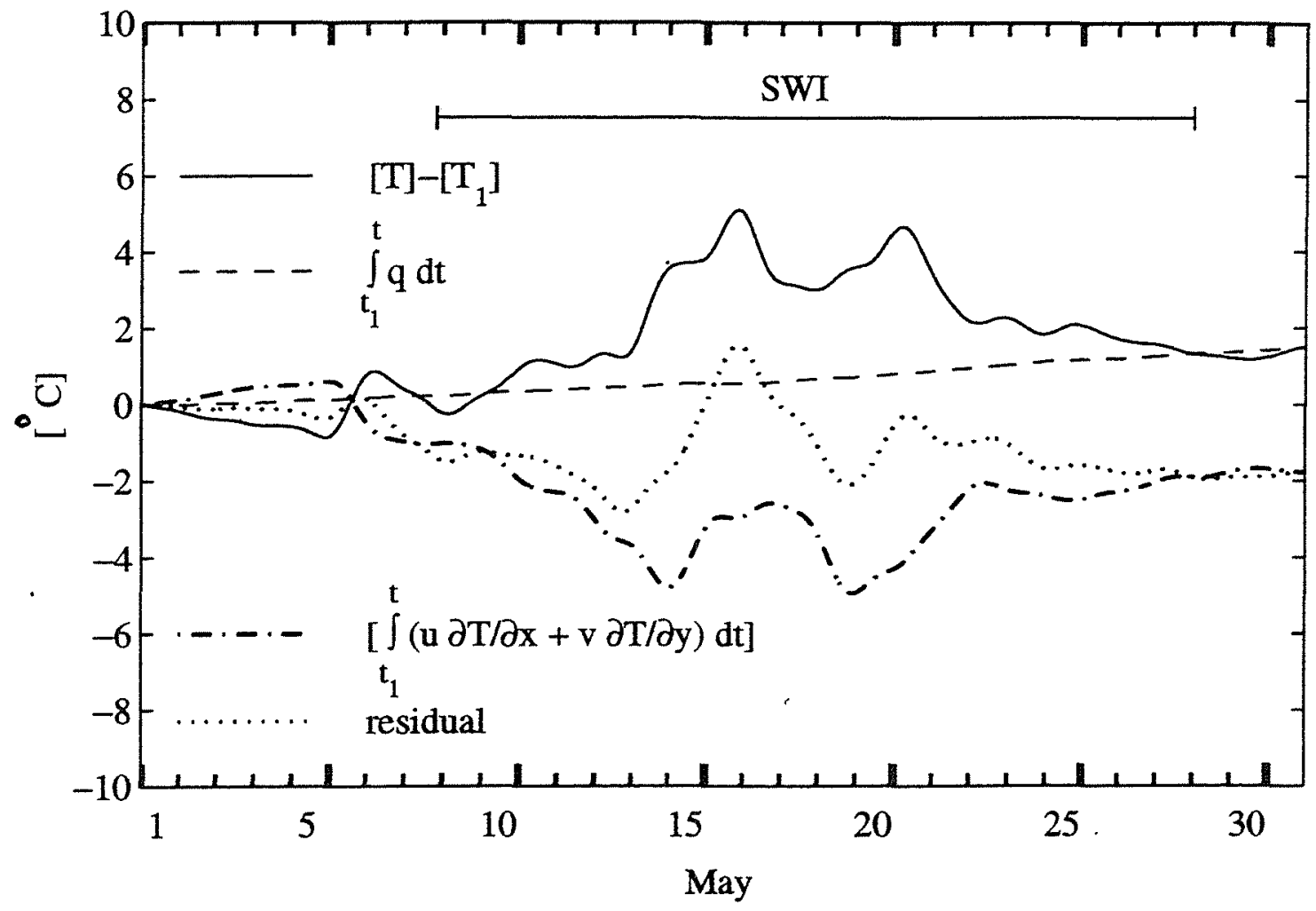

Fig.4.8: Hourly values of depth-averaged, low-pass-filtered (solid) temperature difference, (dashed) cumulative heat flux, (dot-dashed) cumulative heat transport, and (dotted) residual term during the May SWI. The starttime $t_{1}$ of the integration is midnight on May $1 .\left[T_{1}\right]=5.9^{\circ} \mathrm{C}$ corresponds to the depth-averaged temperature at $t=t_{1}$. 

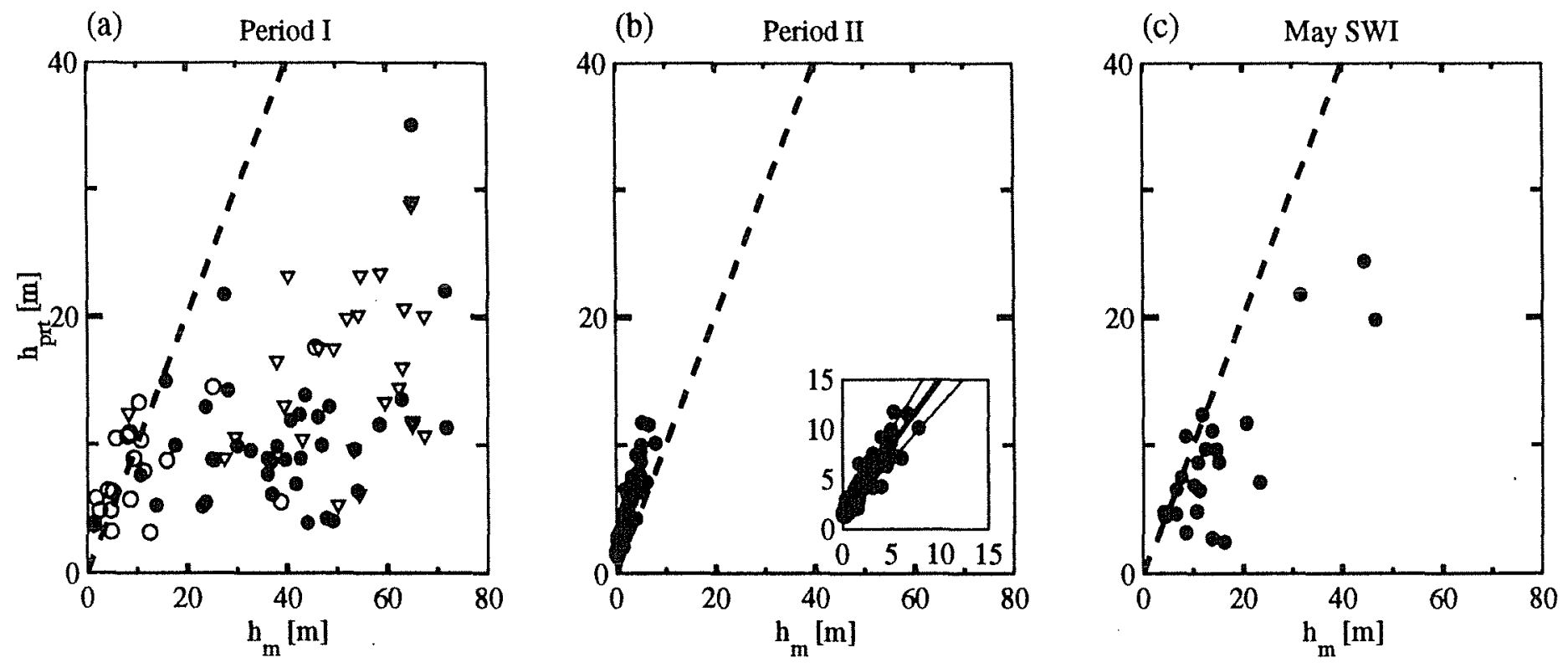

Fig.4.9: Daily averages of the predicted versus measured mixed layer depth for (a) February 1May 6 (period I), (b) May 29-August 4 (perdiod II), and (c) May 6-28 (SWI). Circles and diamonds denote times when the daily averaged surface heat flux was $\geq 0$ and $<0$, respectively. Open circles in (a) are data from April 10-30 when $95 \%$ of the daily averaged surface heat flux were within $162 \pm 112 \mathrm{~W} \mathrm{~m}^{-2}$. Dashed lines are reference lines that intersect the origin with slope 1.0. The enlargement in (b) shows the (heavy solid) linear regression between $h_{p r t}$ and $h_{m}$ with slope 1.3 and $y$-intersect $1.8 \mathrm{~m}$. Thin solid lines give the $95 \%$ confidence limits of the fit. Results are insensitive to whether or not a low-pass filter has been applied before the averaging. 
(a)

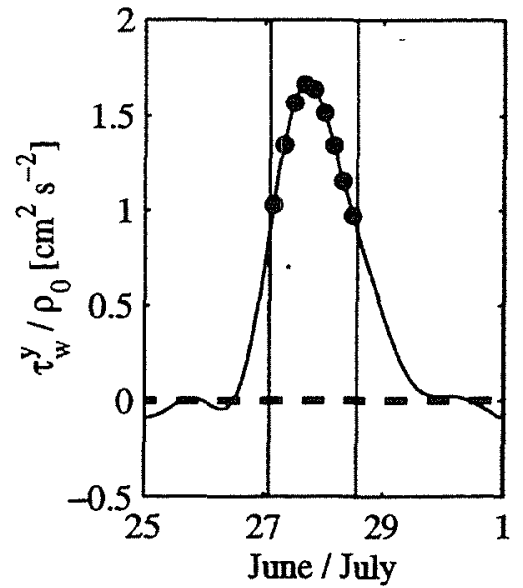

(b)



(c)

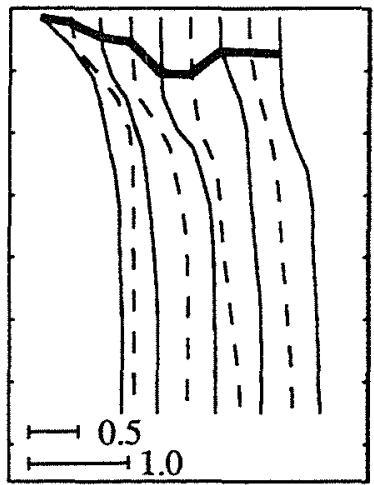

$\Delta \sigma_{\theta}$ (d)

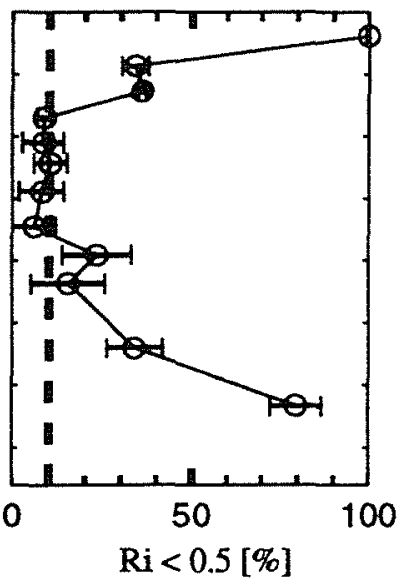

Fig.4.10: (a) Temporal evolution of the along-bank wind stress, (b) time-averaged, low-passfiltered velocity profiles, (c) vertical difference of potential density with respect to measurements at 1.5-m depth and (heavy solid) 4-hourly averaged mixed layer depth (each density profile represents a 4hour average with time progressing from left to right, solid and dashed lines are for clearer presentation), and (d) percentage of $R i<0.5$ for the wind event June 27-29. Vertical bars in (a) define the averaging period for (b) and (d) and were chosen such that $\frac{\tau_{W}^{W}}{\rho_{0}}>1 \mathrm{~cm}^{2} \mathrm{~s}^{-2}$. The cross-bank wind stress was nearly zero (Fig.4.1). Circles in (a) give the midpoints of the 4-hrs averaging intervals shown in (c). Estimates of $\sigma_{\theta}$ were obtained from SeaCAT and TPOD data, where salinity at the TPOD depths was computed using the vertically interpolated coefficients from linear T-S fits (see section 4.2.7 for details). Percentages $R i<0.5$ were determined from hourly averaged data as described in the text, with error bars giving the standard error at the 95\% significance level based on Monte Carlo simulations (see section 2.4.3 for details). The dashed line in (d) marks the cutoff criterion used to determine the vertical extent of the wind-driven circulation (see text). 


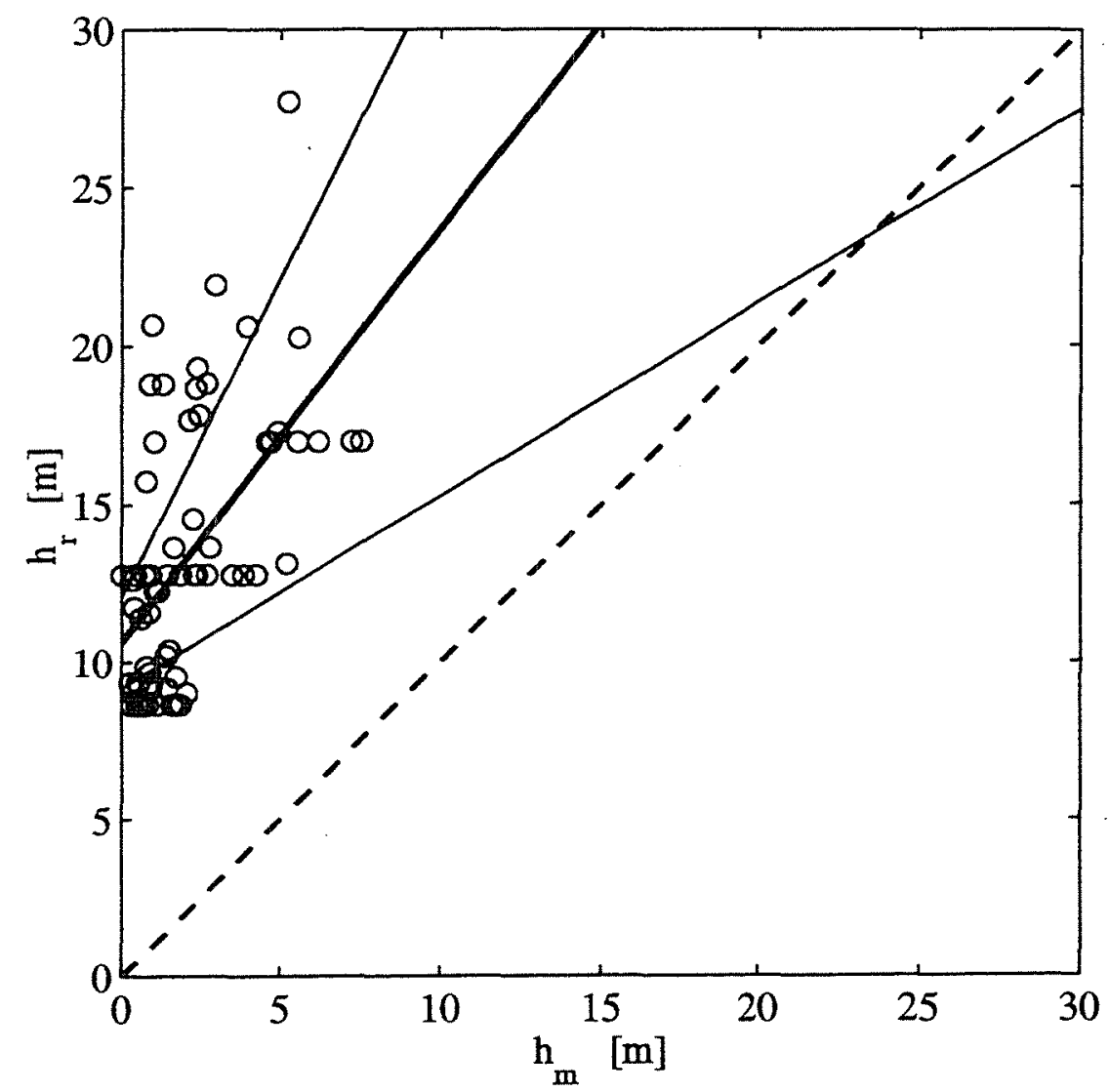

Fig.4.11: Daily averages of the reference depth $h_{r}$ versus the daily averaged mixed layer depth $h_{m}$ for period II. The dashd line is a reference line that intersects the origin with slope 1.0. Also shown is the (heavy solid) linear regression between $h_{r}$ and $h_{m}$ with slope 1.3 and $y$-intersect $10.6 \mathrm{~m}$. Thin solid lines give the $95 \%$ confidence limits of the fit. Results are insensitive to whether or not a low-pass filter has been applied before the averaging. 

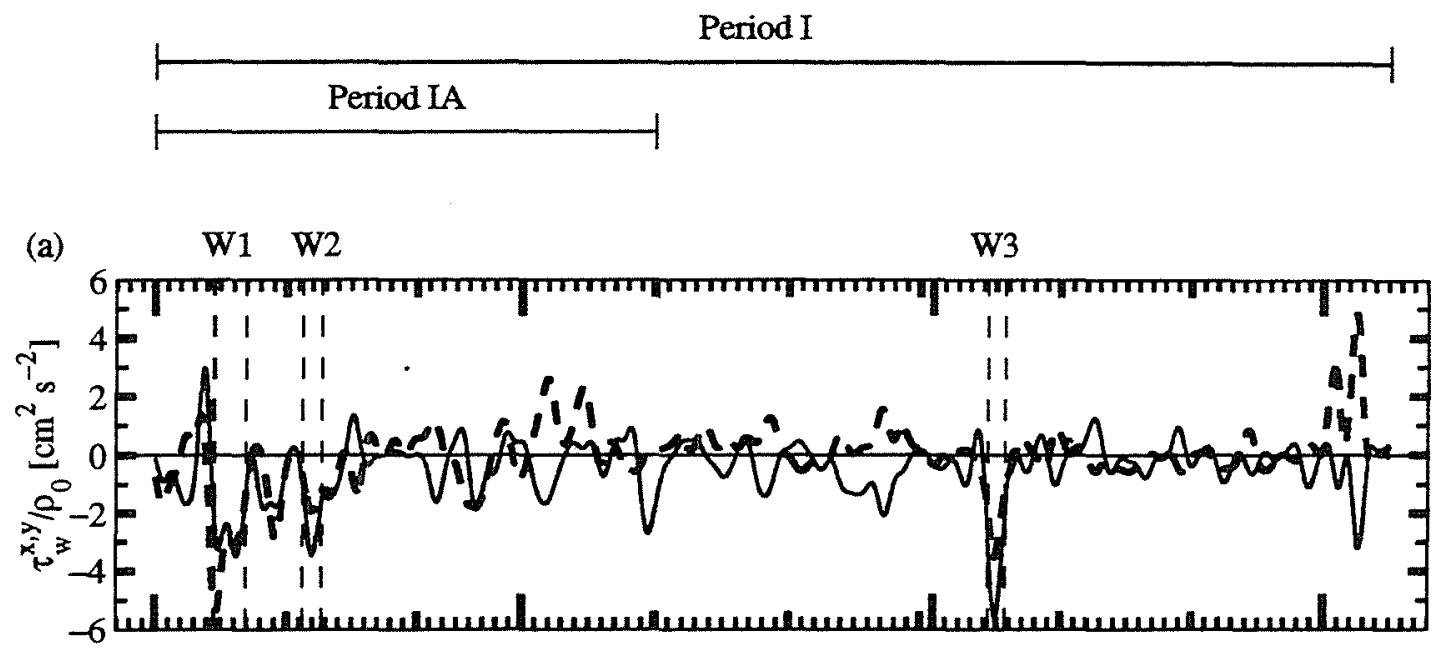

(b)

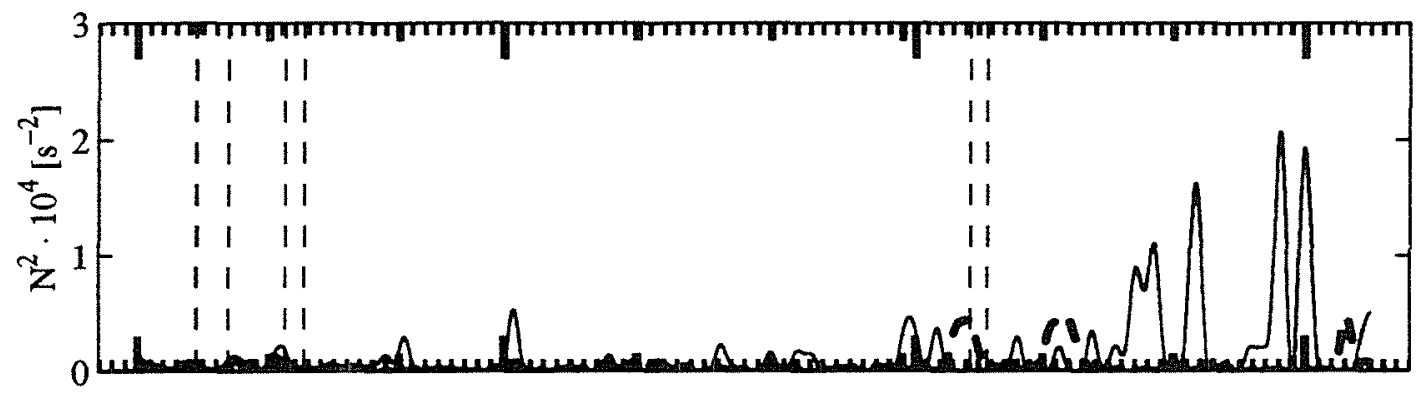

(c)

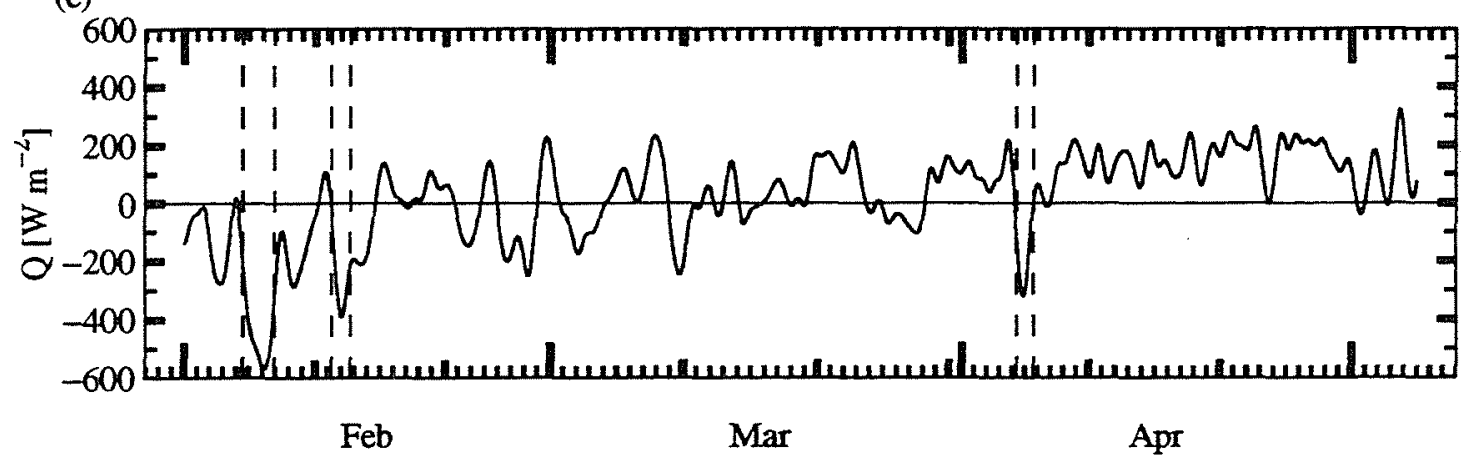

Fig.4.12: Hourly values of low-pass-filtered (a) (solid) cross- and (dashed) along-bank kinematic wind stress, (b) buoyancy frequency squared as in Fig.4.2, and (c) net surface heat flux as in Fig.4.5c for the investigation period I. Note that $N^{2}$ is shown on a different scale than in Fig.4.2. W1-3 mark the time intervals used for the event analysis in section 4.3.3. 


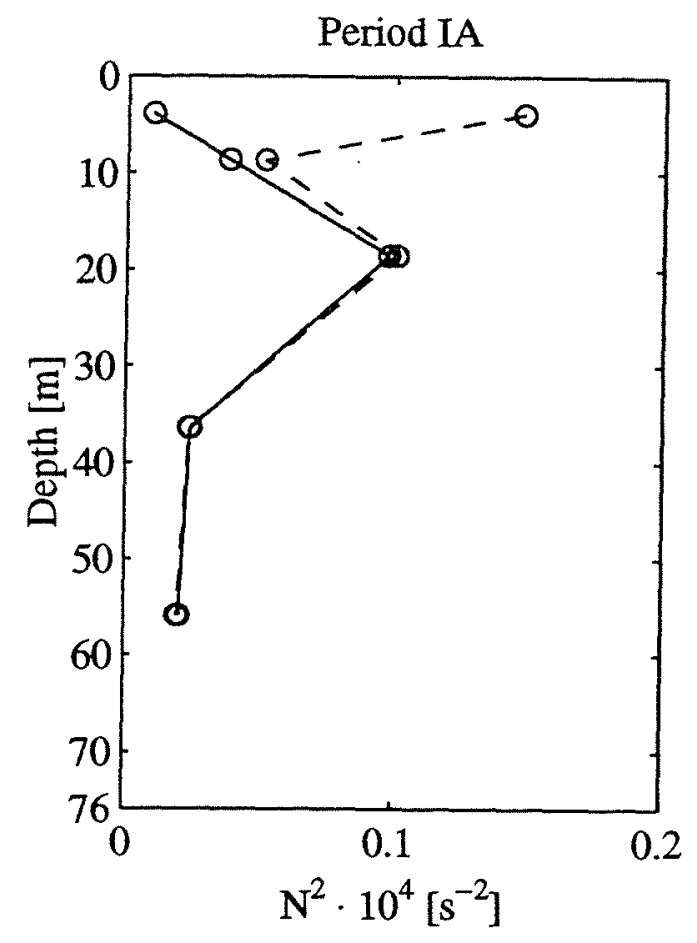

Fig.4.13: Time-mean buoyancy frequency squared for period IA (solid) without and (dashed) with intermittent intrusions of low salinity water included in the averaging. Low salinity water intrusions occured near the surface and covered about $11 \%$ of the investigation period. 


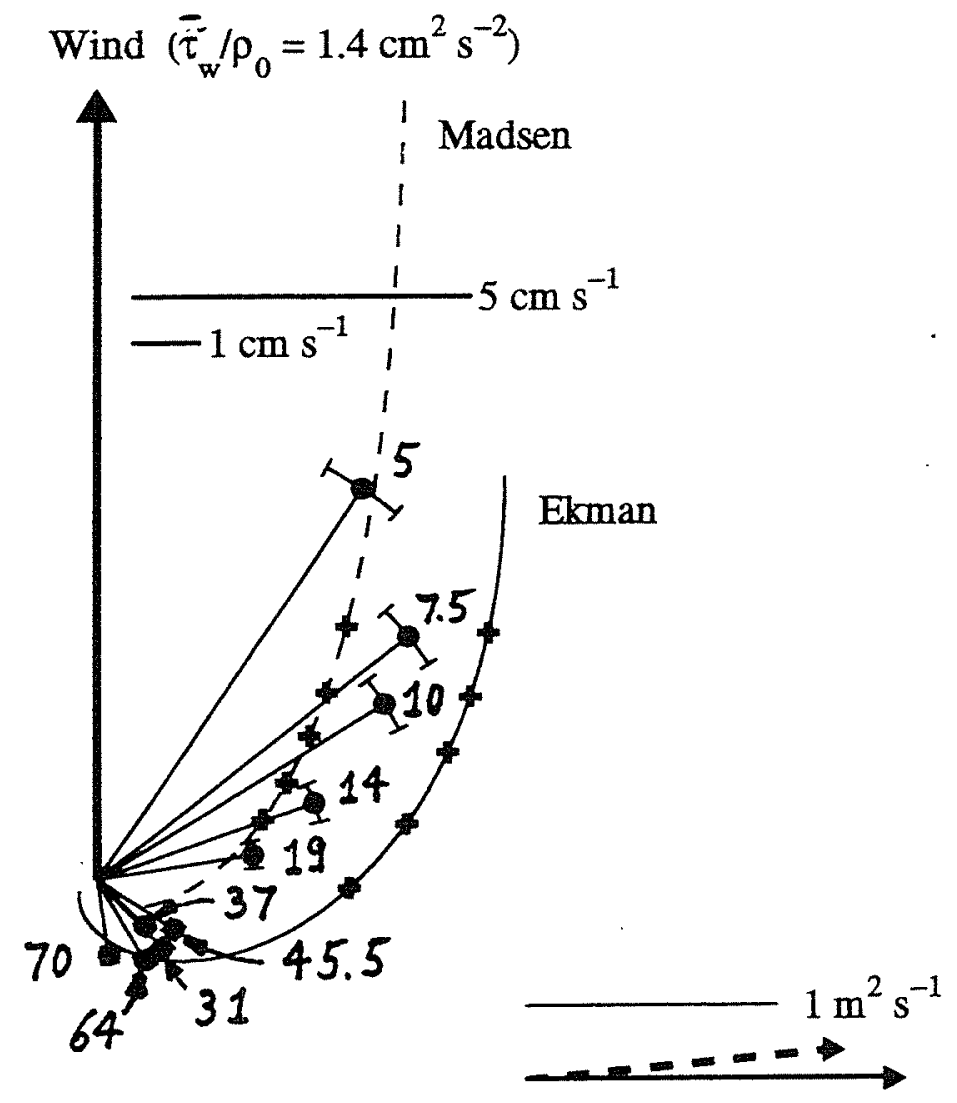

Fig.4.14: (Thin solid vectors) wind-induced currents $V_{W}$ for period IA from the "coherent ensemble average" method in relation to the (heavy vertical arrow) direction of the wind stress. $\frac{\bar{\tau}_{W}}{\rho_{0}}$ is the magnitude of the ensemble-averaged kinematic wind stress. Numbers denote VMCM depth, and curved error bars give the $\pm 5^{\circ}$ compass uncertainties. Also shown are (solid) the Ekman solutions for $\frac{\bar{\tau}_{W W}}{\rho_{0}}=1.4 \mathrm{~cm}^{2} \mathrm{~s}^{-2}$ and $D_{E}=22 \mathrm{~m}$ at 0-76-m depth, and (dashed) results from Madsen's model at 0.3-76-m depth. Above 0.3-m depth, Madsen's model predicts the current shear is almost strictly dowawind with surface velocities near $30 \mathrm{~cm} \mathrm{~s}^{-1}$ (not shown). Plusses mark analytical solutions at the instrumentation depths of the upper five VMCMs (5-19 m). Heavy arrows in the lower right corner represent (solid) the Ekman transport $\frac{\bar{T}_{W}}{\rho_{0} f}$ and (dashed) the estimated transport based on integration of $\mathbf{V}_{W}$ over the water depth. 



Fig.4.15: Hourly values of (top) magnitude of the low-pass-filtered wind stress vector, (middle) low-pass-filtered wind direction (positive counterclockwise, with $0^{\circ}$ defining the on-bank axis), and (bottom,solid) unfiltered and (dashed) low-pass-filtered net surface heat flux for W1-3. Vertical bars define the time window for the event analysis. 

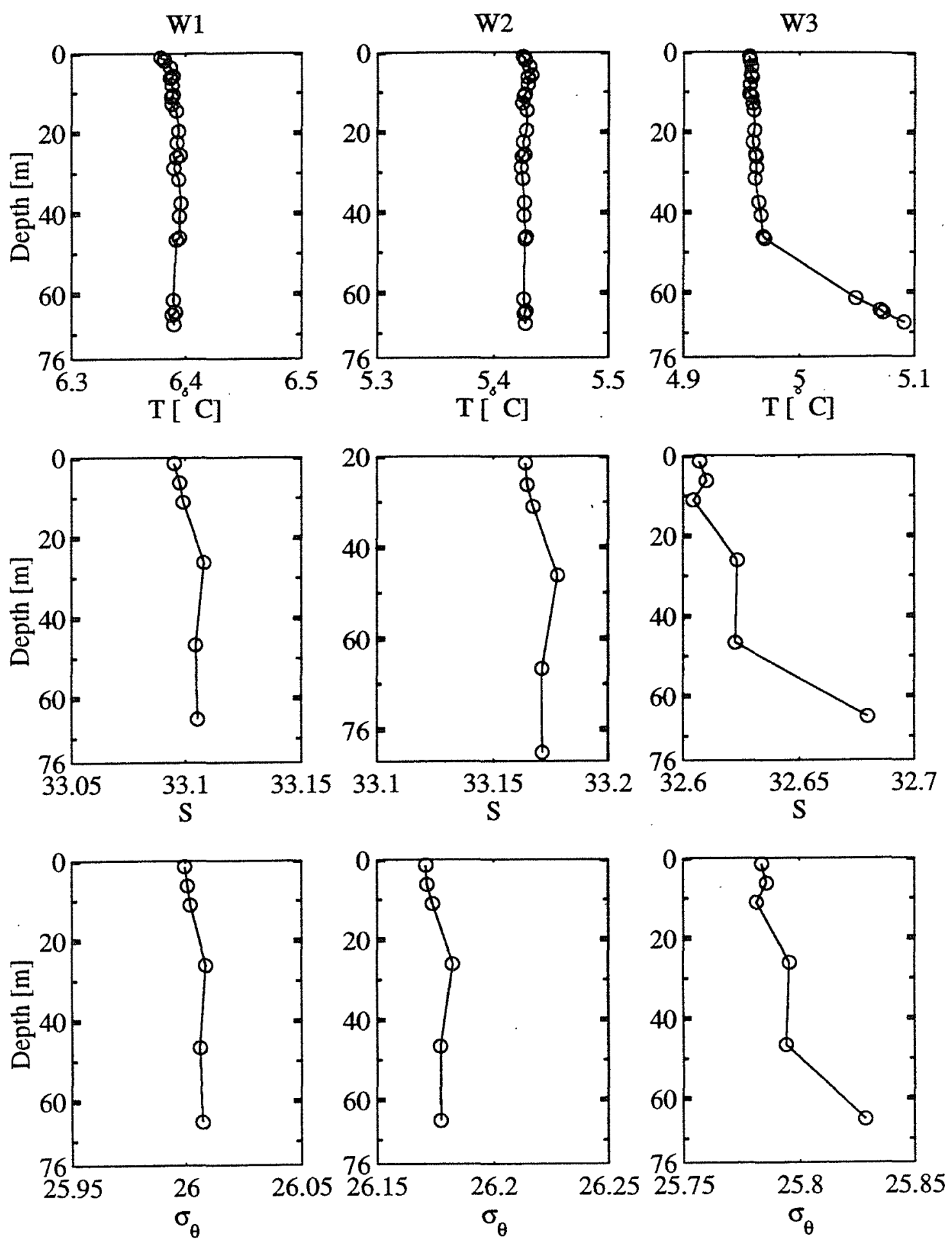

Fig.4.16: Profiles of event-averaged (top) temperature, (middle) salinity, and (bottom) potential density. 
(a) Wind $\left(\bar{\tau}_{w} / \rho_{0}=4.4 \mathrm{~cm}^{2} \mathrm{~s}^{-2}\right)$

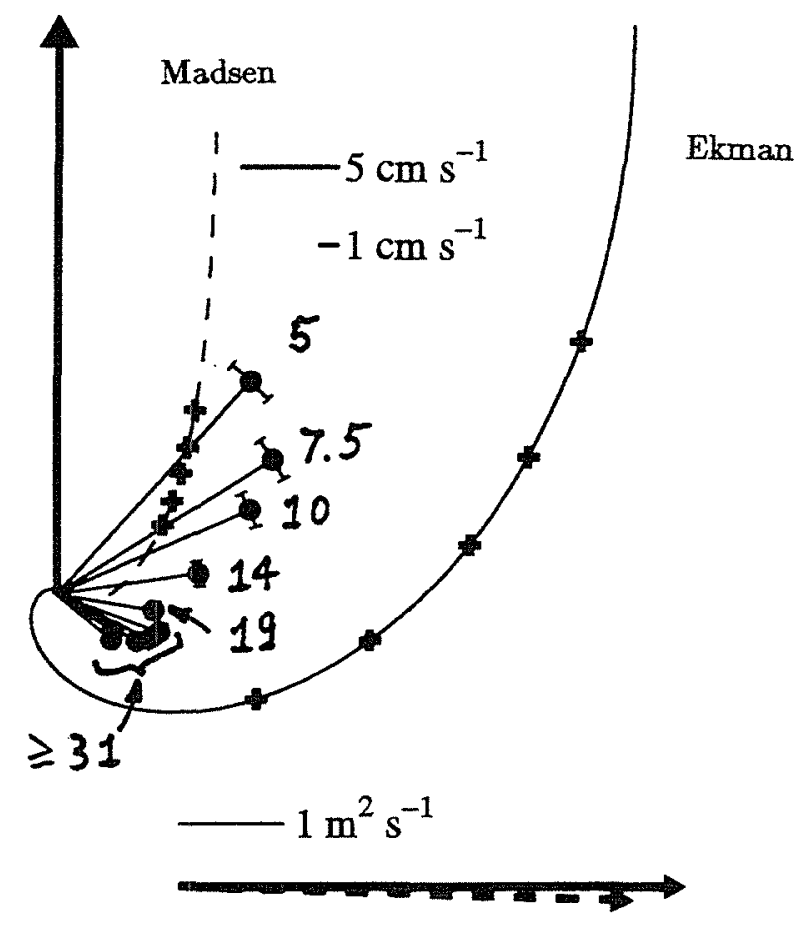

Fig.4.17a: Event-averaged results for W1, presented as in Fig.4.14, but note that the scales are different. Ekman solutions are shown between 0-76-m depth for $\frac{\bar{\tau}_{W X}}{\rho_{0}}=4.4 \mathrm{~cm}^{2} \mathrm{~s}^{-2}$ and $D_{E}=L_{\theta}=15 \mathrm{~m}$. Results from Madsen's model are depicted at $0.3-76-\mathrm{m}$ depth. Surface velocities from Madsen's model are about twice the predicted value at 0.3-m depth (not shown). 
(b) Wind $\left(\bar{\tau}_{\mathrm{w}} / \rho_{0}=3 \mathrm{~cm}^{2} \mathrm{~s}^{-2}\right)$



Fig.4.17b: Event-averaged results for W2 presented as in Fig.4.14, but note that the scales are different. Ekman solutions are shown between 0-76-m depth for $\frac{\bar{\tau}_{W}}{\rho_{0}}=3.0 \mathrm{~cm}^{2} \mathrm{~s}^{-2}$ and $D_{E}=L_{\theta}=14 \mathrm{~m}$ Results from Madsen's model are depicted at 0.3-76-m depth. Surface velocities from Madsen's model are about twice the predicted value at 0.3-m depth (not shown). 
(c) Wind $\left(\bar{\tau}_{w} / \rho_{0}=5.1 \mathrm{~cm}^{2} \mathrm{~s}^{-2}\right)$

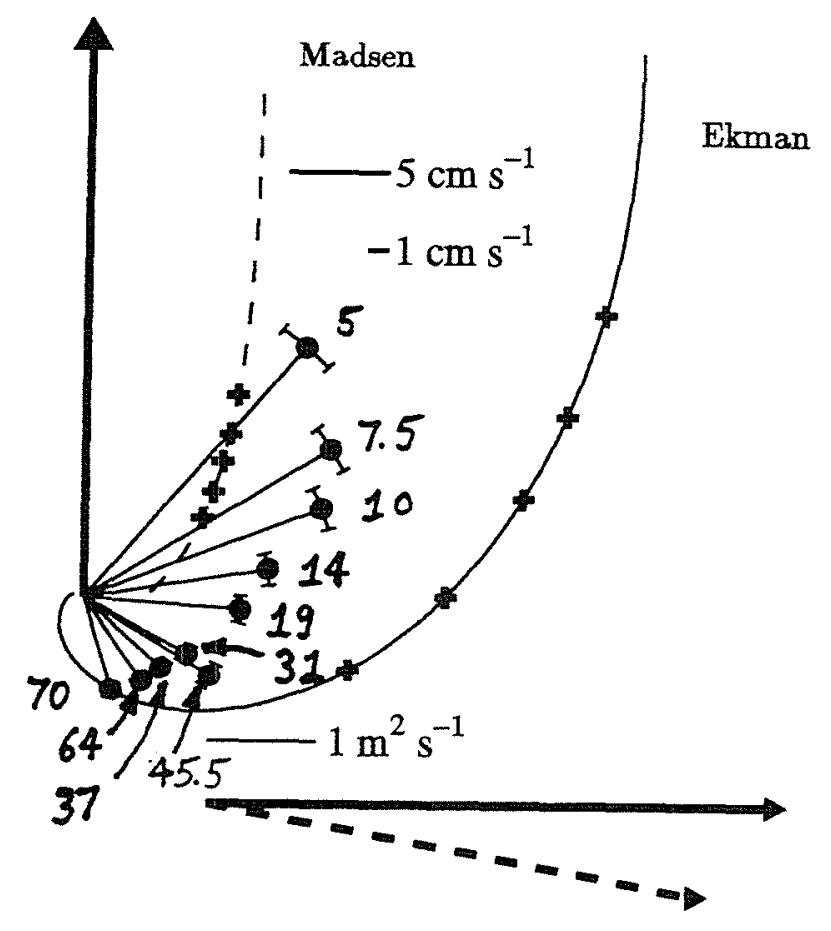

Fig.4.17c: Event-averaged results for W3 presented as in Fig.4.14, but note that the scales are different. Ekman solutions are shown between 0-76-m depth for $\frac{\bar{T}_{W}}{\rho_{0}}=5.1 \mathrm{~cm}^{2} \mathrm{~s}^{-2}$ and $D_{E}=L_{\theta}=19 \mathrm{~m}$. Results from Madsen's model are depicted at 0.3-76-m depth. Surface velocities from Madsen's model are about twice the predicted value at 0.3 -m depth (not shown). 

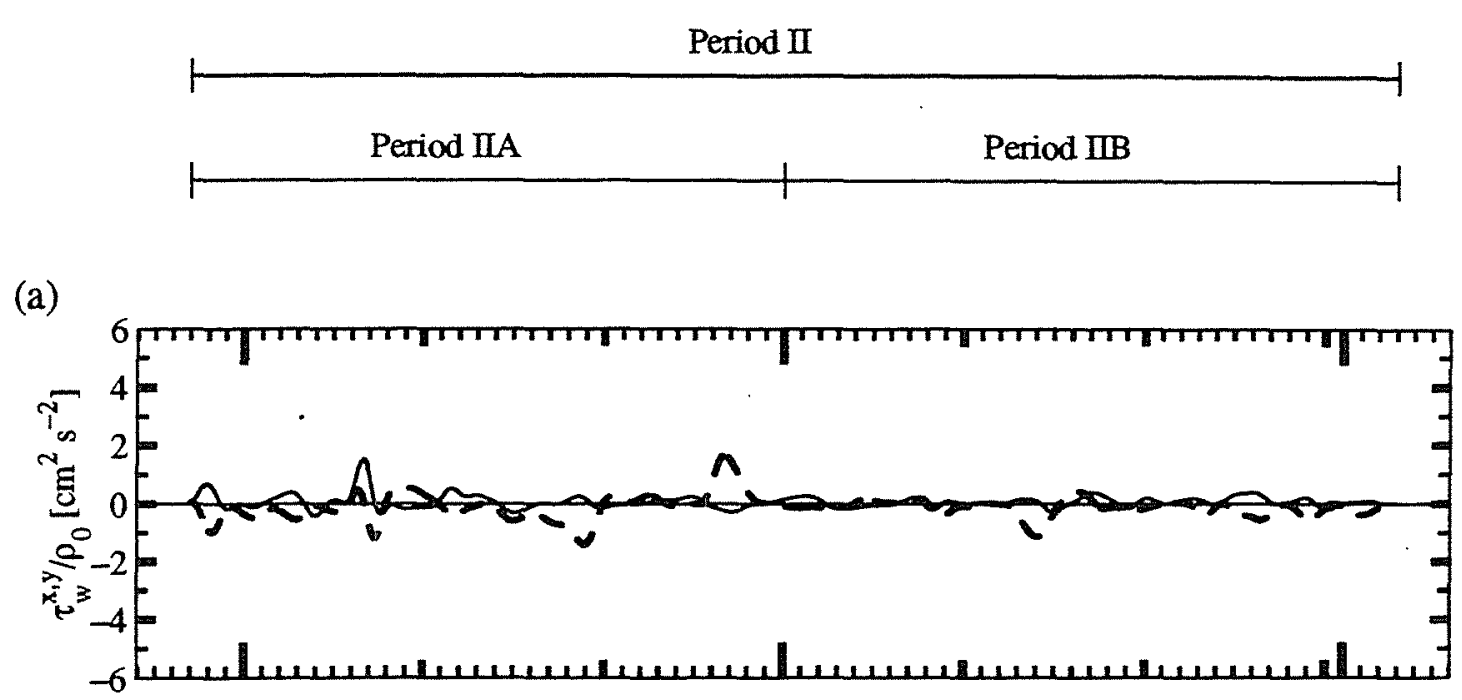

(b)
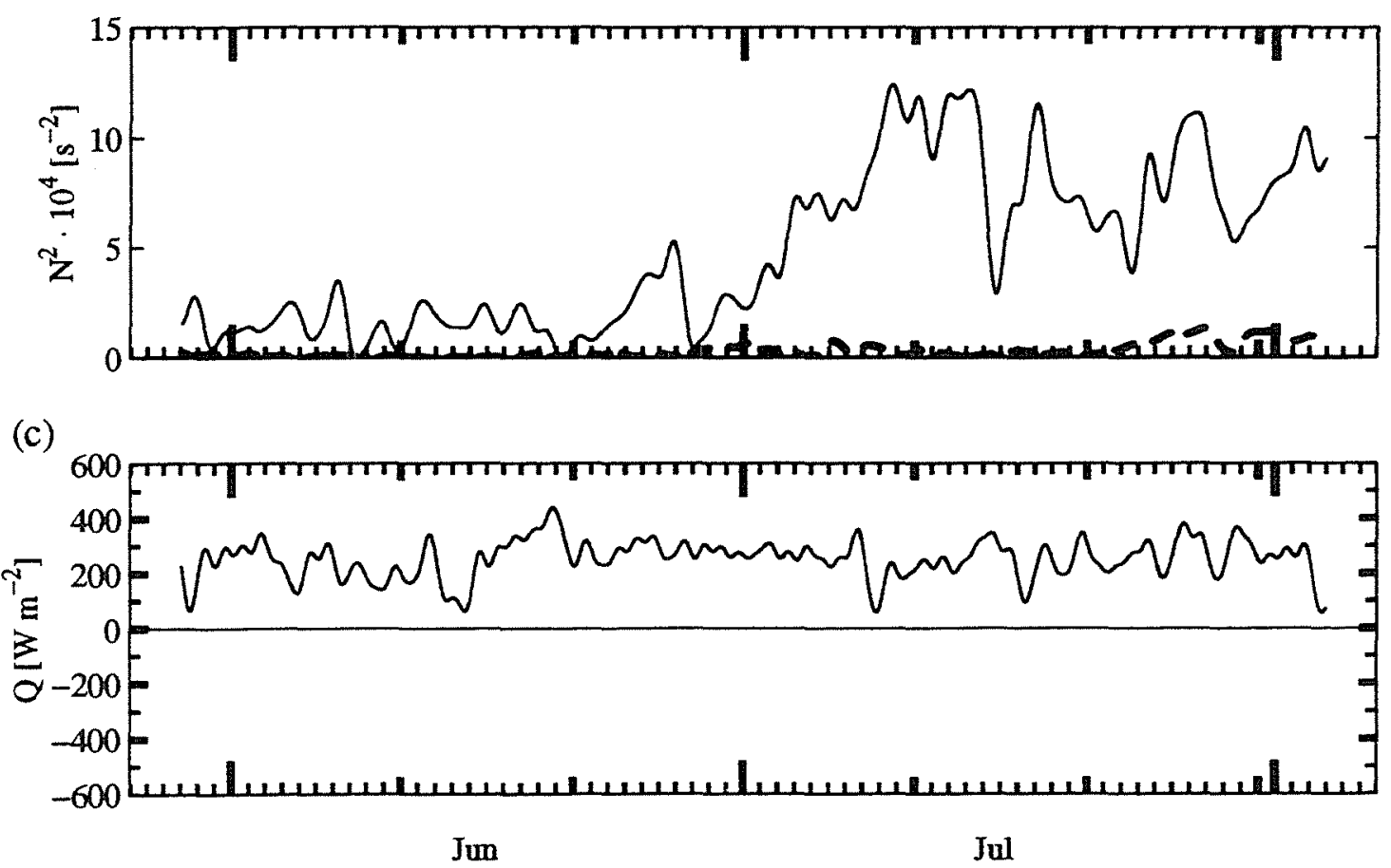

Fig.4.18: Hourly values of low-pass-filtered (a) (solid) cross- and (dashed) along-bank kinematic wind stress, (b) buoyancy frequency squared as in Fig.4.3, and (c) net surface heat flux as in Fig.4.6c for the investigation period II. 


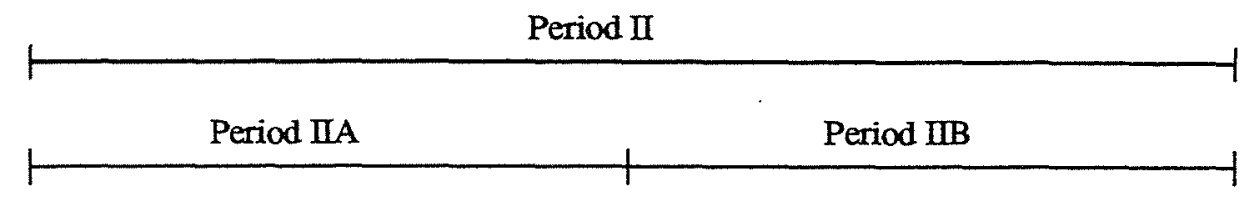

(a)

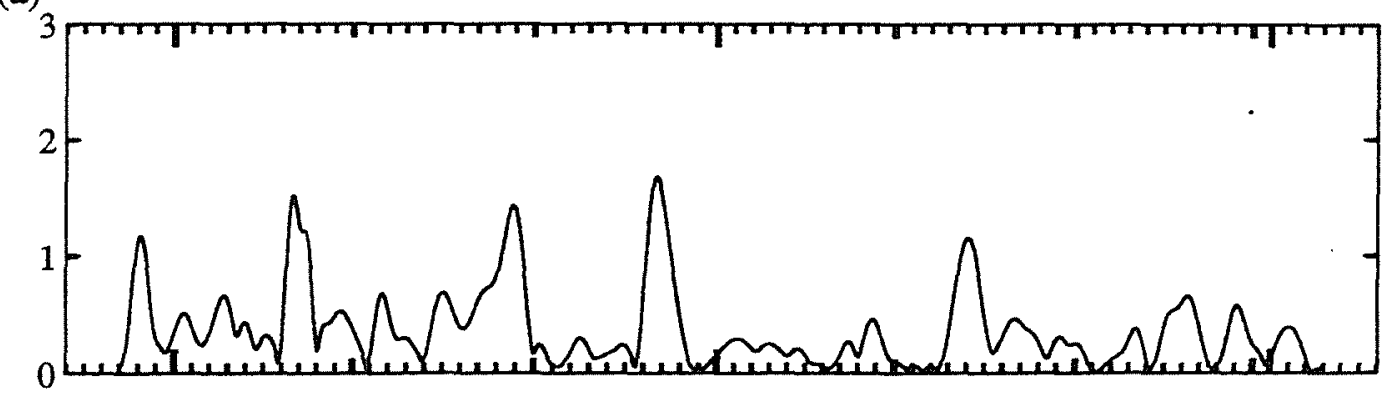

(b)



(c)

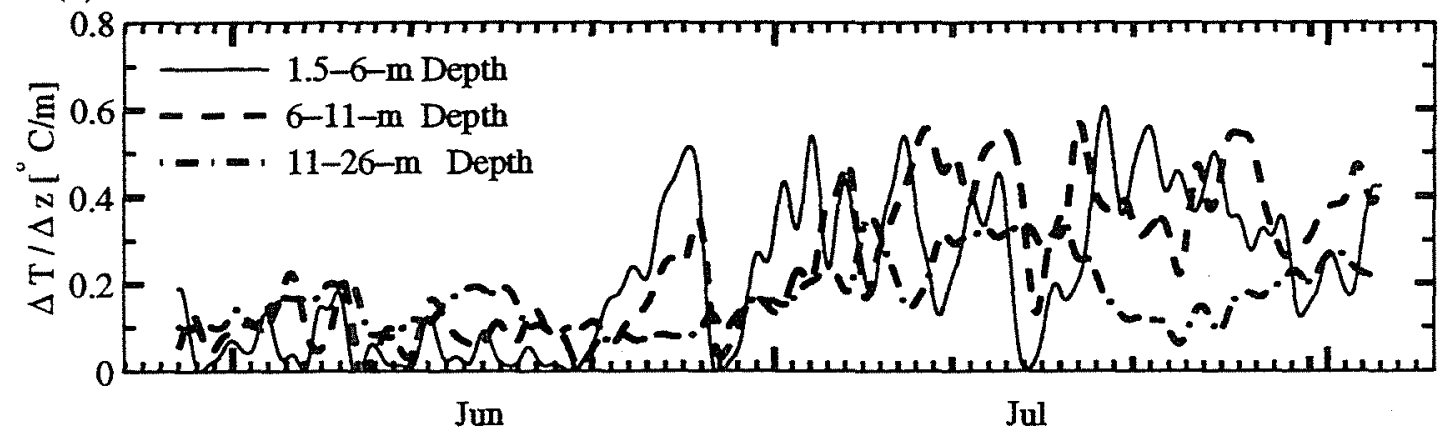

Fig.4.19: Hourly values of the low-pass-filtered (a) kinematic wind stress magnitude, (b) buoyancy frequency squared, and (c) thermal stratification near the surface for period II. Panels (b) and (c) are from SeaCAT data at 1.5, 6, 11, and 26-m depth. 
(a) Wind $\left(\tilde{\tau}_{w} / \rho_{0}=0.5 \mathrm{~cm}^{2} \mathrm{~s}^{-2}\right)$

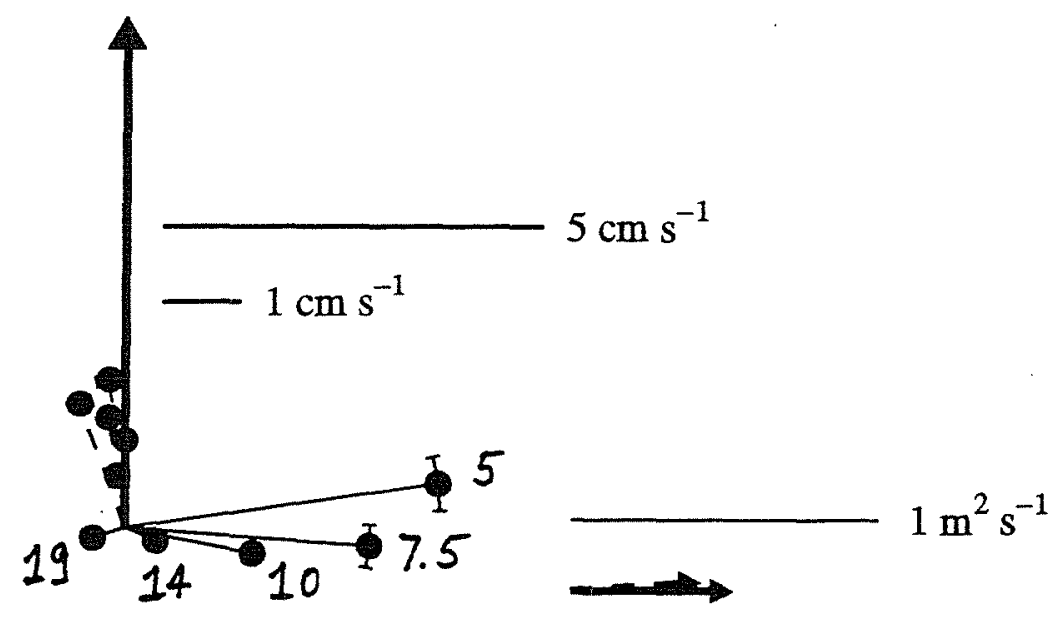

(b) Wind $\left(\tau_{w} / \rho_{0}=0.3 \mathrm{~cm}^{2} \mathrm{~s}^{-2}\right)$



Fig.4.20: Ensemble-averaged, wind-induced currents and transport vectors for (a) period ILA and (b) period IIB as in Fig.4.14, but without the Ekman and Madsen solutions. Dashed vectors represent velocities at 31-m depth and below. The integration depth of the transport vector varied with time according to the specified reference depth (see text). 

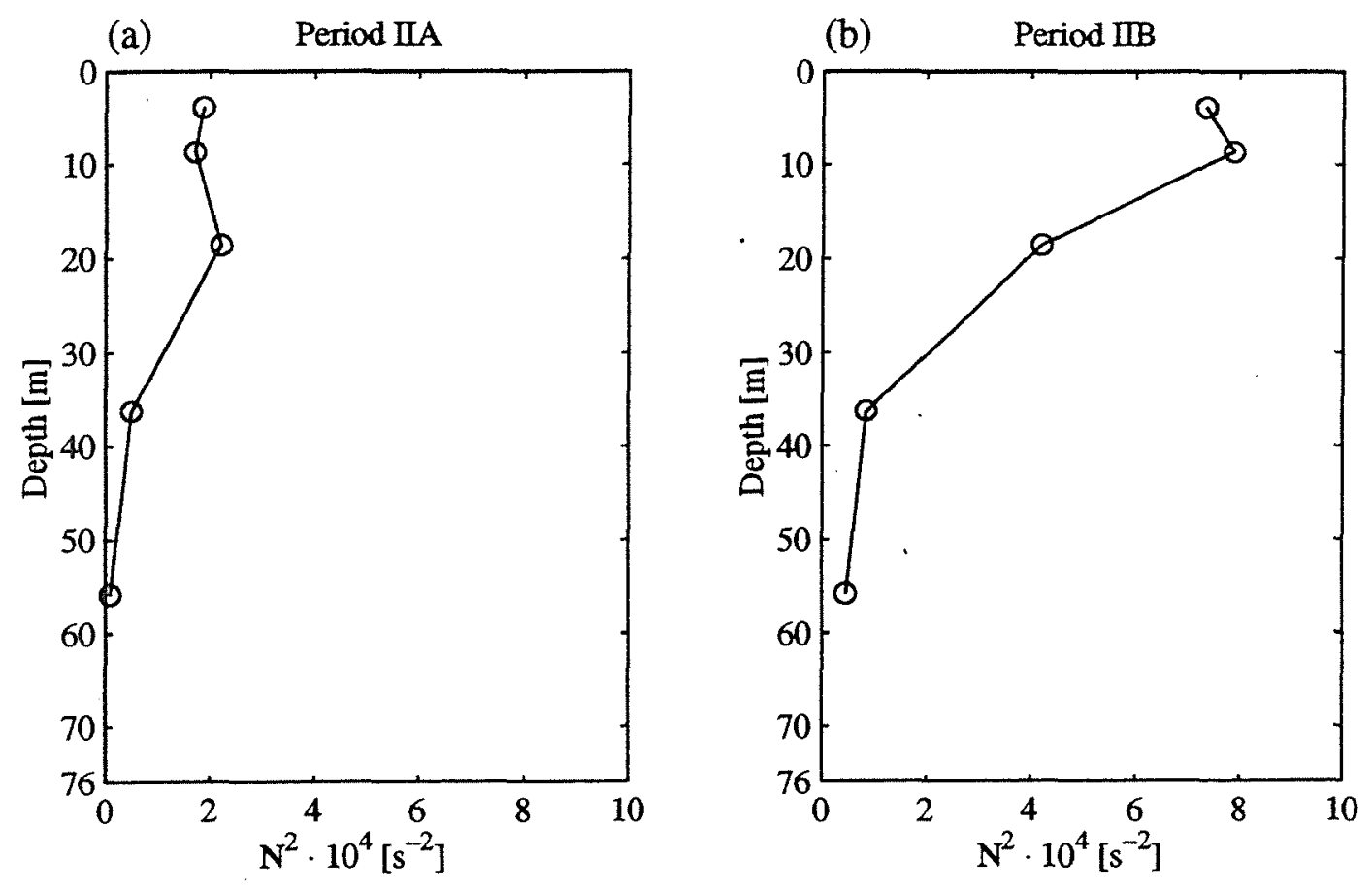

Fig.4.21: Time-mean buoyancy frequency squared for (a) period IIA and (b) period IIB. 



Fig.4.22: Hourly values of unfiltered net surface heat flux for period II. 
(a) Period IIA


Fig.4.23a: Mean diumal variation of (top) net surface heat flux and (middle and bottom) mixed layer depth for period IIA with $\left(h_{D}\right)$ and without $\left(h_{m}\right)$ the low-pass filter of $h_{m}$ removed from the measurements. Each day was divided into 24 bins, and unfiltered, hourly estimates of $Q, h_{m}$, and $h_{D}$ were averaged to obtain one representative diurnal cycle. Positive $h_{D}$ describes mixed layer deepening. Dashed lines give the $95 \%$ probability distribution of the standard error. 
(b)

Period IIIB
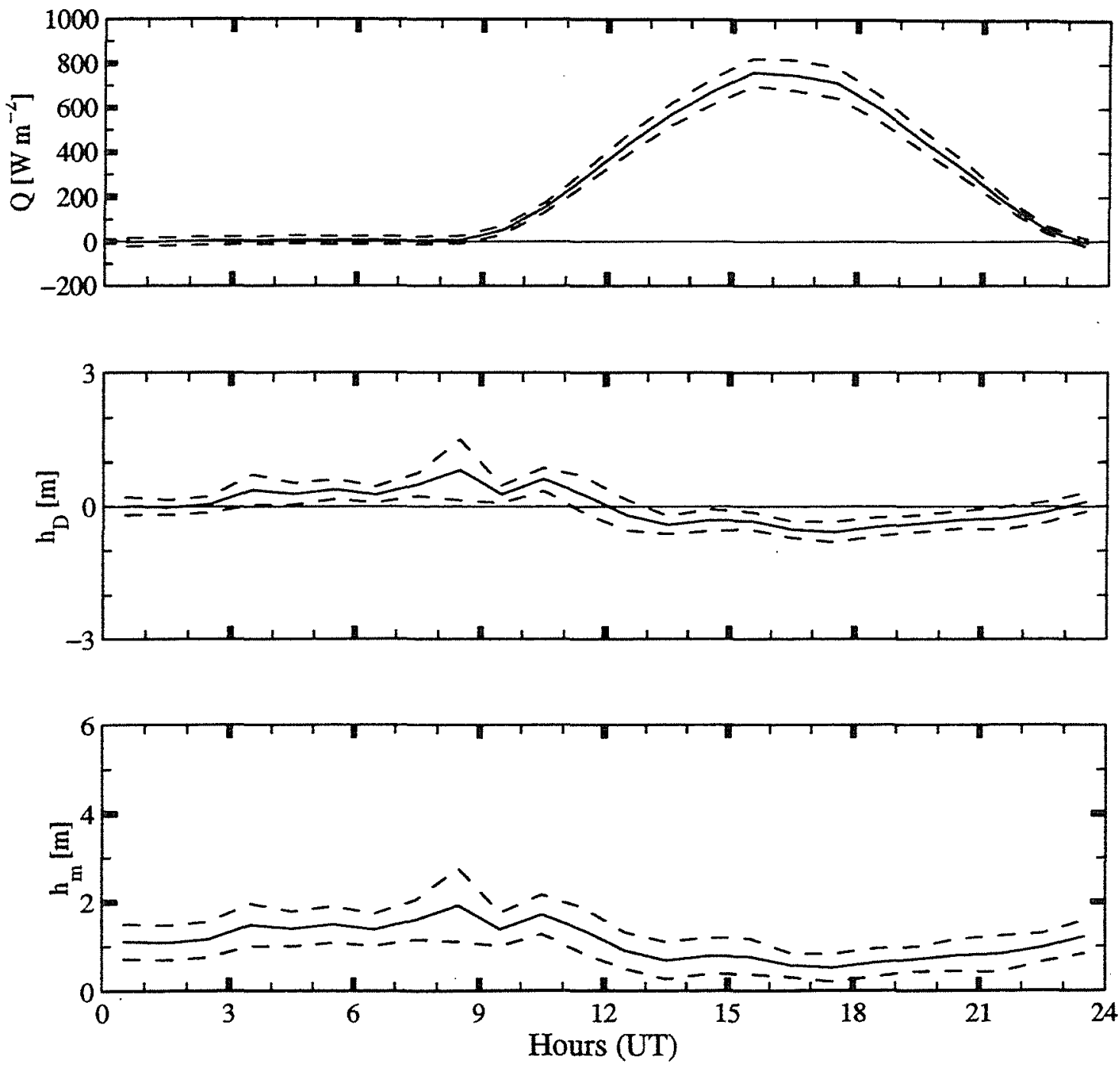

Fig.4.23b: Mean diurnal variation of (top) net surface heat flux and (middle and bottom) mixed layer depth for period IIB with $\left(h_{D}\right)$ and without $\left(h_{m}\right)$ the low-pass filter of $h_{m}$ removed from the measurements. Each day was divided into 24 bins, and unfiltered, hourly estimates of $Q, h_{m}$, and $h_{D}$ were averaged to obtain one representative diurnal cycle. Positive $h_{D}$ describes mixed layer deepening. Dashed lines give the $95 \%$ probability distribution of the standard error. 
(a)

Period IIA
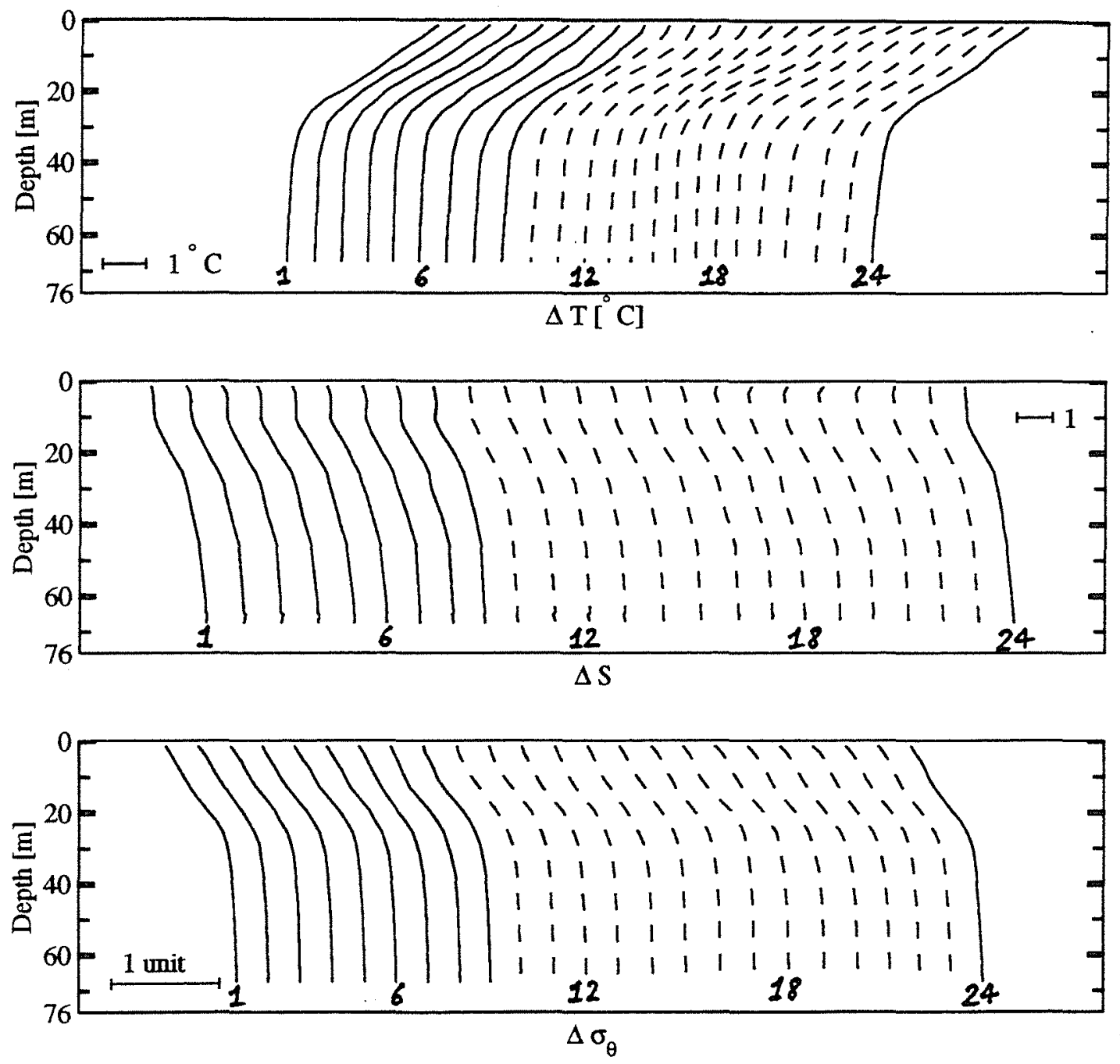

Fig.4.24a: Mean diurnal cycle of (top) temperature, (middle) salinity, and (bottom) potential density shown as difference from the measurements at $1.5-\mathrm{m}$ depth for period ILA. Profiles were determined following the time-averaging procedure described in Fig.4.23. Dashed and solid lines correspond to nighttime (24-9 hrs UT) and daytime (10-23 hrs UT) data, repectively. Numbers 1-24 give the hour for which the average was taken. 
(b)

Period IIB
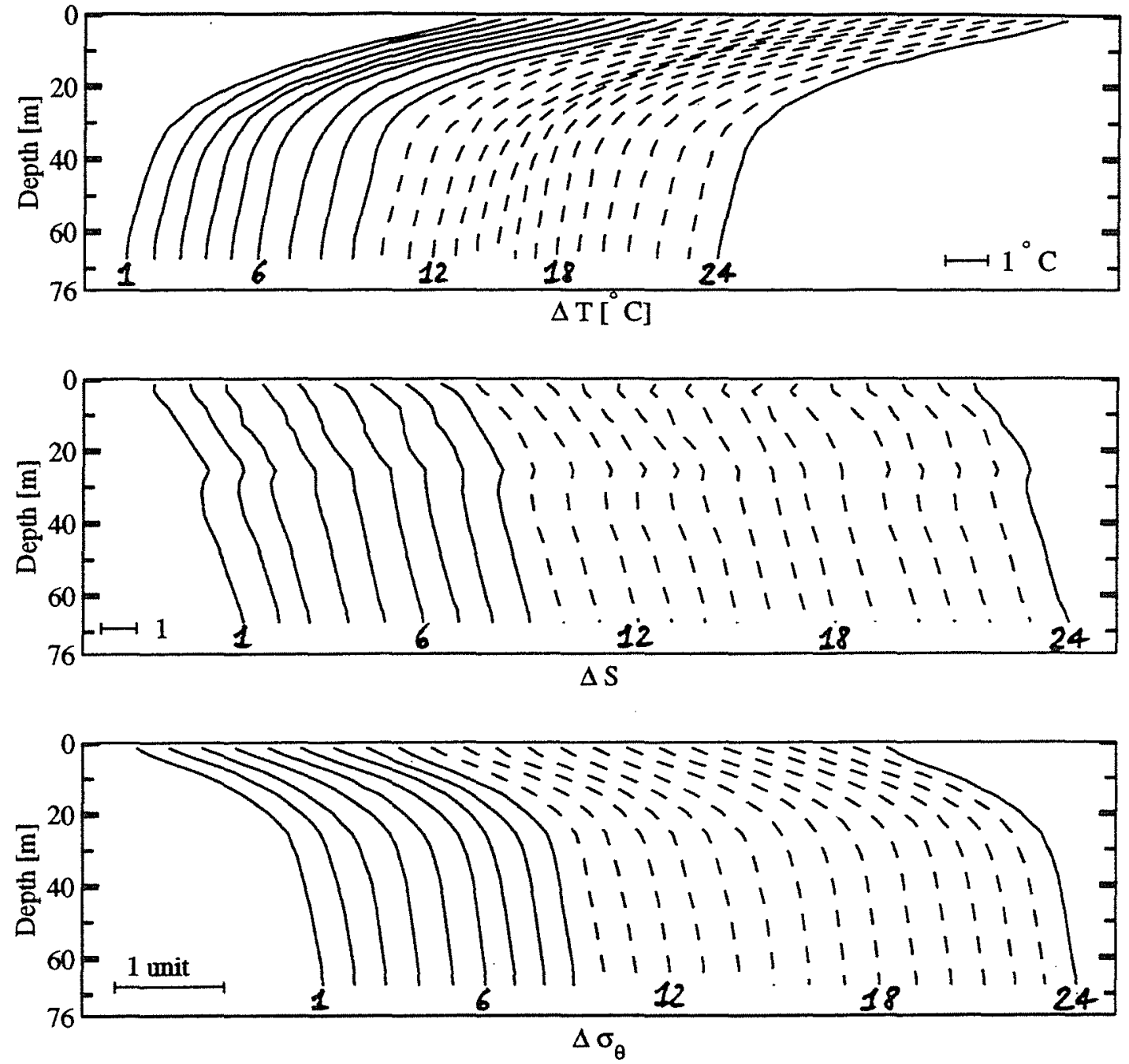

Fig.4.24b: Mean diurnal cycle of (top) temperature, (middle) salinity, and (bottom) potential density shown as difference from the measurements at 1.5-m depth for period IIB. Profiles were determined following the time-averaging procedure described in Fig.4.23. Dashed and solid lines correspond to nighttime (24-9 hrs UT) and daytime (10-23 hrs UT) data, repectively. Numbers 1-24 give the hour for which the average was taken. 
(a)

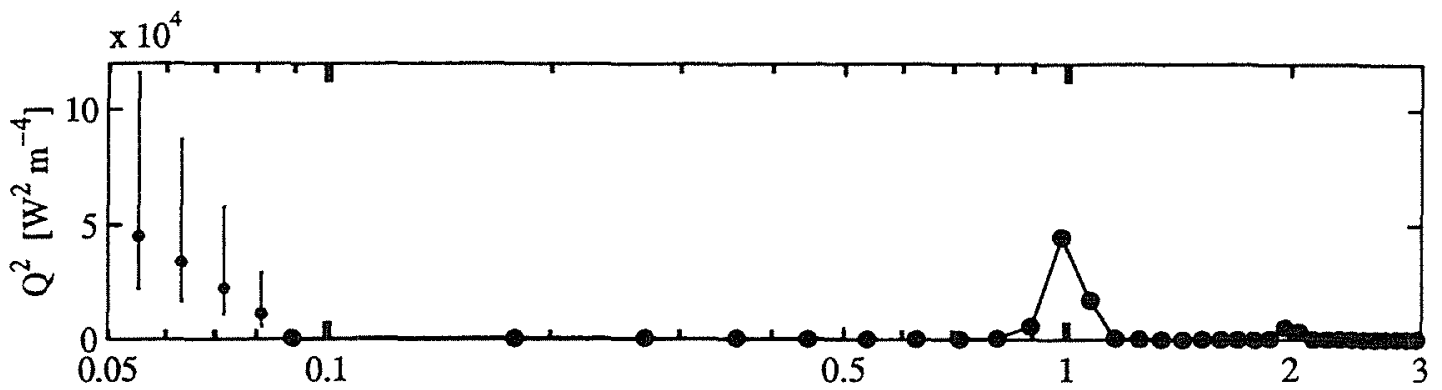

(b)

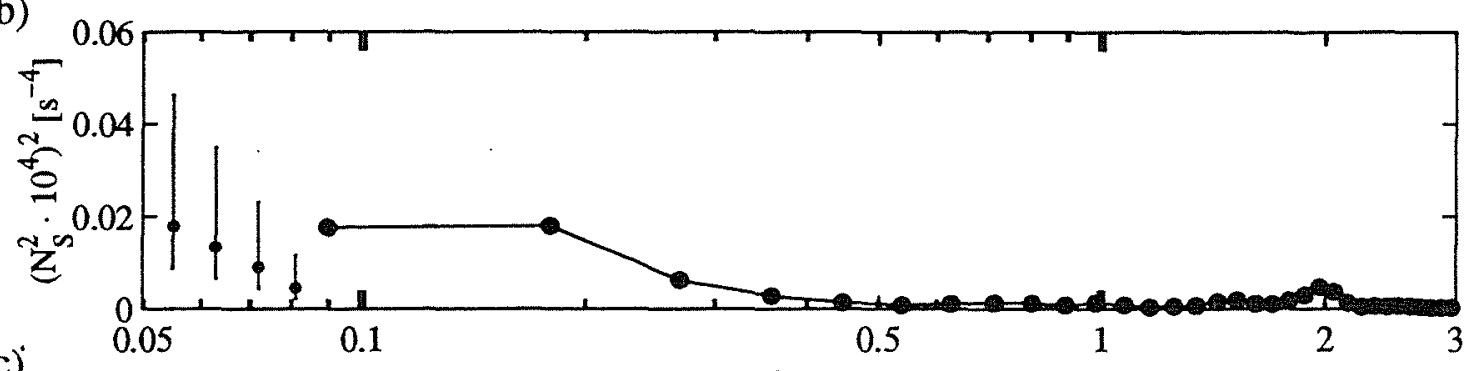

(c)

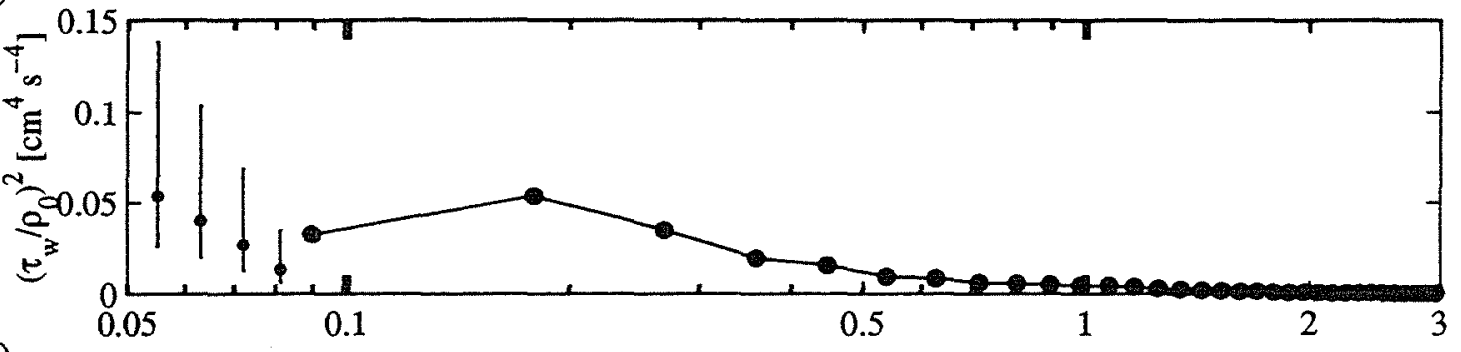

(d)

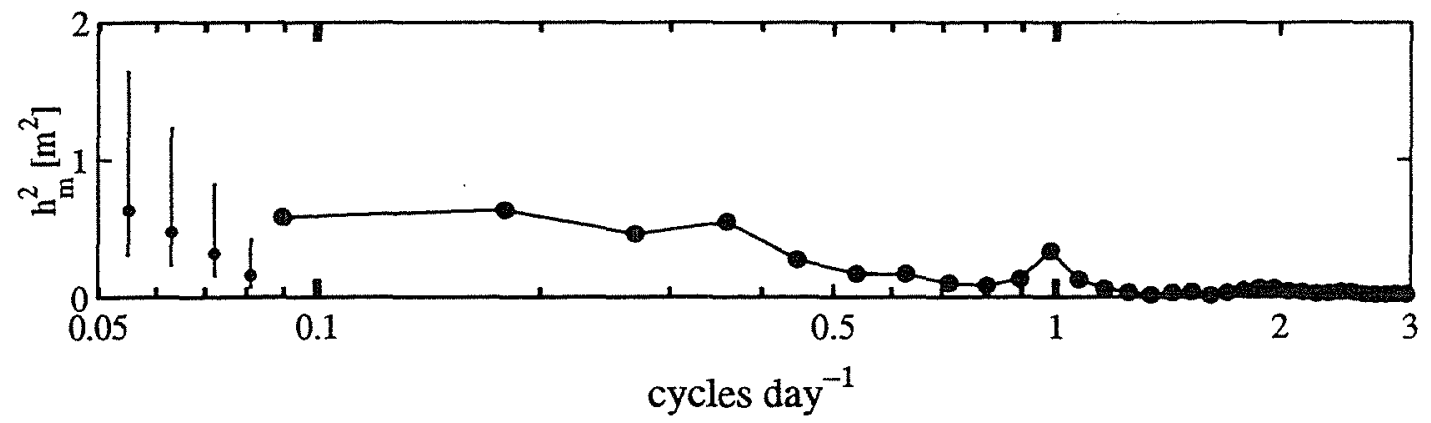

Fig.4.25: Variance conserving spectra performed on unfiltered, hourly data of (a) net surface heat flux, (b) buoyancy frequency averaged over the upper $11 \mathrm{~m}$, (c) kinematic wind stress (crossand along-bank components combined), and (d) mixed layer depth for period II. Frequencies $>3$ cycles day $^{-1}$ are described by white noise and not shown. Vertical bars to the left give the $95 \%$ confidence limits. 
(a)

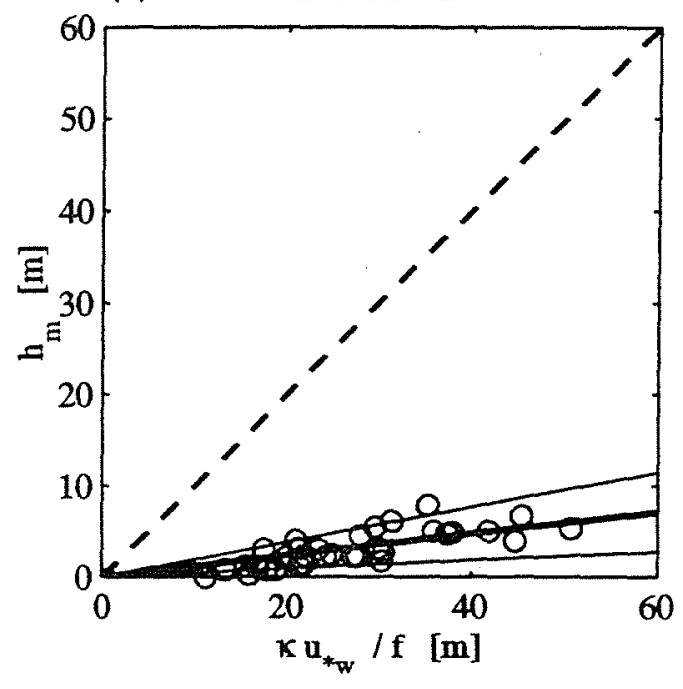

(b) Period IIB

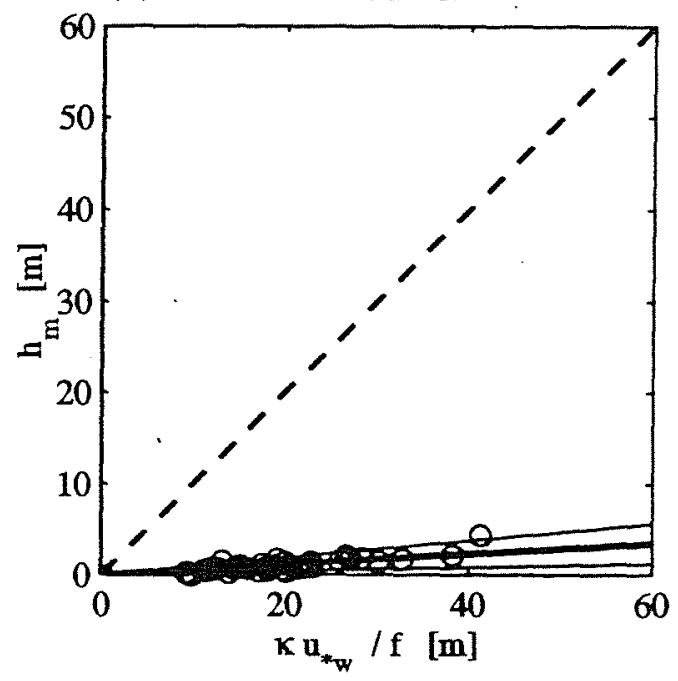

Fig.4.26: Daily averaged mixed layer depth $h_{m}$ versus daily averages of $\frac{\kappa u_{-} W}{f}$. The diffusive depth scale $\frac{\kappa u_{*} W}{f}$ was taken to represent the Ekman layer depth for unstratified conditions. The dashed line is a reference line that intersects the origin with slope 1.0. Also shown is the (heavy solid) linear regression between $h_{m}$ and $\frac{\kappa u_{+} \text {w }}{f}$ with slope (a) 0.12 and (b) 0.06 . Thin solid lines give the $95 \%$ confidence limits of the fits. Results are insensitive to whether or not a low-pass filter has been applied before the averaging. 
(a)

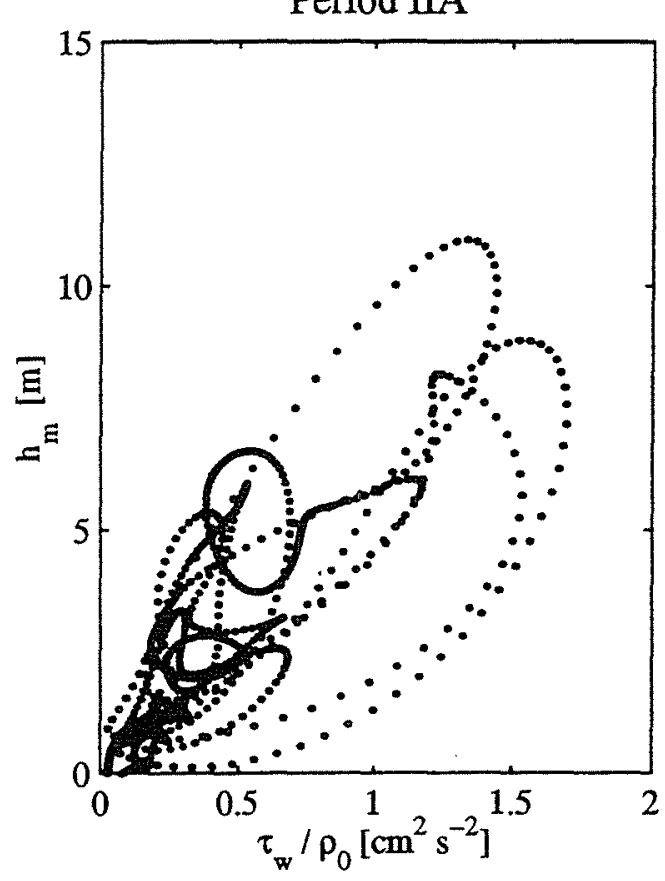

(b) Period IIB

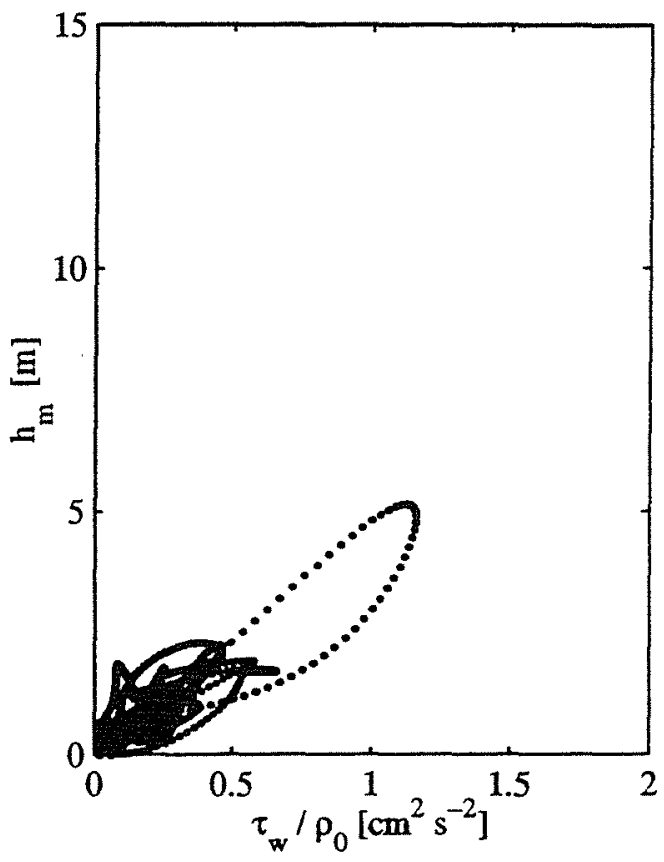

Fig.4.27: Hourly values of low-pass-filtered mixed layer depth versus kinematic wind stress magnitude for (a) period IIA and (b) period IIB. 


\section{Chapter 5}

\section{Conclusion}

This thesis presents results from observational and numerical investigation of the bottom and surface boundary layers on a shallow submarine bank, i.e., the southern flank of Georges Bank. Detailed analysis of the boundary layer structure was made possible through an extensive field program, which combined oceanographic and meteorological moored array data with bottom tripod measurements. Results of the analysis are aimed at improving the present understanding of boundary layer dynamics in the coastal ocean, and providing information valuable to interdisciplinary studies.

Although motivated by the physical processes on Georges Bank, the issues addressed in this thesis are of importance to coastal and estuarine regions in general. Since surface and bottom boundary layers are inherent features of the continental shelf circulation, accurate prediction of the involved flow, density, and turbulence fields by analytical, conceptual, and numerical models represents a topic of great interest to many physical and ecological studies. Obviously, this thesis cannot answer the open question how to parameterize turbulent mixing, nor can it provide a universal formula for the effects of boundary layer mixing on ocean physics. However, valuable insight is gained into tidal bottom boundary layer dynamics during various background conditions, the performance of several existing numerical models in predicting friction-induced turbulence, and processes affecting the vertical structure of the wind-driven flow. The following paragraphs will summarize the main results of the thesis and discuss their application to problems of local and global importance.

The investigation of the bottom boundary layer (BBL) in Chapters 2 and 3 clearly demonstrates that the BBL on the southern flank of Georges Bank is pre- 
dominantly tidally driven. As pointed out in previous studies, the structure and dynamics of the tidal BBL strongly depend on vertical stratification $N^{2}$, which acts to limit turbulent mixing in the pycnocline (e.g., Maas and van Haren, 1987; Soulsby, 1990; Souza and Simpson, 1996). Understanding of the BBL dynamics thus requires consideration of the time scales on which variation of $N^{2}$ occurs. These time scales range from hours to months depending on the physical processes involved. On time scales of hours, tidal advection of nearby density fronts, i.e., the tidal mixing and Shelf-Slope fronts, causes variability of $N^{2}$ at the period of the $M_{2}$ tide (12.42 hrs). Both observational and numerical results show semidiurnal variation of the density distribution can cause significant asymmetries in mixing intensity between on- and off-bank flow, which feed back on the velocity distribution during flood and ebb tides. These asymmetries are reinforced by the subtidal circulation, which adds to or subtracts from the tidal current shear depending on the phase of the tide. Since turbulent mixing processes are highly nonlinear, the effects of both tidal variation of $N^{2}$ and subtidal current shear have to be considered in conceptual and numerical models of the BBL.

On time scales of days to weeks, on- and off-bank motion of the Shelf-Slope front may cause significant changes in the density and $N^{2}$-distribution. The example presented in Chapter 3 demonstrates that the effects of a Shelf-Slope front intrusion on the water structure can be large and induce buoyancy frequencies that exceed those typical of summer. Observations and numerical results show the resulting impacts on BBL dynamics are significant. In particular, large buoyancy frequencies below mid-depths greatly limit the vertical extent of the turbulent BBL during part of the intrusion. The implication of such events on growth and survival rates of fish larvae, e.g., through reduction of the prey encounter rate due to decreasing turbulence in and above the density interface, is largely unknown and should be investigated in future studies.

Last, seasonal variation of the density distribution leads to changes in BBL dynamics through increasing surface heat flux and stratification as time progresses from winter to summer. The results are greater BBL thickness in winter than in summer, and the occurrence of internal tides during the stratified season. Data indicate that despite the observed seasonal changes in BBL thickness and mixing intensity, friction parameters such as the bottom friction velocity and quadratic drag coefficient do not display strong variation with season. This behavior shows 
growing vertical stratification does not cause significant reduction of the bottom stress, implying turbulence production at the lower boundary is roughly equally strong in summer and winter.

Comparison of observational data to results from one-dimensional turbulence closure models yields two important results. First, accurate prediction of the tidal currents and bottom stress in winter can be achieved with a relatively simple closure scheme that assumes a linear/constant distribution of the eddy viscosity $K$, where the $K$-profile near the bottom is in agreement with the logarithmic law of the wall (2LK model). Hence, modeling of the BBL during nearly homogeneous conditions does not require the application of advanced higher order schemes, in support of earlier conclusions by Davies (1991).

However, simplistic models such as the $2 \mathrm{LK}$ model cannot describe the turbulence field if the water column is stratified, i.e., if buoyancy forces act to suppress vertical mixing. For such conditions, higher order turbulence closure schemes such as the Mellor-Yamada level 2.5 (MY2.5) model represent a widely used approach to predict the flow physics. Comparison of the tidal BBL structure to results from the MY2.5 model yields the second main conclusion of the model-data comparison. The model performance is limited by the underestimation of the BBL thickness and the abrupt adjustment to a frictionless interior, a behavior that is particularly pronounced when stratification is large below mid-depth, but can also be observed at times when the pycnocline is weak and located above mid-depth. The most likely explanation for the model behavior is that the transition from molecular to turbulent mixing takes place abruptly near $R i=0.25$ in the MY2.5 model, while observation suggest mixing in the ocean can occur over a range of Richardson numbers including $0.25<R i<1.0$ (Polzin, 1996).

Since the MY2.5 model is widely used in coastal studies aimed at understanding the physical as well as coupled physical-biological processes, the results from the model-data comparison presented here give important insight into the model performance. For example, model predictions of fish survival rate on Georges Bank and in other regions depend on the ability of the mixing scheme to provide reliable estimates of turbulent dissipation and turbulent kinetic energy (e.g., Werner et al., 1996). Furthermore, second order terms such as nonlinear advection and vertical velocities are strongly affected by frictional processes. Hence, results from numerical circulation models that require detailed interpretation of the second order or 
mixing terms, e.g., upwelling or subduction near density fronts, divergence of the tidal Reynolds stress, or prey encounter rates of fish larvae, should be treated with caution.

Examination of the subtidal (temporal scales $>33 \mathrm{hrs}$ ) wind-driven circulation in Chapter 4 shows that the wind-induced currents fall into two categories. The first category represents the barotropic flow, i.e., the response to wind forcing that is in geostrophic balance with the sea surface slope generated by the presence of coastal boundaries and bottom topography. Observational results support earlier conclusions by Noble et al. (1985) stating that the along-bank flow on the southern flank of Georges Bank responds strongly to variation in along-bank wind stress, while the response to cross-bank wind forcing is weak. The focal point of this investigation is the second category of wind-induced flow, which constitutes the ageostrophic response to wind forcing (Ekman response). Opposed to the geostrophic response, the Ekman flow is not bound to follow the isobaths. Hence, the ageostrophic currents present an effective mechanism for cross-bank advection of water masses and marine organisms such as zooplankton and fish larvae. Chapter 4 presents a first step toward isolating the Ekman currents from the background flow and interpreting the results for conditions representative of winter and summer.

Observations show the vertical structure of the Ekman flow varies strongly with season, in agreement with earlier results from deep ocean studies (Schudlich and Price, 1998). While observations representative of winter form a well developed spiral that extends through most of the 76-m deep water column, the summer results are surface-trapped in the upper 10-19 $\mathrm{m}$ with flow directions almost at right angle to the wind.

Closer investigation shows the vertical structure of the Ekman currents cannot be explained satisfactory with analytical models assuming simple eddy viscosity parameterizations such as constant $K$ (Ekman, 1905) or a $K$-profile that increases linearly away from the surface (Madsen, 1977). Although details about the $K$-distribution cannot be inferred from the data presented in this thesis, observations give some indication about what physical processes determine the dynamics of the surface boundary layer. In winter, free convection presents an important mixing mechanism, which can have large impact on the wind-driven ageostrophic flow. Winter measurements show strong resemblance between the vertical structure of the estimated Ekman currents during three independent 1.2-2.6-day long wind 
events that were also events of significant surface heat loss. Although different in amplitude, all events display similar ratios of decay to rotation rates with depth, possibly indicating the depth-variation of $K$ was comparable. Similarities in $K$ distribution during the three events may be due to the homogenizing effects of free convection.

The limitation of the surface boundary layer thickness in summer is explained by weak wind forcing as well as strong vertical stratification. In agreement with theory, the lower boundary represented by the summer pycnocline results in a more slablike response of the wind-driven flow than for the winter case. Current amplitudes and veering of the velocity vector with depth depend on the details of the $\mathrm{N}^{2}$ distribution and its effects on turbulent mixing.

It is obvious from the summary given above that questions about how windinduced flow can be modeled relate back to the conclusion drawn in Chapters 2 and 3. Accurate prediction of the magnitude and vertical structure of the Ekman currents depends on the performance of the mixing scheme used to determine $K$. Development of reliable mixing parameterizations remains a topic of ongoing research, in particular with respect to the impacts of free convection and vertical stratification. Future progress in this question depends strongly on the availability of turbulence measurements taken during a variety of oceanographic conditions. Such measurements can then be used as a basis for deriving suitable quantitative expressions that describe turbulent mixing.

Finally, results from both the bottom and surface boundary layer analyses underline the importance of intermittent processes for the understanding and prediction of ecosystem changes on Georges Bank and in other regions. Intermittent intrusions of the Shelf-Slope front may significantly alter the water structure and mixing characteristics of the water column on time scales of days to weeks. The resulting effects on marine biology may be felt over times much longer than the event duration, e.g., due to starvation of larval/juvenile fish during the intrusion caused by reduced turbulence, and subsequent reduction of the fish population. Similarly, advective processes associated with individual strong storms can have severe impact on the abundance of zooplankton and fish throughout the year. For example, the wind event on February 5-8 caused a depth-averaged off-bank flow of $4 \mathrm{~cm} \mathrm{~s}^{-1}$, with flow velocities that reached about $13 \mathrm{~cm} \mathrm{~s}^{-1}$ in the upper $10 \mathrm{~m}$. Given the event duration of roughly 2.6 days, the off-bank distance covered by a water particle 
during this time is about $9 \mathrm{~km}$ in the depth-average, and $29 \mathrm{~km}$ near the surface. In comparison, the cross-bank separation distance between the tidal mixing and Shelf-Slope fronts is about $50 \mathrm{~km}$. Thus, wind events like the February storm advect water and organisms from the southern flank of Georges Bank into the region south of the Shelf Slope front, where they are lost to the Georges Bank circulation. Depending on when and how often such storms occur, the effects on zooplankton and fish populations may be dramatic. Clearly, the impacts of Shelf-Slope front intrusions and winter storms discussed above point at the necessity to incorporate such events into coupled bio-physical models of the Georges Bank area, as well as into models of other coastal environments.

\section{References}

Davies, A. M., On using turbulence energy models to develop spectral viscosity models. Continental Shelf Res., 11, 1,313-1,353, 1991.

Ekman, V. W., On the influence of the earth's rotation on ocean-currents. Ark. Mat., Astron. Bf Fys., 2, 55, 1905.

Maas, L. R. M., and J. J. M. van Haren, Observations on the vertical structure of tidal and inertial currents in the central North Sea. J. Marine Res., 45, 293-318, 1987.

Madsen, O.S., A realistic model of the wind-induced Ekman boundary layer. $J$. Phys. Oceanogr., 7, 248-255, 1977.

Noble, M., and B. Butman, and M. Wimbush, Wind-current coupling on the southern flank of Georges Bank: Variation with season and frequency. J. Phys. Oceanogr., 15, 604-620, 1985.

Polzin, K., Statistics of the Richardson Number: Mixing Models and Finestructure. J. Physical Oceanogr.,26, 1409-1425, 1996.

Schudlich, R.R., and James F. Price, Observations of Seasonal Variation in the Ekman Layer. J. Phys. Oceanogr., 28 , 1187-1204, 1998.

Soulsby, R. L., Tidal-current boundary layers. The Sea, 9, 523-566, 1990. 
Souza, A. J., and J. H. Simpson, The modification of tidal ellipses by stratification in the Rhine ROFI. Continental Shelf Res., 16, 997-1007, 1996.

Werner, F.E., R.I. Perry, R.G. Lough, and C.E. Naimie, Trophodynamics and advective influences on Georges Bank larval cod and haddock. Deep Sea Res., Part II, 43, 1793-1822, 1996. 